\title{
Sequential Photocatalytic Reactions for the Diastereoselective Synthesis of Cyclobutane Scaffolds
}

Mark J. Deeprose, ${ }^{\dagger}$ Martin Lowe, ${ }^{\ddagger}$ Adam Noble, ${ }^{*}$ Kevin I. Booker-Milburn, ${ }^{\dagger *}$ and Varinder K. Aggarwal ${ }^{\dagger *}$

†School of Chemistry, University of Bristol, Cantock’s Close, Bristol, BS8 1TS, UK

¥Medicinal Chemistry Department, UCB, 216 Bath Road, Slough, SL1 3WE, UK

*E-mail: a.noble@bristol.ac.uk, k.booker-milburn@bristol.ac.uk, v.aggarwal@bristol.ac.uk 


\section{Table of Contents}

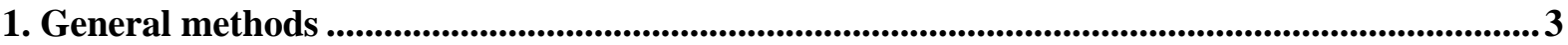

1.1. Solvents, Reagents, Glassware and Reaction Setup............................................................... 3

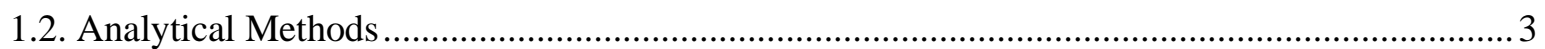

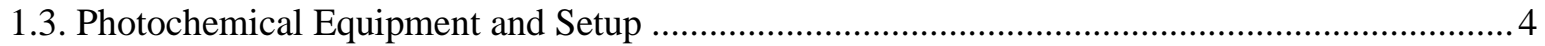

2. General Procedures ..................................................................................................................................................5

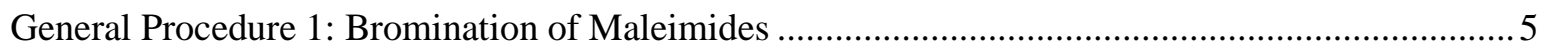

General Procedure 2: Chlorination of Bromomaleimides..............................................................

General Procedure 3: [2+2] Photocycloadditions of Chloromaleimides ........................................ 6

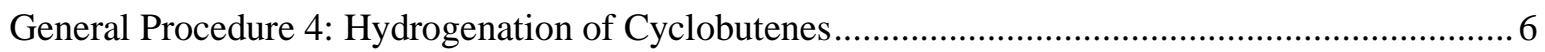

General Procedure 5: Photoredox-Catalysed Dechlorinative Functionalisations of Cyclobutanes .... 7

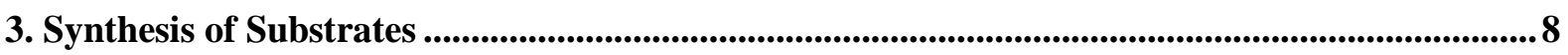

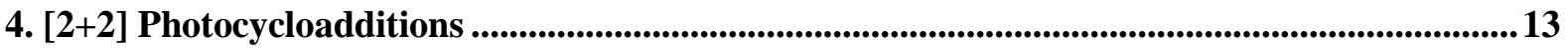

4.1. Comparison of the Selectivity for Reactions of Alkenes and Alkynes .................................... 13

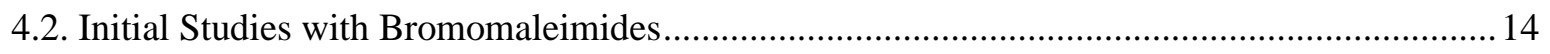

4.3. Effect of ITX on [2+2] Photocycloaddition Reactions ….................................................... 15

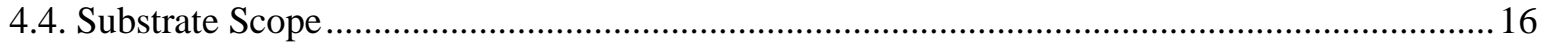

5. Photoredox-Catalysed Dechlorinative Functionalisations .........................................................25

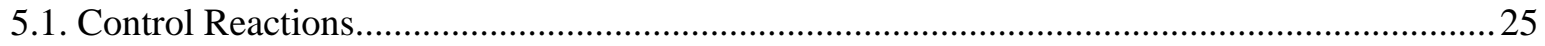

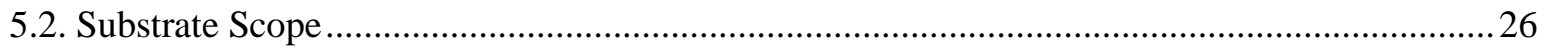

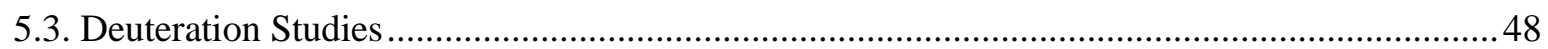

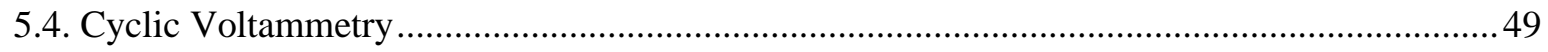

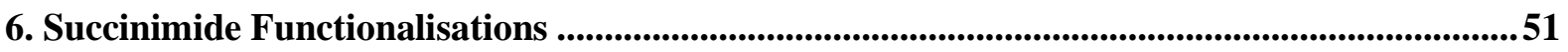

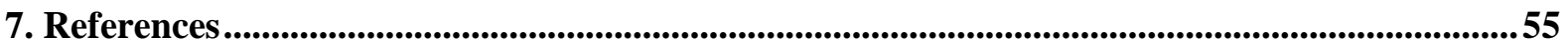

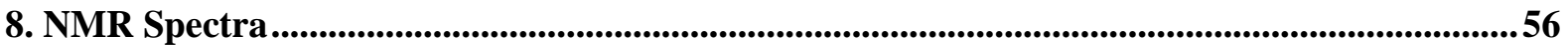




\section{General methods}

\subsection{Solvents, Reagents, Glassware and Reaction Setup}

All reactions, unless otherwise specified, were conducted under normal atmospheric conditions with no need for drying glassware or alterations to commercially sourced chemicals. Air- and moisture-sensitive liquids and solutions were transferred via syringe into the reaction vessels through rubber septa. Heating of reactions, where required, was achieved by placing the reaction vessel in an oil bath at the specified temperature. Unless otherwise specified, all reagents were purchased at highest commercial quality and used as received. Non-anhydrous solvents were purchased (unless specified) at the highest commercial quality and used as received. Acetone and diethyl ether were purchased from Sigma. Acetonitrile $(\mathrm{MeCN})$, dichloromethane (DCM) and tetrahydrofuran (THF) were dried on an Anhydrous Engineering alumina column drying system.

\subsection{Analytical Methods}

Chromatography: Flash column chromatography was carried out using Sigma-Aldrich silica gel (60 $\AA$ A, 230-400 mesh, 40-63 $\mu \mathrm{m}$ ) flash purification system. Reactions were followed by thin-layer chromatography (TLC), where practical, using aluminium-backed Merck Kieselgel 60 F254 fluorescent treated silica gel plates, which were visualised under UV light or by staining with aqueous basic $\mathrm{KMnO}_{4}$, acidic $p$-anisaldehyde solution in ethanol, or phosphomolybdic acid solution in ethanol.

IR: IR spectra were recorded on neat compounds using a Perkin Elmer (Spectrum One) FT-IR spectrometer (ATR sampling accessory). Selected absorbances $\left(v_{\max }\right.$, expressed in $\left.\mathrm{cm}^{-1}\right)$ are reported.

${ }^{1}$ H NMR: Spectra were recorded on Jeol ECS (400 MHz), Jeol ECZ (400 MHz or Bruker Avance (400 $\mathrm{MHz}$ or $500 \mathrm{MHz}$ ) instruments. Chemical shifts ( $\delta$ ) are quoted in parts per million (ppm) and referenced to the appropriate NMR solvent peak(s). Coupling constants $(J)$ are given in Hertz (Hz) and refer to apparent multiplicities $(\mathrm{s}=$ singlet, $\mathrm{br} . \mathrm{s}=$ broad singlet, $\mathrm{d}=$ doublet, $\mathrm{t}=$ triplet, $\mathrm{q}=$ quartet, $\mathrm{p}=$ pentet, $\mathrm{m}=$ multiplet, $\mathrm{dd}=$ doublet of doublets, etc.). The ${ }^{1} \mathrm{H}$ NMR spectra are reported as follows: chemical shift (multiplicity, coupling constants, number of protons).

${ }^{13}$ C NMR: Spectra were recorded on a Jeol ECS (100 MHz), Jeol ECZ (100 MHz or Bruker Avance $(100 \mathrm{MHz}$ or $125 \mathrm{MHz})$ instruments. Chemical shifts $(\delta)$ are quoted in parts per million $(\mathrm{ppm})$ and referenced to the appropriate NMR solvent peak(s).

HRMS: High resolution mass spectrometry was performed on a Bruker Daltronics MicroTOF II (ESI), Thermo Scientific Orbitrap (ESI, APCI) or Thermo Scientific QExactive (EI). Only molecular ion peaks $\left([\mathrm{M}+\mathrm{H}]^{+}\right.$or $[\mathrm{M}+\mathrm{Na}]^{+}$for ESI and $\mathrm{APCI} ; \mathrm{M}^{+}$for $\left.\mathrm{EI}\right)$ are reported.

LRMS: Low resolution mass spectrometry was performed using an Agilent Technologies 7890B GC system using electron ionisation (EI). Only major peaks are reported. 
Mp: Melting points were recorded in degrees Celsius $\left({ }^{\circ} \mathrm{C}\right)$, using a Stuart SMP30 melting point apparatus and are reported uncorrected.

\subsection{Photochemical Equipment and Setup}

The UV lamps used were $125 \mathrm{~W}$ medium pressure Hg lamp $\left(\lambda_{\max }=366 \mathrm{~nm}\right)$. The UV lamp with Pyrex ${ }^{\circledR}$ cooling jacket was immersed in the reaction solution and with a positive pressure of nitrogen sparging through the solution and out through a paraffin oil bubbler (Figure S1).

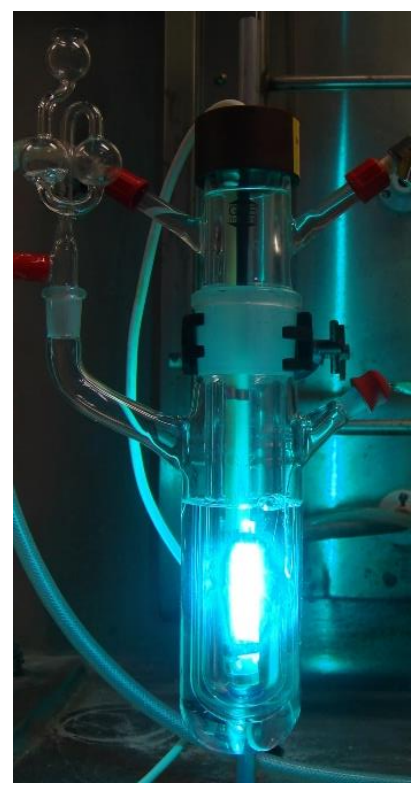

Figure S1. [2+2] Photocycloaddition immersion well setup.

The blue LEDs used were $36 \mathrm{~W}$ blue chip-on-board (COB) LEDs manufactured by Citizen Electronics (CLU048-1212-B455, $\lambda_{\max }=455 \mathrm{~nm}$ ). All photocatalysed dehalogenation reactions were carried out at room temperature (r.t.) with fan-assisted cooling to maintain a temperature of $30-40{ }^{\circ} \mathrm{C}$. The reactions were run in $50 \mathrm{~mL}$ round bottomed flasks positioned $3 \mathrm{~cm}$ above a single $36 \mathrm{~W}$ COB LED with a fan positioned next to the reaction (Figure S2).

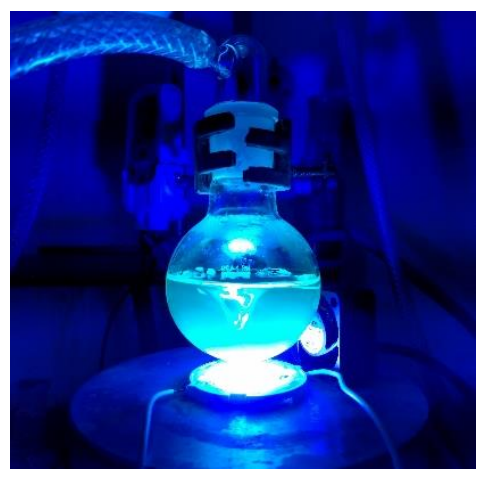

Figure S2. Visible-light photocatalysed dehalogenation reaction setup. 


\section{General Procedures}

\section{General Procedure 1: Bromination of Maleimides}

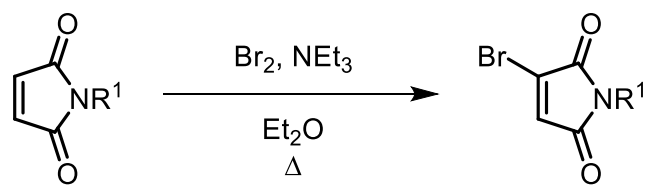

To a round bottomed flask equipped with a stirrer bar, a solution of specified maleimide (100 mol\%) and diethyl ether $(0.7 \mathrm{M})$ was made. To this, bromine $(110 \mathrm{~mol} \%)$ was added over 5 minutes at r.t. and left to stir at reflux for 2 hours. On completion of bromination, the solution should turn lighter in colour. To this, triethylamine $(110 \mathrm{~mol} \%)$ was added over 5 minutes at $0{ }^{\circ} \mathrm{C}$. The solution was left to stir for 18 hours. The solids were removed by filtration through celite, and the filtrate was concentrated in vacuo to give the crude product as an orange oil. The crude product was diluted with EtOAc (1.5 $\mathrm{mL} / \mathrm{mmol})$, washed with water $(1 \mathrm{~mL} / \mathrm{mmol})$ and brine $(0.5 \mathrm{~mL} / \mathrm{mmol})$ and dried over $\mathrm{MgSO}_{4}$. The solvent was removed in vacuo and the resulting oil was triturated in petroleum ether (40/60), and the supernatant carefully decanted off. This step was repeated twice more. The solids obtained were collected by filtration through a glass frit and washed with ice cold methanol to give the bromomaleimide product.

\section{General Procedure 2: Chlorination of Bromomaleimides}

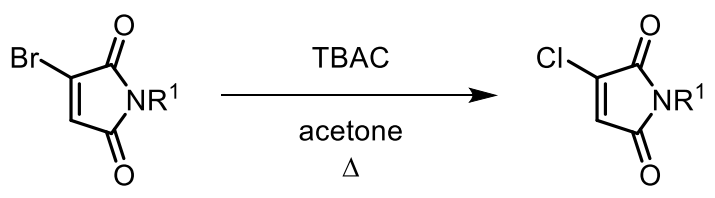

To a round bottomed flask equipped with stirrer bar, a solution of bromomaleimide (100 mol\%) and tetrabutylammonium chloride (TBAC, $110-300 \mathrm{~mol} \%)$ was made in acetone $(0.3 \mathrm{M})$ and heated to reflux for 1-4 hours. The solvent was removed in vacuo and the crude material was diluted in EtOAc $(1 \mathrm{~mL} / \mathrm{mmol})$ and washed with water $(1 \mathrm{~mL} / \mathrm{mmol})$ and saturated aqueous $\mathrm{NH}_{4} \mathrm{Cl}(1 \mathrm{~mL} / \mathrm{mmol})$. The organic layer was dried over $\mathrm{MgSO}_{4}$, and the solvent removed to give the crude product, which was used without further purification. The chloromaleimides were found to be stable to column chromatography on silica gel, however, upon concentration under vacuum they were found to be unstable to polymerisation. 


\section{General Procedure 3: [2+2] Photocycloadditions of Chloromaleimides}

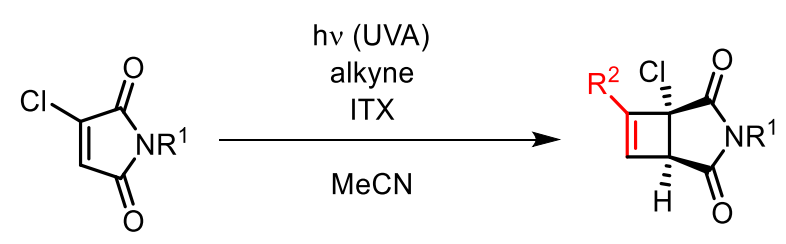

Batch: In a UV reaction vessel, a solution of the chloromaleimide (100 mol\%) and degassed $\mathrm{MeCN}$ $(0.1 \mathrm{M})$ was made. The alkyne substrate (150-300 mol\%) and 2-isopropylthioxanthone (ITX, $1 \mathrm{~mol} \%)$ were then added. The resulting mixture was exposed to UV light while being agitated by bubbling nitrogen at r.t. for the specified amount of time. The solution was concentrated in vacuo to give the crude cyclobutene product.

Syringe Pump Addition: In a UV reaction vessel, a solution of the alkyne substrate (300 mol\%) and ITX $(1 \mathrm{~mol} \%)$ in degassed MeCN $(0.1 \mathrm{M})$ was made. This was exposed to UV light while being agitated by bubbling nitrogen at r.t. A solution of chloromaleimide (100 mol\%) in degassed $\mathrm{MeCN}(1 \mathrm{~mL} / \mathrm{mmol})$ was added dropwise over the specified time to the substrate solution. Irradiation was continued until TLC indicated the reaction was complete. The solution was concentrated in vacuo to give the crude cyclobutene product.

\section{General Procedure 4: Hydrogenation of Cyclobutenes}

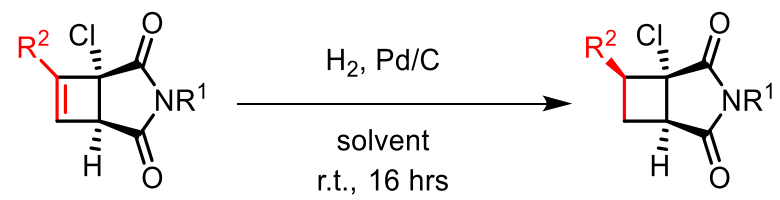

In a $250 \mathrm{~mL}$ round bottomed flask, a mixture of cyclobutene (100 mol\%), 5\% Pd/C (5\% w/w) and methanol $(0.1 \mathrm{M})$ was made. This was sparged for 15 minutes with $\mathrm{H}_{2}$ and left under a $\mathrm{H}_{2}$ atmosphere (balloon) at r.t. for 16 hours. The $\mathrm{Pd} / \mathrm{C}$ was removed by vacuum filtration over celite, and the filtrate was concentrated in vacuo. Purification by column chromatography gave the chlorocyclobutane product. 
General Procedure 5: Photoredox-Catalysed Dechlorinative Functionalisations of Cyclobutanes
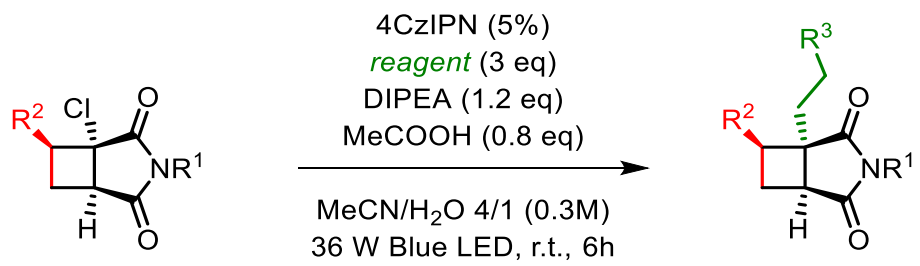

In a $50 \mathrm{~mL}$ round bottomed flask, a solution of chlorocyclobutane (0.65 mmol, $100 \mathrm{~mol} \%)$, diisopropylethylamine (DIPEA, $120 \mathrm{~mol} \%$ ), 4CzIPN (5 mol\%), acetic acid (80 mol\%), and the specified reagent $(300 \mathrm{~mol} \%$ ) in $\mathrm{MeCN} /$ water (4/1, $2.0 \mathrm{~mL}$ ) was sparged with nitrogen for 10 minutes. The solution was exposed to a $36 \mathrm{~W}$ Blue LED $(455 \mathrm{~nm})$ for the specified time. On completion, water $(20 \mathrm{~mL})$ was added, and the mixture extracted with ethyl acetate $(2 \times 30 \mathrm{~mL})$. The combined extracts were dried over $\mathrm{MgSO}_{4}$, and concentrated in vacuo. Purification by column chromatography gave the functionalised cyclobutane product. 


\section{Synthesis of Substrates}

\section{1-Benzyl-3-bromo-1H-pyrrole-2,5-dione (1a)}

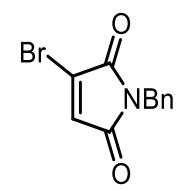

Prepared according to General Procedure 1, using $N$-benzyl maleimide $(107 \mathrm{mmol})$ and diethyl ether (156 mL). Purification by washing with cold methanol gave the title compound ( $25.8 \mathrm{~g}, 90 \%)$ as a beige solid.

${ }^{1} \mathbf{H}$ NMR $\left(400 \mathrm{MHz}, \mathrm{CDCl}_{3}\right) \delta_{\mathrm{H}} 7.39-7.27(\mathrm{~m}, 5 \mathrm{H}), 6.87(\mathrm{~s}, 1 \mathrm{H}), 4.71(\mathrm{~s}, 2 \mathrm{H})$. See spectrum.

${ }^{13} \mathrm{C}$ NMR $\left(100 \mathrm{MHz}, \mathrm{CDCl}_{3}\right) \delta_{\mathrm{C}} 168.2,165.0,135.6,131.9,131.5,128.8,128.6,128.1,42.4 . \underline{\text { See }}$ spectrum.

Mp (petroleum ether): $47-50{ }^{\circ} \mathrm{C}$.

All recorded data match literature values. ${ }^{1}$

\section{3-Bromo-1-methyl-1H-pyrrole-2,5-dione (1b)}

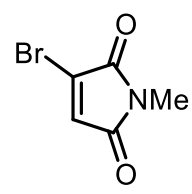

Prepared according to General Procedure 1, using $N$-methylmaleimide (2.00 g, $18.0 \mathrm{mmol}$ ) and diethyl ether $(25 \mathrm{~mL})$. The brown crystals were washed with pentane to give the title compound $(2.77 \mathrm{~g}, 82 \%)$ as pale brown platelets.

${ }^{1} \mathbf{H}$ NMR $\left(500 \mathrm{MHz}, \mathrm{CDCl}_{3}\right) \delta_{\mathrm{H}} 6.90(\mathrm{~s}, 1 \mathrm{H}), 3.10(\mathrm{~s}, 3 \mathrm{H})$. $\underline{\text { See spectrum. }}$

${ }^{13}$ C NMR $\left(125 \mathrm{MHz}, \mathrm{CDCl}_{3}\right) \delta_{\mathrm{C}} 168.6,165.4,131.9,131.4,24.6$. See spectrum.

Mp (petroleum ether): $102-105^{\circ} \mathrm{C}$.

All recorded data match literature values. ${ }^{2}$ 


\section{3-Bromo-1H-pyrrole-2,5-dione (1c)}

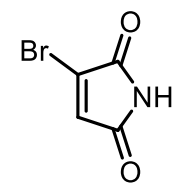

A solution of $1 H$-maleimide (3.9 g, $40 \mathrm{mmol}), \mathrm{Br}_{2}(2.5 \mathrm{~mL}, 50 \mathrm{mmol})$ and $\mathrm{DCM}(60 \mathrm{~mL})$ was stirred at reflux. On completion of bromination, the solvent and excess bromine were removed in vacuo and the crude material was dissolved in THF $(80 \mathrm{~mL})$. Triethylamine $(6 \mathrm{~mL}, 40 \mathrm{mmol})$ was added slowly over 5 mins at $0{ }^{\circ} \mathrm{C}$. The solution was allowed to warm to r.t. and stirred for 3 hours. Subsequent work up, following the procedure described in general procedure 1 , gave the title compound $(5.60 \mathrm{~g}, 80 \%)$ as a colourless solid.

${ }^{1} \mathbf{H}$ NMR $\left(500 \mathrm{MHz}, \mathrm{CDCl}_{3}\right) \delta_{\mathrm{H}} 7.49(\mathrm{~s}, 1 \mathrm{H}), 6.91(\mathrm{~s}, 1 \mathrm{H})$. See spectrum.

${ }^{13}$ C NMR $\left(125 \mathrm{MHz}, \mathrm{CDCl}_{3}\right) \delta_{\mathrm{C}} 167.7,164.7,132.9,132.2$. See spectrum.

Mp (Petroleum ether): $157-159^{\circ} \mathrm{C}$.

All recorded data match literature values. ${ }^{3}$

\section{1-Benzyl-3-chloro-1H-pyrrole-2,5-dione (2a)}

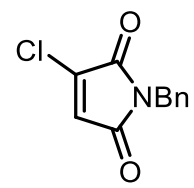

Prepared according to general procedure 2, using 1-benzyl-3-bromomaleimide (5.00 g, $18.8 \mathrm{mmol})$, tetrabutylammonium chloride $(5.75 \mathrm{~g}, 20.7 \mathrm{mmol})$ and acetone $(56 \mathrm{~mL})$, with a reaction time of 1 hour. The crude material was used without further purification.

The yield was determined to be $90 \%$ by ${ }^{1} \mathrm{H}$ NMR using 1,3,5-trimethoxy benzene as an internal standard.

${ }^{1} \mathrm{H}$ NMR $\left(500 \mathrm{MHz}, \mathrm{CDCl}_{3}\right) \delta_{\mathrm{H}} 7.20-7.05(\mathrm{~m}, 5 \mathrm{H}), 6.50(\mathrm{~s}, 1 \mathrm{H}), 4.51(\mathrm{~s}, 2 \mathrm{H})$. See spectrum.

${ }^{13} \mathrm{C}$ NMR $\left(125 \mathrm{MHz}, \mathrm{CDCl}_{3}\right) \delta_{\mathrm{C}} 167.3,164.5,140.8,135.5,128.6,128.3,128.0,126.8,42.1 . \underline{\text { See }}$ spectrum.

LRMS m/z: [M] calcd for $\mathrm{C}_{11} \mathrm{H}_{8} \mathrm{ClNO}_{2} 221.0$, found 221.0. 


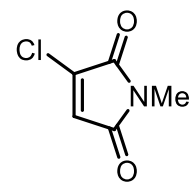

Prepared according to general procedure 2, using 1-methyl-3-bromomaleimide (1.30 g, $6.80 \mathrm{mmol})$, tetrabutylammonium chloride $(2.78 \mathrm{~g}, 10.0 \mathrm{mmol})$ and acetone $(20 \mathrm{~mL})$, with a reaction time of 2 hours. The crude material was used without further purification.

The yield was determined to be $70 \%$ by ${ }^{1} \mathrm{H}$ NMR using 1,3,5-trimethoxy benzene as an internal standard.

${ }^{1} \mathrm{H}$ NMR $\left(500 \mathrm{MHz}, \mathrm{CDCl}_{3}\right) \delta_{\mathrm{H}} 6.39(\mathrm{~s}, 1 \mathrm{H}), 2.72(\mathrm{~s}, 3 \mathrm{H}) . \underline{\text { See spectrum. }}$

${ }^{13}$ C NMR $\left(125 \mathrm{MHz}, \mathrm{CDCl}_{3}\right) \delta_{\mathrm{C}} 167.5,164.8,140.6,126.8,24.2$. See spectrum.

LRMS m/z: [M] calcd for $\mathrm{C}_{5} \mathrm{H}_{4} \mathrm{ClNO}_{2}$ 145.0, found 145.0.

\section{1-Benzyl-3-chloro-1H-pyrrole-2,5-dione (2c)}

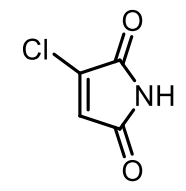

Prepared according to general procedure 2, using 1H-3-bromomaleimide (1.32 g, $7.50 \mathrm{mmol})$, tetrabutylammonium chloride $(6.25 \mathrm{~g}, 22.5 \mathrm{mmol})$ and acetone $(22 \mathrm{~mL})$, with a reaction time of 4 hours. The crude material was used without further purification.

The yield was determined to be $81 \%$ by ${ }^{1} \mathrm{H}$ NMR using $1,3,5$-trimethoxy benzene as an internal standard.

${ }^{1} \mathbf{H}$ NMR $\left(500 \mathrm{MHz}, \mathrm{CDCl}_{3}\right) \delta_{\mathrm{H}} 11.86(\mathrm{~s}, 1 \mathrm{H}), 6.18(\mathrm{~s}, 1 \mathrm{H})$. See spectrum.

${ }^{13}$ C NMR $\left(125 \mathrm{MHz}, \mathrm{CDCl}_{3}\right) \delta_{\mathrm{C}} 168.7,165.7,140.5,127.1$. See spectrum.

LRMS m/z: [M] calcd for $\mathrm{C}_{4} \mathrm{H}_{2} \mathrm{ClNO}_{2}$ 130.9, found 130.9 . 


\section{3-(But-3-en-1-yloxy)-4-chloro-1-methyl-1H-pyrrole-2,5-dione (S1)}<smiles>C=CCCOC1=C(Cl)C(=O)N(C)C1=O</smiles>

To a flame dried two-necked round bottomed flask, 2,3-dichloro- $N$-methylmaleimide (150 mg, 0.833 $\mathrm{mmol})$ and dry dioxane $(8.4 \mathrm{~mL})$ were added and stirred under a nitrogen atmosphere. In a separate flask, 3-buten-1-ol (145 $\mu \mathrm{L}, 1.68 \mathrm{mmol})$ was added dropwise over 5 minutes to a suspension of sodium hydride (60\% suspension in mineral oil, $50.4 \mathrm{mg}, 1.26 \mathrm{mmol})$ in dry dioxane (13 mL) and r.t., and the mixture was stirred at r.t. for a further 15 minutes. The resulting alkoxide solution was added dropwise over 5 minutes to the solution of maleimide and the reaction stirred at r.t. for 18 hours. Saturated aqueous ammonium chloride $(40 \mathrm{~mL})$ was added, and the mixture was extracted with ethyl acetate $(2 \times$ $20 \mathrm{~mL})$. The combined extracts were washed with water $(40 \mathrm{~mL})$, then brine $(40 \mathrm{~mL})$, and concentrated in vacuo. Purification by column chromatography (19/1 to 9/1 petroleum ether/ethyl acetate:) to give the title compound (112 $\mathrm{mg}, 62 \%)$ as a pale-yellow oil.

${ }^{1} \mathbf{H}$ NMR $\left(400 \mathrm{MHz}, \mathrm{CDCl}_{3}\right) \delta_{\mathrm{H}} 5.88-5.73(\mathrm{~m}, 1 \mathrm{H}), 5.23-5.08(\mathrm{~m}, 2 \mathrm{H}), 4.71-4.61(\mathrm{~m}, 2 \mathrm{H}), 3.02(\mathrm{~s}$, $3 \mathrm{H}), 2.62-2.49(\mathrm{~m}, 2 \mathrm{H})$. See spectrum.

${ }^{13} \mathbf{C}$ NMR $\left(100 \mathrm{MHz}, \mathrm{CDCl}_{3}\right) \delta_{\mathrm{C}} 165.7,164.2,150.1,132.4,118.5,103.8,71.6,34.0,24.2$. See spectrum. IR $v_{\max }: 2954,1713,1651,1440,1285,1048,922,739 \mathrm{~cm}^{-1}$.

HRMS (ESI) m/z: [(M+Na) $)^{+}$calcd for $\mathrm{C}_{9} \mathrm{H}_{10} \mathrm{ClNO}_{3} 238.0241$, found 238.0248.

\section{tert-Butyl 4-methylenepiperidine-1-carboxylate (S2)}

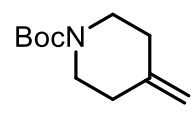

In a flame dried two-neck round bottomed flask, a solution of dry THF (100 mL) and methyltriphenylphosphonium bromide $(10.80 \mathrm{~g}, 30.00 \mathrm{mmol})$ was made under a nitrogen atmosphere. To this, $n$-butyllithium $(2.5 \mathrm{M}$ in hexanes, $12 \mathrm{~mL}, 30 \mathrm{mmol})$ was added dropwise at $-78{ }^{\circ} \mathrm{C}$ over 5 minutes and left to stir for 1 hour. To this solution, $N$-boc-piperidone was added before being allowed to warm to r.t. and subsequently stirred for 16 hours. The solvent was removed in vacuo and the residue was purified by column chromatography (70/30 petroleum ether/ethyl acetate) to give the title compound $(1.86 \mathrm{~g}, 31 \%)$ as a colourless oil.

${ }^{1} \mathbf{H}$ NMR $\left(400 \mathrm{MHz}, \mathrm{CDCl}_{3}\right) \delta_{\mathrm{H}} 4.76(\mathrm{q}, J=1.1 \mathrm{~Hz}, 2 \mathrm{H}), 3.48-3.40(\mathrm{~m}, 4 \mathrm{H}), 2.20(\mathrm{ddt}, J=6.6,4.5$, $1.0 \mathrm{~Hz}, 4 \mathrm{H}), 1.49(\mathrm{~s}, 9 \mathrm{H})$. 
${ }^{13} \mathrm{C}$ NMR $\left(100 \mathrm{MHz}, \mathrm{CDCl}_{3}\right) \delta_{\mathrm{C}} 154.8,145.5,109.0,79.5,45.4,34.5,28.5$.

All recorded data match literature values. ${ }^{4}$

\section{2-(1-Phenylvinyl)naphthalene (S3)}<smiles>C=C(c1ccccc1)c1ccc2ccccc2c1</smiles>

To a flame-dried two-necked round bottomed flask, a solution of phenyl magnesium bromide in THF (4.7 $\mathrm{mL}, 13.2 \mathrm{mmol})$ was added to a solution of $\beta$-napthyl-methyl ketone $(1.00 \mathrm{~g}, 6.00 \mathrm{mmol})$ and THF $(10 \mathrm{~mL})$ under a nitrogen atmosphere and stirred at r.t. for 3 hours. The solution was quenched with saturated aqueous $\mathrm{NH}_{4} \mathrm{Cl}(10 \mathrm{~mL})$ and the THF was removed in vacuo. The remaining crude solution was extracted with ethyl acetate $(2 \times 10 \mathrm{~mL})$ and the combined organic extracts were dried over $\mathrm{MgSO}_{4}$ and filtered. To the filtrate was added $p$-toluenesulfonic acid (23 $\mathrm{mg}, 0.12 \mathrm{mmol})$, and the mixture was stirred at r.t. for 10 hours. The solvent was removed in vacuo and the residue was purified by column chromatography (100\% petroleum ether) to give the title compound ( $685 \mathrm{mg}, 50 \%)$ as a white solid.

${ }^{1} \mathbf{H}$ NMR $\left(400 \mathrm{MHz}, \mathrm{CDCl}_{3}\right) \delta_{\mathrm{H}} 7.91-7.80(\mathrm{~m}, 4 \mathrm{H}), 7.55-7.47(\mathrm{~m}, 3 \mathrm{H}), 7.46-7.36(\mathrm{~m}, 5 \mathrm{H}), 5.63(\mathrm{~d}$, $J=1.2 \mathrm{~Hz}, 1 \mathrm{H}), 5.59(\mathrm{~d}, J=1.2 \mathrm{~Hz}, 1 \mathrm{H})$.

${ }^{13} \mathrm{C}$ NMR $\left(100 \mathrm{MHz}, \mathrm{CDCl}_{3}\right) \delta_{\mathrm{C}} 150.1,141.5,138.9,133.3,133.0,130.1,128.4,128.4,128.3,128.2$, $127.8,127.7,127.6,127.3,127.2,126.4,126.2,126.0,114.8$.

All recorded data match literature values. ${ }^{5}$

\section{Trimethyl((1-phenylvinyl)oxy)silane (S4)}<smiles>C=C(O[As])c1ccccc1</smiles>

To a flame dried two-necked round bottomed flask, acetophenone (1.00 mL, $8.57 \mathrm{mmol})$, NaI (124 mol\%), triethylamine (124 mol\%) were dissolved in $\mathrm{MeCN}(8.6 \mathrm{~mL})$ under a nitrogen atmosphere at r.t. To this, TMSCl (124 mol\%) was added dropwise over 5 minutes. A white precipitate was formed on addition. The mixture was left to stir at r.t. for 1 hour. The mixture was cooled to $0{ }^{\circ} \mathrm{C}$ and cold pentane $(20 \mathrm{~mL})$ and cold water $(20 \mathrm{~mL})$ were added. The aqueous layer was extracted with pentane (2 $\times 20 \mathrm{~mL})$ and the organic layers were washed with saturated aqueous $\mathrm{NH}_{4} \mathrm{Cl}(2 \times 10 \mathrm{~mL})$. The combined organic layers were dried over $\mathrm{MgSO}_{4}$, and the solvent was removed in vacuo to give the title compound $(1.40 \mathrm{~g}, 85 \%)$ as a colourless oil, which was used without further purification. ${ }^{6}$ 


\section{4. $[2+2]$ Photocycloadditions}

\subsection{Comparison of the Selectivity for Reactions of Alkenes and Alkynes}

The reaction of chloromaleimide 2a with allyl alcohol (Scheme S1) demonstrates a major issue with crossed [2+2] photocycloadditions with two unsymmetrical alkenes, which is the low regioselectivity (2.5:1) and moderate diastereoselectivity $(6: 1-7: 1)$. However, if alkynes are used, the number of possible products is reduced from four to two, and a higher regioselectivity (5:1) is observed.<smiles>O=C1C=C(Cl)C(=O)[NH2+]1</smiles>

2a

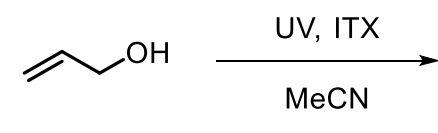

$77 \%$<smiles>O=C1[C@H](CO)C[C@]2(Cl)C(=O)N(Cc3ccccc3)C(=O)[C@H]12</smiles>

3:17 r.r.<smiles>O=C1[C@H](CO)C(=O)[C@@]2(Cl)C[C@H](Cc3ccccc3)[C@H]12</smiles>

$1: 7$ r.r.

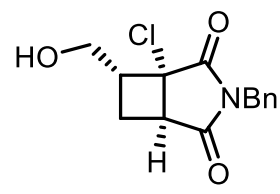<smiles></smiles>

2a<smiles>C#CCO</smiles>

$\underset{\mathrm{MeCN}}{\stackrel{\text { UV, ITX }}{\longrightarrow}}$

$75 \%$<smiles>O=C1[C@H]2C=C(CO)[C@]2(Cl)C(=O)N1Cc1ccccc1</smiles>

5:1 r.r.<smiles>O=C1[C@H]2C(CO)=C[C@]2(Cl)C(=O)N1Cc1ccccc1</smiles>$$
\text { O }
$$

Scheme S1. Comparison of the selectivity in [2+2] photocycloaddition of alkenes and alkynes. 


\subsection{Initial Studies with Bromomaleimides}

Bromomaleimide 1a was employed in [2+2] photocycloaddition with 1-hexyne to give the cyclobutene adduct in excellent regioselectivity (Scheme S2). Unfortunately, the yield for the cycloaddition was moderate $(44 \%)$ and the reaction proved difficult to scale up due to the poor stability of the bromomaleimide under the UV conditions. Furthermore, attempts to access the cyclobutane ring by hydrogenation of the cyclobutene over $\mathrm{Pd} / \mathrm{C}$ gave a 1:1 mixture of bromocyclobutane and hydrodebrominated cyclobutane. Therefore, this substrate was not explored further.<smiles>O=C1C=C(Br)C(=O)N1Br</smiles>

1a<smiles>C#C[13CH3]</smiles>

(3.0 equiv)
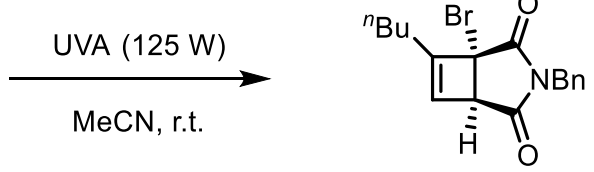

$44 \%$ 19:1 r.r.
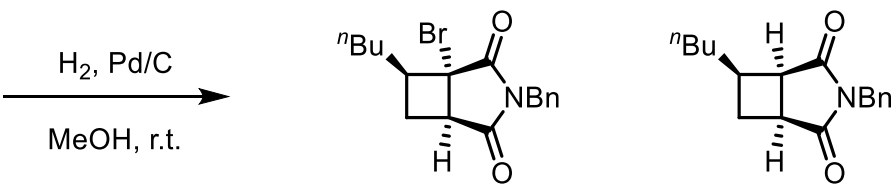

$1: 1$

Scheme S2. Bromomaleimide [2+2] photocycloaddtion and protodehalogenation issues. 


\subsection{Effect of ITX on [2+2] Photocycloaddition Reactions}

Initial studies into the synthesis of chlorocyclobutenes by direct photoexcitation of chloromaleimide $\mathbf{2 a}$ gave good yields of the [2+2] cycloadduct, but low productivity (Table S1, entry 1). Therefore, different photosensitisers were screened (entries 2-3). It was found that both 3-MeOTX and ITX were good candidates for energy transfer to chloromaleimide at $1 \mathrm{~mol} \%$ loading. Using these conditions, large scale production $(23 \mathrm{mmol} / \mathrm{h})$ of the cyclobutene can be achieved.

Table S1. [2+2] photocycloaddition optimisations

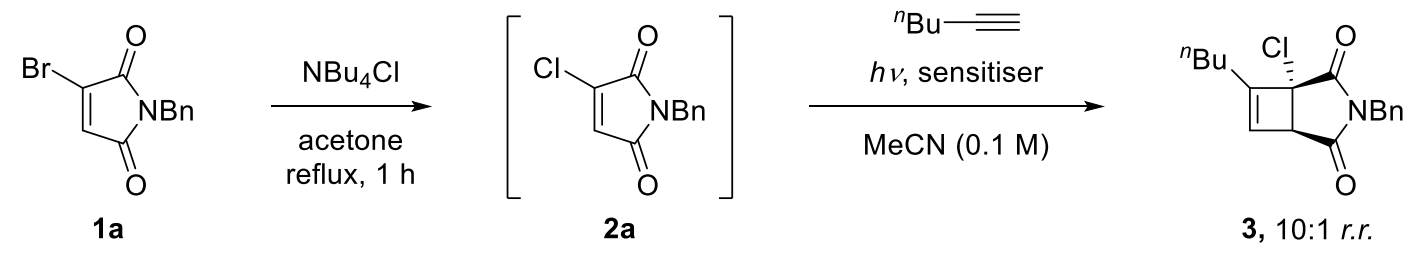

\begin{tabular}{|c|c|c|c|c|c|}
\hline Entry $^{a}$ & Sensitiser (mol\%) & $\mathbf{E}_{\mathrm{T}}(\mathbf{k J} / \mathbf{m o l})^{\mathrm{b}}$ & Conversion (\%) & Time (h) & Yield of $3(\%)^{c}$ \\
\hline 1 & None & - & 100 & 20 & 75 \\
\hline 2 & 3-MeOTX (1) & 283 & 100 & 1.5 & 74 \\
\hline 3 & $\operatorname{ITX}(1)$ & 266 & 100 & 1.5 & 75 \\
\hline
\end{tabular}

${ }^{a}$ See General Procedure 3 for reaction conditions. ${ }^{\mathrm{b}}$ Triplet energy values taken from the literature. ${ }^{7 \mathrm{c}}$ Yields calculated by ${ }^{1} \mathrm{H}$ NMR using trimethoxybenzene as an internal standard. 


\subsection{Substrate Scope}

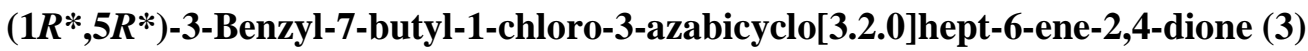
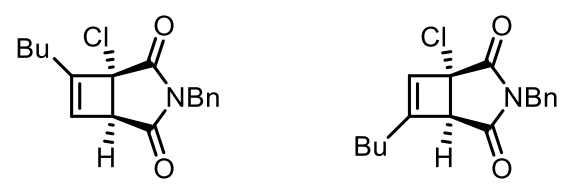

$15: 1$ r.r.

General procedure 3 - Batch. Using crude 2a (18.8 mmol), ITX (46 mg, $0.19 \mathrm{mmol})$, and 1-hexyne $(6.43 \mathrm{~mL}, 56.4 \mathrm{mmol})$. The reaction was exposed to UVA conditions for 90 minutes. Purification by column chromatography (99/1 petroleum ether/ethyl acetate) gave the title compound (4.28 g, 75\%) as a pale yellow oil. The r.r. was enhanced from 10:1 to 15:1 during purification.

${ }^{1} \mathbf{H}$ NMR $\left(400 \mathrm{MHz}, \mathrm{CDCl}_{3}\right) \delta_{\mathrm{H}}$ (major regioisomer reported) $7.36-7.26(\mathrm{~m}, 5 \mathrm{H}), 6.20(\mathrm{~d}, J=1.5 \mathrm{~Hz}$, $1 \mathrm{H}), 4.74-4.59$ (m, 2H), $3.69(\mathrm{~d}, J=1.0 \mathrm{~Hz}, 1 \mathrm{H}), 2.20(\mathrm{dq}, J=6.5,2.0 \mathrm{~Hz}, 2 \mathrm{H}), 1.55-1.37(\mathrm{~m}, 2 \mathrm{H})$, $1.34-1.21(\mathrm{~m}, 2 \mathrm{H}), 0.88(\mathrm{t}, J=7.5 \mathrm{~Hz}, 3 \mathrm{H})$. See spectrum.

${ }^{13} \mathbf{C}$ NMR $\left(100 \mathrm{MHz}, \mathrm{CDCl}_{3}\right) \delta_{\mathrm{C}}$ (major regioisomer reported) $172.6,171.0,154.7,135.2,129.9,128.7$, 128.5, 128.1, 63.9, 53.4, 42.6, 27.4, 26.8, 22.2, 13.7. See spectrum.

IR $v_{\max }: 2957,2931,1778,1711,1429,1382,1340,1171,855,699 \mathrm{~cm}^{-1}$.

HRMS (ESI) m/z: [(M+Na) $\left.{ }^{+}\right]$calcd for $\mathrm{C}_{17} \mathrm{H}_{18} \mathrm{ClNO}_{2} 326.0924$, found 326.0909.

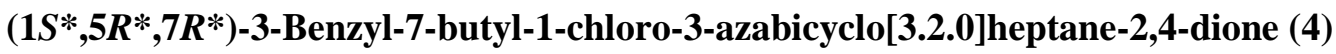
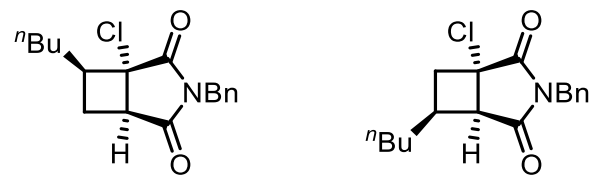

10:1 r.r.

General procedure 3 - Batch. Using crude 2a (16.9 mmol), ITX (46 mg, $0.19 \mathrm{mmol})$, and 1-hexyne $(6.43 \mathrm{~mL}, 56.4 \mathrm{mmol})$. The reaction was exposed to UVA conditions for 90 minutes.

General procedure 4. Using the crude material from the previous step $(14.1 \mathrm{mmol}), 5 \% \mathrm{Pd} / \mathrm{C}(1.00 \mathrm{~g}$, $20 \% \mathrm{w} / \mathrm{w})$ and methanol $(150 \mathrm{~mL})$. Purification by column chromatography (99/1 petroleum ether/ethyl acetate) gave the title compound $(3.83 \mathrm{~g}, 67 \%)$ as a colourless oil. The configuration of the major diastereomer was assigned by analogy to the known selectivities observed in hydrogenations of bicyclic cyclobutenes, ${ }^{8}$ and confirmed by NOE studies ( see spectra). 
${ }^{1} \mathbf{H}$ NMR $\left(400 \mathrm{MHz}, \mathrm{CDCl}_{3}\right) \delta_{\mathrm{H}}$ (major regioisomer reported) $7.37-7.20(\mathrm{~m}, 5 \mathrm{H}), 4.67(\mathrm{~m}, 2 \mathrm{H}), 3.23$ $(\mathrm{dd}, J=10.5,5.5 \mathrm{~Hz}, 1 \mathrm{H}), 2.85(\mathrm{dt}, J=16.0,8.0 \mathrm{~Hz}, 1 \mathrm{H}), 2.75(\mathrm{dt}, J=12.5,10.5 \mathrm{~Hz}, 1 \mathrm{H}), 1.56-1.48$ (m, 1H), 1.39 (ddd, $J=12.5,8.0,6.0 \mathrm{~Hz}, 1 \mathrm{H}), 1.27-1.06(\mathrm{~m}, 4 \mathrm{H}), 1.05-0.93(\mathrm{~m}, 1 \mathrm{H}), 0.76$ (t, $J=7.0$ Hz, 3H). See spectrum.

${ }^{13} \mathbf{C} \mathbf{N M R}\left(100 \mathrm{MHz}, \mathrm{CDCl}_{3}\right) \delta_{\mathrm{C}}$ (major regioisomer reported) $175.8,172.8,135.4,128.8,128.7,128.2$, 62.7, 46.3, 44.9, 42.9, 31.6, 28.5, 26.9, 22.3, 13.8. See spectrum.

IR $v_{\max }: 2932,1714,1385,1341,699 \mathrm{~cm}^{-1}$.

HRMS (ESI) m/z: [(M+H) $]$ calcd for $\mathrm{C}_{17} \mathrm{H}_{20} \mathrm{ClNO}_{2} 306.1261$, found 306.1270 .

$\left(1 S^{*}, 5 R^{*}, 7 R^{*}\right)-3-M e t h y l-7-b u t y l-1-c h l o r o-3-a z a b i c y c l o[3.2 .0]$ heptane-2,4-dione (5)
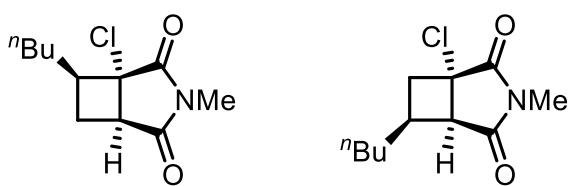

$12: 1$ r.r.

General procedure 3 - Batch. Using crude $2 \mathbf{b}(6.8 \mathrm{mmol})$, ITX (18 $\mathrm{mg}, 0.070 \mathrm{mmol})$, and 1-hexyne $(2.30 \mathrm{~mL}, 20.0 \mathrm{mmol})$. The reaction was exposed to UVA conditions for 4.5 hours.

General procedure 4. Using the crude material from the previous step $(5.0 \mathrm{mmol}), 5 \% \mathrm{Pd} / \mathrm{C}(260 \mathrm{mg}$, $20 \% \mathrm{w} / \mathrm{w})$ and methanol $(70 \mathrm{~mL})$, with a reaction time of 18 hours. Purification by column chromatography ( $95 / 5$ petroleum ether/ethyl acetate) gave the title compound ( $800 \mathrm{mg}, 51 \%$ ) as a colourless oil.

${ }^{1} \mathbf{H}$ NMR $\left(400 \mathrm{MHz}, \mathrm{CDCl}_{3}\right) \delta_{\mathrm{H}}$ (major regioisomer reported) $3.25-3.18(\mathrm{~m}, 1 \mathrm{H}), 2.98(\mathrm{~s}, 3 \mathrm{H}), 2.91$ $-2.81(\mathrm{~m}, 1 \mathrm{H}), 2.76(\mathrm{dt}, J=12.0,10.5 \mathrm{~Hz}, 1 \mathrm{H}), 1.67-1.54(\mathrm{~m}, 1 \mathrm{H}), 1.48$ (ddd, $J=12.0,8.0,6.0 \mathrm{~Hz}$, 1H), $1.29-1.06$ (m, 5H), $0.83-0.74(\mathrm{~m}, 3 \mathrm{H})$. See spectrum.

${ }^{13} \mathbf{C}$ NMR $\left(100 \mathrm{MHz}, \mathrm{CDCl}_{3}\right) \delta_{\mathrm{C}}$ (major regioisomer reported) $175.5,172.4,62.2,45.3,44.2,31.0,28.0$, 26.1, 24.7, 21.6, 13.2. See spectrum.

IR $v_{\max }: 2955,2931,2858,1783,1699,1426 \mathrm{~cm}^{-1}$.

HRMS (ESI) m/z: [(M+Na) $\left.{ }^{+}\right]$calcd for $\mathrm{C}_{11} \mathrm{H}_{16} \mathrm{ClNO}_{2} 252.0762$, found 252.0760 . 
$\left(1 S^{*}, 5 R^{*}, 7 R^{*}\right)$-7-Butyl-1-chloro-3-azabicyclo[3.2.0]heptane-2,4-dione (6)
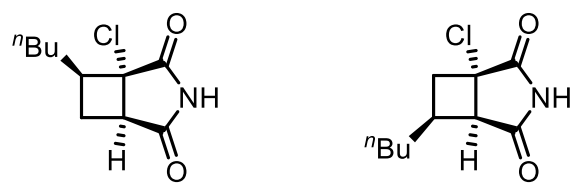

$11: 1$ r.r.

General procedure 3 - Batch. Using crude $2 c$ (7.5 mmol), ITX (19 mg, $0.075 \mathrm{mmol})$, and 1-hexyne $(2.48 \mathrm{~mL}, 22.0 \mathrm{mmol})$. The reaction was exposed to UVA conditions for 4.5 hours.

General procedure 4. Using the crude material from the previous step $(5.19 \mathrm{mmol}), 5 \% \mathrm{Pd} / \mathrm{C}(260 \mathrm{mg}$, $20 \% \mathrm{w} / \mathrm{w})$ and methanol $(75 \mathrm{~mL})$, with a reaction time of 24 hours. Purification by column chromatography (90/10 petroleum ether/ethyl acetate) gave the title compound ( $906 \mathrm{mg}, 56 \%$ ) as a colourless oil.

${ }^{1} \mathbf{H}$ NMR $\left(400 \mathrm{MHz}, \mathrm{CDCl}_{3}\right) \delta_{\mathrm{H}}$ (major regioisomer reported) $9.19(\mathrm{~s}, 1 \mathrm{H}), 3.36-3.28(\mathrm{~m}, 1 \mathrm{H}),(3.00$ $-2.90(\mathrm{~m}, 1 \mathrm{H}), 2.88(\mathrm{dt}, J=12.0,10.5 \mathrm{~Hz}, 1 \mathrm{H}), 1.74(\mathrm{dtt}, J=12.0,8.0,6.0 \mathrm{~Hz}, 2 \mathrm{H}), 1.39-1.16(\mathrm{~m}$, $5 \mathrm{H}), 0.94-0.86(\mathrm{~m}, 3 \mathrm{H})$. See spectrum.

${ }^{13} \mathbf{C}$ NMR $\left(100 \mathrm{MHz}, \mathrm{CDCl}_{3}\right) \delta_{\mathrm{C}}$ (major regioisomer reported) 175.7, 172.6, 63.1, 45.4, 45.3, 31.0, 27.9, 26.3, 21.7, 13.2. See spectrum.

IR $v_{\max }: 3214,3061,2955,2928,2855,1772,1708,1493,1451,1171,1042 \mathrm{~cm}^{-1}$.

HRMS (ESI) m/z: [(M+Na) $\left.{ }^{+}\right]$calcd for $\mathrm{C}_{10} \mathrm{H}_{14} \mathrm{ClNO}_{2} 238.0605$, found 238.0613.

$\left(1 S^{*}, 5 R^{*}, 7 R^{*}\right)$-3-Benzyl-1-chloro-7-(trimethylsilyl)-3-azabicyclo[3.2.0]heptane-2,4-dione (7)
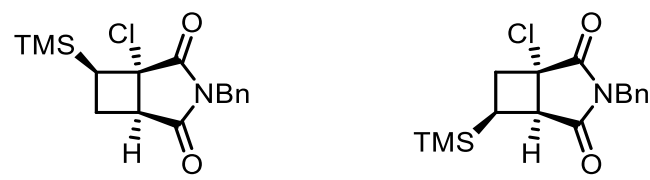

$4: 1$ r.r.

General procedure 3 - Batch. Using crude 2a (16.9 mmol), ITX (48 mg, $0.19 \mathrm{mmol}$ ), and ethynyltrimethylsilane $(7.80 \mathrm{~mL}, 56.4 \mathrm{mmol})$. The reaction was exposed to UVA conditions for 2.5 hours.

General procedure 4. Using the crude material from the previous step $(16.9 \mathrm{mmol}), 5 \% \mathrm{Pd} / \mathrm{C}(1.00 \mathrm{~g}$, $20 \% \mathrm{w} / \mathrm{w})$ and methanol $(150 \mathrm{~mL})$, with a reaction time of 18 hours. Purification by column chromatography (99/1 petroleum ether/ethyl acetate) gave the title compound $(2.77 \mathrm{~g}, 44 \%)$ as a colourless oil. 
${ }^{1} \mathbf{H}$ NMR $\left(400 \mathrm{MHz}, \mathrm{CDCl}_{3}\right) \delta_{\mathrm{H}}$ (major regioisomer reported) $7.43-7.27(\mathrm{~m}, 5 \mathrm{H}), 4.81-4.59(\mathrm{~m}, 2 \mathrm{H})$, $3.57(\mathrm{dd}, J=11.0,6.0 \mathrm{~Hz}, 1 \mathrm{H}), 2.81-2.68(\mathrm{~m}, 1 \mathrm{H}), 2.60-2.51(\mathrm{~m}, 1 \mathrm{H}), 1.73(\mathrm{ddd}, J=12.0,9.5,6.0$ $\mathrm{Hz}, 1 \mathrm{H}), 0.10$ (s, 9H). See spectrum.

${ }^{13} \mathbf{C} \mathbf{N M R}\left(100 \mathrm{MHz}, \mathrm{CDCl}_{3}\right) \delta_{\mathrm{C}}$ (major regioisomer reported) $175.5,174.3,135.1,129.1,128.8,128.2$, 62.0, 48.4, 43.1, 36.3, 21.4, 0.1. See spectrum.

IR $v_{\max }: 2954,1711,1384,1249,837,697 \mathrm{~cm}^{-1}$.

HRMS (ESI) m/z: [(M+Na) $\left.{ }^{+}\right]$calcd for $\mathrm{C}_{16} \mathrm{H}_{20} \mathrm{ClNO}_{2} 339.1290$, found 339.1273.

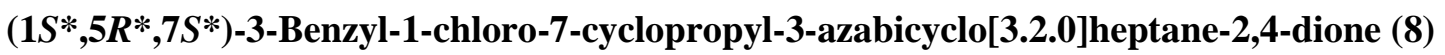

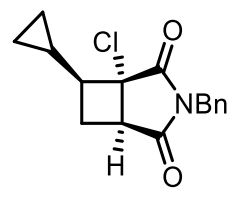

$>20: 1$

General procedure 3 - Batch. Using crude 2a (13.5 mmol), ITX (37 mg, $0.15 \mathrm{mmol}$ ), and cyclopropyl acetylene $(3.81 \mathrm{~mL}, 45.0 \mathrm{mmol})$. The reaction was exposed to UVA conditions for 90 minutes. The product was purified by column chromatography (98/2 petroleum ether/ethyl acetate) to give the cyclobutene $(3.00 \mathrm{~g}, 56 \%)$ as a colourless oil in a 9:1 regioisomeric ratio.

Modified General procedure 4. Using the material from the previous step ( $2.50 \mathrm{~g}, 8.69 \mathrm{mmol}), 5 \% \mathrm{Pt} / \mathrm{C}$ $(500 \mathrm{mg}, 20 \% \mathrm{w} / \mathrm{w})$ and methanol $(87 \mathrm{~mL})$, with a reaction time of 18 hours. A small amount of the $n$ propyl product of cyclopropane cleavage was observed (8\%) but this could be separated by column chromatography [Note: when using $\mathrm{Pd} / \mathrm{C}$ in place of $\mathrm{Pt} / \mathrm{C}$, the ratio of cyclopropyl:n-propyl products was 1:1]. Purification by column chromatography (98/2 petroleum ether/ethyl acetate) gave the title compound $(1.88 \mathrm{~g}, 75 \%)$ as a colourless oil (the minor regioisomer was removed during purification). $42 \%$ overall yield.

${ }^{1} \mathbf{H}$ NMR $\left(500 \mathrm{MHz}, \mathrm{CDCl}_{3}\right) \delta_{\mathrm{H}} 7.48-7.29(\mathrm{~m}, 5 \mathrm{H}), 4.85-4.68(\mathrm{~m}, 2 \mathrm{H}), 3.36-3.23(\mathrm{~m}, 1 \mathrm{H}),, 2.82-$ $2.59(\mathrm{~m}, 2 \mathrm{H}), 1.51$ (ddd, $J=12.5,7.5,5.5 \mathrm{~Hz}, 1 \mathrm{H}), 0.69$ (dddd, $J=8.0,5.0 \mathrm{~Hz}, 1 \mathrm{H}), 0.57-0.47$ (m, $1 \mathrm{H}$ ), 0.29 (dddd, $J=9.0,8.0,6.0,5.0 \mathrm{~Hz}, 1 \mathrm{H}), 0.19$ (ddt, $J=9.5,6.0,5.0 \mathrm{~Hz}, 1 \mathrm{H}$ ), 0.1 (ddd, $J=10.5$, 9.5, $5.0 \mathrm{~Hz}, 1 \mathrm{H})$. See spectrum.

${ }^{13} \mathbf{C}$ NMR $\left(125 \mathrm{MHz}, \mathrm{CDCl}_{3}\right) \delta_{\mathrm{C}} 175.7,172.8,135.2,129.0,128.8,128.2,62.7,50.0,44.5,43.0,24.1$, 10.8, 2.3. See spectrum.

IR $v_{\max }: 3004,1708,1383,1339,1177,696 \mathrm{~cm}^{-1}$.

HRMS HRMS (ESI) m/z: [(M+Na) $)^{+}$calcd for $\mathrm{C}_{16} \mathrm{H}_{16} \mathrm{ClNO}_{2} 312.0762$, found 312.0760 . 

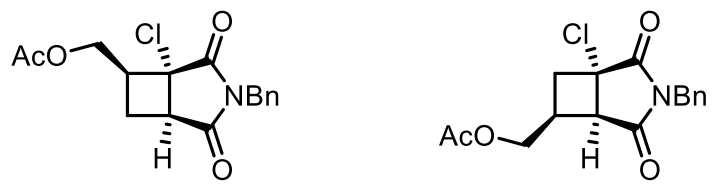

$4: 1$ r.r.

General procedure 3 - Batch. Using crude 2a (16.9 mmol), ITX (48 mg, $0.19 \mathrm{mmol})$, and propargyl alcohol (3.28 mL, $56.4 \mathrm{mmol})$. The reaction was exposed to UV for 90 minutes. Purification by column chromatography (5/2 petroleum ether/ethyl acetate) gave the cyclobutene $(3.65 \mathrm{~g}, 70 \%)$ as a colourless oil.

To a solution of the cyclobutene alcohol $(2.87 \mathrm{~g}, 10.3 \mathrm{mmol})$ in DCM $(41 \mathrm{~mL})$ at r.t. was added 4-(dimethylamino)pyridine (126 mg, $1.03 \mathrm{mmol})$ and pyridine $(0.81 \mathrm{~mL}, 10 \mathrm{mmol})$. The solution was stirred for 5 minutes before adding acetic anhydride $(1.97 \mathrm{~mL}, 20.3 \mathrm{mmol})$ and stirring at r.t. for a further 16 hours. The solution was quenched with $1 \mathrm{M}$ aqueous $\mathrm{HCl}(10 \mathrm{~mL})$ and extracted with DCM $(3 \times 10 \mathrm{~mL})$. The organic layers were combined and dried over $\mathrm{MgSO}_{4}$, and solvent removed in vacuo. Purification by column chromatography (70/30 petroleum ether/ethyl acetate) gave the cyclobutene acetate $(2.48 \mathrm{~g}, 78 \%)$ as an orange oil.

General procedure 4. Using the material from the previous step (200 mg, $0.624 \mathrm{mmol}$ ), 5\% Pd/C (40 $\mathrm{mg}, 20 \% \mathrm{w} / \mathrm{w})$ and methanol $(6.3 \mathrm{~mL})$. Purification by column chromatography (60/40 petroleum ether/ethyl acetate) gave the title compound (177 mg, 88\%) as a colourless oil. $48 \%$ overall yield.

${ }^{1} \mathbf{H} \mathbf{N M R}\left(400 \mathrm{MHz}, \mathrm{CDCl}_{3}\right) \delta_{\mathrm{H}}$ (major regioisomer reported) $7.38-7.19(\mathrm{~m}, 5 \mathrm{H}), 4.77-4.59(\mathrm{~m}, 2 \mathrm{H})$, $4.11-4.00(\mathrm{~m}, 2 \mathrm{H}), 3.38-3.30(\mathrm{~m}, 1 \mathrm{H}), 3.25$ (ddt, $J=10.5,8.5,5.0,1 \mathrm{H}), 2.83-2.69(\mathrm{~m}, 1 \mathrm{H}),$, $-1.76(\mathrm{~m}, 4 \mathrm{H})$. See spectrum.

${ }^{13} \mathbf{C}$ NMR $\left(100 \mathrm{MHz}, \mathrm{CDCl}_{3}\right) \delta_{\mathrm{C}}$ (major regioisomer reported) $175.0,172.4,170.3,135.2,128.9,128.7$, 128.2, 61.5, 60.8, 44.9, 44.6, 43.1, 22.9, 20.3. See spectrum.

IR $v_{\max }: 2947,1742,1711,1386,1232,1042,699 \mathrm{~cm}^{-1}$.

HRMS (ESI) m/z: [(M+H) $]$ calcd for $\mathrm{C}_{16} \mathrm{H}_{16} \mathrm{NO}_{4} 322.0846$, found 322.0828 . 

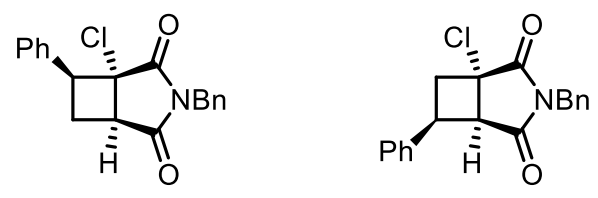

$7: 1$ r.r.

General procedure 3 - Syringe pump. Using crude 2a (16.9 mmol), ITX (48 $\mathrm{mg}, 0.19 \mathrm{mmol})$, and phenylacetylene $(6.2 \mathrm{~mL}, 56 \mathrm{mmol})$. Phenylacetylene was added over 2 hours under UVA irradiation, and irradiation was continued for a further 3 hours. Purification by column chromatography (100\% petroleum ether, to remove excess phenylacetylene, followed by $90 / 10$ petroleum ether/ethyl acetate) gave a mixture of cyclobutene and the [2+2]-dimer of $N$-benzyl maleimide, which was used in the following step without further purification.

General procedure 4. Using the material from the previous step $(16.9 \mathrm{mmol}), 5 \% \mathrm{Pd} / \mathrm{C}(1.00 \mathrm{~g}, 20 \%$ w/w) and methanol $(150 \mathrm{~mL})$. Purification by column chromatography $(98 / 2$ petroleum ether/ethyl acetate) gave the title compound $(1.20 \mathrm{~g}, 27 \%)$ as a colourless oil.

${ }^{1} \mathbf{H}$ NMR $\left(400 \mathrm{MHz}, \mathrm{CDCl}_{3}\right) \delta_{\mathrm{H}}$ (major regioisomer reported) $7.36-7.22(\mathrm{~m}, 8 \mathrm{H}), 7.04-6.96(\mathrm{~m}, 2 \mathrm{H})$, $4.72-4.56(\mathrm{~m}, 2 \mathrm{H}), 4.28(\mathrm{dd}, J=11.0,8.5,1 \mathrm{H}),, 3.49(\mathrm{dd}, J=10.5,5.5,1 \mathrm{H}), 3.15-3.01(\mathrm{~m}, 1 \mathrm{H}), 2.27$ (ddd, $J=13.0,8.5,5.5,1 \mathrm{H})$. See spectrum.

${ }^{13} \mathbf{C}$ NMR $\left(125 \mathrm{MHz}, \mathrm{CDCl}_{3}\right) \delta_{\mathrm{C}}$ (major regioisomer reported) 175.3, 171.7, 135.2, 135.2, 129.1, 128.8, 128.6, 128.2, 128.1, 127.1, 64.1, 50.7, 44.6, 42.9, 24.9. See spectrum.

IR $v_{\max }: 3031,1708,1384,1339,1163,694 \mathrm{~cm}^{-1}$.

HRMS (ESI) m/z: [(M+Na) $\left.{ }^{+}\right]$calcd for $\mathrm{C}_{19} \mathrm{H}_{16} \mathrm{ClNO}_{2} 348.0762$, found 348.0774.

Ethyl $\left(1 R^{*}, 5 R^{*}, 6 S^{*}\right)$-3-benzyl-5-chloro-2,4-dioxo-3-azabicyclo[3.2.0]heptane-6-carboxylate (11)
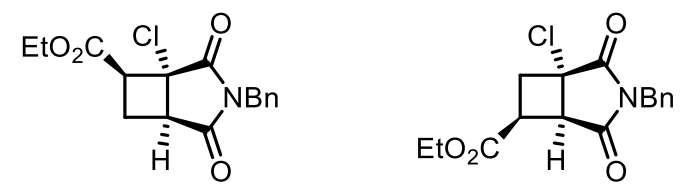

$4: 1$ r.r.

General procedure 3 - Syringe pump. Using crude 2a (16.9 mmol), ITX (46 mg, $0.19 \mathrm{mmol})$, and ethyl propiolate $(5.70 \mathrm{~mL}, 56.4 \mathrm{mmol})$. Ethyl propiolate was added over 2 hours under UVA irradiation, and irradiation was continued for a further 2 hours. Purification by column chromatography (95/5 to 90/10 petroleum ether/ethyl acetate) gave the cyclobutene product (2.64 g, 44\%) as a yellow solid. 
General procedure 4. Using the material from the previous step ( $2.64 \mathrm{~g}, 8.26 \mathrm{mmol}), 5 \% \mathrm{Pd} / \mathrm{C}(500 \mathrm{mg}$, $20 \% \mathrm{w} / \mathrm{w})$ and methanol $(83 \mathrm{~mL})$. Purification by column chromatography (95/5 to 90/10 petroleum ether/ethyl acetate) gave the title compound $(2.11 \mathrm{~g}, 79 \%)$ as a yellow oil. $35 \%$ overall yield.

${ }^{1} \mathrm{H}$ NMR $\left(400 \mathrm{MHz}, \mathrm{CDCl}_{3}\right) \delta_{\mathrm{H}}$ (major regioisomer reported) $7.44-7.29(\mathrm{~m}, 5 \mathrm{H}), 4.80-4.72(\mathrm{~m}, 1 \mathrm{H})$, $4.22-4.09$ (m, 2H), $3.81-3.73(\mathrm{~m}, 1 \mathrm{H}), 3.42$ (ddd, $J=10.5,6.0,3.5 \mathrm{~Hz}, 1 \mathrm{H}), 2.87$ (ddd, $J=13.0$, $10.5,2.5 \mathrm{~Hz}, 1 \mathrm{H}), 2.35(\mathrm{ddd}, J=13.0,8.0,6.0 \mathrm{~Hz}, 1 \mathrm{H}), 1.27(\mathrm{t}, J=7.0,3 \mathrm{H})$. See spectrum.

${ }^{13} \mathbf{C ~ N M R}\left(100 \mathrm{MHz}, \mathrm{CDCl}_{3}\right) \delta_{\mathrm{C}}$ (major regioisomer reported) $174.3,171.8,169.0,134.9,128.8,128.6$, 128.2, 62.0, 60.9, 48.2, 45.0, 43.3, 22.5, 14.0. See spectrum.

IR $v_{\max }: 2987,1715,1385,1342,1202,699 \mathrm{~cm}^{-1}$.

HRMS (ESI) m/z: [(M+H) $]$ calcd for $\mathrm{C}_{16} \mathrm{H}_{16} \mathrm{ClNO}_{4} 322.0841$, found 322.0832 .

$\left(3 \mathrm{a} S^{*}, 3 \mathrm{~b} S^{*}, 6 \mathrm{a} R^{*}, 6 \mathrm{~b} R^{*}\right)$-2-benzyl-3a-chlorohexahydrocyclopenta[3,4]cyclobuta[1,2-c]pyrrole1,3(2H,3a $H)$-dione (12)
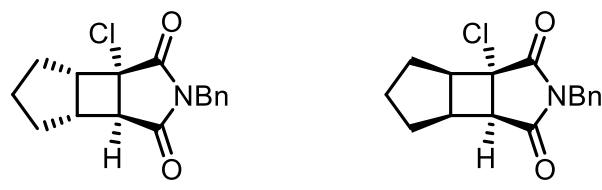

$12: 1$ r.r.

General procedure 3 - Batch. Using crude 2a (13.5 mmol), ITX (46 mg, $0.19 \mathrm{mmol})$, and cyclopentene $(3.98 \mathrm{~mL}, 45.0 \mathrm{mmol})$. The reaction was exposed to UVA conditions for 90 minutes. Purification by column chromatography (90/10 petroleum ether/ethyl acetate) gave the title compound (3.39 g, 78\%) as a colourless solid. The configuration of major diastereomer was assigned by analogy to the known selectivities observed in related $[2+2]$ photocycloadditions, ${ }^{9}$ and confirmed by NOE studies (see spectra).

${ }^{1} \mathbf{H}$ NMR $\left(400 \mathrm{MHz}, \mathrm{CDCl}_{3}\right) \delta_{\mathrm{H}}$ (major diastereoisomer reported) $7.43-7.29(\mathrm{~m}, 5 \mathrm{H}), 4.82-4.69(\mathrm{~m}$, 2H), $3.07-3.00(\mathrm{~m}, 1 \mathrm{H}), 2.79$ (dd, $J=3.5,1.0 \mathrm{~Hz}, 1 \mathrm{H}$ ), $2.70(\mathrm{td}, J=6.0,3.5 \mathrm{~Hz}, 1 \mathrm{H}), 2.27-2.18(\mathrm{~m}$, 1H), $2.04-1.95$ (m, 1H) 1.93 (dd, $J=11.0,3.5 \mathrm{~Hz}, 1 \mathrm{H}), 1.88-1.76(\mathrm{~m}, 1 \mathrm{H}), 1.70$ (ddd, $J=13.5,6.5$, $1.0 \mathrm{~Hz}, 1 \mathrm{H}), 1.64(\mathrm{dd}, J=12.5,6.0 \mathrm{~Hz}, 1 \mathrm{H})$. See spectrum.

${ }^{13} \mathbf{C}$ NMR $\left(100 \mathrm{MHz}, \mathrm{CDCl}_{3}\right) \delta_{\mathrm{C}}$ (major diastereoisomer reported) $175.7,175.6,135.3,128.8,128.4$, 128.1, 60.9, 52.2, 45.5, 43.0, 41.5, 32.9, 29.0, 24.8. See spectrum.

IR $v_{\max }: 2957,2931,1779,1712,1383,700 \mathrm{~cm}^{-1}$.

HRMS (ESI) m/z: [(M+Na) $)^{+}$calcd for $\mathrm{C}_{16} \mathrm{H}_{16} \mathrm{ClNO}_{2} 312.0767$, found 312.0763.

$\operatorname{Mp}\left(\mathrm{CDCl}_{3}\right): 77-80{ }^{\circ} \mathrm{C}$. 
tert-Butyl $\left(1 R^{*}, 5 R^{*}\right)$-3-benzyl-5-chloro-2,4-dioxo-3-azaspiro[bicyclo[3.2.0]heptane-6,4'piperidine]-1'-carboxylate (13)

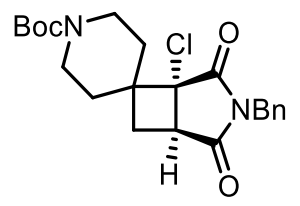

General procedure 3 - Syringe pump. Using crude 2a (5.6 mmol), ITX (16 mg, $0.06 \mathrm{mmol})$, and 1-boc4-methylenepiperidine (1.86 g, $9.42 \mathrm{mmol}$ ). 1-Boc-4-methylenepiperidine was added over 90 minutes under UVA irradiation, and irradiation was continued for a further 90 minutes. Purification by column chromatography (95/5 to 90/10 petroleum ether/ethyl acetate) gave the title compound $(1.17 \mathrm{~g}, 45 \%)$ as a yellow oil, and the regioisomeric product (581 mg, 22\%).

${ }^{1} \mathbf{H}$ NMR $\left(400 \mathrm{MHz}, \mathrm{CDCl}_{3}\right) \delta_{\mathrm{H}} 7.36-7.16(\mathrm{~m}, 5 \mathrm{H}), 4.74-4.54(\mathrm{~m}, 2 \mathrm{H}), 3.94-3.69(\mathrm{~m}, 1 \mathrm{H}), 3.71-$ $3.57(\mathrm{~m}, 1 \mathrm{H}), 3.29-3.18(\mathrm{~m}, 1 \mathrm{H}), 3.12-2.85(\mathrm{~m}, 1 \mathrm{H}), 2.63(\mathrm{ddd}, J=14.0,10.5,3.0 \mathrm{~Hz}, 1 \mathrm{H}), 2.47-$ $2.37(\mathrm{~m}, 1 \mathrm{H}), 1.88-1.78(\mathrm{~m}, 1 \mathrm{H}), 1.71-1.51(\mathrm{~m}, 3 \mathrm{H}), 1.37(\mathrm{~s}, 9 \mathrm{H}), 1.08-1.00(\mathrm{~m}, 1 \mathrm{H})$. See spectrum. ${ }^{13} \mathrm{C}$ NMR $\left(100 \mathrm{MHz}, \mathrm{CDCl}_{3}\right) \delta_{\mathrm{C}} 175.6,173.0,154.5,135.2,128.8,128.8,128.3,79.9,67.0,43.53$, 43.45, 43.0, 39.7, 34.1, 33.9, 32.5, 28.4. See spectrum.

IR $v_{\max }: 2974,2938,1712,1687,1171,732,698 \mathrm{~cm}^{-1}$.

HRMS (ESI) m/z: [(M+Na) $\left.)^{+}\right]$calcd for $\mathrm{C}_{22} \mathrm{H}_{27} \mathrm{NO}_{4} 441.1552$, found 441.1563 .

$\left(3 \mathrm{a} R^{*}, 4 \mathrm{a} S^{*}, 7 \mathrm{a} S^{*}\right)-4 \mathrm{a}-c h l o r o-6-m e t h y l t e t r a h y d r o f u r o\left[2^{\prime}, 3^{\prime}: 1,4\right]$ cyclobuta[1,2-c]pyrrole$5,7(2 \mathrm{H}, 6 \mathrm{H})$-dione (14)

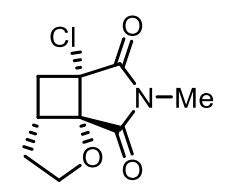

General procedure 3 - Batch. Using $\mathbf{S 1}(990 \mathrm{mg}$, $4.59 \mathrm{mmol}$ ). The reaction was exposed to UV light for 60 minutes. Purification by column chromatography (3/1 to $3 / 2$ petroleum ether/ethyl acetate) gave the title compound $(897 \mathrm{mg}, 91 \%)$ as a colourless crystalline solid.

${ }^{1} \mathbf{H}$ NMR $\left(400 \mathrm{MHz}, \mathrm{CDCl}_{3}\right) \delta_{\mathrm{H}} 4.55(\mathrm{ddt}, J=10.5,8.5,2.0 \mathrm{~Hz}, 1 \mathrm{H}), 4.37-4.23(\mathrm{~m}, 1 \mathrm{H}), 3.17-3.03$ $(\mathrm{m}, 4 \mathrm{H}), 2.94-2.84(\mathrm{~m}, 1 \mathrm{H}), 2.26-2.07(\mathrm{~m}, 2 \mathrm{H}), 2.04-1.92(\mathrm{~m}, 1 \mathrm{H})$. See spectrum.

${ }^{13}$ C NMR $\left(100 \mathrm{MHz}, \mathrm{CDCl}_{3}\right) \delta_{\mathrm{C}} 173.1,172.6,86.1,72.0,59.6,41.7,35.7,31.4,25.6 . \underline{\text { See spectrum. }}$ IR $v_{\max }: 2994,1787,1712,1375,1183,1026,944,696 \mathrm{~cm}^{-1}$. 
HRMS (ESI) m/z: $\left[(\mathrm{M}+\mathrm{Na})^{+}\right]$calcd for $\mathrm{C}_{9} \mathrm{H}_{10} \mathrm{ClNO}_{3} 238.0241$, found 238.0245.

Mp (DCM): $92-93{ }^{\circ} \mathrm{C}$. 


\section{Photoredox-Catalysed Dechlorinative Functionalisations}

\subsection{Control Reactions}

Table S2. Control studies of conditions

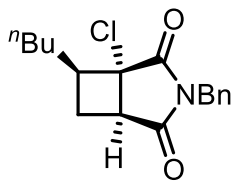

4

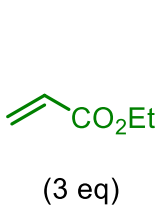

(3 eq)

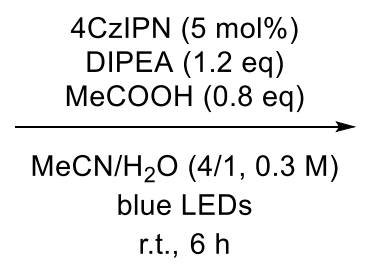

r.t., $6 \mathrm{~h}$

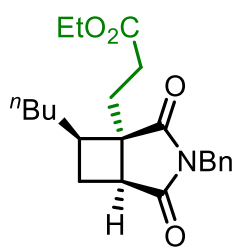

24

\begin{tabular}{|c|cccccc|c|}
\hline Entry & 4CzIPN & DIPEA & Water & MeCOOH & Light & N2 Sparge & Yield of 24 (\%) \\
\hline $\mathbf{1}$ & $\checkmark$ & $\checkmark$ & $\checkmark$ & $\checkmark$ & $\checkmark$ & $\checkmark$ & 75 \\
$\mathbf{2}$ & $\boldsymbol{x}$ & $\checkmark$ & $\checkmark$ & $\checkmark$ & $\checkmark$ & $\checkmark$ & 0 \\
$\mathbf{3}$ & $\checkmark$ & $\boldsymbol{x}$ & $\checkmark$ & $\checkmark$ & $\checkmark$ & $\checkmark$ & 0 \\
$\mathbf{4}$ & $\checkmark$ & $\checkmark$ & $\boldsymbol{x}$ & $\checkmark$ & $\checkmark$ & $\checkmark$ & 52 \\
$\mathbf{5}$ & $\checkmark$ & $\checkmark$ & $\checkmark$ & $\boldsymbol{x}$ & $\checkmark$ & $\checkmark$ & 63 \\
$\mathbf{6}$ & $\checkmark$ & $\checkmark$ & $\checkmark$ & $\checkmark$ & $\times$ & $\checkmark$ & 0 \\
$\mathbf{7}$ & $\checkmark$ & $\checkmark$ & $\checkmark$ & $\checkmark$ & $\checkmark$ & $\times$ & 16 \\
$\mathbf{8}$ & $\checkmark$ & $\checkmark$ & $\boldsymbol{x}$ & $\boldsymbol{x}$ & $\checkmark$ & $\checkmark$ & 0 \\
$\mathbf{9}^{\mathrm{a}}$ & $\checkmark$ & $\checkmark$ & $\checkmark$ & $\checkmark$ & $\checkmark$ & $\checkmark$ & 67 (14) \\
$\mathbf{1 0}^{\mathrm{b}}$ & $\checkmark$ & $\checkmark$ & $\checkmark$ & $\checkmark$ & $\checkmark$ & $\checkmark$ & 47 \\
\hline
\end{tabular}

Reactions were performed on a 0.65 mmol scale. ${ }^{a}$ Using 3 equivalents of DIPEA. ${ }^{b}$ Using 3 equivalents of acetic acid. ${ }^{c}$ Number in parentheses in the yield of the hydrodehalogenation product. 


\subsection{Substrate Scope}

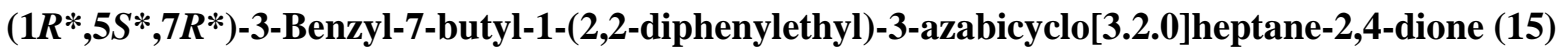
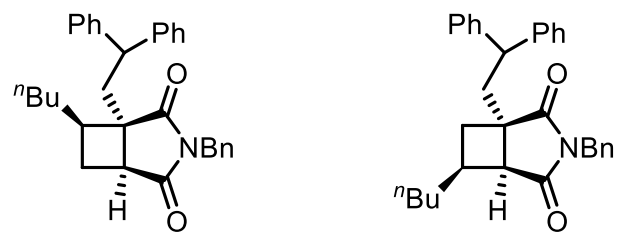

$18: 1$ r.r.

General procedure 5. Using chlorocyclobutane 4 (200 mg, $0.65 \mathrm{mmol}, 10: 1$ r.r.), 1,1-diphenylethylene (345 $\mu \mathrm{L}, 1.95 \mathrm{mmol})$, DIPEA (140 $\mu \mathrm{L}, 0.78 \mathrm{mmol})$, acetic acid (30 $\mu \mathrm{L}, 0.52 \mathrm{mmol}), 4 \mathrm{CzIPN}(26 \mathrm{mg}$, $5 \mathrm{~mol} \%)$ and $\mathrm{MeCN} /$ water $(4: 1,2.0 \mathrm{~mL})$, with a reaction time of 6 hours. Purification by column chromatography ( $99 / 1$ petroleum ether/ethyl acetate) gave the title compound ( $251 \mathrm{mg}, 86 \%$ ) as a colourless oil.

${ }^{1} \mathbf{H}$ NMR $\left(400 \mathrm{MHz}, \mathrm{CDCl}_{3}\right) \delta_{\mathrm{H}}$ (major regioisomer reported) $7.46-6.70(\mathrm{~m}, 15 \mathrm{H}), 4.57-4.44(\mathrm{~m}$, 2H), $3.71(\mathrm{dd}, J=10.5,5.0 \mathrm{~Hz}, 1 \mathrm{H}), 2.71(\mathrm{dd}, J=14.0,5.0 \mathrm{~Hz}, 1 \mathrm{H}), 2.45-2.34(\mathrm{~m}, 2 \mathrm{H}), 2.22(\mathrm{dd}, J=$ 9.5, 7.0 Hz, 1H), 2.05 (dd, $J=10.5,5.0 \mathrm{~Hz}, 1 \mathrm{H}), 1.42-1.32(\mathrm{~m}, 1 \mathrm{H}), 1.27$ (ddd, $J=12.5,7.5,5.5 \mathrm{~Hz}$, $1 \mathrm{H}), 1.11-0.91(\mathrm{~m}, 4 \mathrm{H}), 0.88-0.77(\mathrm{~m}, 1 \mathrm{H}), 0.72(\mathrm{t}, J=7.0 \mathrm{~Hz}, 3 \mathrm{H})$. See spectrum.

${ }^{13} \mathbf{C}$ NMR $\left(100 \mathrm{MHz}, \mathrm{CDCl}_{3}\right) \delta_{\mathrm{C}}$ (major regioisomer reported) $179.1,179.0,144.1,143.3,136.2,129.4$, 128.8, 128.6, 128.4, 128.1, 127.7, 127.4, 126.9, 126.4, 52.1, 47.8, 42.4, 42.3, 40.3, 38.8, 32.2, 29.0, 27.2, 22.4, 13.9. See spectrum.

IR $v_{\max }: 2929,2855,1697,1389,699 \mathrm{~cm}^{-1}$.

HRMS (ESI) m/z: $\left[(\mathrm{M}+\mathrm{H})^{+}\right]$calcd for $\mathrm{C}_{31} \mathrm{H}_{33} \mathrm{NO}_{2}$ 452.2584, found 452.2587 .

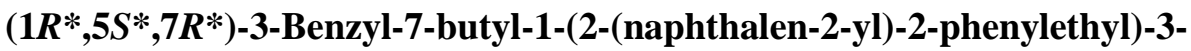
azabicyclo[3.2.0]heptane-2,4-dione (16)
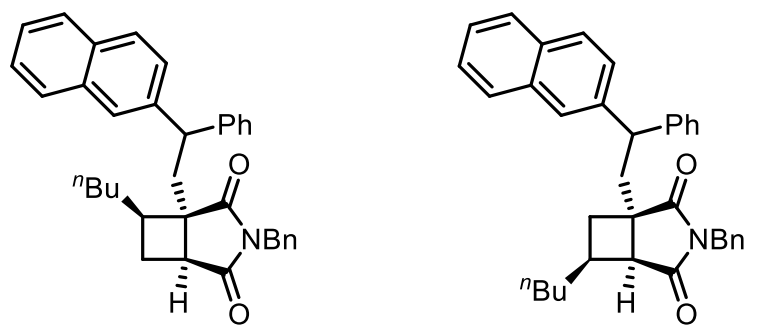

56:44 d.r

$9: 1$ r.r.

General procedure 5. Using chlorocyclobutane 4 (177 mg, $0.58 \mathrm{mmol}$, 10:1 r.r.), 2-(1phenylvinyl)naphthalene (452 mg, $1.95 \mathrm{mmol})$, DIPEA (140 $\mu \mathrm{L}, 0.78 \mathrm{mmol})$, acetic acid (30 $\mu \mathrm{L}, 0.52$ 
$\mathrm{mmol}$ ), 4CzIPN (26 mg, $5 \mathrm{~mol} \%$ ), and $\mathrm{MeCN} /$ water (4/1, $2.0 \mathrm{~mL}$ ) with a reaction time of 6 hours. Purification by column chromatography ( $98 / 2$ petroleum ether/ethyl acetate) gave the title compound (222 mg, 77\%) as a colourless oil.

${ }^{1} \mathbf{H}$ NMR $\left(400 \mathrm{MHz}, \mathrm{CDCl}_{3}\right) \delta_{\mathrm{H}}$ (mixture of diastereomers, $A+B$, of major regioisomer reported) 7.83 - 7.64 (m, 4H), $7.62-7.27$ (m, 9H), $7.22-7.11(\mathrm{~m}, 3 \mathrm{H}), 7.00-6.89$ (m, 1H), $4.70-4.42$ (m, 2H, A+B), 4.01 (ddd, $J=10.0,5.5,2.0 \mathrm{~Hz}, 1 \mathrm{H}, \mathrm{A}+\mathrm{B}), 2.95$ (ddd, $J=14.0,5.5,3.5 \mathrm{~Hz}, 1 \mathrm{H}, \mathrm{A}+\mathrm{B}), 2.64-$ $2.52(\mathrm{~m}, 1 \mathrm{H}, \mathrm{A}+\mathrm{B}), 2.52-2.41(\mathrm{~m}, 1 \mathrm{H}, \mathrm{A}+\mathrm{B}), 2.35(\mathrm{tdd}, J=9.5,7.5,6.0 \mathrm{~Hz}, 1 \mathrm{H}, \mathrm{A}+\mathrm{B}), 2.21(\mathrm{td}, J=$ 10.0, $5.0 \mathrm{~Hz}, 1 \mathrm{H}, \mathrm{A}+\mathrm{B}), 1.53-1.43(\mathrm{~m}, 1 \mathrm{H}, \mathrm{A}+\mathrm{B}), 1.42-1.29(\mathrm{~m}, 2 \mathrm{H}, \mathrm{A}+\mathrm{B}), 1.18-1.03(\mathrm{~m}, 4 \mathrm{H}$, A+B), $1.01-0.87(\mathrm{~m}, 1 \mathrm{H}, \mathrm{A}+\mathrm{B}), 0.82(\mathrm{td}, J=7.0,4.5,3 \mathrm{H}, \mathrm{A}+\mathrm{B}) . \underline{\text { See spectrum. }}$

${ }^{13} \mathbf{C ~ N M R}\left(100 \mathrm{MHz}, \mathrm{CDCl}_{3}\right) \delta_{\mathrm{C}}$ (mixture of diastereomers of major regioisomer reported) 179.2, 179.1, 179.0, 178.9, 143.9, 143.2, 141.5, 140.9, 136.4, 136.2, 133.5, 133.4, 132.3, 132.2, 129.4, 129.4, 128.9, $128.7,128.7,128.6,128.5,128.2,128.1,128.1,127.9,127.8,127.7,127.6,127.6,127.6,127.0,126.6$, 126.6, 126.3, 126.1, 126.1, 125.9, 125.8, 125.7, 125.2, 52.2, 52.1, 47.9, 47.8, 42.5, 42.4, 42.3, 40.0, $39.9,38.9,38.8,32.2,32.2,29.1,29.0,27.3,27.2,22.5,13.9$. See spectrum.

IR $v_{\max }: 2929,2858,1697,1389,1167,700 \mathrm{~cm}^{-1}$.

HRMS (ESI) m/z: [(M+Na) $\left.{ }^{+}\right]$calcd for $\mathrm{C}_{35} \mathrm{H}_{35} \mathrm{NO}_{2}$ 524.2560, found 524.2543.

$\left(1 R^{*}, 5 S^{*}, 7 R^{*}\right)$-3-Benzyl-7-butyl-1-(2-phenylpropyl)-3-azabicyclo[3.2.0]heptane-2,4-dione (17)

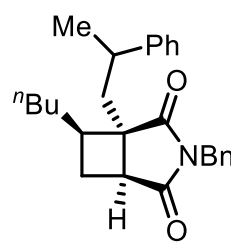

$52: 48$ d.r.

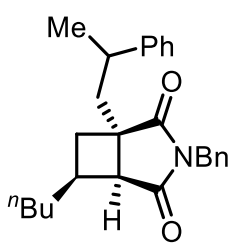

$10: 1$ r.r.

General procedure 5. Using chlorocyclobutane 4 ( $200 \mathrm{mg}, 0.65 \mathrm{mmol}, 10: 1$ r.r.), $\alpha$-methyl styrene (253 $\mu \mathrm{L}, 1.95 \mathrm{mmol})$, DIPEA (140 $\mu \mathrm{L}, 0.78 \mathrm{mmol})$, acetic acid (30 $\mu \mathrm{L}, 0.52 \mathrm{mmol}), 4 \mathrm{CzIPN}$ (26 mg, 5 $\mathrm{mol} \%$ ), and $\mathrm{MeCN} /$ water $(4: 1,2.0 \mathrm{~mL})$, with a reaction time of 6 hours. Purification by column chromatography (98/2 petroleum ether/ethyl acetate) gave the title compound (69 mg, 27\%) as a colourless oil.

${ }^{1} \mathbf{H}$ NMR $\left(500 \mathrm{MHz}, \mathrm{CDCl}_{3}\right) \delta_{\mathrm{H}}$ (mixture of diastereomers, $A+B$, of major regioisomer reported) 8.16 - 8.07 (m, 1H), $7.59-7.30(\mathrm{~m}, 4 \mathrm{H}), 7.26-6.63(\mathrm{~m}, 5 \mathrm{H}), 4.75-4.63(\mathrm{~m}, 2 \mathrm{H}, \mathrm{A}+\mathrm{B}), 2.99-2.77$ (m, $1 \mathrm{H}, \mathrm{A}+\mathrm{B}), 2.61-2.47(\mathrm{~m}, 1 \mathrm{H}, \mathrm{A}+\mathrm{B}), 2.45-2.10(\mathrm{~m}, 2 \mathrm{H}, \mathrm{A}+\mathrm{B}), 1.97-1.89(\mathrm{~m}, 1 \mathrm{H}, \mathrm{A}+\mathrm{B}), 1.83(\mathrm{dd}$, $J=10.5,5.0 \mathrm{~Hz}, 0.5 \mathrm{H}, \mathrm{A} / \mathrm{B}), 1.53-1.41(\mathrm{~m}, 1 \mathrm{H}, \mathrm{A}+\mathrm{B}), 1.39-1.00(\mathrm{~m}, 8.5 \mathrm{H}, \mathrm{A}+\mathrm{B}), 0.97-0.79(\mathrm{~m}$, $4 \mathrm{H}, \mathrm{A}+\mathrm{B})$. See spectrum. 
${ }^{13} \mathbf{C ~ N M R}\left(125 \mathrm{MHz}, \mathrm{CDCl}_{3}\right) \delta_{\mathrm{C}}$ (mixture of diastereomers of major regioisomer reported) $179.5,179.3$, 179.3, 179.0, 145.5, 145.4, 136.2, 136.1, 129.6, 128.8, 128.7, 128.6, 128.5, 128.5, 128.4, 128.1, 127.8, 127.2, 126.7, 126.6, 52.3, 51.7, 43.2, 42.7, 42.5, 42.4, 42.1, 42.1, 39.0, 38.8, 37.3, 37.1, 32.2, 32.1, 29.0, 29.0, 27.1, 27.0, 23.7, 23.4, 22.4, 22.4, 13.9, 13.8. See spectrum.

IR $v_{\max }: 2966,1700,1391,1055,700 \mathrm{~cm}^{-1}$.

HRMS (ESI) m/z: [(M+Na) $\left.{ }^{+}\right]$calcd for $\mathrm{C}_{26} \mathrm{H}_{31} \mathrm{NO}_{2}$ 412.2247, found 412.2235.

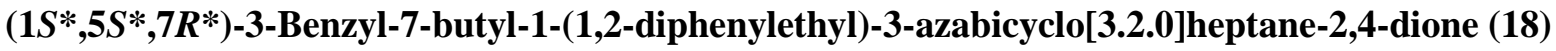
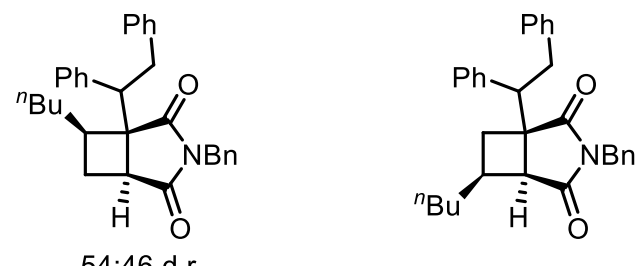

$15: 1$ r.r.

General procedure 5. Using chlorocyclobutane 4 (177 mg, $0.58 \mathrm{mmol}, 10: 1$ r.r.), trans-stilbene (348 $\mathrm{mg}, 1.95 \mathrm{mmol})$, DIPEA (140 $\mu \mathrm{L}, 0.78 \mathrm{mmol})$, acetic acid $(30 \mu \mathrm{L}, 0.52 \mathrm{mmol}), 4 \mathrm{CzIPN}(26 \mathrm{mg}, 5$ $\mathrm{mol} \%$ ), and $\mathrm{MeCN} /$ water $(4: 1,2.0 \mathrm{~mL})$, with a reaction time of 6 hours. Purification by column chromatography (98/2 petroleum ether/ethyl acetate) gave the title compound (143 $\mathrm{mg}, 55 \%$ ) as a colourless oil.

${ }^{1} \mathbf{H}$ NMR $\left(400 \mathrm{MHz}, \mathrm{CDCl}_{3}\right) \delta_{\mathrm{H}}$ (mixture of diastereomers, $A+B$, of major regioisomer reported) 7.28 - 6.77 (m, 15H, A+B), $4.57-4.40$ (m, 2H, A+B), $3.27-3.18$ (m, 1H, A+B), 3.12 (dd, $J=13.5,5.0$ $\mathrm{Hz}, 0.5 \mathrm{H}, \mathrm{A} / \mathrm{B}), 2.98$ (dd, $J=12.0,1.5 \mathrm{~Hz}, 0.5 \mathrm{H}, \mathrm{A} / \mathrm{B}), 2.94-2.82(\mathrm{~m}, 1 \mathrm{H}, \mathrm{A}+\mathrm{B}), 2.61-2.41$ (m, 1.5H, A+B), 2.29 (tdd, $J=10.0,7.0,5.0 \mathrm{~Hz}, 0.5 \mathrm{H}, \mathrm{A} / \mathrm{B}), 1.58-1.44(\mathrm{~m}, 1 \mathrm{H}, \mathrm{A}+\mathrm{B}), 1.44-1.34(\mathrm{~m}, 1 \mathrm{H}$, A+B), $1.31-0.62(\mathrm{~m}, 9 \mathrm{H}, \mathrm{A}+\mathrm{B}) . \underline{\text { See spectrum. }}$

${ }^{13} \mathbf{C ~ N M R}\left(100 \mathrm{MHz}, \mathrm{CDCl}_{3}\right) \delta_{\mathrm{C}}$ (mixture of diastereomers of major regioisomer reported) 179.2, 179.1, 178.2, 178.2, 139.9, 139.4, 139.2, 138.6, 135.9, 135.8, 129.0, 129.0, 128.9, 128.8, 128.8, 128.6, 128.6, $128.5,128.5,128.3,128.2,128.1,127.8,127.7,127.2,127.1,126.2,125.9,56.0,55.9,53.3,50.8,42.4$, $42.3,40.5,39.5,38.5,38.1,37.3,36.1,33.1,32.4,29.1,28.7,27.0,26.2,22.4,22.2,13.9,13.8$. See spectrum.

IR $v_{\max }: 2932,2855,1698,1495,1454,1389,1340,1171,1071,699 \mathrm{~cm}^{-1}$.

HRMS (ESI) m/z: [(M+Na) $)^{+}$calcd for $\mathrm{C}_{31} \mathrm{H}_{33} \mathrm{NO}_{2}$ 474.2404, found 474.2412. 
(19)

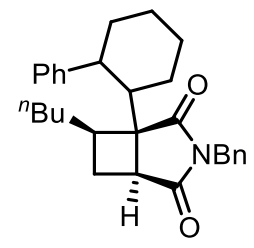

$61: 39$ d.r.

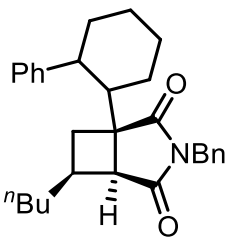

$9: 1$ r.r.

General procedure 5. Using chlorocyclobutane 4 (200 mg, $0.65 \mathrm{mmol}, 10: 1$ r.r.), 1-phenyl-1cyclohexene $(313 \mu \mathrm{L}, 1.95 \mathrm{mmol})$, DIPEA $(140 \mu \mathrm{L}, 0.78 \mathrm{mmol})$, acetic acid (30 $\mu \mathrm{L}, 0.52 \mathrm{mmol})$, 4CzIPN (26 mg, $5 \mathrm{~mol} \%$ ), and MeCN/water (4:1, $2.0 \mathrm{~mL}$ ), with a reaction time of 6 hours. Purification by column chromatography ( $98 / 2$ petroleum ether/ethyl acetate) gave the title compound ( $226 \mathrm{mg}, 45 \%$ ) as a colourless oil. For the major regioisomeric product, only two out of the four possible diastereomers were observed, however, their relative stereochemistry has not been determined.

${ }^{1} \mathbf{H}$ NMR $\left(400 \mathrm{MHz}, \mathrm{CDCl}_{3}\right) \delta_{\mathrm{H}}$ (mixture of diastereomers, $A+B$, of major regioisomer reported) 7.44 $-6.93(\mathrm{~m}, 10 \mathrm{H}), 4.46-4.26(\mathrm{~m}, 0.8 \mathrm{H}, \mathrm{B}), 4.18-3.92(\mathrm{~m}, 1.2 \mathrm{H}, \mathrm{A}), 3.09$ (s, 0.6H, A), 2.69 (dd, $J=$ 10.5, 5.0, 0.4H, B), $2.44-0.50(\mathrm{~m}, 22 \mathrm{H}, \mathrm{A}+\mathrm{B})$. See spectrum.

${ }^{13} \mathbf{C ~ N M R}\left(100 \mathrm{MHz}, \mathrm{CDCl}_{3}\right) \delta_{\mathrm{C}}$ (mixture of diastereomers of major regioisomer reported) 179.6, 179.1, $178.7,143.4,143.2$, 136.2, 135.9, 130.2, 129.8, 129.7, 129.4, 128.7, 128.6, 128.5, 128.4, 128.23, 128.20, 128.0, 127.9, 127.6, 126.7, 55.7, 55.1, 46.1, 42.22, 42.15, 41.8, 41.7, 41.1, 39.7, 38.0, 37.7, $36.9,33.1,32.8,31.9,29.0,27.0,26.6,26.5,26.1$, 23.9, 23.0, 22.9, 22.42, 22.39, 20.9, 20.8, 17.5, 14.7, 13.9, 13.8. See spectrum.

IR $v_{\max }: 2925,1694,1391,1055,701 \mathrm{~cm}^{-1}$.

HRMS (ESI) m/z: [(M+Na) $\left.{ }^{+}\right]$calcd for $\mathrm{C}_{26} \mathrm{H}_{31} \mathrm{NO}_{2} 412.2247$, found 412.2235. 

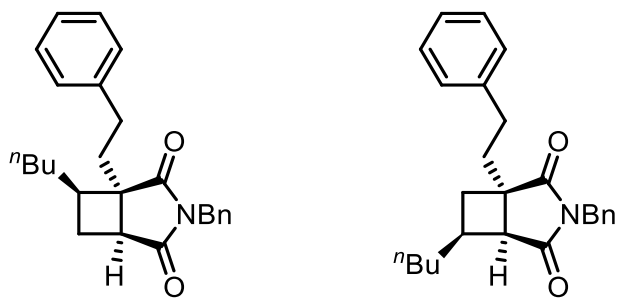

$10: 1$ r.r.

General procedure 5. Using chlorocyclobutane 4 (200 mg, $0.65 \mathrm{mmol}, 10: 1$ r.r.), styrene (223 $\mu \mathrm{L}, 1.95$ mmol), DIPEA (140 $\mu \mathrm{L}, 0.78 \mathrm{mmol})$, acetic acid ( $30 \mu \mathrm{L}, 0.52 \mathrm{mmol}), 4 \mathrm{CzIPN}$ (26 mg, $5 \mathrm{~mol} \%)$, and $\mathrm{MeCN} /$ water (4:1, $2.0 \mathrm{~mL})$, with a reaction time of 6 hours. Purification by column chromatography (98/2 petroleum ether/ethyl acetate) gave the title compound (105 mg, 43\%) as a colourless oil.

${ }^{1} \mathbf{H}$ NMR $\left(500 \mathrm{MHz}, \mathrm{CDCl}_{3}\right) \delta_{\mathrm{H}}$ (major regioisomer reported) $\left(500 \mathrm{MHz}, \mathrm{CDCl}_{3}\right) \delta 7.51-7.43(\mathrm{~m}$, $2 \mathrm{H}), 7.39-7.17(\mathrm{~m}, 6 \mathrm{H}), 7.07-7.03(\mathrm{~m}, 2 \mathrm{H}), 4.78-4.66(\mathrm{~m}, 2 \mathrm{H}), 2.75(\mathrm{dd}, J=10.5,5.0 \mathrm{~Hz}, 1 \mathrm{H})$, $2.64(\mathrm{dt}, J=12.5,10.0 \mathrm{~Hz}, 1 \mathrm{H}), 2.55-2.45(\mathrm{~m}, 2 \mathrm{H}), 2.40-2.29(\mathrm{~m}, 2 \mathrm{H}), 2.02-1.92(\mathrm{~m}, 1 \mathrm{H}), 1.57-$ $1.44(\mathrm{~m}, 2 \mathrm{H}), 1.23-1.14(\mathrm{~m}, 3 \mathrm{H}), 1.13-1.06(\mathrm{~m}, 1 \mathrm{H}), 1.02-0.94(\mathrm{~m}, 1 \mathrm{H}), 0.83(\mathrm{t}, J=7.0 \mathrm{~Hz}, 3 \mathrm{H})$. See spectrum.

${ }^{13} \mathbf{C}$ NMR $\left(125 \mathrm{MHz}, \mathrm{CDCl}_{3}\right) \delta_{\mathrm{C}}$ (major regioisomer reported) $178.6,178.4,140.0,135.4,128.2,127.9$, $127.8,127.5,127.2$, 125.5, 51.4, 41.7, 40.6, 38.3, 35.6, 31.6, 30.0, 28.3, 26.2, 21.7, 13.1. See spectrum. IR $v_{\max }: 3028,2927,2857,1766,1697,1495,1388,1339,1165,1069,747,698 \mathrm{~cm}^{-1}$.

HRMS (ESI) m/z: [(M+Na) $\left.{ }^{+}\right]$calcd for $\mathrm{C}_{25} \mathrm{H}_{29} \mathrm{NO}_{2}$ 398.2090, found 398.2103.

$\left(1 R^{*}, 5 S^{*}, 7 R^{*}\right)$-3-Benzyl-7-butyl-1-(4-methoxyphenethyl)-3-azabicyclo[3.2.0]heptane-2,4-dione (21)
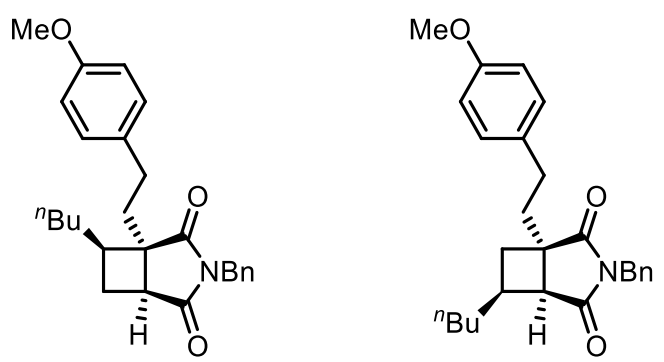

12:1 r.r.

General procedure 5. Using chlorocyclobutane 4 (200 mg, $0.65 \mathrm{mmol}, 10: 1$ r.r.), 4-vinylanisole (262 $\mu \mathrm{L}, 1.95 \mathrm{mmol})$, DIPEA (140 $\mu \mathrm{L}, 0.78 \mathrm{mmol})$, acetic acid (30 $\mu \mathrm{L}, 0.52 \mathrm{mmol}), 4 \mathrm{CzIPN}$ (26 mg, 5 $\mathrm{mol} \%$ ), and $\mathrm{MeCN} /$ water $(4: 1,2.0 \mathrm{~mL})$, with a reaction time of 6 hours. Purification by column 
chromatography (98/2 to $95 / 5$ petroleum ether/ethyl acetate) gave the title compound (30 $\mathrm{mg}, 15 \%)$ as a colourless oil.

${ }^{1} \mathbf{H}$ NMR $\left(500 \mathrm{MHz}, \mathrm{CDCl}_{3}\right) \delta_{\mathrm{H}}$ (major regioisomer reported) $7.50-7.25(\mathrm{~m}, 5 \mathrm{H}), 6.99-6.92(\mathrm{~m}, 2 \mathrm{H})$, $6.82-6.75(\mathrm{~m}, 2 \mathrm{H}), 4.77-4.65(\mathrm{~m}, 2 \mathrm{H}), 3.79(\mathrm{~s}, 3 \mathrm{H}), 2.75(\mathrm{dd}, J=10.5,5.0 \mathrm{~Hz}, 1 \mathrm{H}), 2.63(\mathrm{dt}, J=$ 13.0, $10.0 \mathrm{~Hz}, 1 \mathrm{H}), 2.46-2.40(\mathrm{~m}, 2 \mathrm{H}), 2.39-2.25(\mathrm{~m}, 2 \mathrm{H}), 1.92(\mathrm{ddd}, J=14.0,9.5,7.0 \mathrm{~Hz}, 1 \mathrm{H}), 1.58$ $-1.44(\mathrm{~m}, 2 \mathrm{H}), 1.32-1.04(\mathrm{~m}, 4 \mathrm{H}), 0.96(\mathrm{ddt}, J=15.1,11.0,5.5 \mathrm{~Hz}, 1 \mathrm{H}), 0.82$ (t, $J=7.0 \mathrm{~Hz}, 3 \mathrm{H}) . \underline{\text { See }}$ spectrum.

${ }^{13} \mathbf{C ~ N M R}\left(125 \mathrm{MHz}, \mathrm{CDCl}_{3}\right) \delta_{\mathrm{C}}$ (major regioisomer reported) 179.4, 179.2, 158.0, 136.2, 132.8, 129.1, 128.9, 128.6, 128.0, 113.9, 55.3, 52.2, 42.4, 41.3, 39.0, 36.6, 32.4, 29.9, 29.1, 26.9, 22.4, 13.9. See spectrum.

IR $v_{\text {max }}: 2929,2855,1764,1696,1511,1387,1245,1155,1033,699 \mathrm{~cm}^{-1}$.

HRMS (ESI) m/z: $\left[(\mathrm{M}+\mathrm{H})^{+}\right]$calcd for $\mathrm{C}_{26} \mathrm{H}_{32} \mathrm{NO}_{3} 406.2377$, found 406.2373 .

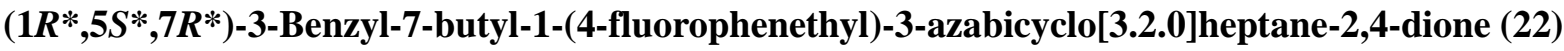
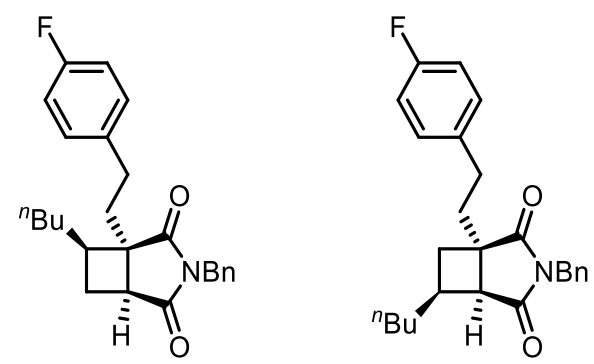

$18: 1$ r.r.

General procedure 5. Using chlorocyclobutane 4 (200 mg, $0.65 \mathrm{mmol}, 10: 1$ r.r.), 4-fluorostyrene (233 $\mu \mathrm{L}, 1.95 \mathrm{mmol})$, DIPEA (140 $\mu \mathrm{L}, 0.78 \mathrm{mmol})$, acetic acid $(30 \mu \mathrm{L}, 0.52 \mathrm{mmol}), 4 \mathrm{CzIPN}$ (26 mg, 5 $\mathrm{mol} \%$ ), and $\mathrm{MeCN} /$ water $(4: 1,2.0 \mathrm{~mL})$, with a reaction time of 6 hours. Purification by column chromatography (98/2 petroleum ether/ethyl acetate) gave the title compound (107 $\mathrm{mg}, 42 \%)$ as a colourless oil.

${ }^{1} \mathbf{H}$ NMR $\left(500 \mathrm{MHz}, \mathrm{CDCl}_{3}\right) \delta_{\mathrm{H}}$ (major regioisomer reported) $7.49-7.29(\mathrm{~m}, 5 \mathrm{H}), 7.03-6.89(\mathrm{~m}, 4 \mathrm{H})$, $4.76-4.64(\mathrm{~m}, 2 \mathrm{H}), 2.76(\mathrm{dd}, J=10.5,5.0 \mathrm{~Hz}, 1 \mathrm{H}), 2.65(\mathrm{dt}, J=13.0,10.0 \mathrm{~Hz}, 1 \mathrm{H}), 2.52-2.39$ (m, 2H), $2.39-2.23(\mathrm{~m}, 2 \mathrm{H}), 1.93(\mathrm{ddd}, J=14.0,10.0,6.5 \mathrm{~Hz}, 1 \mathrm{H}), 1.53(\mathrm{ddd}, J=12.5,7.5,5.0 \mathrm{~Hz}, 1 \mathrm{H})$, $1.49-1.43(\mathrm{~m}, 1 \mathrm{H}), 1.23-1.07(\mathrm{~m}, 4 \mathrm{H}), 1.01-0.92(\mathrm{~m}, 1 \mathrm{H}), 0.82(\mathrm{t}, J=6.9 \mathrm{~Hz}, 3 \mathrm{H})$. See spectrum. ${ }^{13} \mathrm{C}$ NMR $\left(125 \mathrm{MHz}, \mathrm{CDCl}_{3}\right) \delta_{\mathrm{C}}$ (major regioisomer reported) $179.3,179.1,161.4(\mathrm{~d}, J=244.0 \mathrm{~Hz})$, $136.4(\mathrm{~d}, J=3.0 \mathrm{~Hz}), 136.1,129.6(\mathrm{~d}, J=8.0 \mathrm{~Hz}), 129.0,128.6,128.0,115.3(\mathrm{~d}, J=21.0 \mathrm{~Hz}), 52.1$, 42.4, 41.3, 39.0, 36.5, 32.4, 30.0, 29.0, 26.9, 22.4, 13.9. See spectrum. 
IR $v_{\max }: 2928,2856,1738,1696,1508,1387,1342,1218,1157,1069,823,698 \mathrm{~cm}^{-1}$.

HRMS (ESI) m/z: [(M+Na) $]$ calcd for $\mathrm{C}_{25} \mathrm{H}_{28} \mathrm{FNO}_{2}$ 416.1996, found 416.2003.

Methyl 3-((1R*,5S*,7R*)-3-benzyl-7-butyl-2,4-dioxo-3-azabicyclo[3.2.0]heptan-1-yl)propanoate (23)
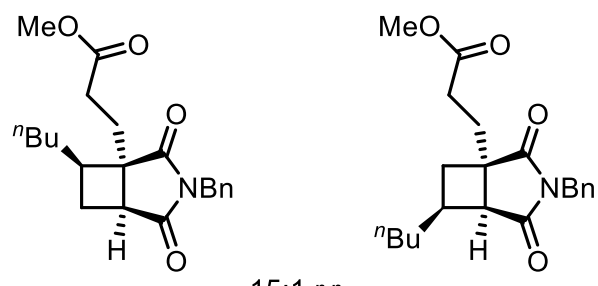

15:1 r.r.

General procedure 5. Using chlorocyclobutane 4 (200 mg, $0.65 \mathrm{mmol}, 10: 1$ r.r.), methyl acrylate (175 $\mu \mathrm{L}, 1.95 \mathrm{mmol})$, DIPEA (140 $\mu \mathrm{L}, 0.78 \mathrm{mmol})$, acetic acid (30 $\mu \mathrm{L}, 0.52 \mathrm{mmol}), 4 \mathrm{CzIPN}$ (26 mg, 5 $\mathrm{mol} \%$ ), and $\mathrm{MeCN} /$ water $(4: 1,2.0 \mathrm{~mL})$, with a reaction time of 6 hours. Purification by column chromatography (90/10 toluene/petroleum ether to remove the catalyst, followed by $95 / 5$ petroleum ether/ethyl acetate) gave the title compound (179 $\mathrm{mg}, 77 \%)$ as a yellow oil.

${ }^{1} \mathbf{H} \mathbf{N M R}\left(500 \mathrm{MHz}, \mathrm{CDCl}_{3}\right) \delta_{\mathrm{H}}$ (major regioisomer reported) $7.47-7.16(\mathrm{~m}, 5 \mathrm{H}), 4.77-4.62(\mathrm{~m}, 2 \mathrm{H})$, $3.64(\mathrm{~s}, 3 \mathrm{H}), 2.88(\mathrm{dd}, J=10.4,5.1 \mathrm{~Hz}, 1 \mathrm{H}), 2.71-2.57(\mathrm{~m}, 1 \mathrm{H}), 2.41-2.20(\mathrm{~m}, 4 \mathrm{H}), 2.12-1.96(\mathrm{~m}$, $1 \mathrm{H}), 1.53(\mathrm{ddd}, J=12.5,7.5,5.0 \mathrm{~Hz}, 1 \mathrm{H}), 1.45$ (ddt, $J=13.5,11.0,5.5 \mathrm{~Hz}, 1 \mathrm{H}), 1.26-1.02(\mathrm{~m}, 4 \mathrm{H})$, $1.01-0.86(\mathrm{~m}, 1 \mathrm{H}), 0.82(\mathrm{t}, J=7.0 \mathrm{~Hz}, 3 \mathrm{H})$. See spectrum.

${ }^{13} \mathbf{C ~ N M R}\left(125 \mathrm{MHz}, \mathrm{CDCl}_{3}\right) \delta_{\mathrm{C}}$ (major regioisomer reported) 179.1, 178.8, 173.1, 136.1, 128.8, 128.6, 127.9, 51.8, 51.2, 42.4, 40.9, 39.1, 32.4, 29.5, 29.2, 29.0, 26.7, 22.4, 13.9. See spectrum.

IR $v_{\max }: 2929,2857,1737,1696,1496,1434,1388,1166,1070,731,699 \mathrm{~cm}^{-1}$.

HRMS (ESI) m/z: [(M+Na) $\left.{ }^{+}\right]$calcd for $\mathrm{C}_{21} \mathrm{H}_{27} \mathrm{NO}_{4} 380.1832$, found 380.1836 . 


\section{Ethyl 3-((1 $\left.R^{*}, 5 S^{*}, 7 R^{*}\right)-3$-benzyl-7-butyl-2,4-dioxo-3-azabicyclo[3.2.0]heptan-1-yl)propanoate}

(24)

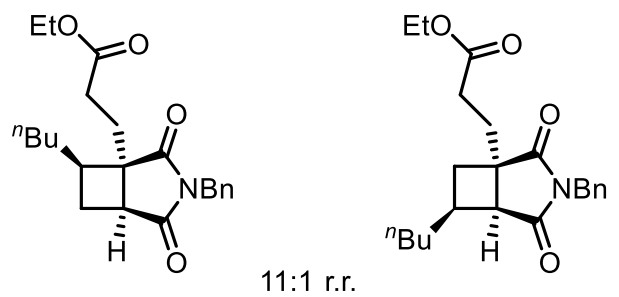

General procedure 5. Using chlorocyclobutane $4(200 \mathrm{mg}, 0.65 \mathrm{mmol}, 10: 1 \mathrm{r} . \mathrm{r}$.), ethyl acrylate $(212 \mu \mathrm{L}$, $1.95 \mathrm{mmol})$, DIPEA ( $140 \mu \mathrm{L}, 0.78 \mathrm{mmol})$, acetic acid ( $30 \mu \mathrm{L}, 0.52 \mathrm{mmol}), 4 \mathrm{CzIPN}(26 \mathrm{mg}, 5 \mathrm{~mol} \%)$, and $\mathrm{MeCN} /$ water $(4: 1,2.0 \mathrm{~mL})$, with a reaction time of 6 hours. Purification by column chromatography ( $90 / 10$ toluene/petroleum ether to remove the catalyst, followed by $95 / 5$ petroleum ether/ethyl acetate) gave the title compound (181 mg, 75\%) as a yellow oil.

${ }^{1} \mathbf{H} \mathbf{N M R}\left(400 \mathrm{MHz}, \mathrm{CDCl}_{3}\right) \delta_{\mathrm{H}}$ (major regioisomer reported) $7.35-7.30(\mathrm{~m}, 2 \mathrm{H}), 7.25-7.19(\mathrm{~m}, 3 \mathrm{H})$, $4.63-4.58(\mathrm{~m}, 2 \mathrm{H}), 4.06-3.98(\mathrm{~m}, 2 \mathrm{H}), 2.80(\mathrm{dd}, J=10.5,5.0 \mathrm{~Hz}, 1 \mathrm{H}), 2.62-2.53(\mathrm{~m}, 1 \mathrm{H}), 2.31-$ $2.22(\mathrm{~m}, 1 \mathrm{H}), 2.23-2.09(\mathrm{~m}, 3 \mathrm{H}), 2.01-1.90(\mathrm{~m}, 1 \mathrm{H}), 1.47-1.40(\mathrm{~m}, 1 \mathrm{H}), 1.40-1.30(\mathrm{~m}, 1 \mathrm{H}), 1.15$ (t, $J=7.0 \mathrm{~Hz}, 3 \mathrm{H}), 1.11-1.00(\mathrm{~m}, 4 \mathrm{H}), 0.85-0.78(\mathrm{~m}, 1 \mathrm{H}), 0.72(\mathrm{t}, J=7.0 \mathrm{~Hz}, 3 \mathrm{H}) . \underline{\text { See spectrum. }}$

${ }^{13} \mathbf{C ~ N M R}\left(100 \mathrm{MHz}, \mathrm{CDCl}_{3}\right) \delta_{\mathrm{C}}$ (major regioisomer reported) $179.1,178.8,172.7,136.1,128.7,128.6$, 127.9, 60.7, 51.2, 42.4, 40.9, 39.1, 32.4, 29.5, 29.4, 29.0, 26.7, 22.4, 14.1, 13.9. See spectrum.

IR $v_{\max }: 2930,2858,1732,1698,1455,1389,1342,1166,1071,701 \mathrm{~cm}^{-1}$.

HRMS (ESI) m/z: [(M+H) $]$ calcd for $\mathrm{C}_{22} \mathrm{H}_{28} \mathrm{NO}_{4} 372.2169$, found 372.2173.

\section{Benzyl 3-((1R*,5 $\left.S^{*}, 7 R^{*}\right)-3$-benzyl-7-butyl-2,4-dioxo-3-azabicyclo[3.2.0]heptan-1-yl)propanoate} (25)

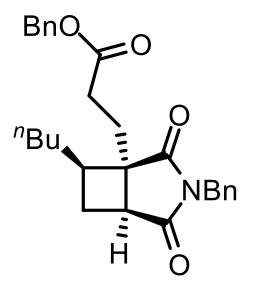

13:1 r.r.

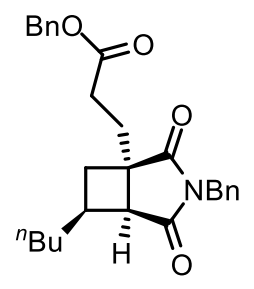

General procedure 5. Using chlorocyclobutane 4 (200 mg, $0.65 \mathrm{mmol}, 10: 1$ r.r.), benzyl acrylate (296 $\mu \mathrm{L}, 1.95 \mathrm{mmol})$, DIPEA (140 $\mu \mathrm{L}, 0.78 \mathrm{mmol})$, acetic acid $(30 \mu \mathrm{L}, 0.52 \mathrm{mmol}), 4 \mathrm{CzIPN}$ (26 mg, 5 $\mathrm{mol} \%$ ), and $\mathrm{MeCN} /$ water $(4: 1,2.0 \mathrm{~mL})$, with a reaction time of 6 hours. Purification by column chromatography (90/10 toluene/petroleum ether to remove the catalyst, followed by $95 / 5$ petroleum ether/ethyl acetate) gave the title compound (127 mg, 45\%) as a yellow oil. 
${ }^{1} \mathbf{H}$ NMR $\left(500 \mathrm{MHz}, \mathrm{CDCl}_{3}\right) \delta_{\mathrm{H}}$ (major regioisomer reported) $7.42-7.29(\mathrm{~m}, 10 \mathrm{H}), 5.14-5.04(\mathrm{~m}$, 2H), $4.74-4.62(\mathrm{~m}, 2 \mathrm{H}), 2.88(\mathrm{dd}, J=10.5,5.0 \mathrm{~Hz}, 1 \mathrm{H}), 2.68-2.63(\mathrm{~m}, 2 \mathrm{H}), 2.42-2.22(\mathrm{~m}, 3 \mathrm{H})$, $2.10-2.02(\mathrm{~m}, 1 \mathrm{H}), 1.52(\mathrm{ddd}, J=12.5,7.5,5.0 \mathrm{~Hz}, 1 \mathrm{H}), 1.48-1.40(\mathrm{~m}, 1 \mathrm{H}), 1.22-1.05(\mathrm{~m}, 4 \mathrm{H})$, $0.94-0.87(\mathrm{~m}, 1 \mathrm{H}), 0.81(\mathrm{t}, J=7.0 \mathrm{~Hz}, 3 \mathrm{H})$. See spectrum.

${ }^{13} \mathbf{C}$ NMR $\left(125 \mathrm{MHz}, \mathrm{CDCl}_{3}\right) \delta_{\mathrm{C}}$ (major regioisomer reported) 179.0, 178.8, 172.4, 136.1, 135.7, 128.7, 128.6, 128.6, 128.3, 128.2, 127.9, 66.5, 51.1, 42.4, 40.8, 39.1, 29.4, 29.3, 29.0, 28.0, 26.7, 22.4, 13.9 . See spectrum.

IR $v_{\max }: 2929,2857,1736,1697,1455,1386,1351,1156,735,697 \mathrm{~cm}^{-1}$.

HRMS (ESI) m/z: [(M+Na) $)^{+}$calcd for $\mathrm{C}_{27} \mathrm{H}_{31} \mathrm{NO}_{4} 456.2145$, found 456.2157.

\section{Butyl 3-((1R*,5S*,7 $\left.R^{*}\right)$-3-benzyl-7-butyl-2,4-dioxo-3-azabicyclo[3.2.0]heptan-1-yl)propanoate} (26)
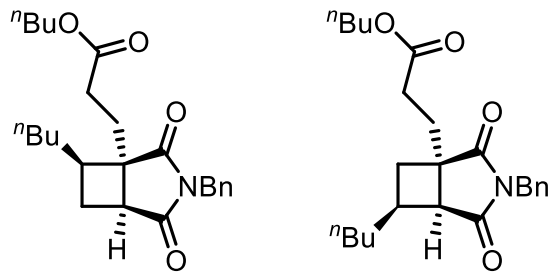

$8: 1$ r.r.

General procedure 5. Using chlorocyclobutane 4 (200 mg, $0.65 \mathrm{mmol}, 10: 1$ r.r.), $n$-butyl acrylate (282 $\mu \mathrm{L}, 1.95 \mathrm{mmol})$, DIPEA (140 $\mu \mathrm{L}, 0.78 \mathrm{mmol})$, acetic acid $(30 \mu \mathrm{L}, 0.52 \mathrm{mmol}), 4 \mathrm{CzIPN}$ (26 mg, 5 $\mathrm{mol} \%$ ), and $\mathrm{MeCN} /$ water $(4: 1,2.0 \mathrm{~mL})$, with a reaction time of 6 hours. Purification by column chromatography (90/10 toluene/petroleum ether to remove the catalyst, followed by $95 / 5$ petroleum ether/ethyl acetate) gave the title compound (203 mg, 78\%) as a colourless oil.

${ }^{1} \mathbf{H} \mathbf{N M R}\left(400 \mathrm{MHz}, \mathrm{CDCl}_{3}\right) \delta_{\mathrm{H}}$ (major regioisomer reported) $7.47-7.23(\mathrm{~m}, 5 \mathrm{H}), 4.76-4.63(\mathrm{~m}, 2 \mathrm{H})$, $4.16-3.99(\mathrm{~m}, 2 \mathrm{H}), 2.89(\mathrm{dd}, J=10.5,5.0 \mathrm{~Hz}, 1 \mathrm{H}), 2.73-2.57(\mathrm{~m}, 1 \mathrm{H}), 2.41-2.29(\mathrm{~m}, 1 \mathrm{H}), 2.29-$ $2.19(\mathrm{~m}, 3 \mathrm{H}), 2.10-1.99(\mathrm{~m}, 1 \mathrm{H}), 1.67-1.48(\mathrm{~m}, 2 \mathrm{H}), 1.48-1.26(\mathrm{~m}, 4 \mathrm{H}), 1.24-1.01(\mathrm{~m}, 4 \mathrm{H}), 0.99$ -0.92 (m, 4H), $0.85-0.79$ (m, 3H). See spectrum.

${ }^{13} \mathbf{C} \mathbf{N M R}\left(100 \mathrm{MHz}, \mathrm{CDCl}_{3}\right) \delta_{\mathrm{C}}$ (major regioisomer reported) $179.1,178.9,172.7,136.1,128.8,128.6$, 127.9, 64.6, 51.2, 42.4, 40.9, 39.1, 32.4, 30.6, 29.5, 29.3, 29.0, 26.7, 22.4, 19.1, 13.9, 13.7. See spectrum. IR $v_{\max }: 2958,2931,2877,1732,1699,1389,1342,1166,1070,700 \mathrm{~cm}^{-1}$.

HRMS (ESI) m/z: [(M+H)+] calcd for $\mathrm{C}_{24} \mathrm{H}_{33} \mathrm{NO}_{4} 400.2482$, found 400.2471 . 
tert-Butyl 3-((1R*,5S*,7R*)-3-benzyl-7-butyl-2,4-dioxo-3-azabicyclo[3.2.0]heptan-1-

yl)propanoate (27)
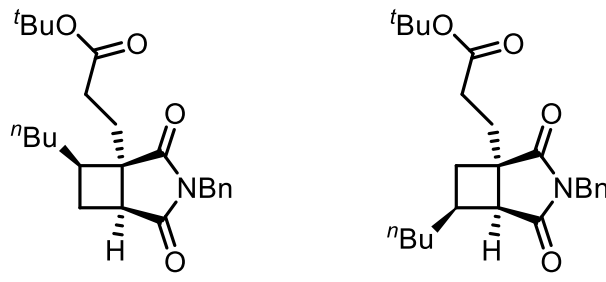

$12: 1$ r.r.

General procedure 5. Using chlorocyclobutane 4 (200 mg, $0.65 \mathrm{mmol}, 10: 1$ r.r.), $t$-butyl acrylate (284 $\mu \mathrm{L}, 1.95 \mathrm{mmol})$, DIPEA (140 $\mu \mathrm{L}, 0.78 \mathrm{mmol})$, acetic acid $(30 \mu \mathrm{L}, 0.52 \mathrm{mmol}), 4 \mathrm{CzIPN}(26 \mathrm{mg}, 5$ $\mathrm{mol} \%$ ), and $\mathrm{MeCN} /$ water $(4: 1,2.0 \mathrm{~mL})$, with a reaction time of 6 hours. Purification by column chromatography (90/10 toluene/petroleum ether to remove the catalyst, followed by $95 / 5$ petroleum ether/ethyl acetate) gave the title compound $(207 \mathrm{mg}, 80 \%)$ as a yellow oil.

${ }^{1} \mathbf{H}$ NMR $\left(400 \mathrm{MHz}, \mathrm{CDCl}_{3}\right) \delta_{\mathrm{H}}$ (major regioisomer reported) $7.37-7.16(\mathrm{~m}, 5 \mathrm{H}), 4.65-4.53(\mathrm{~m}, 2 \mathrm{H})$, $2.82(\mathrm{dd}, J=10.5,5.0 \mathrm{~Hz}, 1 \mathrm{H}), 2.63-2.51(\mathrm{~m}, 1 \mathrm{H}), 2.33-2.22(\mathrm{~m}, 1 \mathrm{H}), 2.15-2.01(\mathrm{~m}, 3 \mathrm{H}), 1.98-$ $1.85(\mathrm{~m}, 1 \mathrm{H}), 1.51-1.35(\mathrm{~m}, 2 \mathrm{H}), 1.34(\mathrm{~s}, 9 \mathrm{H}), 1.19-0.96(\mathrm{~m}, 4 \mathrm{H}), 0.91-0.77(\mathrm{~m}, 1 \mathrm{H}), 0.77-0.69$ $(\mathrm{m}, 3 \mathrm{H})$. See spectrum.

${ }^{13} \mathbf{C}$ NMR $\left(100 \mathrm{MHz}, \mathrm{CDCl}_{3}\right) \delta_{\mathrm{C}}$ (major regioisomer reported) $179.1,179.0,171.9,136.1,128.7,128.6$, 127.9, 80.8, 51.3, 42.4, 40.9, 39.1, 32.4, 30.5, 29.5, 29.0, 28.0, 26.8, 22.4, 13.9. See spectrum.

IR $v_{\max }: 2970,2930,2858,1726,1698,1496,1455,1366,1149,1070,699 \mathrm{~cm}^{-1}$.

HRMS (ESI) m/z: $\left[(\mathrm{M}+\mathrm{Na})^{+}\right]$calcd for $\mathrm{C}_{24} \mathrm{H}_{33} \mathrm{NO}_{4} 422.2295$, found 422.2302 .

\section{3-((1R*,5S*,7R*)-3-Benzyl-7-butyl-2,4-dioxo-3-azabicyclo[3.2.0]heptan-1-yl)- $N, N$ -}

\section{dimethylpropanamide (28)}
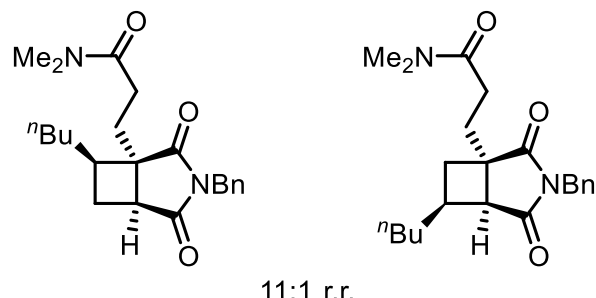

General procedure 5. Using chlorocyclobutane 4 (200 mg, $0.65 \mathrm{mmol}, 10: 1$ r.r.), N,N-dimethyl acrylamide (201 $\mu \mathrm{L}, 1.95 \mathrm{mmol})$, DIPEA (140 $\mu \mathrm{L}, 0.78 \mathrm{mmol})$, acetic acid (30 $\mu \mathrm{L}, 0.52 \mathrm{mmol}), 4 \mathrm{CzIPN}$ (26 mg, $5 \mathrm{~mol} \%)$, and MeCN/water $(4: 1,2.0 \mathrm{~mL})$, with a reaction time of 6 hours. Purification by 
column chromatography (90/10 toluene/petroleum ether to remove the catalyst, followed by $90 / 10$ to $50 / 50$ petroleum ether/ethyl acetate) gave the title compound (156 mg, 65\%) as a colourless oil.

${ }^{1} \mathbf{H}$ NMR $\left(400 \mathrm{MHz}, \mathrm{CDCl}_{3}\right) \delta_{\mathrm{H}}$ (major regioisomer reported) $7.43-7.21(\mathrm{~m}, 5 \mathrm{H}), 4.68(\mathrm{~s}, 2 \mathrm{H}), 2.94$ $-2.84(\mathrm{~m}, 1 \mathrm{H}), 2.88(\mathrm{~s}, 3 \mathrm{H}), 2.78(\mathrm{~s}, 3 \mathrm{H}), 2.66(\mathrm{dt}, J=13.0,10.0 \mathrm{~Hz}, 1 \mathrm{H}), 2.36(\mathrm{tt}, J=9.5,7.0 \mathrm{~Hz}, 1 \mathrm{H})$, $2.28-2.16(\mathrm{~m}, 1 \mathrm{H}), 2.16-2.01(\mathrm{~m}, 3 \mathrm{H}), 1.52(\mathrm{ddd}, J=12.5,7.5,5.0 \mathrm{~Hz}, 1 \mathrm{H}), 1.48-1.39(\mathrm{~m}, 1 \mathrm{H})$, $1.22-1.06(\mathrm{~m}, 4 \mathrm{H}), 1.01-0.86(\mathrm{~m}, 1 \mathrm{H}), 0.83-0.77(\mathrm{~m}, 3 \mathrm{H}) . \underline{\text { See spectrum. }}$

${ }^{13} \mathbf{C ~ N M R}\left(100 \mathrm{MHz}, \mathrm{CDCl}_{3}\right) \delta_{\mathrm{C}}$ (major regioisomer reported) $179.4,179.3,171.5,136.3,128.8,128.6$, 127.9, 51.5, 42.4, 41.0, 39.2, 37.0, 35.5, 32.5, 29.9, 29.0, 27.9, 26.8, 22.4, 13.9. See spectrum.

IR $v_{\max }: 3454,2929,2852,1738,1694,1643,1496,1455,1387,1345,1216,1070,699 \mathrm{~cm}^{-1}$.

HRMS HRMS (ESI) m/z: [(M+Na) $\left.{ }^{+}\right]$calcd for $\mathrm{C}_{22} \mathrm{H}_{30} \mathrm{~N}_{2} \mathrm{O}_{3} 393.2149$, found 393.2156.

3-((1R*,5S*,7R*)-3-Benzyl-7-butyl-2,4-dioxo-3-azabicyclo[3.2.0]heptan-1-yl)propanenitrile (29)

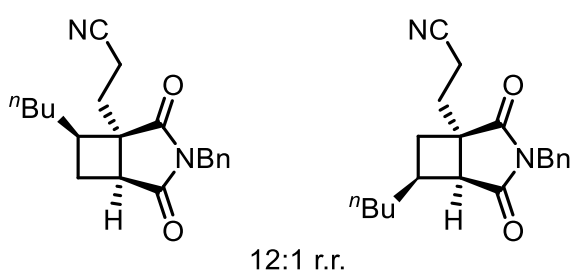

General procedure 5. Using chlorocyclobutane 4 (200 mg, $0.65 \mathrm{mmol}, 10: 1$ r.r.), acrylonitrile (128 $\mu \mathrm{L}$, $1.95 \mathrm{mmol})$, DIPEA ( $140 \mu \mathrm{L}, 0.78 \mathrm{mmol})$, acetic acid ( $30 \mu \mathrm{L}, 0.52 \mathrm{mmol}), 4 \mathrm{CzIPN}(26 \mathrm{mg}, 5 \mathrm{~mol} \%)$, and $\mathrm{MeCN} /$ water $(4: 1,2.0 \mathrm{~mL}$ ), with a reaction time of 6 hours. Purification by column chromatography ( $90 / 10$ toluene/petroleum ether to remove the catalyst, followed by $80 / 20$ petroleum ether/ethyl acetate) gave the title compound (148 $\mathrm{mg}, 70 \%)$ as a colourless oil.

${ }^{1} \mathbf{H} \mathbf{N M R}\left(500 \mathrm{MHz}, \mathrm{CDCl}_{3}\right) \delta_{\mathrm{H}}($ major regioisomer reported) $7.46-7.24(\mathrm{~m}, 5 \mathrm{H}), 4.70(\mathrm{~s}, 2 \mathrm{H}), 3.03$ $(\mathrm{dd}, J=10.5,5.0 \mathrm{~Hz}, 1 \mathrm{H}), 2.73(\mathrm{dt}, J=13.0,10.0 \mathrm{~Hz}, 1 \mathrm{H}), 2.47-2.31(\mathrm{~m}, 3 \mathrm{H}), 2.27(\mathrm{ddd}, J=14.5$, 9.0, $6.0 \mathrm{~Hz}, 1 \mathrm{H}), 2.10-2.01(\mathrm{~m}, 1 \mathrm{H}), 1.62-1.54(\mathrm{~m}, 1 \mathrm{H}), 1.49-1.39(\mathrm{~m}, 1 \mathrm{H}), 1.22-1.05(\mathrm{~m}, 4 \mathrm{H})$, $0.97-0.87(\mathrm{~m}, 1 \mathrm{H}), 0.81(\mathrm{t}, J=7.0 \mathrm{~Hz}, 3 \mathrm{H})$. See spectrum.

${ }^{13} \mathbf{C}$ NMR $\left(125 \mathrm{MHz}, \mathrm{CDCl}_{3}\right) \delta_{\mathrm{C}}$ (major regioisomer reported) $178.3,178.2,135.8,128.8,128.7,128.1$, $118.7,50.5,42.6,40.7,39.1,32.4,30.0,28.8,26.7,22.4,13.8,12.7$. See spectrum.

IR $v_{\max }: 3065,2956,2934,2239,1693,1426,1390,1361,1337,1169,937,698 \mathrm{~cm}^{-1}$.

HRMS (ESI) m/z: [(M+Na) $)^{+}$calcd for $\mathrm{C}_{20} \mathrm{H}_{24} \mathrm{~N}_{2} \mathrm{O}_{2} 347.1730$, found 347.1735. 


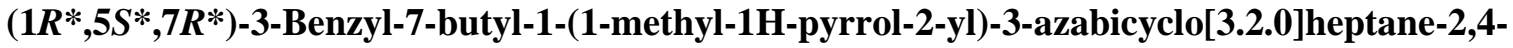
dione (30)

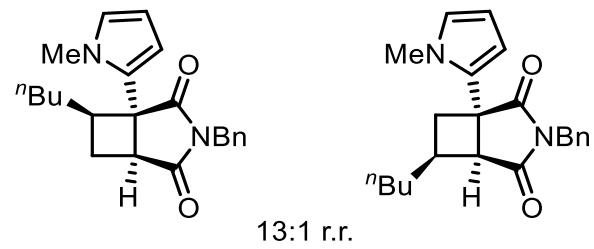

General procedure 5. Using chlorocyclobutane 4 (200 mg, $0.65 \mathrm{mmol}, 10: 1$ r.r.), $N$-methylpyrrole (580 $\mu \mathrm{L}, 6.50 \mathrm{mmol})$, DIPEA (140 $\mu \mathrm{L}, 0.78 \mathrm{mmol})$, acetic acid $(30 \mu \mathrm{L}, 0.52 \mathrm{mmol}), 4 \mathrm{CzIPN}$ (26 mg, 5 $\mathrm{mol} \%$ ), and $\mathrm{MeCN} /$ water $(4: 1,2.0 \mathrm{~mL})$, with a reaction time of 6 hours. Purification by column chromatography (98/2 to 95/5 petroleum ether/ethyl acetate) gave the title compound (112 $\mathrm{mg}, 49 \%)$ as a yellow oil.

${ }^{1} \mathbf{H}$ NMR $\left(400 \mathrm{MHz}, \mathrm{CDCl}_{3}\right) \delta_{\mathrm{H}}$ (major regioisomer reported) $7.40-7.31(\mathrm{~m}, 2 \mathrm{H}), 7.29-7.16(\mathrm{~m}, 3 \mathrm{H})$, $6.49(\mathrm{dd}, J=2.5,2.0 \mathrm{~Hz}, 1 \mathrm{H}), 6.03-5.91(\mathrm{~m}, 2 \mathrm{H}), 4.71-4.57(\mathrm{~m}, 2 \mathrm{H}), 3.34(\mathrm{~s}, 3 \mathrm{H}), 3.26(\mathrm{ddd}, J=$ 10.5, 4.5, $0.5 \mathrm{~Hz}, 1 \mathrm{H}), 2.92-2.77(\mathrm{~m}, 1 \mathrm{H}), 2.69(\mathrm{dt}, J=13.0,10.0 \mathrm{~Hz}, 1 \mathrm{H}), 1.71-1.56(\mathrm{~m}, 2 \mathrm{H}), 1.27$ $-0.90(\mathrm{~m}, 5 \mathrm{H}), 0.78-0.70(\mathrm{~m}, 3 \mathrm{H})$. See spectrum.

${ }^{13} \mathbf{C}$ NMR $\left(100 \mathrm{MHz}, \mathrm{CDCl}_{3}\right) \delta_{\mathrm{C}}$ (major regioisomer reported) $178.8,176.7,135.8,129.1,129.0,128.6$, 128.1, 124.7, 108.1, 106.9, 50.4, 42.7, 41.0, 40.8, 35.0, 32.6, 28.7, 26.7, 22.3, 13.9. See spectrum.

IR $v_{\max }: 2955,2917,2850,1769,1700,1466,1387,1172,1072 \mathrm{~cm}^{-1}$.

HRMS (ESI) m/z: [(M+H) $\left.{ }^{+}\right]$calcd for $\mathrm{C}_{22} \mathrm{H}_{26} \mathrm{~N}_{2} \mathrm{O}_{2}$ 351.2067, found 351.2064.

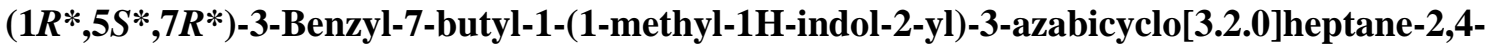
dione (31)
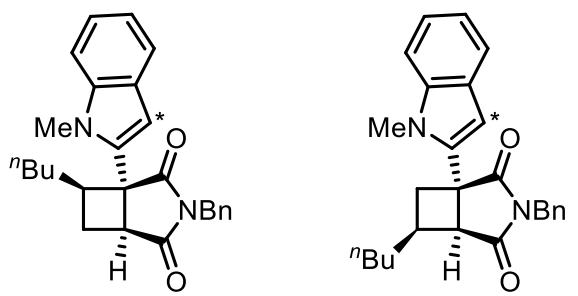

60:40 C2:C3

$20: 1$ r.r.

General procedure 5. Using chlorocyclobutane 4 (200 mg, $0.65 \mathrm{mmol}, 10: 1$ r.r.), $N$-methylindole (812 $\mu \mathrm{L}, 6.50 \mathrm{mmol})$, DIPEA (140 $\mu \mathrm{L}, 0.78 \mathrm{mmol})$, acetic acid $(30 \mu \mathrm{L}, 0.52 \mathrm{mmol}), 4 \mathrm{CzIPN}(26 \mathrm{mg}, 5$ $\mathrm{mol} \%$ ), and $\mathrm{MeCN} /$ water $(4: 1,2.0 \mathrm{~mL})$, with a reaction time of 6 hours. Purification by column chromatography ( $95 / 5$ to $90 / 10$ petroleum ether/ethyl acetate) gave the title compound ( $52 \mathrm{mg}, 20 \%$ ) as an orange oil. 
${ }^{1} \mathbf{H}$ NMR $\left(400 \mathrm{MHz}, \mathrm{CDCl}_{3}\right) \delta_{\mathrm{H}}$ (mixture of indole isomers, $A+B$, of major regioisomer reported) 7.40 $-6.84(\mathrm{~m}, 9.6 \mathrm{H}, \mathrm{A}+\mathrm{B}), 5.87(\mathrm{dd}, J=3.2,0.8 \mathrm{~Hz}, 0.4 \mathrm{H}, \mathrm{B}), 4.79-4.54(\mathrm{~m}, 2 \mathrm{H}, \mathrm{A}+\mathrm{B}), 3.66(\mathrm{~s}, 1.8 \mathrm{H}$, A), $3.65(\mathrm{~s}, 1.2 \mathrm{H}, \mathrm{B}), 3.56(\mathrm{dd}, J=10.5,5.2 \mathrm{~Hz}, 0.6 \mathrm{H}, \mathrm{A}), 3.51-3.36(\mathrm{~m}, 0.4 \mathrm{H}, \mathrm{B}) 3.26-3.11$ (m, $0.6 \mathrm{H}, \mathrm{A}), 3.03-2.92(\mathrm{~m}, 0.4 \mathrm{H}, \mathrm{B}), 2.88-2.58(\mathrm{~m}, 1 \mathrm{H}, \mathrm{A}+\mathrm{B}), 1.90-1.78(\mathrm{~m}, 0.6 \mathrm{H}, \mathrm{A}), 1.70-1.55$ $(\mathrm{m}, 1.4 \mathrm{H}, \mathrm{A}+\mathrm{B}), 1.27-1.03(\mathrm{~m}, 5 \mathrm{H}, \mathrm{A}+\mathrm{B}), 0.88-0.69(\mathrm{~m}, 3 \mathrm{H}, \mathrm{A}+\mathrm{B})$. See spectrum.

${ }^{13} \mathbf{C}$ NMR (100 MHz, $\left.\mathrm{CDCl}_{3}\right) \delta_{\mathrm{C}}$ (mixture of indole isomers of major regioisomer reported) 179.8 , 179.4, 178.1, 177.7, 137.6, 137.1, 136.1, 136.0, 130.6, 129.0, 129.0, 128.9, 128.9, 128.6, 128.6, 127.9, $127.9,126.6,125.8,125.5,122.1,121.5,119.5,119.2,117.5,109.8,109.2,99.1,55.8,50.9,42.8,42.6$, $42.2,41.9,41.8,40.7,33.2,33.0,32.8,32.8,29.0,29.0,27.5,22.5,22.4,14.0,13.9,13.9$. See spectrum. IR $v_{\max }: 2930,2856,1738,1698,1455,1384,1343,1216,745,699 \mathrm{~cm}^{-1}$.

HRMS (ESI) m/z: [(M+Na) $\left.{ }^{+}\right]$calcd for $\mathrm{C}_{26} \mathrm{H}_{28} \mathrm{~N}_{2} \mathrm{O}_{2}$ 423.2043, found 423.2051.

$\left(1 R^{*}, 5 S^{*}, 7 R^{*}\right)$-3-Benzyl-7-butyl-1-(2-oxo-2-phenylethyl)-3-azabicyclo[3.2.0]heptane-2,4-dione (32)
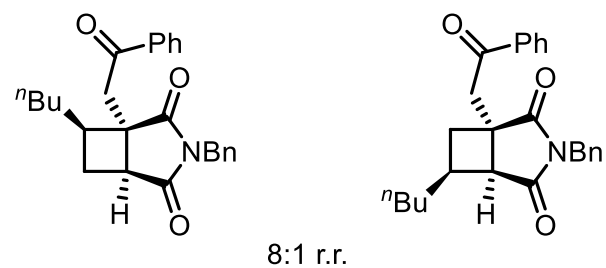

General procedure 5. Using chlorocyclobutane 4 (200 mg, $0.65 \mathrm{mmol}, 10: 1$ r.r.), trimethyl((1phenylvinyl)oxy)silane ( $\mathbf{S 4}, 375 \mathrm{mg}, 1.95 \mathrm{mmol})$, DIPEA $(140 \mu \mathrm{L}, 0.78 \mathrm{mmol})$, acetic acid $(30 \mu \mathrm{L}$, $0.52 \mathrm{mmol}$ ), 4CzIPN (26 mg, $5 \mathrm{~mol} \%$ ), and MeCN/water (4:1, $2.0 \mathrm{~mL}$ ), with a reaction time of 6 hours. Purification by column chromatography ( $90 / 10$ toluene/petroleum ether to remove the catalyst, then 95/5 petroleum ether/ethyl acetate) gave the title compound (51 mg, 20\%) as a colourless oil.

${ }^{1} \mathbf{H}$ NMR $\left(400 \mathrm{MHz}, \mathrm{CDCl}_{3}\right) \delta_{\mathrm{H}}$ (major regioisomer reported) $7.90-7.77(\mathrm{~m}, 2 \mathrm{H}), 7.54-7.45(\mathrm{~m}, 1 \mathrm{H})$, $7.41-7.36$ (m, 3H), $7.27-7.14(\mathrm{~m}, 4 \mathrm{H}), 4.80-4.62(\mathrm{~m}, 2 \mathrm{H}), 3.78$ (d, $J=18.5 \mathrm{~Hz}, 1 \mathrm{H}), 3.34$ (d, $J=$ $18.5 \mathrm{~Hz}, 1 \mathrm{H}), 2.98(\mathrm{dd}, J=10.5,5.0 \mathrm{~Hz}, 1 \mathrm{H}), 2.65(\mathrm{dt}, J=13.0,10.0 \mathrm{~Hz}, 1 \mathrm{H}), 2.39-2.28(\mathrm{~m}, 1 \mathrm{H})$, $1.58-1.52(\mathrm{~m}, 1 \mathrm{H}), 1.42-1.32(\mathrm{~m}, 1 \mathrm{H}), 1.12-0.97(\mathrm{~m}, 4 \mathrm{H}), 0.94-0.76(\mathrm{~m}, 1 \mathrm{H}), 0.72(\mathrm{t}, J=7.0 \mathrm{~Hz}$, 3H). See spectrum.

${ }^{13} \mathbf{C}$ NMR $\left(100 \mathrm{MHz}, \mathrm{CDCl}_{3}\right) \delta_{\mathrm{C}}$ (major regioisomer reported) $196.9,179.6,179.3,136.3,136.0,133.6$, $128.7,128.7,128.5,128.0,127.7,48.7,43.3,42.5,40.9,39.9,31.9,29.0,27.2,22.4,13.9$. See spectrum. IR $v_{\max }: 2926,2854,1755,1693,1495,1446,1390,1341,1164,1066,1027 \mathrm{~cm}^{-1}$.

HRMS (ESI) m/z: [(M+Na) $\left.{ }^{+}\right]$calcd for $\mathrm{C}_{25} \mathrm{H}_{27} \mathrm{NO}_{3} 412.1883$, found 412.1890 . 


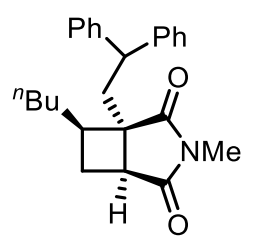

$>20: 1$ r.r.

General procedure 5. Using chlorocyclobutane 5 (150 mg, $0.65 \mathrm{mmol}, 12: 1$ r.r.), 1,1-diphenylethylene (345 $\mu \mathrm{L}, 1.95 \mathrm{mmol})$, DIPEA (140 $\mu \mathrm{L}, 0.78 \mathrm{mmol})$, acetic acid (30 $\mu \mathrm{L}, 0.52 \mathrm{mmol}), 4 \mathrm{CzIPN}$ (26 mg, $5 \mathrm{~mol} \%$ ) and $\mathrm{MeCN} /$ water $(4: 1,2.0 \mathrm{~mL}$ ), with a reaction time of 6 hours. Purification by column chromatography (98/2 petroleum ether/ethyl acetate) gave the title compound (149 $\mathrm{mg}, 61 \%)$ as a colourless solid.

${ }^{1} \mathbf{H}$ NMR $\left(400 \mathrm{MHz}, \mathrm{CDCl}_{3}\right) \delta_{\mathrm{H}} 7.22-7.00(\mathrm{~m}, 10 \mathrm{H}), 3.88(\mathrm{dd}, J=9.0,6.5 \mathrm{~Hz}, 1 \mathrm{H}), 2.84-2.71(\mathrm{~m}$, 1H), $2.78(\mathrm{~s}, 3 \mathrm{H}), 2.54-2.35(\mathrm{~m}, 2 \mathrm{H}), 2.35-2.19(\mathrm{~m}, 2 \mathrm{H}), 1.53-1.41(\mathrm{~m}, 1 \mathrm{H}), 1.36$ (ddd, $J=12.5$, 7.5, $5.0 \mathrm{~Hz}, 1 \mathrm{H}), 1.22-1.02(\mathrm{~m}, 4 \mathrm{H}), 0.96$ (ddd, $J=13.5,9.5,5.5 \mathrm{~Hz}, 1 \mathrm{H}), 0.77$ (t, $J=7.0 \mathrm{~Hz}, 3 \mathrm{H})$.

\section{See spectrum.}

${ }^{13} \mathrm{C}$ NMR $\left(100 \mathrm{MHz}, \mathrm{CDCl}_{3}\right) \delta_{\mathrm{C}} 179.7,179.3,143.6,143.6,128.8,128.5,127.6,127.6,127.0,126.6$, 52.2, 47.9, 42.3, 40.1, 39.0, 32.4, 29.1, 27.2, 24.6, 22.5, 14.0. See spectrum.

IR $v_{\max }: 3025,3060,2956,2928,2871,1769,1699,1450,1428,1378,1272,1056 \mathrm{~cm}^{-1}$.

HRMS (ESI) m/z: $\left[(\mathrm{M}+\mathrm{H})^{+}\right]$calcd for $\mathrm{C}_{31} \mathrm{H}_{33} \mathrm{NO}_{2} 452.2584$, found 452.2587 .

$\mathbf{M p}\left(\mathrm{CHCl}_{3}\right): 96-100{ }^{\circ} \mathrm{C}$.

$\left(1 R^{*}, 5 S^{*}, 7 R^{*}\right)-7-$ Butyl-1-(2,2-diphenylethyl)-3-azabicyclo[3.2.0]heptane-2,4-dione (34)

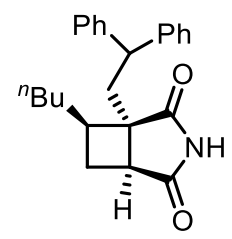

$>20: 1$ r.r.

General procedure 5. Using chlorocyclobutane 6 (140 mg, 0.65 mmol, 11:1 r.r.), 1,1-diphenylethylene (345 $\mu \mathrm{L}, 1.95 \mathrm{mmol})$, DIPEA ( $140 \mu \mathrm{L}, 0.78 \mathrm{mmol})$, acetic acid (30 $\mu \mathrm{L}, 0.52 \mathrm{mmol})$, 4CzIPN (26 mg, $5 \mathrm{~mol} \%)$ and $\mathrm{MeCN} /$ water $(4: 1,2.0 \mathrm{~mL})$, with a reaction time of 6 hours. Purification by column chromatography (90/10 toluene/petroleum ether to remove the catalyst, followed by 90/10 petroleum ether/ethyl acetate) gave the title compound (131 mg, 56\%) as a clear colourless oil. 
${ }^{1} \mathbf{H}$ NMR $\left(400 \mathrm{MHz}, \mathrm{CDCl}_{3}\right) \delta_{\mathrm{H}} 8.34(\mathrm{~s}, 1 \mathrm{H}), 7.29-6.99(\mathrm{~m}, 10 \mathrm{H}), 3.95(\mathrm{dd}, J=10.0,5.5 \mathrm{~Hz}, 1 \mathrm{H})$, $2.72(\mathrm{dd}, J=14.0,5.5 \mathrm{~Hz}, 1 \mathrm{H}), 2.51-2.35(\mathrm{~m}, 2 \mathrm{H}), 2.28(\mathrm{tt}, J=9.5,7.0 \mathrm{~Hz}, 1 \mathrm{H}), 2.15(\mathrm{dd}, J=10.5$, $5.5 \mathrm{~Hz}, 1 \mathrm{H}), 1.59-1.42(\mathrm{~m}, 2 \mathrm{H}), 1.22-1.04(\mathrm{~m}, 5 \mathrm{H}), 0.77(\mathrm{t}, J=7.0 \mathrm{~Hz}, 3 \mathrm{H})$. See spectrum.

${ }^{13} \mathrm{C}$ NMR $\left(100 \mathrm{MHz}, \mathrm{CDCl}_{3}\right) \delta_{\mathrm{C}} 179.9,179.9,143.9,143.5,129.0,128.5,127.9,127.6,127.1,126.6$, 53.8, 47.9, 42.2, 40.2, 40.2, 32.4, 29.1, 27.4, 22.5, 14.0. See spectrum.

IR $v_{\max }: 3214,3051,2955,2928,2855,1772,1708,1493,1341,1171,1032 \mathrm{~cm}^{-1}$.

HRMS (ESI) m/z: $\left[(\mathrm{M}+\mathrm{H})^{+}\right]$calcd for $\mathrm{C}_{31} \mathrm{H}_{33} \mathrm{NO}_{2} 452.2584$, found 452.2587 .

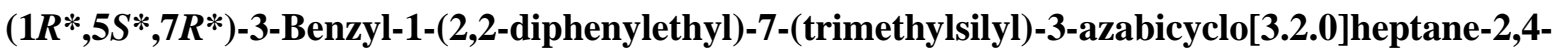
dione (35)

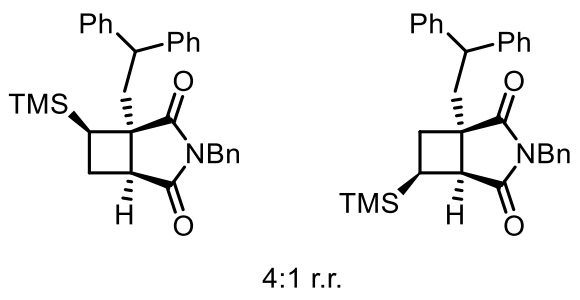

General procedure 5. Using chlorocyclobutane 7 (209 mg, $0.65 \mathrm{mmol}$, 4:1 r.r.), 1,1-diphenylethylene (345 $\mu \mathrm{L}, 1.95 \mathrm{mmol})$, DIPEA (140 $\mu \mathrm{L}, 0.78 \mathrm{mmol})$, acetic acid (30 $\mu \mathrm{L}, 0.52 \mathrm{mmol}), 4 \mathrm{CzIPN}$ (26 mg, $5 \mathrm{~mol} \%)$ and $\mathrm{MeCN} /$ water $(4: 1,2.0 \mathrm{~mL})$, with a reaction time of 6 hours. Purification by column chromatography (199/1 petroleum ether/ethyl acetate) gave the title compound ( $83 \mathrm{mg}, 27 \%)$ as a white solid.

${ }^{1} \mathbf{H} \mathbf{N M R}\left(400 \mathrm{MHz}, \mathrm{CDCl}_{3}\right) \delta_{\mathrm{H}}$ (major regioisomer reported) $7.71-7.61(\mathrm{~m}, 2 \mathrm{H}), 7.56-7.41(\mathrm{~m}, 3 \mathrm{H})$, $7.38-7.29(\mathrm{~m}, 3 \mathrm{H}), 7.29-7.08(\mathrm{~m}, 5 \mathrm{H}), 6.77-6.71(\mathrm{~m}, 2 \mathrm{H}), 4.81-4.58(\mathrm{~m}, 2 \mathrm{H}), 3.70(\mathrm{dd}, J=11.0$, $4.0 \mathrm{~Hz}, 1 \mathrm{H}), 2.74(\mathrm{dd}, J=14.0,4.0 \mathrm{~Hz}, 1 \mathrm{H}), 2.61(\mathrm{dd}, J=14.0,11.5 \mathrm{~Hz}, 1 \mathrm{H}), 2.52-2.44(\mathrm{~m}, 1 \mathrm{H}), 2.31$ $-2.25(\mathrm{~m}, 1 \mathrm{H}), 2.00(\mathrm{dd}, J=11.5,9.5 \mathrm{~Hz}, 1 \mathrm{H}), 1.78-1.70(\mathrm{~m}, 1 \mathrm{H}), 0.00(\mathrm{~s}, 9 \mathrm{H})$. See spectrum.

${ }^{13} \mathbf{C ~ N M R}\left(100 \mathrm{MHz}, \mathrm{CDCl}_{3}\right) \delta_{\mathrm{C}}$ (major regioisomer reported) 180.6, 178.8, 144.3, 143.0, 136.0, 130.0, 128.9, 128.6, 128.4, 127.7, 127.2, 126.9, 126.4, 50.2, 47.8, 42.6, 42.0, 41.7, 32.0, 21.8, -3.2. See spectrum.

IR $v_{\max }: 3474,2969,2950,1710,1430,1384,1341,1249,836,737,697 \mathrm{~cm}^{-1}$.

HRMS (ESI) m/z: $\left[(\mathrm{M}+\mathrm{Na})^{+}\right]$calcd for $\mathrm{C}_{30} \mathrm{H}_{33} \mathrm{NO}_{2} \mathrm{Si} 490.2173$, found 490.2190 . 


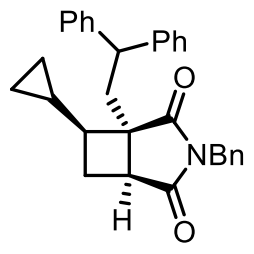

>20:1 r.r.

General procedure 5. Using chlorocyclobutane 8 (188 mg, $0.65 \mathrm{mmol}$, 9:1 r.r.), 1,1-diphenylethylene (345 $\mu \mathrm{L}, 1.95 \mathrm{mmol})$, DIPEA (140 $\mu \mathrm{L}, 0.78 \mathrm{mmol})$, acetic acid (30 $\mu \mathrm{L}, 0.52 \mathrm{mmol}), 4 \mathrm{CzIPN}(26 \mathrm{mg}$, $5 \mathrm{~mol} \%$ ) and $\mathrm{MeCN} /$ water $(4: 1,2.0 \mathrm{~mL}$ ), with a reaction time of 6 hours. Purification by column chromatography (98/2 petroleum ether/ethyl acetate) gave the title compound ( $223 \mathrm{mg}, 79 \%)$ as a white solid.

${ }^{1} \mathbf{H}$ NMR $\left(400 \mathrm{MHz}, \mathrm{CDCl}_{3}\right) \delta_{\mathrm{H}} 7.74-7.69(\mathrm{~m}, 2 \mathrm{H}), 7.59-7.51(\mathrm{~m}, 3 \mathrm{H}), 7.43(\mathrm{~d}, J=7.1 \mathrm{~Hz}, 2 \mathrm{H}), 7.39$ $-7.33(\mathrm{~m}, 3 \mathrm{H}), 7.32(\mathrm{dd}, J=5.0,2.0 \mathrm{~Hz}, 3 \mathrm{H}), 7.03(\mathrm{dd}, J=7.5,2.0 \mathrm{~Hz}, 2 \mathrm{H}), 4.88-4.67(\mathrm{~m}, 2 \mathrm{H}), 3.98$ (dd, $J=10.5,5.0 \mathrm{~Hz}, 1 \mathrm{H}), 3.02(\mathrm{dd}, J=14.0,5.0 \mathrm{~Hz}, 1 \mathrm{H}), 2.70-2.49(\mathrm{~m}, 2 \mathrm{H}), 2.32-2.16(\mathrm{~m}, 2 \mathrm{H})$, $1.58(\mathrm{ddd}, J=12.5,7.5,5.0 \mathrm{~Hz}, 1 \mathrm{H}), 0.79-0.69(\mathrm{~m}, 1 \mathrm{H}), 0.65-0.58(\mathrm{~m}, 1 \mathrm{H}), 0.44-0.35(\mathrm{~m}, 1 \mathrm{H})$, $0.23-0.13(\mathrm{~m}, 1 \mathrm{H}),-0.01(\mathrm{dd}, J=9.5,5.0 \mathrm{~Hz}, 1 \mathrm{H})$. See spectrum.

${ }^{13} \mathrm{C}$ NMR $\left(100 \mathrm{MHz}, \mathrm{CDCl}_{3}\right) \delta_{\mathrm{C}} 178.9,178.8,144.1,143.2,136.1,129.6,128.9,128.6,128.4,128.1$, 127.7, 127.4, 126.9, 126.4, 52.7, 47.8, 46.5, 42.4, 40.1, 38.7, 24.5, 11.5, 2.6, 2.1. See spectrum.

IR $v_{\max }: 3449,3083,3023,2931,1738,1690,1492,1394,1350,1206,741,695 \mathrm{~cm}^{-1}$.

HRMS (ESI) m/z: [(M+Na) $\left.{ }^{+}\right]$calcd for $\mathrm{C}_{30} \mathrm{H}_{29} \mathrm{NO}_{2} 458.2090$, found 458.2112.

$\mathbf{M p}\left(\mathrm{CHCl}_{3}\right): 122-125^{\circ} \mathrm{C}$.

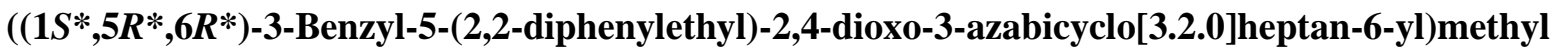
acetate (37)

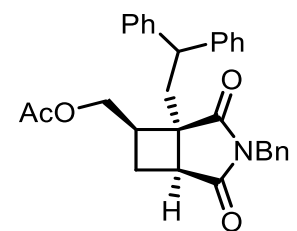

A

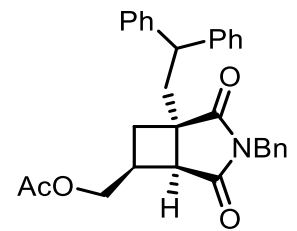

B

General procedure 5. Using chlorocyclobutane 9 (209 mg, $0.65 \mathrm{mmol}, 4: 1$ r.r.), 1,1-diphenylethylene (345 $\mu \mathrm{L}, 1.95 \mathrm{mmol})$, DIPEA (140 $\mu \mathrm{L}, 0.78 \mathrm{mmol})$, acetic acid (30 $\mu \mathrm{L}, 0.52 \mathrm{mmol}), 4 \mathrm{CzIPN}(26 \mathrm{mg}$, 
$5 \mathrm{~mol} \%$ ) and $\mathrm{MeCN} /$ water (4:1, $2.0 \mathrm{~mL}$ ), with a reaction time of 6 hours. Purification by column chromatography (90/10 toluene/petroleum ether to remove the catalyst, followed by $90 / 10$ to $70 / 30$ petroleum ether/ethyl acetate) gave the title compound (200 mg, 67\%) as a colourless solid.

${ }^{1} \mathbf{H}$ NMR $\left(400 \mathrm{MHz}, \mathrm{CDCl}_{3}\right) \delta_{\mathrm{H}}$ (mixture of regioisomers, $A+B$, reported) $7.48-7.40(\mathrm{~m}, 2 \mathrm{H}, \mathrm{A}+\mathrm{B})$, $7.27-7.03(\mathrm{~m}, 11 \mathrm{H}, \mathrm{A}+\mathrm{B}), 6.73-6.61(\mathrm{~m}, 2 \mathrm{H}, \mathrm{A}+\mathrm{B}), 4.62-4.45(\mathrm{~m}, 2 \mathrm{H}, \mathrm{A}+\mathrm{B}), 4.31(\mathrm{dd}, J=7.5,4.5$ $\mathrm{Hz}, 0.25 \mathrm{H}, \mathrm{B}$ ), 4.05 (dd, $J=12.0,5.0 \mathrm{~Hz}, 0.25 \mathrm{H}, \mathrm{B}), 3.95$ (dd, $J=12.0,6.5 \mathrm{~Hz}, 0.75 \mathrm{H}, \mathrm{A}), 3.81$ (dd, $J$ $=12.0,5.5 \mathrm{~Hz}, 1 \mathrm{H}, \mathrm{A}), 3.80-3.70(\mathrm{~m}, 0.25 \mathrm{H}, \mathrm{B}), 3.62(\mathrm{dd}, J=10.5,5.0 \mathrm{~Hz}, 0.75 \mathrm{H}, \mathrm{A}), 2.92-2.84$ (m, 0.25H, B), $2.74(\mathrm{dd}, J=14.0,5.0 \mathrm{~Hz}, 0.75 \mathrm{H}, \mathrm{A}), 2.68-2.55(\mathrm{~m}, 1 \mathrm{H}, \mathrm{A}+\mathrm{B}), 2.46-2.32(\mathrm{~m}, 2 \mathrm{H}$, A+B), $2.19-2.07$ (m, 0.25H, B), 2.04 (dd, $J=10.0,5.5 \mathrm{~Hz}, 0.75 \mathrm{H}, \mathrm{A}), 1.87$ (s, 2.25H, A), 1.84 (s, $0.75 \mathrm{H}, \mathrm{B}), 1.57$ (ddd, $J=13.0,8.0,5.5 \mathrm{~Hz}, 0.75 \mathrm{H}, \mathrm{A})$. See spectrum.

${ }^{13} \mathbf{C ~ N M R}\left(100 \mathrm{MHz}, \mathrm{CDCl}_{3}\right) \delta_{\mathrm{C}}$ (mixture of regioisomers reported) $178.3,178.2,171.3,170.6,170.5$, 170.2 , 143.9, 142.9, 136.0, 135.9, 129.6, 129.5, 128.9, 128.8, 128.8, 128.7, 128.7, 128.6, 128.5, 128.5, $128.3,128.3,127.9,127.8,127.7,127.5,127.4,127.3,127.0,126.5,65.0,63.4,57.6,50.9,47.5,47.8$, 44.3, 42.7, 42.6, 41.3, 40.4, 40.4, 39.0, 38.4, 23.7, 23.7, 20.7, 20.6. See spectrum.

IR $v_{\max }: 3029,2942,2251,1735,1695,1452,1391,1235,1167,1032,907,727,698 \mathrm{~cm}^{-1}$.

HRMS (ESI) m/z: [(M+Na) $\left.)^{+}\right]$calcd for $\mathrm{C}_{30} \mathrm{H}_{29} \mathrm{NO}_{4} 490.1989$, found 490.1994 .

$\mathbf{M p}\left(\mathrm{CHCl}_{3}\right): 107-110{ }^{\circ} \mathrm{C}$.

$\left(1 R^{*}, 5 S^{*}, 7 S^{*}\right)$-3-Benzyl-1-(2,2-diphenylethyl)-7-phenyl-3-azabicyclo[3.2.0]heptane-2,4-dione (38)

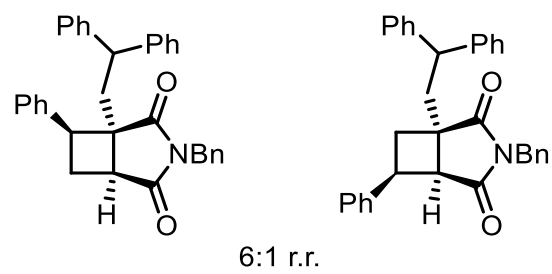

General procedure 5. Using chlorocyclobutane 10 (212 mg, 0.65 mmol, 7:1 r.r.), 1,1-diphenylethylene (345 $\mu \mathrm{L}, 1.95 \mathrm{mmol})$, DIPEA ( $140 \mu \mathrm{L}, 0.78 \mathrm{mmol})$, acetic acid (30 $\mu \mathrm{L}, 0.52 \mathrm{mmol}), 4 \mathrm{CzIPN}(26 \mathrm{mg}$, $5 \mathrm{~mol} \%)$ and $\mathrm{MeCN} /$ water $(4: 1,2.0 \mathrm{~mL})$, with a reaction time of 6 hours. Purification by column chromatography (98/2 petroleum ether/ethyl acetate) gave the title compound (185 $\mathrm{mg}, 60 \%)$ as a white solid.

${ }^{1} \mathbf{H}$ NMR $\left(500 \mathrm{MHz}, \mathrm{CDCl}_{3}\right) \delta_{\mathrm{H}}$ (major regioisomer reported) $7.44-7.32(\mathrm{~m}, 5 \mathrm{H}), 7.25-7.13(\mathrm{~m}$, $11 \mathrm{H}), 6.93-6.83(\mathrm{~m}, 4 \mathrm{H}), 4.50-4.39(\mathrm{~m}, 2 \mathrm{H}), 3.78(\mathrm{dd}, J=10.5,5.0 \mathrm{~Hz}, 1 \mathrm{H}), 3.69(\mathrm{dd}, J=10.0,8.0$ 
$\mathrm{Hz}, 1 \mathrm{H}), 2.95(\mathrm{dd}, J=14.0,5.0 \mathrm{~Hz}, 1 \mathrm{H}), 2.73-2.64(\mathrm{~m}, 2 \mathrm{H}), 2.27(\mathrm{dd}, J=10.0,5.0 \mathrm{~Hz}, 1 \mathrm{H}), 2.17$ (ddd, $J=13.5,8.0,5.0 \mathrm{~Hz}, 1 \mathrm{H}$ ). See spectrum.

${ }^{13} \mathbf{C ~ N M R}\left(100 \mathrm{MHz}, \mathrm{CDCl}_{3}\right) \delta_{\mathrm{C}}$ (major regioisomer reported) 178.5, 177.4, 143.9, 143.1, 137.6, 136.0, 129.7, 129.0, 128.6, 128.5, 128.4, 128.1, 127.8, 127.4, 127.3, 127.2, 127.1, 126.5, 54.9, 47.9, 47.6, 42.3, 40.7, 38.8, 25.4. See spectrum.

IR $v_{\max }: 3058,3030,2924,1765,1694,1493,1453,1391,1344,1167,1065,1032,738,696 \mathrm{~cm}^{-1}$.

HRMS (ESI) m/z: [(M+Na) $\left.{ }^{+}\right]$calcd for $\mathrm{C}_{33} \mathrm{H}_{29} \mathrm{NO}_{2}$ 494.2090, found 494.2088.

$\operatorname{Mp}\left(\mathrm{CHCl}_{3}\right): 142-145^{\circ} \mathrm{C}$.

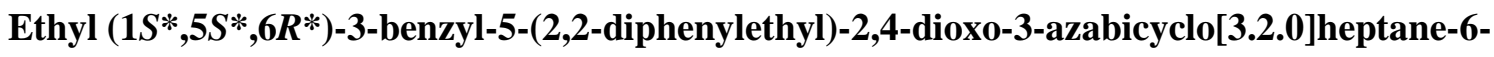
carboxylate (39)

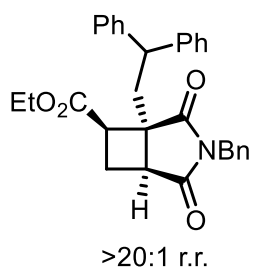

General procedure 5. Using chlorocyclobutane 11 (209 mg, 0.65 mmol, 4:1 r.r.), 1,1-diphenylethylene (345 $\mu \mathrm{L}, 1.95 \mathrm{mmol})$, DIPEA (140 $\mu \mathrm{L}, 0.78 \mathrm{mmol})$, acetic acid (30 $\mu \mathrm{L}, 0.52 \mathrm{mmol}), 4 \mathrm{CzIPN}(26 \mathrm{mg}$, $5 \mathrm{~mol} \%)$ and $\mathrm{MeCN} /$ water $(4: 1,2.0 \mathrm{~mL}$ ), with a reaction time of 6 hours. Purification by column chromatography (90/10 toluene/petroleum ether to remove the catalyst, followed by $90 / 10$ to $80 / 20$ petroleum ether/ethyl acetate) gave the title compound ( $200 \mathrm{mg}, 66 \%)$ as a clear colourless oil.

${ }^{1} \mathbf{H}$ NMR $\left(400 \mathrm{MHz}, \mathrm{CDCl}_{3}\right) \delta_{\mathrm{H}} 7.48-6.95(\mathrm{~m}, 13 \mathrm{H}), 6.68-6.59(\mathrm{~m}, 2 \mathrm{H}), 4.62-4.45(\mathrm{~m}, 2 \mathrm{H}), 4.09$ $-3.91(\mathrm{~m}, 2 \mathrm{H}), 3.56(\mathrm{dd}, J=11.0,4.5 \mathrm{~Hz}, 1 \mathrm{H}), 3.11$ (dd, $J=9.5,8.0 \mathrm{~Hz}, 1 \mathrm{H}), 2.83$ (ddd, $J=14.0,4.5$, $3.0 \mathrm{~Hz}, 1 \mathrm{H}), 2.51(\mathrm{dd}, J=14.0,11.0 \mathrm{~Hz}, 1 \mathrm{H}), 2.39-2.27(\mathrm{~m}, 1 \mathrm{H}), 2.19-2.08(\mathrm{~m}, 1 \mathrm{H}), 2.08-1.98(\mathrm{~m}$, $1 \mathrm{H}), 1.15(\mathrm{t}, J=7.0 \mathrm{~Hz}, 3 \mathrm{H})$. See spectrum.

${ }^{13} \mathrm{C}$ NMR $\left(100 \mathrm{MHz}, \mathrm{CDCl}_{3}\right) \delta_{\mathrm{C}} 177.7,177.4,170.8,143.8,142.7,135.9,129.5,129.0,128.7,128.5$, 128.2, 127.6, 127.2, 127.1, 126.6, 61.3, 52.1, 47.6, 44.8, 42.8, 40.2, 39.1, 23.0, 14.2. See spectrum. IR $v_{\max }: 3376,3028,2931,1698,1494,1452,1390,1342,1199,1160,1031,744,697 \mathrm{~cm}^{-1}$. HRMS (ESI) m/z: [(M+Na) $\left.{ }^{+}\right]$calcd for $\mathrm{C}_{30} \mathrm{H}_{29} \mathrm{NO}_{4} 490.1989$, found 490.2002 . 
$\left(3 \mathrm{a} R^{*}, 3 \mathrm{~b} S^{*}, 6 \mathrm{a} R^{*}, 6 \mathrm{~b} S^{*}\right)-2-B e n z y l-3 \mathrm{a}-(2,2-d i p h e n y l e t h y l) h e x a h y d r o c y c l o p e n t a[3,4]$ cyclobuta[1,2c]pyrrole-1,3(2H,3aH)-dione (40)
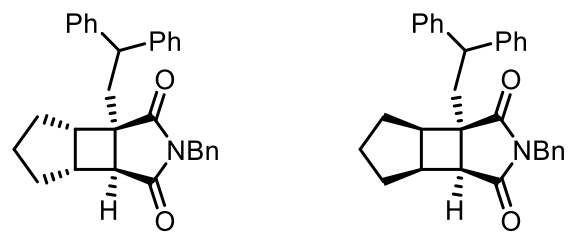

7:1 d.r.

General procedure 5. Using chlorocyclobutane 12 (188 mg, $0.65 \mathrm{mmol}, 12: 1$ d.r.), 1,1-diphenylethylene (345 $\mu \mathrm{L}, 1.95 \mathrm{mmol})$, DIPEA (140 $\mu \mathrm{L}, 0.78 \mathrm{mmol})$, acetic acid (30 $\mu \mathrm{L}, 0.52 \mathrm{mmol}), 4 \mathrm{CzIPN}$ (26 mg, $5 \mathrm{~mol} \%)$ and $\mathrm{MeCN} /$ water $(4: 1,2.0 \mathrm{~mL})$, with a reaction time of 6 hours. Purification by column chromatography ( $98 / 2$ petroleum ether/ethyl acetate) gave the title compound ( $224 \mathrm{mg}, 79 \%$ ) as a colourless solid.

${ }^{1} \mathbf{H}$ NMR $\left(400 \mathrm{MHz}, \mathrm{CDCl}_{3}\right) \delta_{\mathrm{H}}$ (major diastereomer reported) $7.55-7.48(\mathrm{~m}, 2 \mathrm{H}), 7.44-7.08(\mathrm{~m}$, $11 \mathrm{H}), 6.81-6.73(\mathrm{~m}, 2 \mathrm{H}), 4.66-4.50(\mathrm{~m}, 2 \mathrm{H}), 3.56(\mathrm{dd}, J=10.5,5.0 \mathrm{~Hz}, 1 \mathrm{H}), 2.76(\mathrm{t}, J=7.0 \mathrm{~Hz}$, $1 \mathrm{H}), 2.57-2.38(\mathrm{~m}, 3 \mathrm{H}), 2.01(\mathrm{dt}, J=11.5,5.5 \mathrm{~Hz}, 1 \mathrm{H}), 1.93(\mathrm{dd}, J=11.5,6.0 \mathrm{~Hz}, 1 \mathrm{H}), 1.67(\mathrm{~d}, J=$ $3.5 \mathrm{~Hz}, 1 \mathrm{H}), 1.61-1.45(\mathrm{~m}, 4 \mathrm{H})$. See spectrum.

${ }^{13} \mathrm{C}$ NMR $\left(100 \mathrm{MHz}, \mathrm{CDCl}_{3}\right) \delta_{\mathrm{C}}$ (major diastereomer reported) 182.5, 178.6, 144.1, 142.8, 136.3, 129.2, 128.9, 128.7, 128.4, 128.1, 127.6, 127.3, 127.0, 126.4, 47.9, 47.4, 46.8, 44.4, 42.4, 41.5, 33.0, $32.3,27.4,25.4$. See spectrum.

IR $v_{\max }: 3437,3058,3031,2942,2852,1763,1688,1493,1433,1392,1343,1053,1033,741,700 \mathrm{~cm}^{-1}$.

HRMS (ESI) m/z: [(M+Na) $\left.)^{+}\right]$calcd for $\mathrm{C}_{30} \mathrm{H}_{29} \mathrm{NO}_{2} 458.2090$, found 458.2108 .

$\mathbf{M p}\left(\mathrm{CHCl}_{3}\right): 137-140{ }^{\circ} \mathrm{C}$.

tert-Butyl $\left(1 S^{*}, 5 S^{*}\right)$-3-benzyl-5-(2,2-diphenylethyl)-2,4-dioxo-3-azaspiro[bicyclo[3.2.0]heptane6,4'-piperidine]-1'-carboxylate (41)

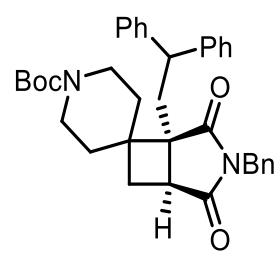

General procedure 5. Using chlorocyclobutane 13 (272 mg, $0.65 \mathrm{mmol}$, >20:1 r.r.), 1,1diphenylethylene ( $345 \mu \mathrm{L}, 1.95 \mathrm{mmol})$, DIPEA (140 $\mu \mathrm{L}, 0.78 \mathrm{mmol})$, acetic acid ( $30 \mu \mathrm{L}, 0.52 \mathrm{mmol})$, $4 \mathrm{CzIPN}(26 \mathrm{mg}, 5 \mathrm{~mol} \%)$ and MeCN/water (4:1, $2.0 \mathrm{~mL}$ ), with a reaction time of 6 hours. Purification 
by column chromatography (90/10 toluene/petroleum ether to remove the catalyst, followed by 90/10 to $80 / 20$ petroleum ether/ethyl acetate) gave the title compound (212 $\mathrm{mg}, 58 \%$ ) as a yellow oil.

${ }^{1} \mathbf{H}$ NMR $\left(500 \mathrm{MHz}, \mathrm{CDCl}_{3}\right) \delta_{\mathrm{H}} 7.57-7.53(\mathrm{~m}, 2 \mathrm{H}), 7.40-7.32(\mathrm{~m}, 3 \mathrm{H}), 7.25-7.13(\mathrm{~m}, 8 \mathrm{H}), 6.79-$ $6.73(\mathrm{~m}, 2 \mathrm{H}), 4.70-4.53$ (m, 2H), 4.00 (br. d, $J=14.0 \mathrm{~Hz}, 1 \mathrm{H}$ ), 3.80 (br. d, $J=13.5 \mathrm{~Hz}, 1 \mathrm{H}$ ), 3.61 (dd, $J=10.5,4.5 \mathrm{~Hz}, 1 \mathrm{H}), 2.74(\mathrm{t}, J=12.5 \mathrm{~Hz}, 1 \mathrm{H}), 2.68-2.48(\mathrm{~m}, 3 \mathrm{H}), 2.21-2.11(\mathrm{~m}, 2 \mathrm{H}), 1.79-1.62$ (m, 3H), $1.50-1.43(\mathrm{~m}, 2 \mathrm{H}), 1.46(\mathrm{~s}, 9 \mathrm{H})$. See spectrum.

${ }^{13}$ C NMR $\left(125 \mathrm{MHz}, \mathrm{CDCl}_{3}\right) \delta_{\mathrm{C}} 179.2,178.8,175.6,154.5,144.2,142.9,136.0,129.7,129.0,128.7$, $128.5,127.7,127.2,127.1,126.4,79.7,55.1,47.5,42.5,41.2,37.7,34.3,33.8,33.2,32.1,28.4$. See spectrum.

IR $v_{\max }: 2974,2938,2865,1712,1687,1455,1425,1387,1242,1171,1144,1032,732 \mathrm{~cm}^{-1}$.

HRMS (ESI) m/z: [(M+Na) $\left.{ }^{+}\right]$calcd for $\mathrm{C}_{22} \mathrm{H}_{27} \mathrm{~N}_{2} \mathrm{O}_{4} 441.1552$, found 441.1563 .

$\left(3 \mathrm{a} S^{*}, 4 \mathrm{a} R^{*}, 7 \mathrm{a} R^{*}\right)-4 \mathrm{a}-(2,2-D i p h e n y l e t h y l)-6-m e t h y l t e t r a h y d r o f u r o[2 ', 3 ': 1,4]$ cyclobuta[1,2c]pyrrole-5,7(2H,6H)-dione (42)

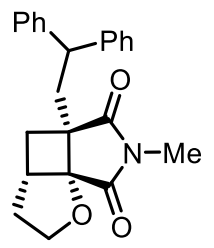

General procedure 5. Using chlorocyclobutane 14 (140 mg, $0.65 \mathrm{mmol}$, >20:1 d.r.), 1,1diphenylethylene ( $345 \mu \mathrm{L}, 1.95 \mathrm{mmol})$, DIPEA (140 $\mu \mathrm{L}, 0.78 \mathrm{mmol})$, acetic acid (30 $\mu \mathrm{L}, 0.52 \mathrm{mmol})$, 4CzIPN (26 mg, $5 \mathrm{~mol} \%$ ) and MeCN/water (4:1, $2.0 \mathrm{~mL}$ ), with a reaction time of 6 hours. Purification by column chromatography ( $90 / 10$ toluene/petroleum ether to remove the catalyst, followed by $90 / 10$ to $70 / 30$ petroleum ether/ethyl acetate) gave the title compound (221 $\mathrm{mg}, 94 \%)$ as a colourless powder.

${ }^{1} \mathbf{H}$ NMR $\left(400 \mathrm{MHz}, \mathrm{CDCl}_{3}\right) \delta_{\mathrm{H}} 7.28-7.09(\mathrm{~m}, 10 \mathrm{H}), 4.56-4.50(\mathrm{~m}, 1 \mathrm{H}), 4.40(\mathrm{dd}, J=11.0,4.5 \mathrm{~Hz}$, $1 \mathrm{H}), 4.22$ (ddd, $J=10.5,9.0,6.0 \mathrm{~Hz}, 1 \mathrm{H}), 2.99-2.91(\mathrm{~m}, 1 \mathrm{H}), 2.87(\mathrm{dd}, J=14.5,11.0 \mathrm{~Hz}, 1 \mathrm{H}), 2.54$ (s, 3H), $2.38-2.28(\mathrm{~m}, 2 \mathrm{H}), 2.08(\mathrm{ddt}, J=12.5,10.5,8.0 \mathrm{~Hz}, 1 \mathrm{H}), 1.81(\mathrm{ddt}, J=13.0,6.0,2.0 \mathrm{~Hz}, 1 \mathrm{H})$, $1.65(\mathrm{dd}, J=13.5,6.5 \mathrm{~Hz}, 1 \mathrm{H})$. See spectrum.

${ }^{13} \mathrm{C}$ NMR $\left(100 \mathrm{MHz}, \mathrm{CDCl}_{3}\right) \delta_{\mathrm{C}} 178.8,175.6,144.5,142.5,128.5,128.5,128.4,127.4,126.8,126.4$, 86.6, 72.2, 48.9, 46.9, 41.4, 37.5, 33.7, 31.3, 24.6. See spectrum.

IR $v_{\max }: 2941,1776,1706,1449,1031,702 \mathrm{~cm}^{-1}$.

HRMS (ESI) m/z: [(M+Na) $\left.{ }^{+}\right]$calcd for $\mathrm{C}_{23} \mathrm{H}_{23} \mathrm{NO}_{3} 384.1576$, found 384.1563 .

$\mathbf{M p}\left(\mathrm{CHCl}_{3}\right): 128-130^{\circ} \mathrm{C}$. 

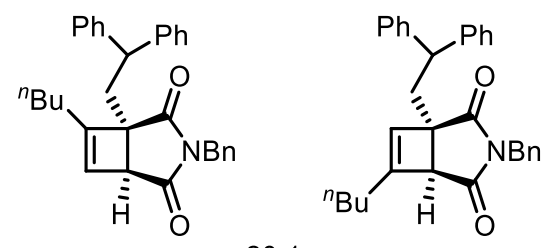

$>20: 1$ r.r.

General procedure 5. Using chlorocyclobutene 3 (197 mg, 0.65 mmol, 10:1 r.r.), 1,1-diphenylethylene (345 $\mu \mathrm{L}, 1.95 \mathrm{mmol})$, DIPEA (140 $\mu \mathrm{L}, 0.78 \mathrm{mmol})$, acetic acid $(30 \mu \mathrm{L}, 0.52 \mathrm{mmol}), 4 \mathrm{CzIPN}(26 \mathrm{mg}$, $5 \mathrm{~mol} \%)$ and $\mathrm{MeCN} /$ water $(4: 1,2.0 \mathrm{~mL})$, with a reaction time of 6 hours. Purification by column chromatography (98/2 petroleum ether/ethyl acetate) gave the title compound (224 $\mathrm{mg}, 77 \%)$ as a clear colourless oil.

${ }^{1} \mathbf{H}$ NMR $\left(400 \mathrm{MHz}, \mathrm{CDCl}_{3}\right) \delta_{\mathrm{H}}$ (major regioisomer reported) $7.45-7.08(\mathrm{~m}, 13 \mathrm{H}), 6.95(\mathrm{dd}, J=6.5$, $3.0 \mathrm{~Hz}, 2 \mathrm{H}), 5.90(\mathrm{~d}, J=1.5 \mathrm{~Hz}, 1 \mathrm{H}), 4.62-4.47(\mathrm{~m}, 2 \mathrm{H}), 3.91$ (dd, $J=10.0,5.5 \mathrm{~Hz}, 1 \mathrm{H}), 2.91$ (dd, $J$ $=14.0,5.5 \mathrm{~Hz}, 1 \mathrm{H}), 2.52(\mathrm{~d}, J=1.5 \mathrm{~Hz}, 1 \mathrm{H}), 2.47(\mathrm{dd}, J=14.0,10.0 \mathrm{~Hz}, 1 \mathrm{H}), 2.08-2.01(\mathrm{~m}, 2 \mathrm{H}), 1.47$ $-1.18(\mathrm{~m}, 4 \mathrm{H}), 0.87$ (t, $J=7.0 \mathrm{~Hz}, 3 \mathrm{H})$. See spectrum.

${ }^{13} \mathbf{C}$ NMR $\left(100 \mathrm{MHz}, \mathrm{CDCl}_{3}\right) \delta_{\mathrm{C}}$ (major regioisomer reported) $176.6,175.1,157.4,143.9,143.6,136.0$, $128.9,128.9,128.6,128.5,127.9,127.9,127.8,127.0,127.5,126.6,58.2,48.0,47.9,42.0,34.8,27.8$, 27.2, 22.3, 13.8. See spectrum.

IR $v_{\max }: 2928,1699,1452,1388,1166,701 \mathrm{~cm}^{-1}$.

HRMS (ESI) m/z: $\left[(\mathrm{M}+\mathrm{H})^{+}\right]$calcd for $\mathrm{C}_{31} \mathrm{H}_{31} \mathrm{NO}_{2} 450.2433$, found 450.2418 .

Ethyl 3-((1S*,5S*)-3-benzyl-7-butyl-2,4-dioxo-3-azabicyclo[3.2.0]hept-6-en-1-yl)propanoate (44)

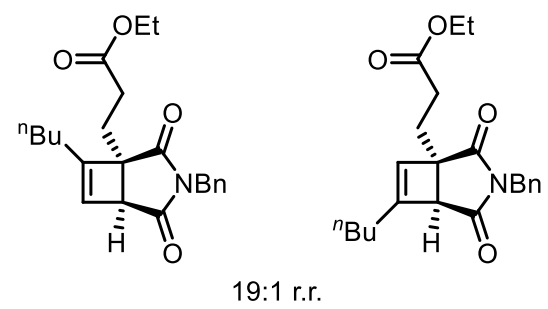

General procedure 5. Using chlorocyclobutene 3 (197 mg, $0.65 \mathrm{mmol}, 10: 1$ r.r.), ethyl acrylate ( $212 \mu \mathrm{L}$, $1.95 \mathrm{mmol})$, DIPEA ( $140 \mu \mathrm{L}, 0.78 \mathrm{mmol})$, acetic acid ( $30 \mu \mathrm{L}, 0.52 \mathrm{mmol}), 4 \mathrm{CzIPN}(26 \mathrm{mg}, 5 \mathrm{~mol} \%)$ and $\mathrm{MeCN} /$ water $(4: 1,2.0 \mathrm{~mL})$, with a reaction time of 6 hours. Purification by column chromatography (90/10 toluene/petroleum ether to remove the catalyst, followed by $95 / 5$ petroleum ether/ethyl acetate) gave the title compound (124 mg, 52\%) as a clear colourless oil. 
${ }^{1} \mathbf{H} \mathrm{NMR}\left(500 \mathrm{MHz}, \mathrm{CDCl}_{3}\right) \delta_{\mathrm{H}}$ (major regioisomer reported) $7.33-7.22(\mathrm{~m}, 5 \mathrm{H}), 6.06(\mathrm{~d}, J=1.0 \mathrm{~Hz}$, $1 \mathrm{H}), 4.68-4.56(\mathrm{~m}, 2 \mathrm{H}), 4.18-4.05(\mathrm{~m}, 2 \mathrm{H}), 3.35(\mathrm{~d}, J=1.0 \mathrm{~Hz}, 1 \mathrm{H}), 2.40-2.26(\mathrm{~m}, 3 \mathrm{H}), 2.25-$ $2.10(\mathrm{~m}, 1 \mathrm{H}), 2.12-2.02(\mathrm{~m}, 2 \mathrm{H}), 1.46-1.30(\mathrm{~m}, 2 \mathrm{H}), 1.30-1.11(\mathrm{~m}, 5 \mathrm{H}), 0.85(\mathrm{t}, J=7.5 \mathrm{~Hz}, 3 \mathrm{H})$. See spectrum.

${ }^{13} \mathbf{C} \mathbf{N M R}\left(125 \mathrm{MHz}, \mathrm{CDCl}_{3}\right) \delta_{\mathrm{C}}$ (major regioisomer reported) $176.4,175.0,172.5,156.9,135.9,128.6$, 128.2, 128.1, 127.7, 60.8, 57.8, 47.8, 42.0, 29.8, 27.7, 27.7, 23.9, 22.2, 14.1, 13.7. See spectrum. IR $v_{\max }: 2927,1765,1701,1428,1389,1341,701 \mathrm{~cm}^{-1}$.

HRMS (ESI) m/z: [(M+Na) $\left.{ }^{+}\right]$calcd for $\mathrm{C}_{22} \mathrm{H}_{27} \mathrm{NO}_{4} 392.1838$, found 392.1827. 


\subsection{Deuteration Studies}

When the reaction of $\mathbf{4}$ with ethyl acrylate was performed with $\mathrm{D}_{2} \mathrm{O}$ in place of water, no deuterium incorporation $\alpha$ to the ester group in Giese addition product $\mathbf{2 4}$ was observed (Scheme S3). This implies that the intermediate $\alpha$-carbonyl radical does not undergo single-electron reduction to an anion followed by protonation. Therefore, we propose that the $\alpha$-carbonyl radical undergoes a hydrogen atom transfer (HAT) with DIPEA (or the radical cation of DIPEA) to generate the Giese addition product.

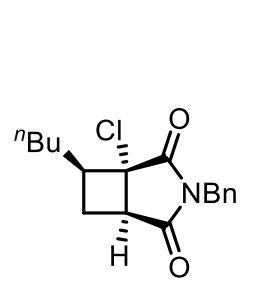

4

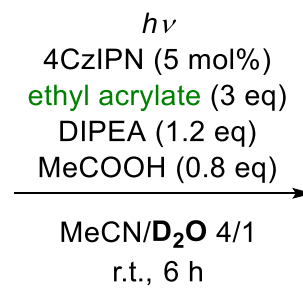

r.t., $6 \mathrm{~h}$

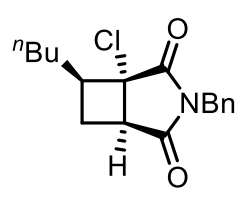

$4(13 \%)$

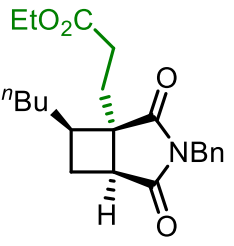

$24(54 \%)$

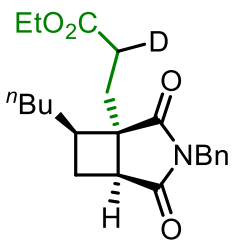

24-d (0\%)

Scheme S3. No deuteration $\alpha$ to the ester group signifies that the radical formed is not reduced to the anion, but instead undergoes HAT with DIPEA or DIPEA ${ }^{*+}$. 


\subsection{Cyclic Voltammetry}

The effect of water on the reduction potential of succinimide was investigated by cyclic voltammetry. First, background voltammograms of $\mathrm{MeCN}$ and wet $\mathrm{MeCN}$ were recorded (Figure S3), which showed that no reduction peaks occur in the region between $0 \mathrm{~V}$ and $-2.5 \mathrm{~V}$. Next, the reduction potential of 4CzIPN was recorded so that a direct comparison of reduction potentials could be made (Figure S4).

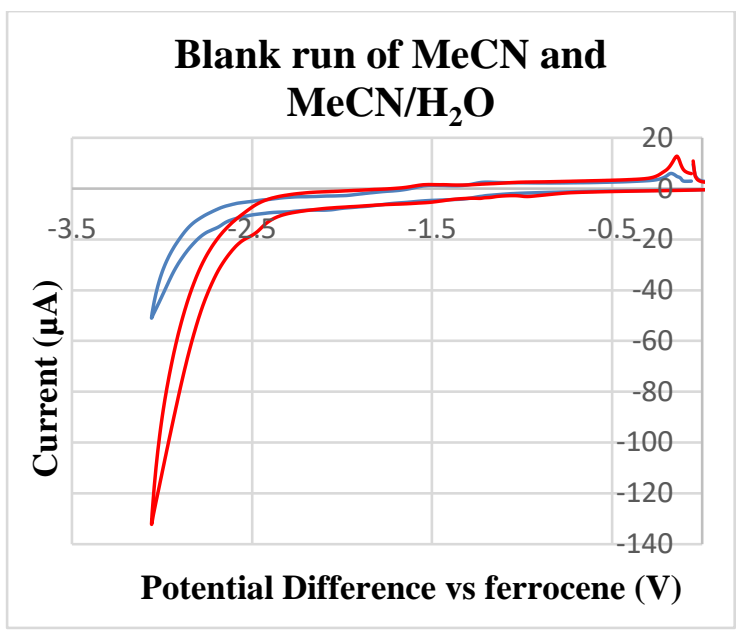

Figure S3. Blank run of dry $\mathrm{MeCN}$ (blue) and MeCN doped with 2 drops of water (red) with tetrabutylammonium hexafluorophosphate as the electrolyte $(0.1 \mathrm{M})$ at a scan rate of $0.1 \mathrm{~V} \mathrm{~s}^{-1}$, referenced to ferrocene using a glassy carbon electrode.

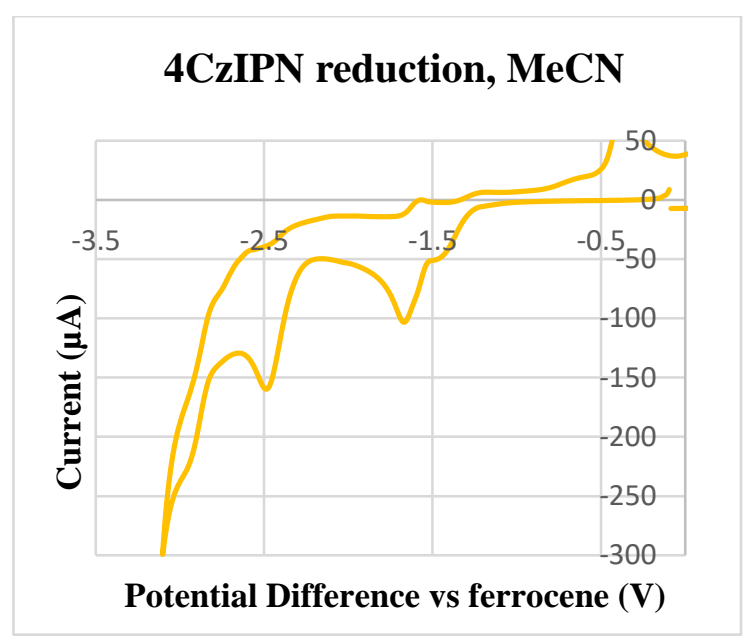

Figure S4. 4CzIPN (3 mM) run in dry $\mathrm{MeCN}$ (yellow) with tetra-butylammonium hexafluorophosphate as the electrolyte $(0.1 \mathrm{M})$ at a scan rate of $0.1 \mathrm{~V} \mathrm{~s}^{-1}$, referenced to ferrocene using a glassy carbon electrode. Reductive maxima = $-2.45 \mathrm{~V}$.

When the voltammograms of compound 4 (Figure S5) and hydrodechlorinated-4 (Figure S6) were compared in dry $\mathrm{MeCN}$ and wet $\mathrm{MeCN}$, a significant difference was observed in their reduction potentials. It was found that the presence of water resulted in a shift in the reduction peaks to more positive potentials for both $\mathbf{4}$ and hydrodechlorinated-4, therefore facilitating single-electron reduction.

The similar reduction potentials of $\mathbf{4}$ and hydrodechlorinated-4 suggest that the reductive dehalogenation proceeds via a two-step process involving initial reduction of the imide carbonyl followed by chloride elimination, rather than direct reduction of the $\mathrm{C}-\mathrm{Cl}$ bond. 


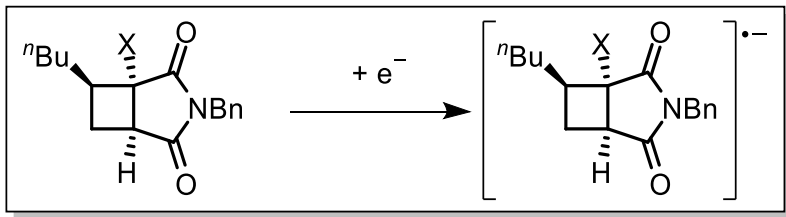

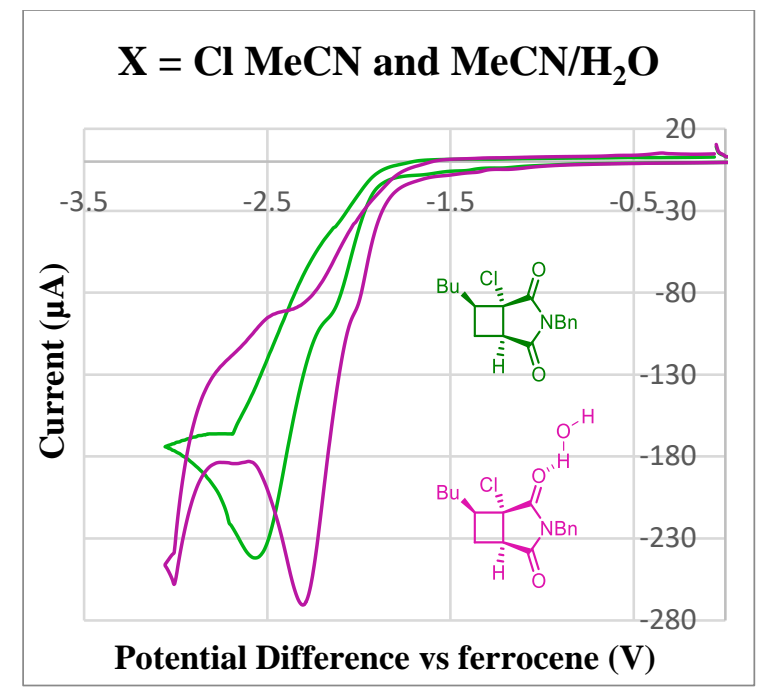

Figure S5. Compound 4 run in dry MeCN (green) and in MeCN doped with 2 drops of water (pink) (5 $\mathrm{mM}$ with tetra-butylammonium hexafluorophosphate as the electrolyte $(0.1 \mathrm{M})$ at a scan rate of $0.1 \mathrm{~V} \mathrm{~s}^{-1}$, referenced to ferrocene using a glassy carbon electrode. Respective reductive maxima taken as $-2.53 \mathrm{~V}$ (green) and $-2.29 \mathrm{~V}$ (pink).

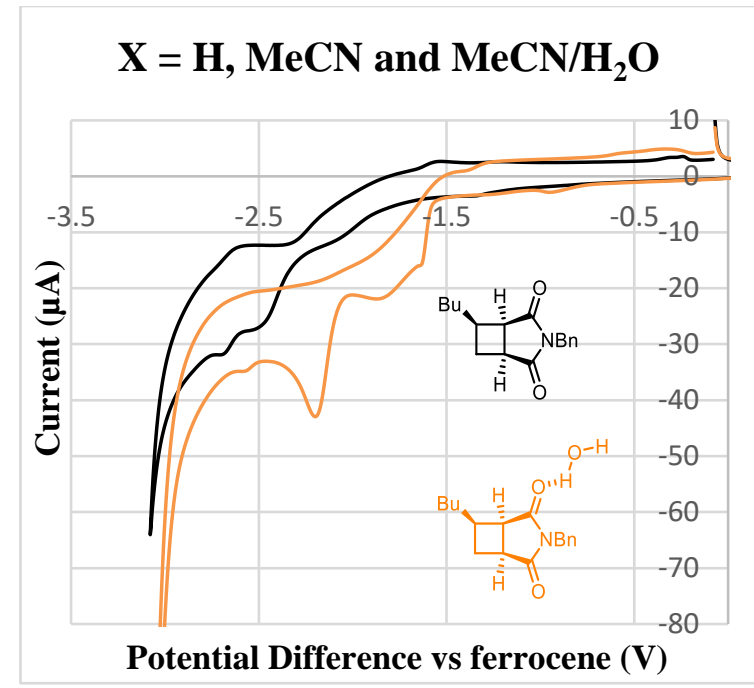

Figure S6. Hydrodechlorinated-4 run in dry $\mathrm{MeCN}$ (black) and in MeCN doped with 2 drops of water (orange) $(5 \mathrm{mM})$ with tetra-butylammonium hexafluorophosphate as the electrolyte $(0.1 \mathrm{M})$ at a scan rate of $0.1 \mathrm{~V} \mathrm{~s}^{-1}$, referenced to ferrocene using a glassy carbon electrode. Respective reductive maxima taken as $-2.52 \mathrm{~V}$ (black) and $-2.20 \mathrm{~V}$ (orange). 


\section{Succinimide Functionalisations}

$\left(1 S^{*}, 2 R^{*}, 3 R^{*}\right)-2-($ Benzylcarbamoyl)-3-butyl-2-(2,2-diphenylethyl)cyclobutane-1-carboxylic acid (52)

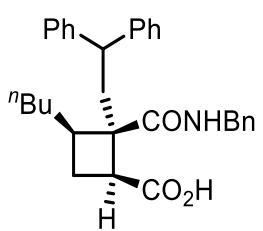

A solution of compound $15(50 \mathrm{mg}, 0.11 \mathrm{mmol})$ and $\mathrm{KOH}(31 \mathrm{mg}, 0.55 \mathrm{mmol})$ in ethanol (1.1 mL) was stirred at $60{ }^{\circ} \mathrm{C}$ for 4 hours. The solution was diluted with $1 \mathrm{M}$ aqueous $\mathrm{HCl}$ until the solution reached $\mathrm{pH}$ 7. The solvent was removed in vacuo and the residue was diluted in ethyl acetate $(10 \mathrm{~mL})$ and water $(10 \mathrm{~mL})$. The layers were separated and the aqueous layer was extracted with ethyl acetate $(2 \times 10 \mathrm{~mL})$. The combined organic extracts were dried over $\mathrm{MgSO}_{4}$ and concentrated in vacuo. Purification by column chromatography (80/20 to 70/30 petroleum ether/ethyl acetate) gave the title compound (42 $\mathrm{mg}, 81 \%)$ as a colourless solid.

${ }^{1} \mathbf{H}$ NMR $\left(400 \mathrm{MHz}, \mathrm{CDCl}_{3}\right) \delta_{\mathrm{H}} 7.31-7.02(\mathrm{~m}, 15 \mathrm{H}), 5.42(\mathrm{dd}, J=7.0,4.0 \mathrm{~Hz}, 1 \mathrm{H}), 4.45-4.33(\mathrm{~m}$, 2H), $3.54(\mathrm{dd}, J=14.5,4.0 \mathrm{~Hz}, 1 \mathrm{H}), 2.94(\mathrm{dd}, J=10.5,9.0 \mathrm{~Hz}, 1 \mathrm{H}), 2.70(\mathrm{dd}, J=15.0,8.0 \mathrm{~Hz}, 1 \mathrm{H})$, $2.56(\mathrm{dd}, J=15.0,4.5 \mathrm{~Hz}, 1 \mathrm{H}), 2.31-2.03(\mathrm{~m}, 3 \mathrm{H}), 1.32-1.23(\mathrm{~m}, 1 \mathrm{H}), 1.21-1.09(\mathrm{~m}, 1 \mathrm{H}), 0.99$ (ddd, $J=12.5,7.5,4.5 \mathrm{~Hz}, 1 \mathrm{H}), 0.80(\mathrm{t}, J=7.0 \mathrm{~Hz}, 3 \mathrm{H})$. See spectrum.

${ }^{13}$ C NMR $\left(100 \mathrm{MHz}, \mathrm{DMSO}-d_{6}\right) \delta_{\mathrm{C}} 187.2,172.2,145.5,145.3,137.6,129.0,128.8,128.6,128.4,127.9$, 127.6, 127.5, 126.5, 126.5, 58.9, 47.3, 46.8, 44.8, 44.2, 43.9, 31.3, 29.1, 27.8, 22.6, 14.0. See spectrum. IR $v_{\max }: 3375,2929,1717,1595,1544,1453,1440,1238,1223,1211,699 \mathrm{~cm}^{-1}$.

HRMS (ESI) m/z: [(M+Na) $\left.{ }^{+}\right]$calcd for $\mathrm{C}_{31} \mathrm{H}_{35} \mathrm{NO}_{3} 492.2509$, found 492.2511 .

$\mathrm{Mp}\left(\mathrm{CHCl}_{3}\right): 150-152{ }^{\circ} \mathrm{C}$. 


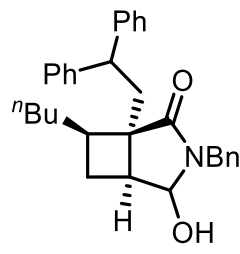

$10: 1$ d.r.

A solution of 15 (30 mg, $0.066 \mathrm{mmol})$ and $\mathrm{NaBH}_{4}(13 \mathrm{mg}, 0.33 \mathrm{mmol})$ in ethanol $(0.6 \mathrm{~mL})$ and water $(0.4 \mathrm{~mL})$ was stirred at $40{ }^{\circ} \mathrm{C}$ for 16 hours. The solution was diluted with ethyl acetate $(20 \mathrm{~mL})$ and washed with $1 \mathrm{M}$ aqueous $\mathrm{HCl}(3 \times 10 \mathrm{~mL})$. The organic layer was dried over $\mathrm{MgSO}_{4}$ and concentrated in vacuo. Purification by column chromatography (9/1 petroleum ether/ethyl acetate) gave the title compound (22 $\mathrm{mg}, 72 \%)$ as a colourless solid.

${ }^{1} \mathbf{H}$ NMR $\left(400 \mathrm{MHz}, \mathrm{CDCl}_{3}\right) \delta_{\mathrm{H}}$ (major diastereoisomer reported) $7.44-7.38(\mathrm{~m}, 2 \mathrm{H}), 7.37-7.23(\mathrm{~m}$, $3 \mathrm{H}), 7.20-6.93(\mathrm{~m}, 8 \mathrm{H}), 6.73-6.67(\mathrm{~m}, 2 \mathrm{H}), 4.68(\mathrm{~d}, J=14.0 \mathrm{~Hz}, 1 \mathrm{H}), 4.08(\mathrm{~d}, J=14.0 \mathrm{~Hz}, 1 \mathrm{H})$, $4.00-3.93(\mathrm{~m}, 1 \mathrm{H}), 3.56(\mathrm{dd}, J=11.5,4.0 \mathrm{~Hz}, 1 \mathrm{H}), 2.53(\mathrm{dd}, J=14.0,4.0 \mathrm{~Hz}, 1 \mathrm{H}), 2.38(\mathrm{dd}, J=14.0$, $11.5 \mathrm{~Hz}, 1 \mathrm{H}), 2.11-1.97(\mathrm{~m}, 3 \mathrm{H}), 1.85(\mathrm{dt}, J=12.0,9.0 \mathrm{~Hz}, 1 \mathrm{H}), 1.61-1.47(\mathrm{~m}, 1 \mathrm{H}), 1.38(\mathrm{ddd}, J=$ 12.0, 9.0, 7.0 Hz, 1H), $1.24-1.04(\mathrm{~m}, 5 \mathrm{H}), 0.79(\mathrm{t}, J=6.0 \mathrm{~Hz}, 3 \mathrm{H})$. See spectrum.

${ }^{13} \mathrm{C}$ NMR $\left(100 \mathrm{MHz}, \mathrm{CDCl}_{3}\right) \delta_{\mathrm{C}}$ (major diastereoisomer reported) $174.4,145.4,142.8,137.3,129.5$, $128.8,128.4,128.4,128.2$, 127.8, 127.2, 126.6, 126.1, 81.6, 53.9, 47.9, 43.5, 41.6, 40.9, 37.9, 31.9, 29.4, 22.7, 22.4, 14.1. See spectrum.

IR $v_{\max }: 3330,2924,2855,1646,1494,1451,1294,1100,1073,1032 \mathrm{~cm}^{-1}$.

HRMS HRMS (ESI) m/z: [(M+Na) $\left.)^{+}\right]$calcd for $\mathrm{C}_{31} \mathrm{H}_{35} \mathrm{NO}_{2} 476.2560$, found 476.2541.

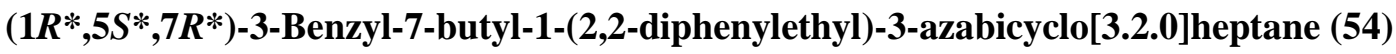

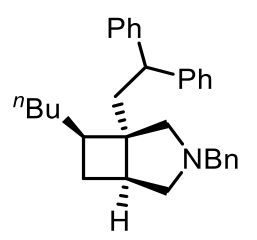

Compound 15 (53 mg, $0.11 \mathrm{mmol})$ was dissolved in THF $(0.5 \mathrm{~mL})$ in a flame dried Schlenk tube and added to a suspension of lithium aluminium hydride (1 M THF, $0.27 \mathrm{~mL})$ at $0{ }^{\circ} \mathrm{C}$ under a nitrogen atmosphere. The mixture was then stirred at reflux for 3 hours before being cooled to $0{ }^{\circ} \mathrm{C}$ and quenched with $30 \%$ aqueous $\mathrm{NaOH}(2 \mathrm{~mL})$. The precipitate was removed by filtration and the filtrate was 
concentrated in vacuo. Purification by column chromatography ( $95 / 5$ to $90 / 10$ petroleum ether/ethyl acetate) gave the title compound ( $32 \mathrm{mg}, 64 \%$ ) as a colourless oil.

${ }^{1} \mathbf{H}$ NMR $\left(400 \mathrm{MHz}, \mathrm{CDCl}_{3}\right) \delta_{\mathrm{H}} 7.30-7.18(\mathrm{~m}, 13 \mathrm{H}), 7.07(\mathrm{~d}, J=7.0 \mathrm{~Hz}, 2 \mathrm{H}), 3.78(\mathrm{t}, J=7.0 \mathrm{~Hz}, 1 \mathrm{H})$, $3.45(\mathrm{~s}, 2 \mathrm{H}), 2.65(\mathrm{~d}, J=10.0 \mathrm{~Hz}, 1 \mathrm{H}), 2.50-2.39(\mathrm{~m}, 2 \mathrm{H}), 2.20(\mathrm{dd}, J=14.0,7.0 \mathrm{~Hz}, 1 \mathrm{H}), 2.08-1.94$ $(\mathrm{m}, 2 \mathrm{H}), 1.88-1.81(\mathrm{~m}, 2 \mathrm{H}), 1.58(\mathrm{~d}, J=10.0 \mathrm{~Hz}, 1 \mathrm{H}), 1.42-1.31(\mathrm{~m}, 1 \mathrm{H}), 1.23-1.15(\mathrm{~m}, 2 \mathrm{H}), 1.13$ $-1.05(\mathrm{~m}, 2 \mathrm{H}), 1.02-0.93(\mathrm{~m}, 1 \mathrm{H}), 0.85-0.80(\mathrm{~m}, 1 \mathrm{H}), 0.76(\mathrm{t}, J=7.5 \mathrm{~Hz}, 3 \mathrm{H})$. See spectrum.

${ }^{13} \mathrm{C}$ NMR $\left(100 \mathrm{MHz}, \mathrm{CDCl}_{3}\right) \delta_{\mathrm{C}} 145.8,140.1,128.6,128.5,128.1,127.9,126.6,126.2,60.2,60.2,59.3$, 50.1, 48.8, 46.8, 40.9, 39.9, 29.9, 29.5, 28.5, 22.9, 14.3. See spectrum.

IR $v_{\max }: 3061,3026,2953,2921,2652,2783,1598,1493,1451,1216,1028,751,696 \mathrm{~cm}^{-1}$.

HRMS (ESI) m/z: [(M+Na) $\left.{ }^{+}\right]$calcd for $\mathrm{C}_{31} \mathrm{H}_{38} \mathrm{~N}$ 424.2999, found 424.3011 .

\section{Methyl $\left(1 S^{*}, 2 R^{*}, 3 R^{*}\right)-3$-butyl-2-(2,2-diphenylethyl)-2-((methoxycarbonyl)amino)cyclobutane-1- carboxylate (55)}
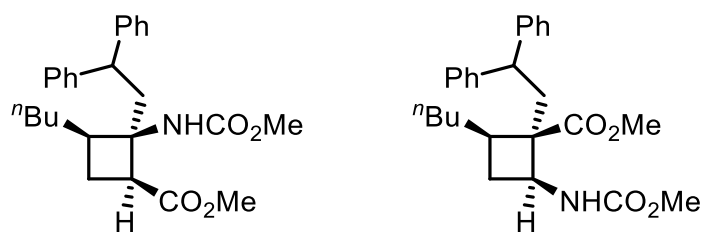

$7: 1$ r.r.

Compound 34 (15 mg, $0.041 \mathrm{mmol}$, >20:1 r.r.) was added to a solution of 1,3-dichloro-5,5dimethylhydantoin (DCDMH, $18 \mathrm{mg}, 0.082 \mathrm{mmol})$ in dry methanol $(0.2 \mathrm{~mL})$ in a microwave vial. To the resulting solution was added DBU $(19 \mu \mathrm{L}, 0.12 \mathrm{mmol})$ the mixture was stirred at $100{ }^{\circ} \mathrm{C}$ for 2 hours under microwave irradiation. The solvent was removed in vacuo and the crude material was diluted with EtOAc $(10 \mathrm{~mL})$ and washed with water $(2 \times 10 \mathrm{~mL})$ and brine $(10 \mathrm{~mL})$. The organic extracts were dried over $\mathrm{MgSO}_{4}$ and concentrated in vacuo. Purification by column chromatography (19/1 - 9/1 petroleum ether/ethyl acetate) gave the title compound (10 mg, 57\%) as a white solid.

${ }^{1} \mathbf{H}$ NMR $\left(400 \mathrm{MHz}, \mathrm{CDCl}_{3}\right) \delta_{\mathrm{H}}$ (major regioisomer reported) $7.21-7.15(\mathrm{~m}, 8 \mathrm{H}), 7.07$ (ddd, $J=8.0$, 4.5, $2.5 \mathrm{~Hz}, 2 \mathrm{H}$ ), 6.00 (br. s, 1H), 3.89 (t, $J=7.0 \mathrm{~Hz}, 1 \mathrm{H}$ ), 3.46 (s, 3H), 3.44 (s, 3H), 3.07 - 2.99 (m, $1 \mathrm{H}), 2.74-2.59(\mathrm{~m}, 2 \mathrm{H}), 2.09-1.99(\mathrm{~m}, 2 \mathrm{H}), 1.71(\mathrm{dd}, J=9.5,1.5 \mathrm{~Hz}, 1 \mathrm{H}), 1.25-1.03(\mathrm{~m}, 5 \mathrm{H}), 0.98$ $-0.86(\mathrm{~m}, 1 \mathrm{H}), 0.76(\mathrm{t}, J=7.0 \mathrm{~Hz}, 3 \mathrm{H})$. See spectrum.

${ }^{13} \mathbf{C}$ NMR $\left(100 \mathrm{MHz}, \mathrm{CDCl}_{3}\right) \delta_{\mathrm{C}}$ (major regioisomer reported) $173.5,155.9,144.9,144.5,128.5,128.4$, 128.1, 127.9, 126.1, 126.1, 62.3, 51.7, 51.6, 47.4, 44.5, 44.4, 43.8, 29.1, 28.7, 27.1, 22.6, 14.1. See spectrum. 
IR $v_{\max }: 3670,3354,2953,1728,1539,1504,1258,1230,1057 \mathrm{~cm}^{-1}$.

HRMS (ESI) m/z: [(M+Na) $\left.{ }^{+}\right]$calcd for $\mathrm{C}_{26} \mathrm{H}_{33} \mathrm{NO}_{4}$ 446.2302, found 446.2304.

Mp $\left(\mathrm{CHCl}_{3}\right): 71-75{ }^{\circ} \mathrm{C}$. 


\section{References}

1 A. Tourteau, E. Merlet, A. Bontemps, M. Leland, P. Helissey, S. Giorgi-Renault and S. DesbèneFinck, European J. Org. Chem., 2015, 2015, 7028-7035.

2 M. G. Banwell, M. T. Jones, D. T. J. Loong, D. W. Lupton, D. M. Pinkerton, J. K. Ray and A. C. Willis, Tetrahedron, 2010, 66, 9252-9262.

3 D. H. Tuo, Q. He, Q. Q. Wang, Y. F. Ao and D. X. Wang, Chinese J. Chem., 2019, 37, 684688.

$4 \quad$ K. Ando, T. Kobayashi and N. Uchida, Org. Lett., 2015, 17, 2554-2557.

5 G. Zhang, R. X. Bai, C. H. Li, C. G. Feng and G. Q. Lin, Tetrahedron, 2019, 75, 1658-1662.

$6 \quad$ P. Cazeau, F. Duboudin, F. Moulines, O. Babot and J. Dunogues, Tetrahedron, 1987, 43, 20752088.

7 L. D. Elliott, S. Kayal, M. W. George and K. Booker-Milburn, J. Am. Chem. Soc., 2020, 142, 14947-14956.

8 W. von E. Doering, X. Cheng, K. Lee and Z. Lin, J. Am. Chem. Soc., 2002, 124, 11642-11652.

9 T. Chen, L. M. Barton, Y. Lin, J. Tsien, D. Kossler, I. Bastida, S. Asai, C. Bi, J. S. Chen, M. Shan, H. Fang, F. G. Fang, H. Choi, L. Hawkins, T. Qin and P. S. Baran, Nature, 2018, 560, $350-354$. 


\section{NMR Spectra}

${ }^{1} \mathrm{H}$ NMR $\left(400 \mathrm{MHz}, \mathrm{CDCl}_{3}\right)$ of compound $\mathbf{1 a}$ (see procedure)<smiles>O=C1C=C(Br)C(=O)[NH2+]1</smiles>

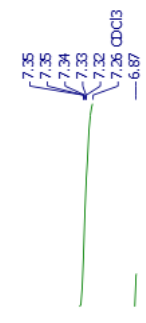

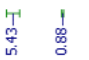

\begin{tabular}{lllllllllllllllllllllllllllll}
3.0 & 12.5 & 12.0 & 11.5 & 11.0 & 10.5 & 10.0 & 9.5 & 9.0 & 8.5 & 8.0 & 7.5 & 7.0 & 6.5 & 6.0 & 1.5 & 1.0 & 4.5 & 4.0 & 3.5 & 3.0 & 2.5 & 2.0 & 1.5 & 1.0 & 0.5 & 0.0 & -1.5 \\
\hline
\end{tabular} ${ }^{13} \mathrm{C}$ NMR (100 MHz, $\mathrm{CDCl}_{3}$ ) of compound $1 \mathbf{a}$ (see procedure)

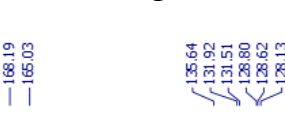<smiles>O=C1C=C(Br)C(=O)[NH2+]1</smiles> 
${ }^{1} \mathrm{H}$ NMR (400 MHz, $\mathrm{CDCl}_{3}$ ) of compound $\mathbf{1 b}$ (see procedure)<smiles>CN1C(=O)C=C(Br)C1=O</smiles>

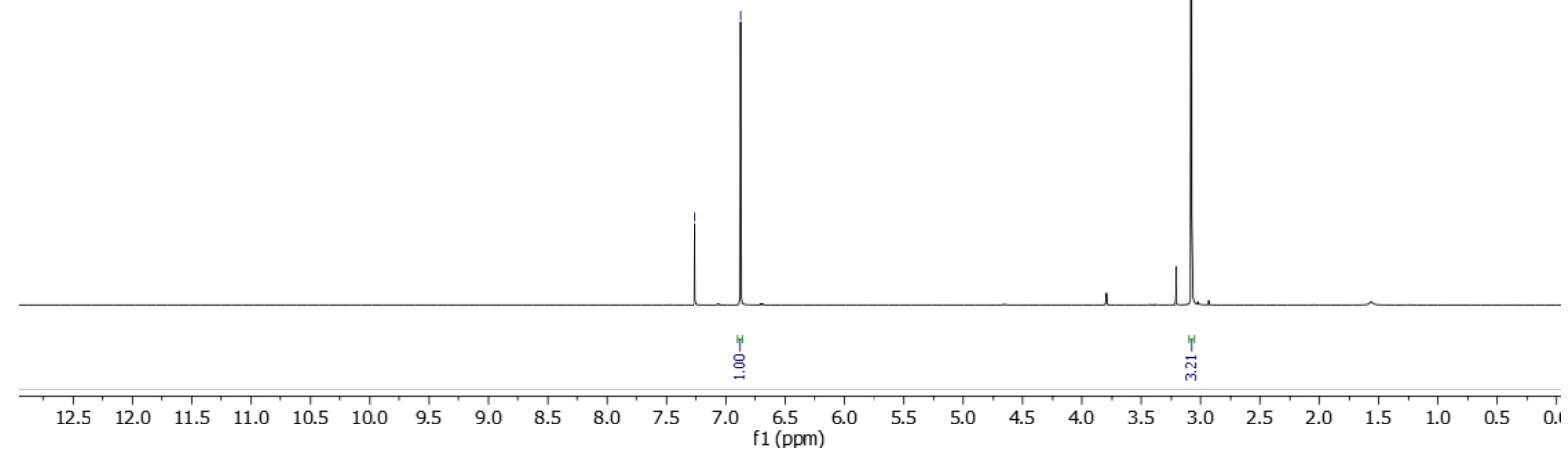

${ }^{13} \mathrm{C}$ NMR $\left(100 \mathrm{MHz}, \mathrm{CDCl}_{3}\right)$ of compound $\mathbf{1 b}$ (see procedure)<smiles>CN1C(=O)C=C(Br)C1=O</smiles>
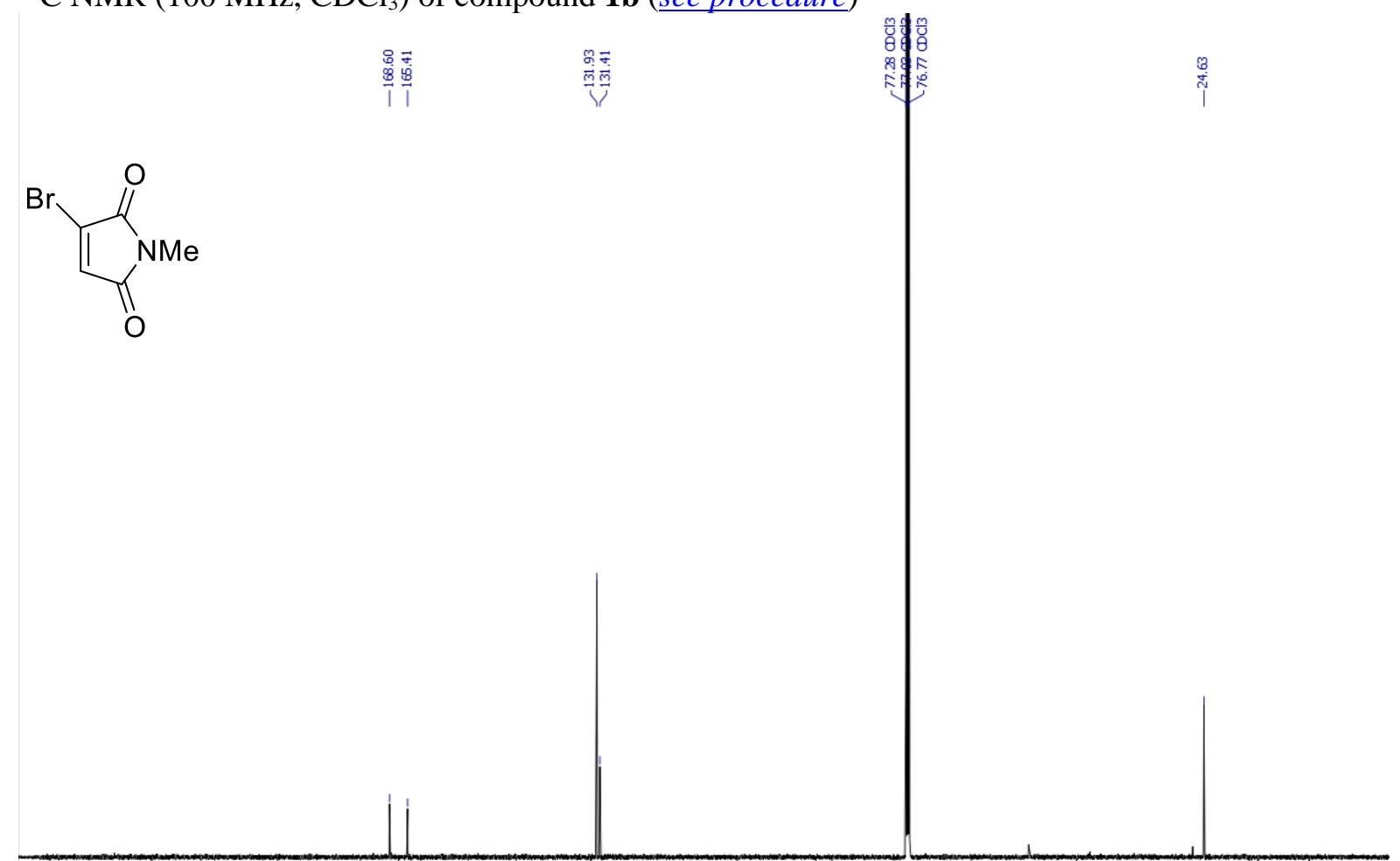

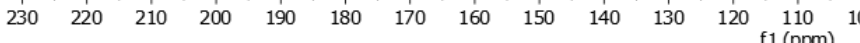


${ }^{1} \mathrm{H}$ NMR (400 MHz, $\mathrm{CDCl}_{3}$ ) of compound $\mathbf{1 c}$ (see procedure)<smiles>O=C1C=C(Br)C(=O)N1</smiles>

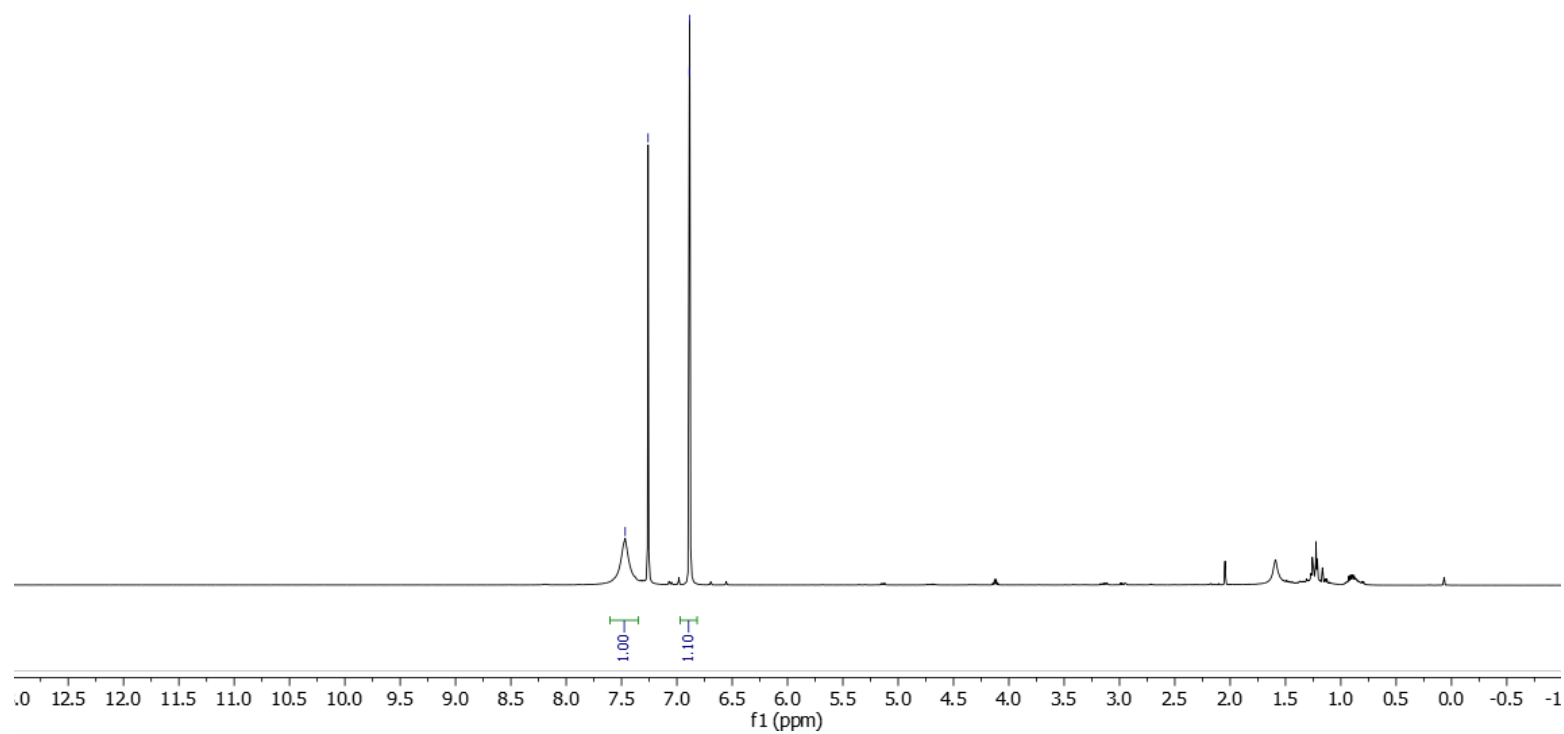

${ }^{13} \mathrm{C}$ NMR (100 MHz, $\left.\mathrm{CDCl}_{3}\right)$ of compound $1 \mathrm{c}$ (see procedure $)$<smiles>O=C1C=C(Br)C(=O)N1</smiles>

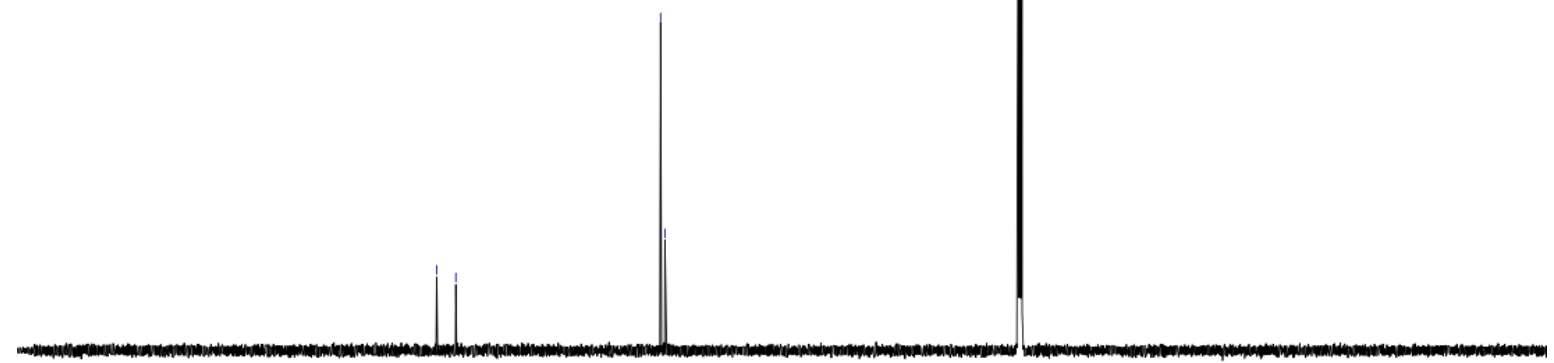

\begin{tabular}{llllllllllllll}
\hline 230 & 220 & 210 & 200 & 190 & 180 & 170 & 160 & 150 & 140 & 130 & 120 & 110 & 100
\end{tabular} 
${ }^{1} \mathrm{H}$ NMR (400 MHz, $\mathrm{CDCl}_{3}$ ) of compound $\mathbf{2 a}$ (see procedure)

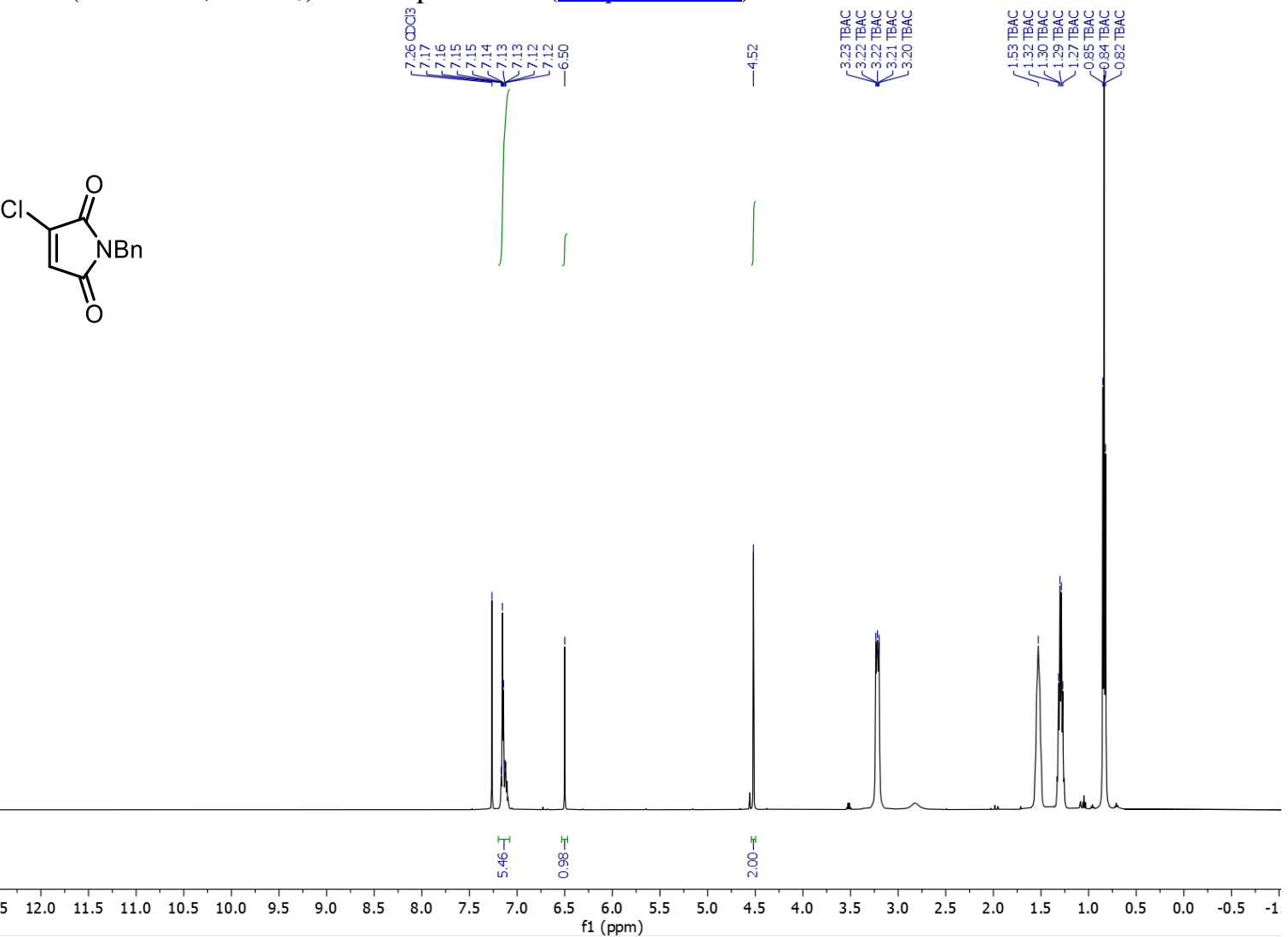

${ }^{13} \mathrm{C} \mathrm{NMR}\left(100 \mathrm{MHz}, \mathrm{CDCl}_{3}\right)$ of compound $\mathbf{2 a}$ (see procedure)

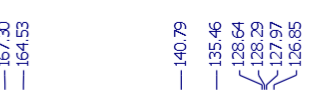<smiles></smiles>

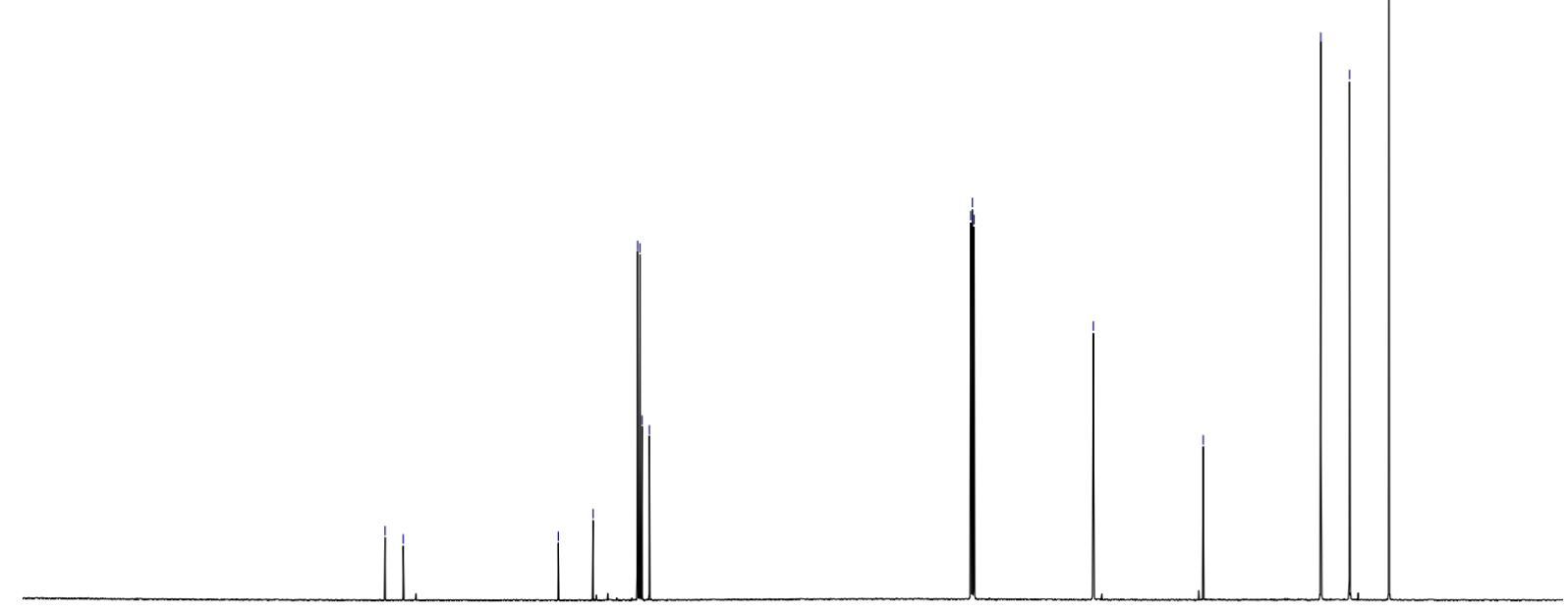

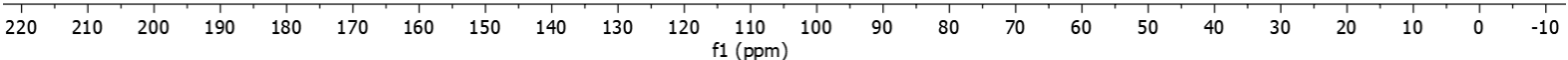


${ }^{1} \mathrm{H}$ NMR (400 MHz, $\mathrm{CDCl}_{3}$ ) of compound $\mathbf{2 b}$ (see procedure)<smiles>CNC(=O)C1C(=O)C=C(Cl)C1=O</smiles>

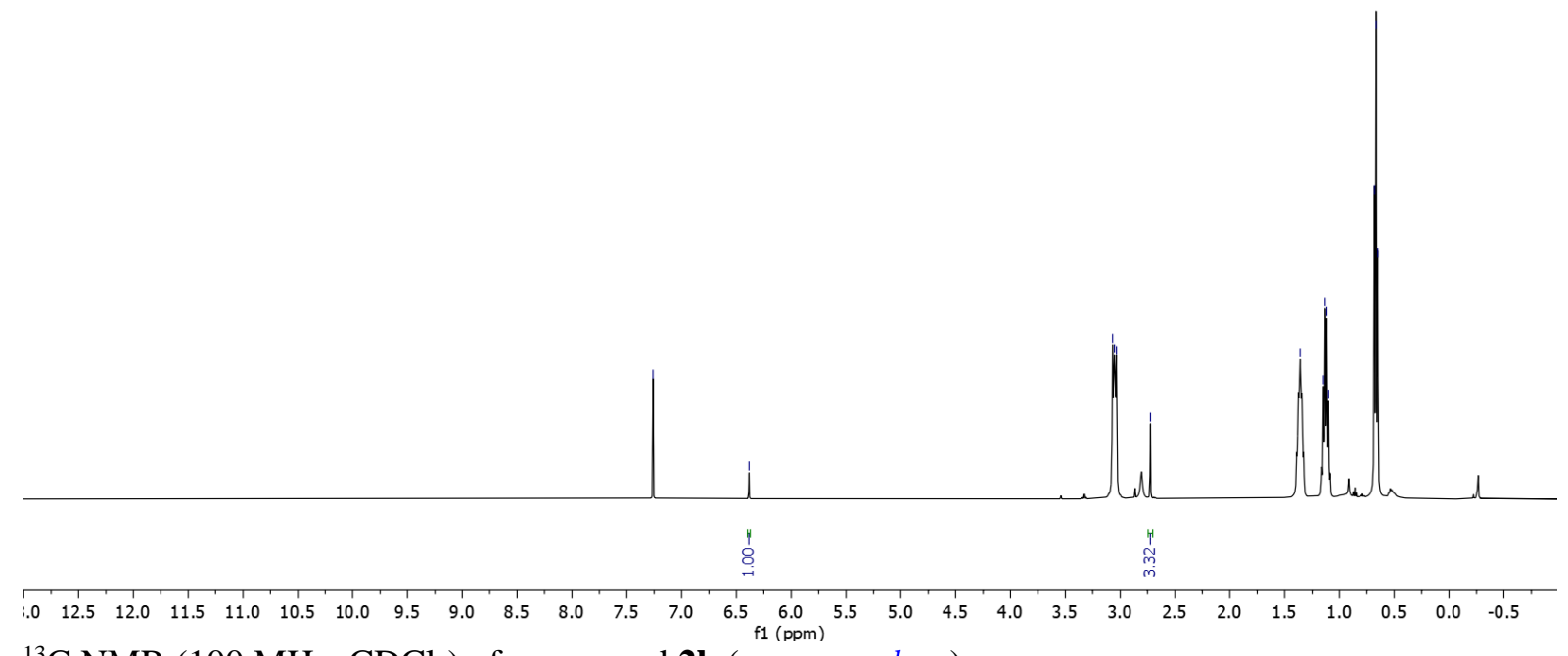

${ }^{13} \mathrm{C}$ NMR (100 MHz, $\mathrm{CDCl}_{3}$ ) of compound $\mathbf{2 b}$ (see procedure)

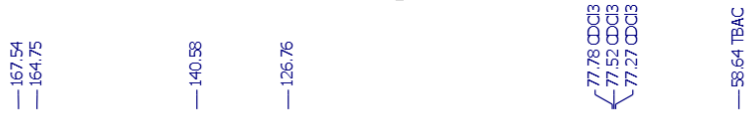<smiles>CN1C(=O)C=C(Cl)C1=O</smiles>

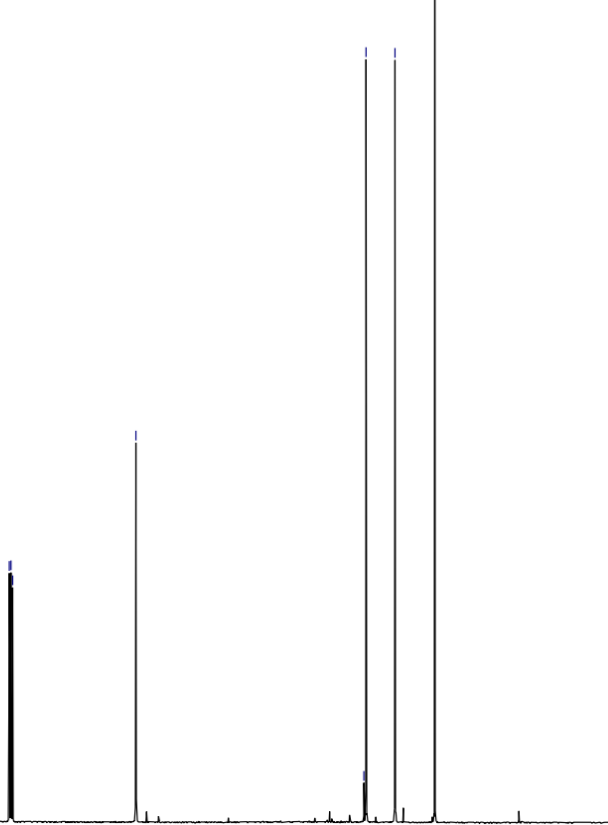

$\begin{array}{lllllllllllllllllllllllllllll}220 & 210 & 200 & 190 & 180 & 170 & 160 & 150 & 140 & 130 & 120 & 110 & 100 & 90 & 80 & 70 & 60 & 50 & 40 & 30 & 20 & 10 & 0 & -10\end{array}$ 
${ }^{1} \mathrm{H}$ NMR (400 MHz, $\mathrm{CDCl}_{3}$ ) of compound $\mathbf{2 c}$ (see procedure)

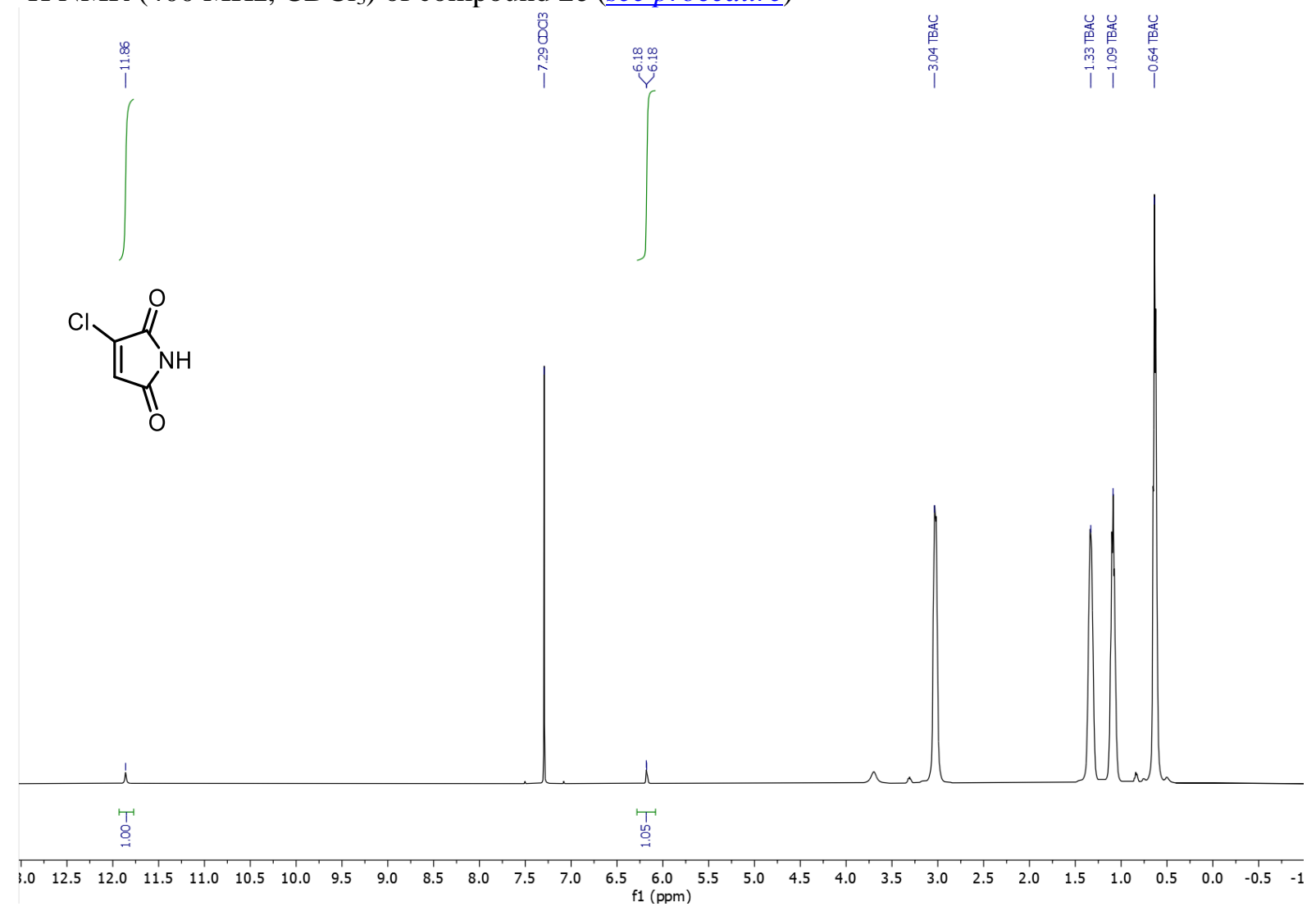

${ }^{13} \mathrm{C}$ NMR (100 MHz, $\left.\mathrm{CDCl}_{3}\right)$ of compound $2 \mathrm{c}$ (see procedure)

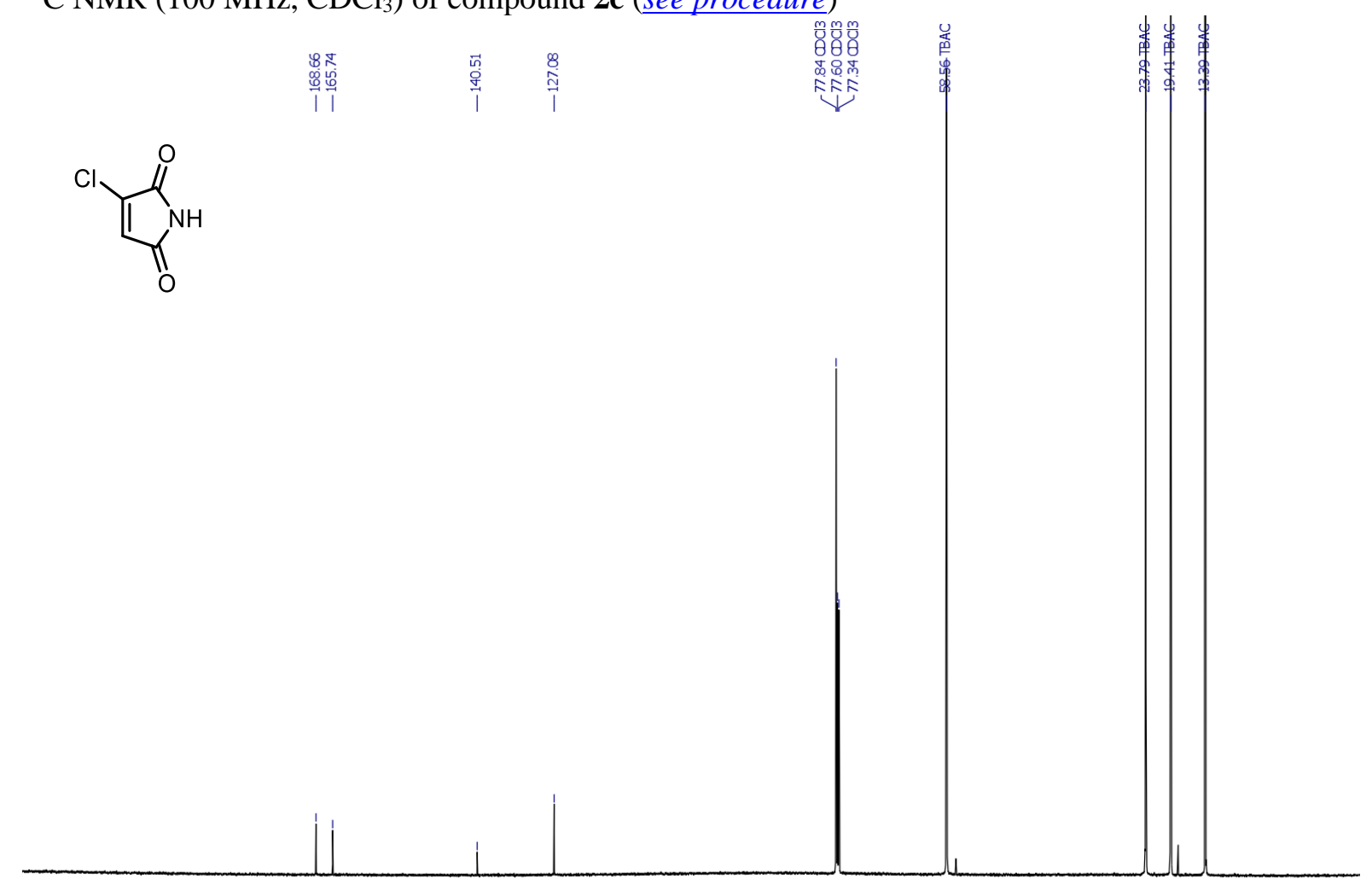

\begin{tabular}{lllllllllllllllllllllllll}
\hline 0 & 210 & 200 & 190 & 180 & 170 & 160 & 150 & 140 & 130 & 120 & 110 & 100 & 90 & 80 & 70 & 60 & 50 & 40 & 30 & 20 & 10 & 0 & -10
\end{tabular} 
${ }^{1} \mathrm{H}$ NMR (400 MHz, $\mathrm{CDCl}_{3}$ ) of compound $\mathbf{S 1}$ (see procedure)

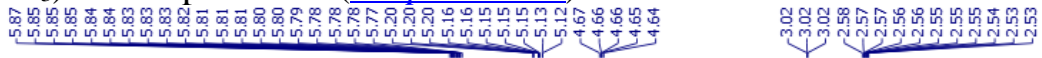<smiles>C=CCCOC1C(=O)N(C)C(=O)C1Cl</smiles><smiles>CC1CCCCC1</smiles><smiles>CCC1C=CCC1</smiles><smiles>CC1CC1C</smiles>

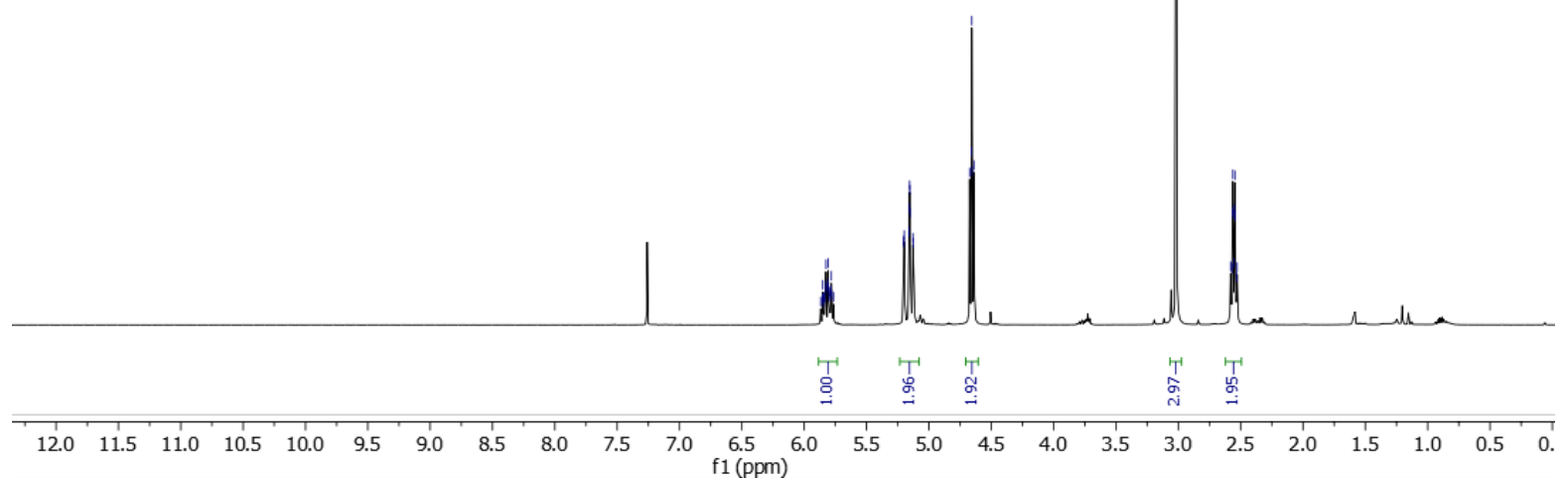

${ }^{13} \mathrm{C}$ NMR $\left(100 \mathrm{MHz}, \mathrm{CDCl}_{3}\right)$ of compound $\mathbf{S 1}$ (see procedure)<smiles>C=CCCOC1=C(Cl)C(=O)N(C)C1=O</smiles> 
${ }^{1} \mathrm{H}$ NMR (400 MHz, $\mathrm{CDCl}_{3}$ ) of compound $\mathbf{3}$ (see procedure)

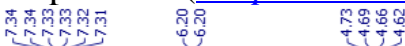

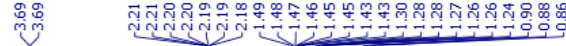<smiles>[13CH3]N1C(=O)[C@H]2C=C(Br)[C@H]2C1=O</smiles>

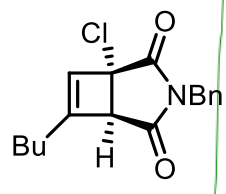

$15: 1$

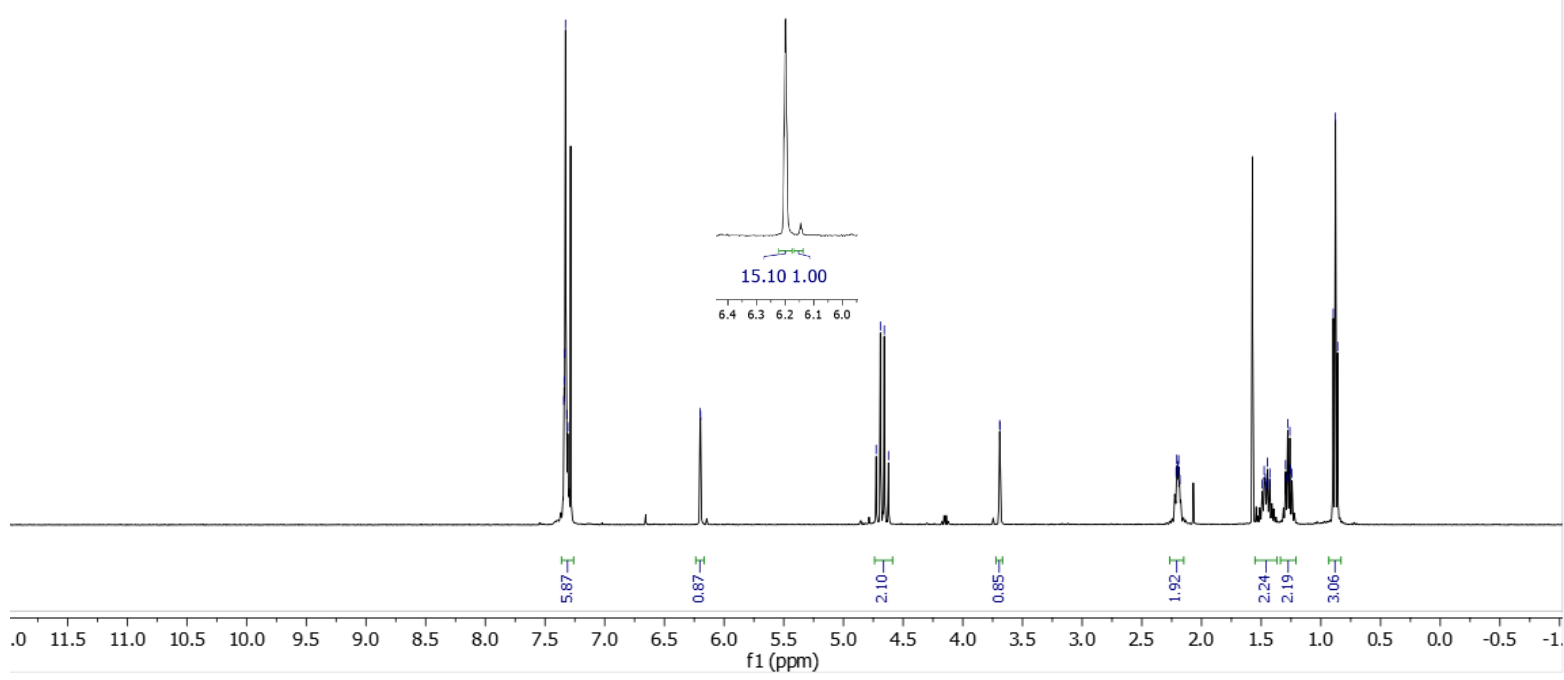

${ }^{13} \mathrm{C}$ NMR $\left(100 \mathrm{MHz}, \mathrm{CDCl}_{3}\right.$ ) of compound 3 (see procedure)

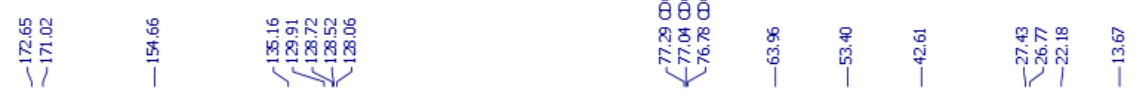<smiles>O=C1[C@H]2C=C(Br)C[C@H]2C(=O)N1Br</smiles><smiles>O=C1C(Br)=C[C@@H]2C(=O)N(Br)C(=O)[C@H]12</smiles>

$15: 1$

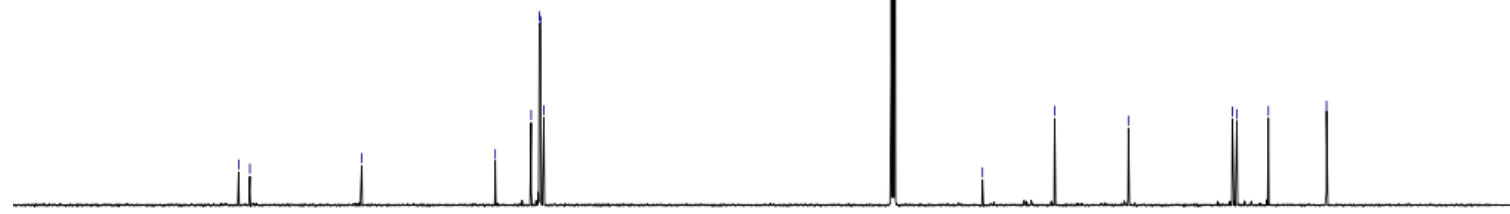

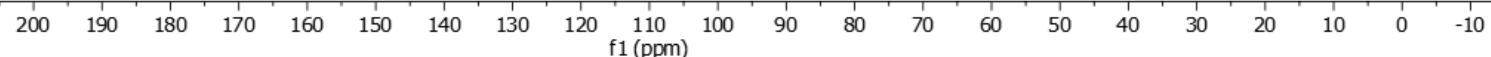


${ }^{1} \mathrm{H}$ NMR (400 MHz, $\mathrm{CDCl}_{3}$ ) of compound 4 (see procedure)

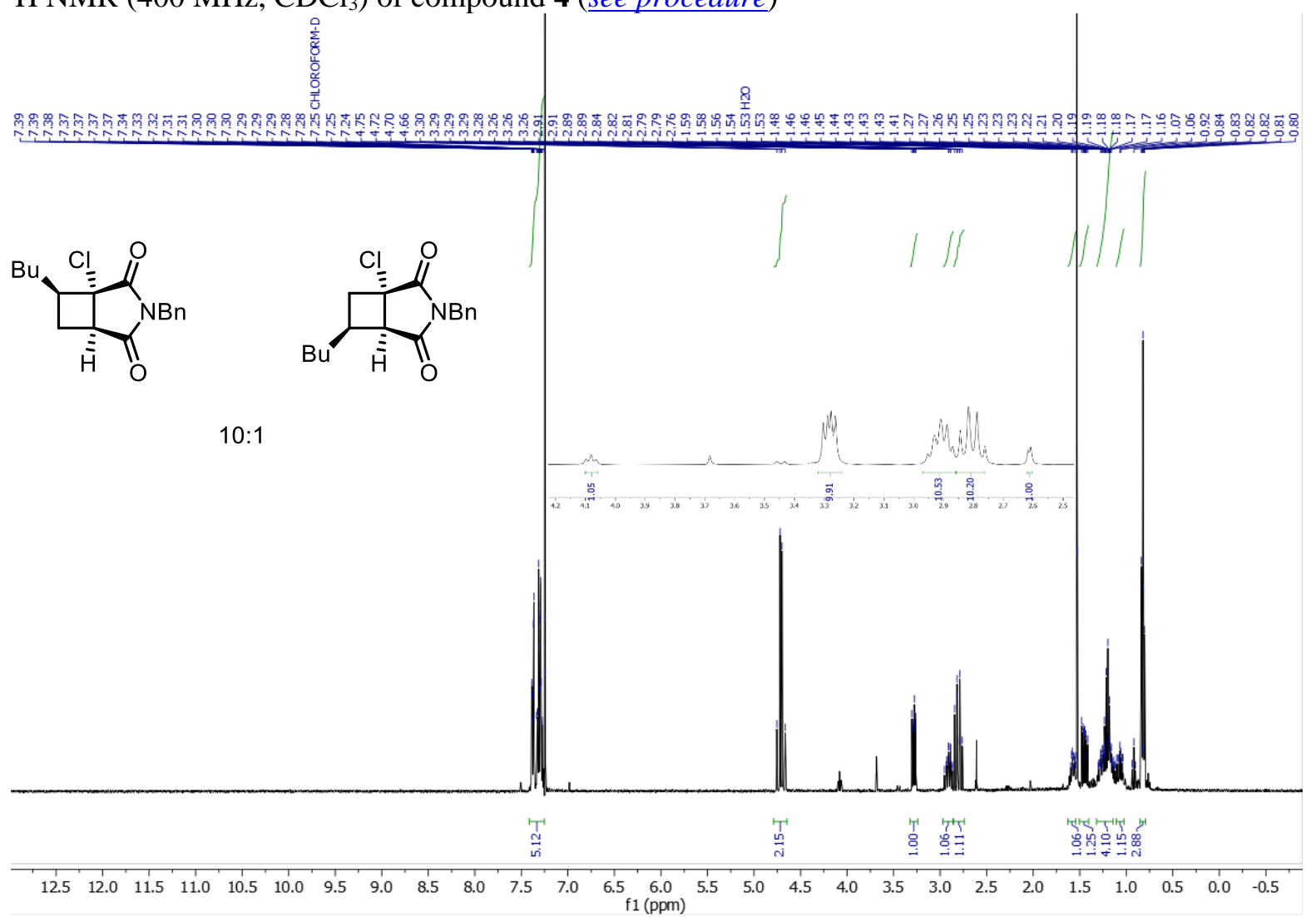

${ }^{13} \mathrm{C}$ NMR $\left(100 \mathrm{MHz}, \mathrm{CDCl}_{3}\right.$ ) of compound 4 (see procedure)
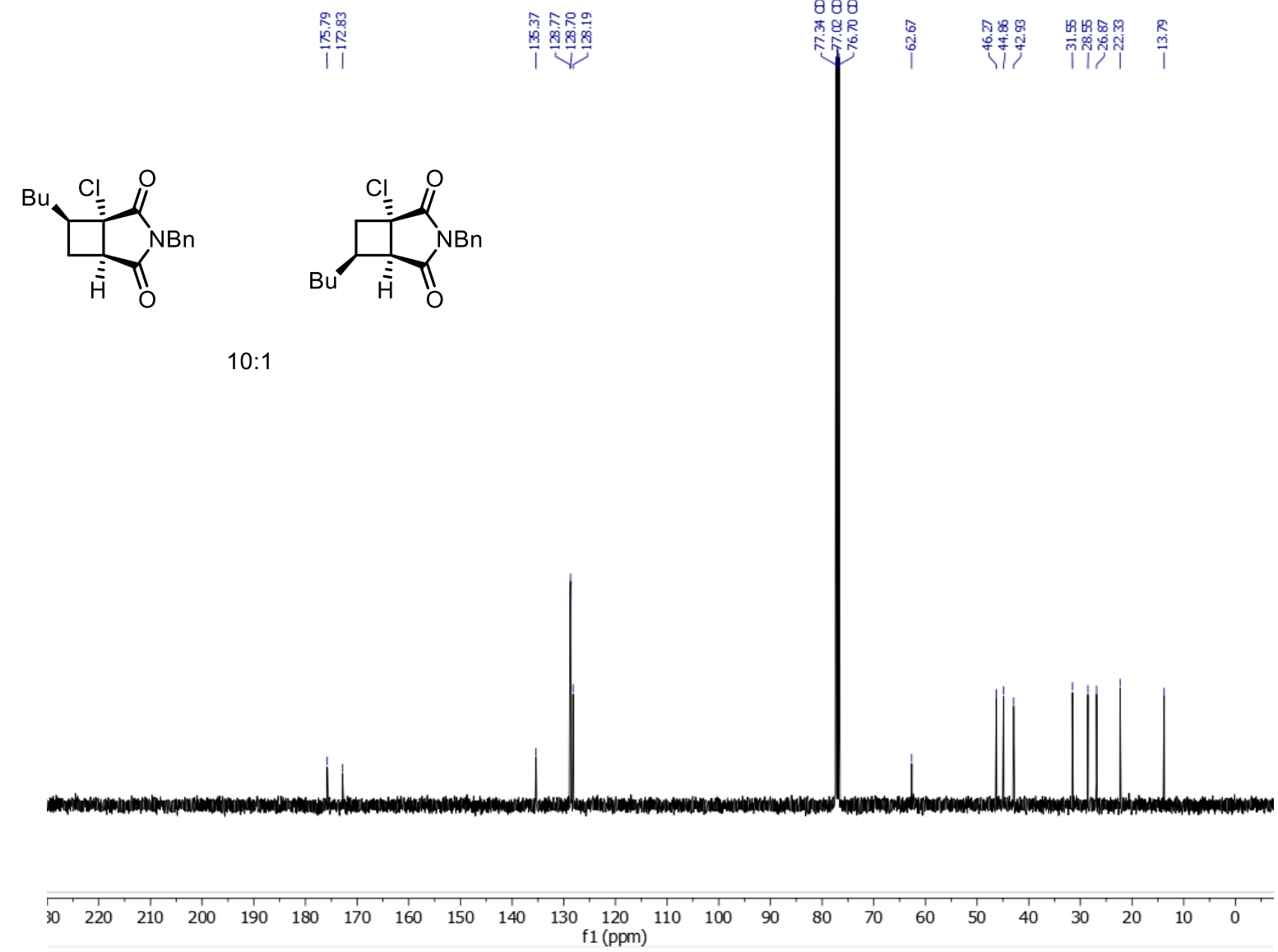
NOE NMR (500 MHz, $\mathrm{CDCl}_{3}$ ) of compound 4 (see procedure)
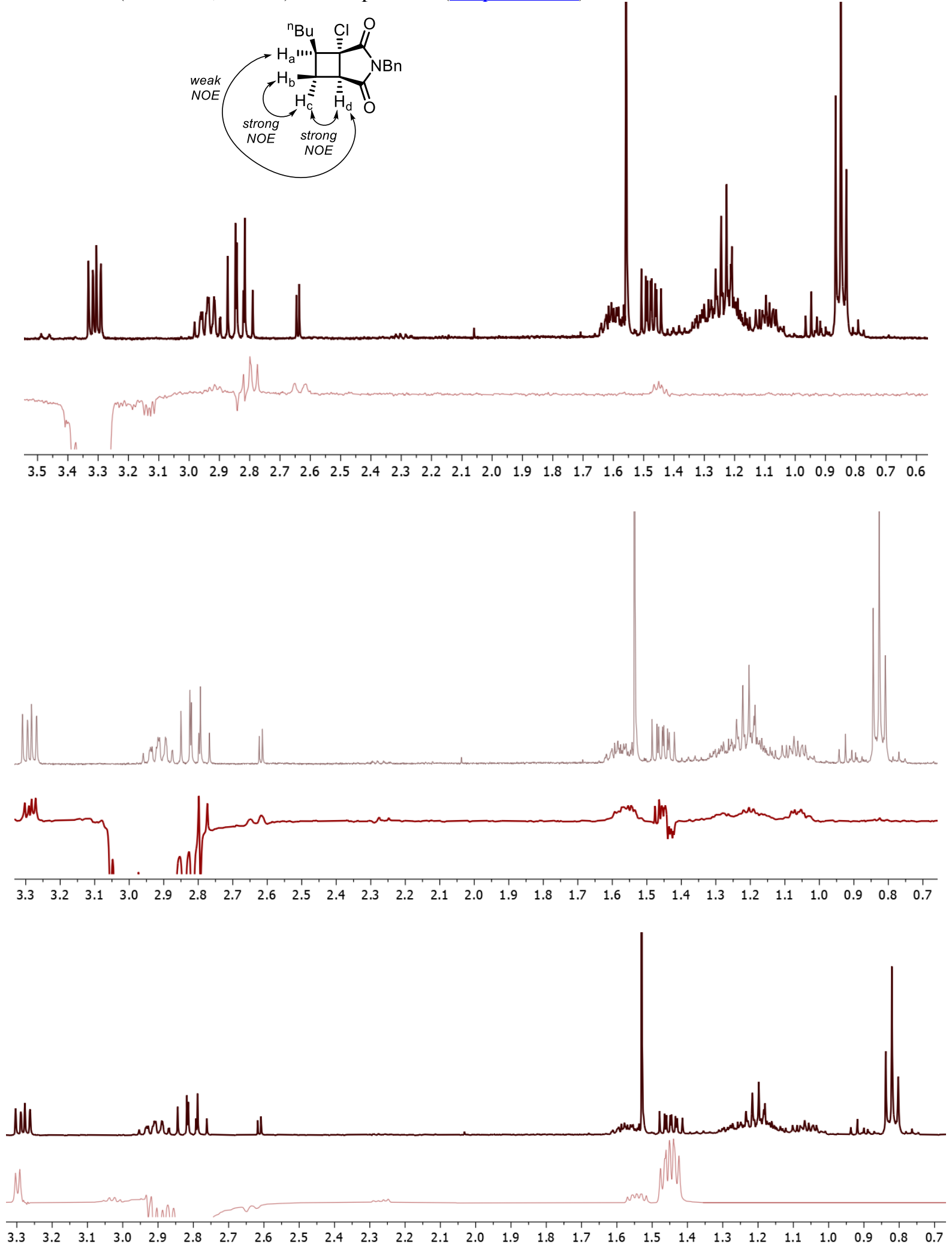
${ }^{1} \mathrm{H} \mathrm{NMR}\left(400 \mathrm{MHz}, \mathrm{CDCl}_{3}\right.$ ) of compound 5 (see procedure)

觉

B.
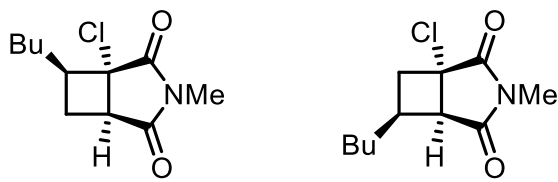

$12: 1$
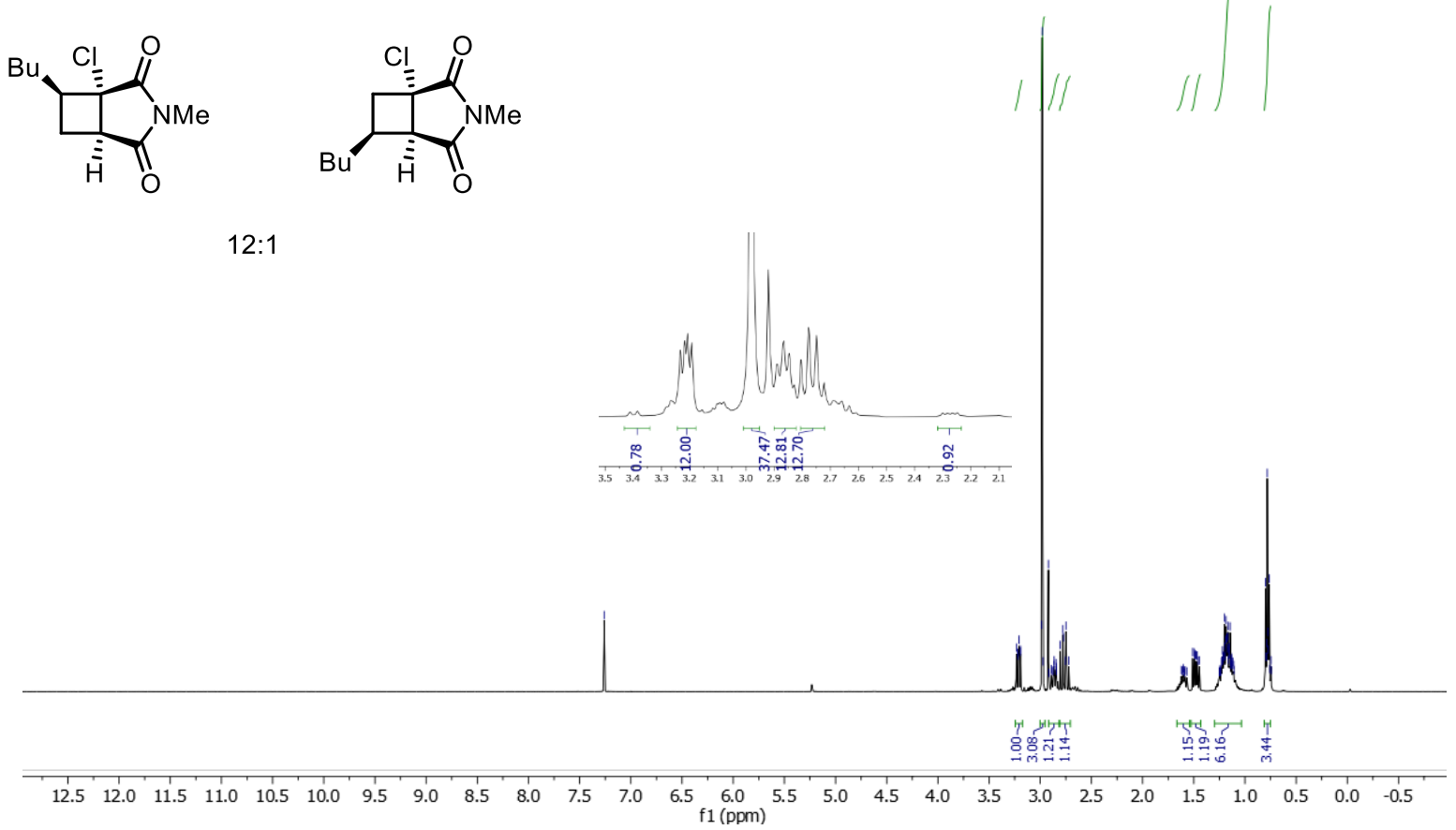

${ }^{13} \mathrm{C}$ NMR $\left(100 \mathrm{MHz}, \mathrm{CDCl}_{3}\right)$ of compound $\mathbf{5}$ (see procedure)<smiles>C1=C[As]2C=C12</smiles>

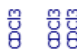

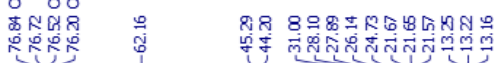

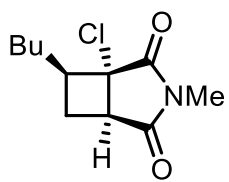<smiles>CN1C(=O)C2C(Cl)CC(Br)C2C1=O</smiles>

$12: 1$

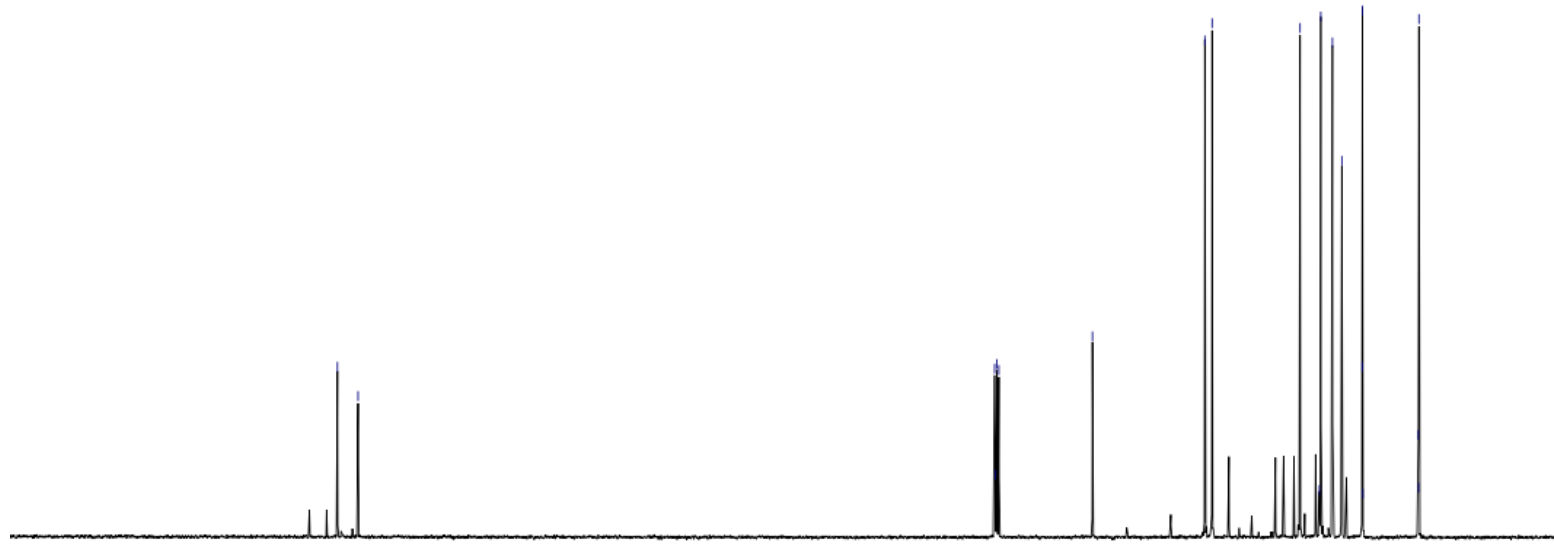

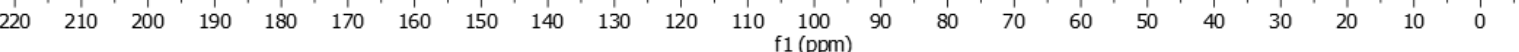


${ }^{1} \mathrm{H}$ NMR $\left(400 \mathrm{MHz}, \mathrm{CDCl}_{3}\right.$ ) of compound 6 (see procedure)

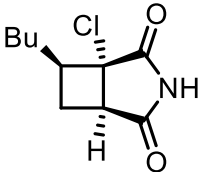<smiles>O=C1NC(=O)C2C(Br)CC1C2Br</smiles>

$11: 1$
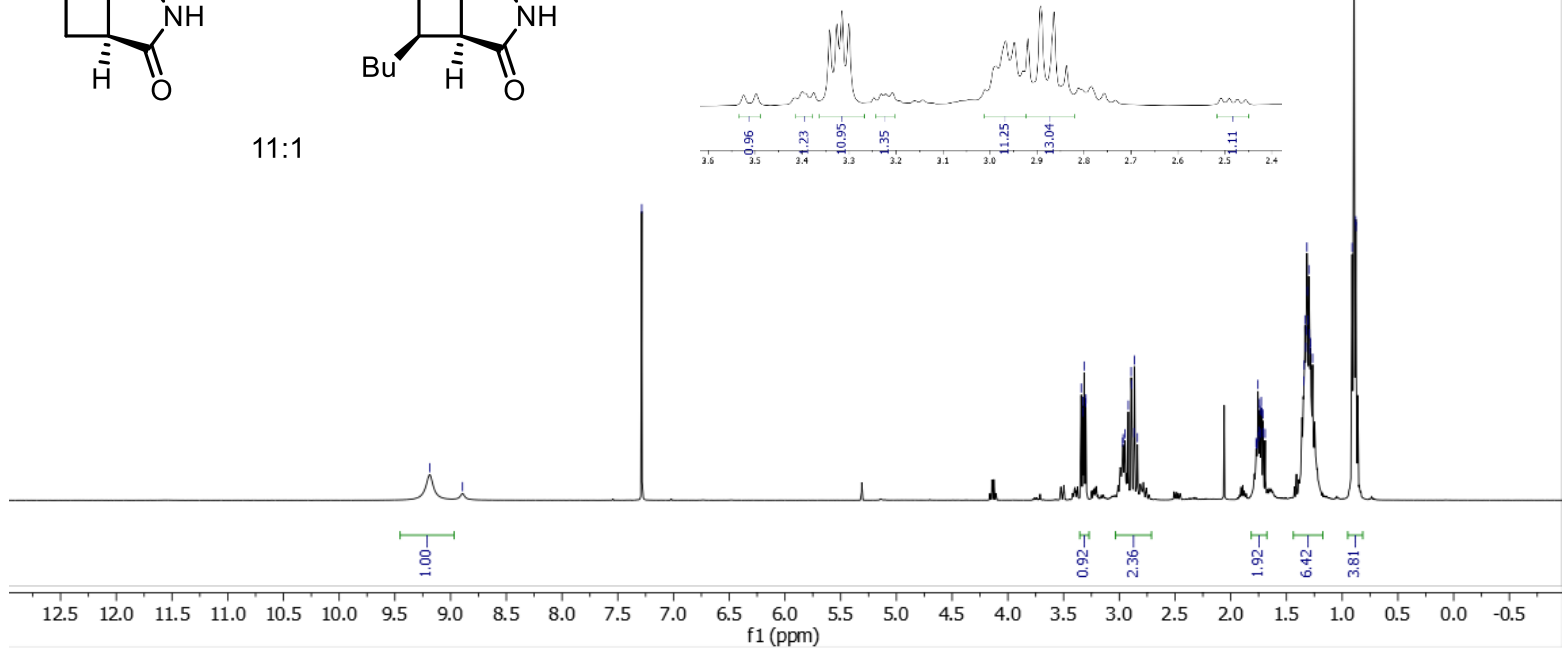

${ }^{13} \mathrm{C} \mathrm{NMR}\left(100 \mathrm{MHz}, \mathrm{CDCl}_{3}\right)$ of compound 6 (see procedure)<smiles>C1=C[AsH]1</smiles>

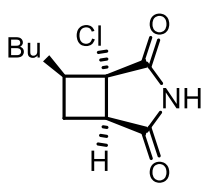<smiles>O=C1NC(=O)C2C(Cl)CC1C2Br</smiles>

$11: 1$
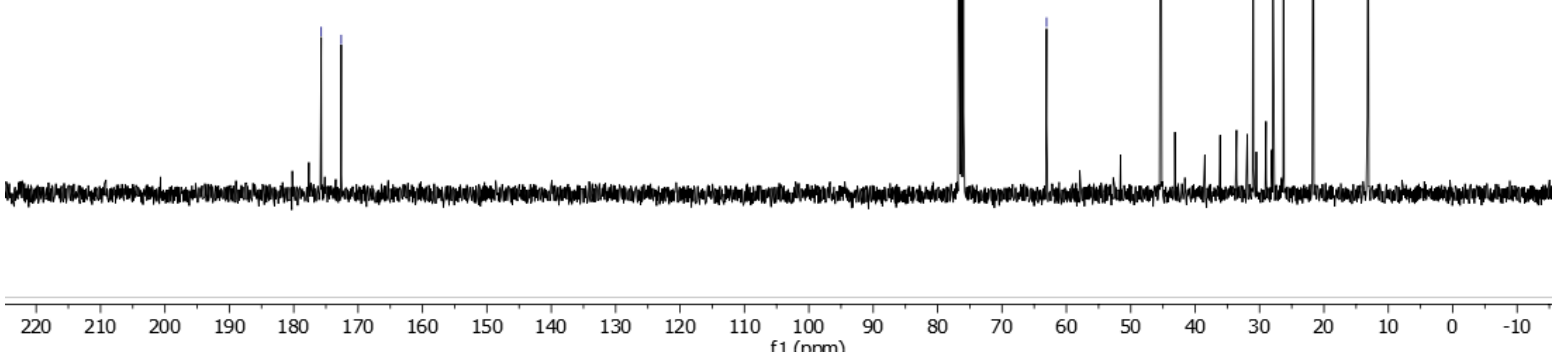
${ }^{1} \mathrm{H}$ NMR (400 MHz, $\mathrm{CDCl}_{3}$ ) of compound 7 (see procedure)
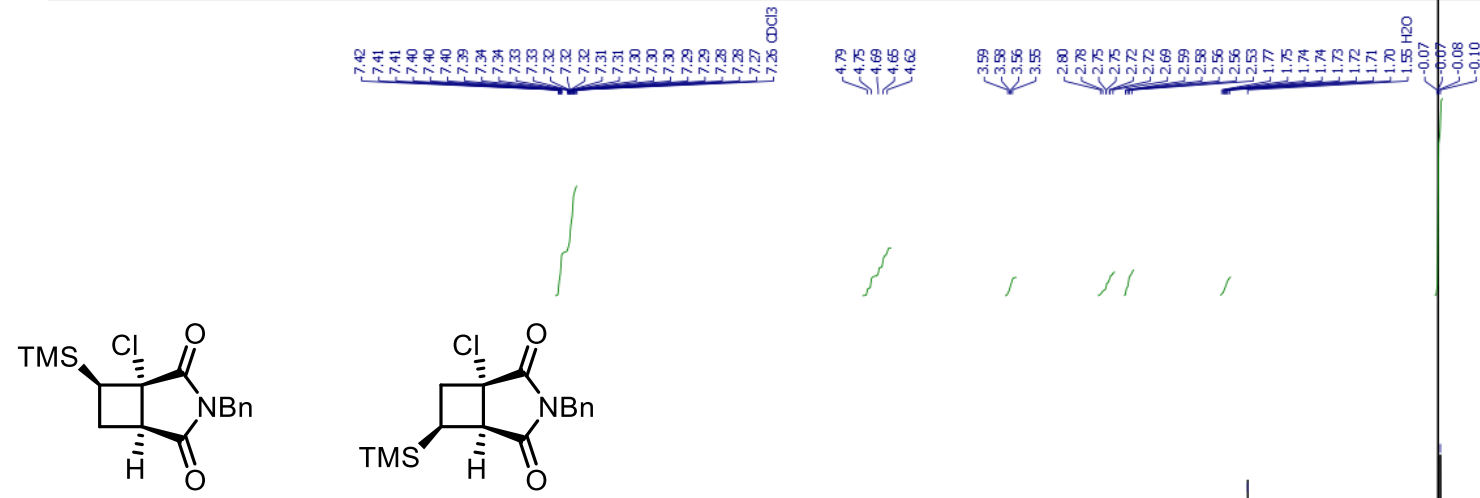

$4: 1$

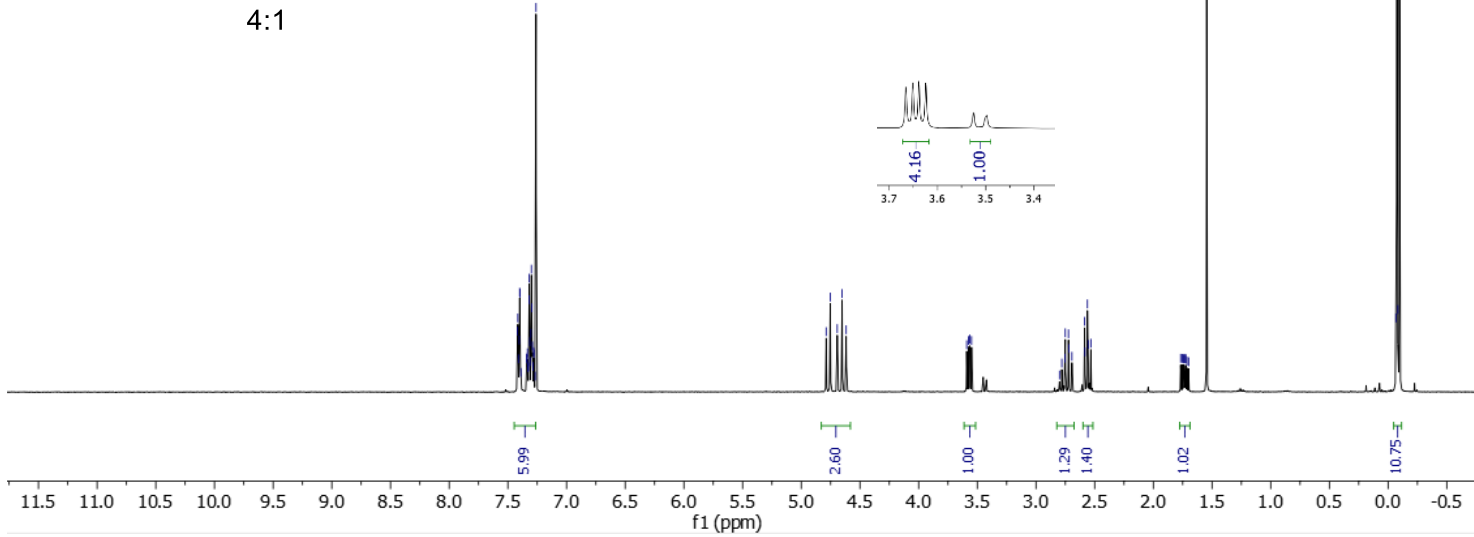

${ }^{13} \mathrm{C} \mathrm{NMR}\left(100 \mathrm{MHz}, \mathrm{CDCl}_{3}\right.$ ) of compound 7 (see procedure)

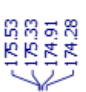

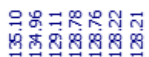
(1)
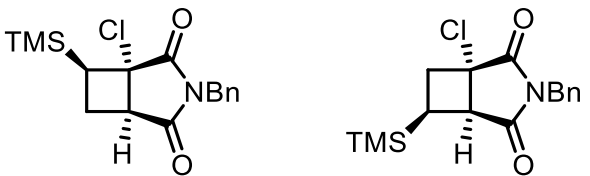

$4: 1$

\begin{tabular}{lllllllllllllllllllllllllll}
\hline 230 & 220 & 210 & 200 & 190 & 180 & 170 & 160 & 150 & 140 & 130 & 120 & 110 & 100 & 90 & 80 & 70 & 60 & 50 & 40 & 30 & 20 & 10 & 0 & -10
\end{tabular} 
${ }^{1} \mathrm{H}$ NMR (400 MHz, $\mathrm{CDCl}_{3}$ ) of compound 8 (see procedure)

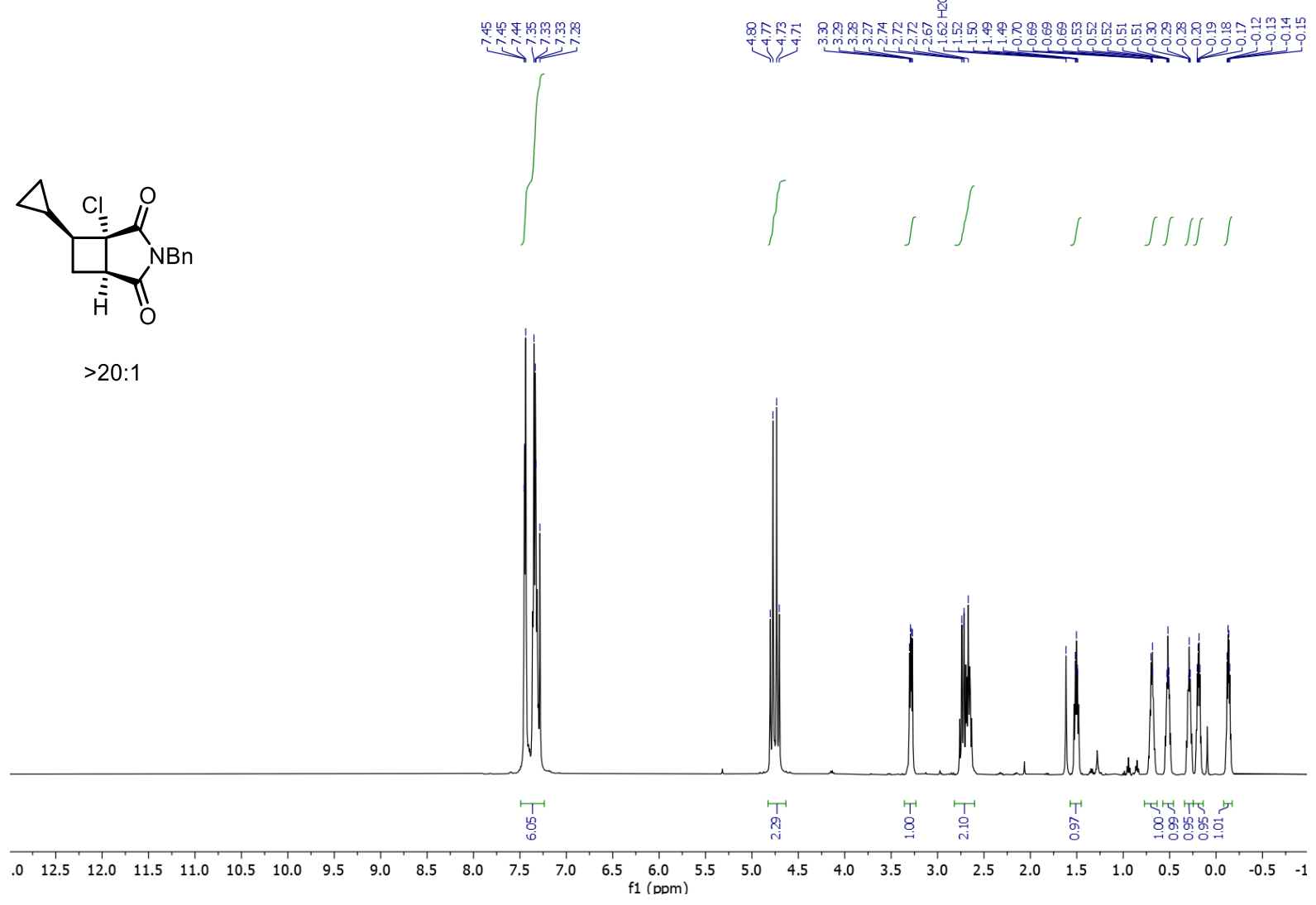

${ }^{13} \mathrm{C}$ NMR $\left(100 \mathrm{MHz}, \mathrm{CDCl}_{3}\right.$ ) of compound $\mathbf{8}$ (see procedure)
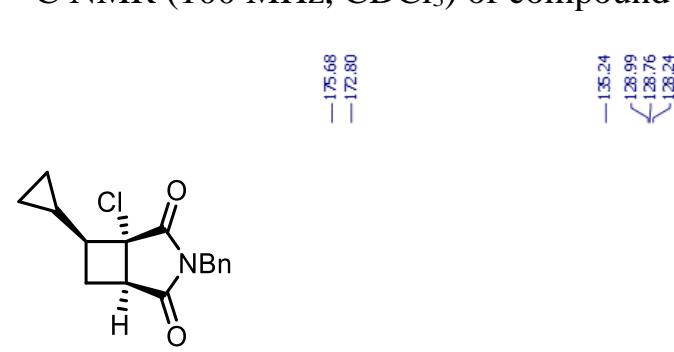

$>20: 1$

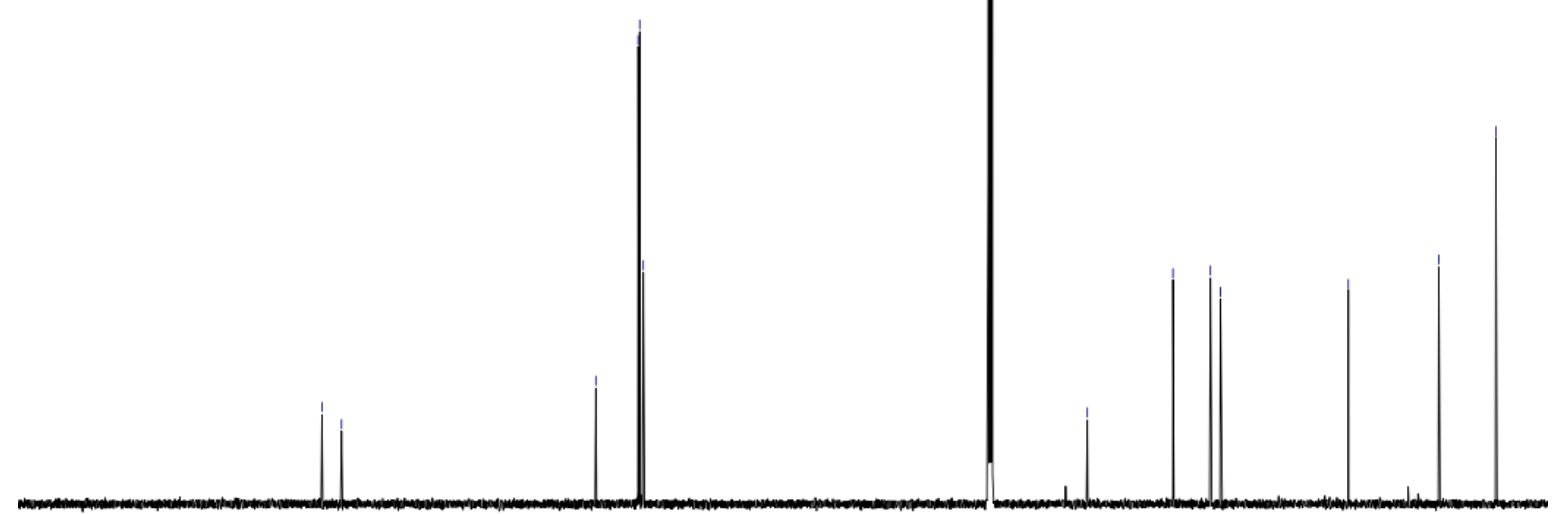

\begin{tabular}{|l|llllllllllllllllllllllllll}
\hline 20 & 210 & 200 & 190 & 180 & 170 & 160 & 150 & 140 & 130 & 120 & 110 & 100 & 90 & 80 & 70 & 60 & 50 & 40 & 30 & 20 & 10 & 0
\end{tabular} 
${ }^{1} \mathrm{H}$ NMR (400 MHz, $\mathrm{CDCl}_{3}$ ) of compound 9 (see procedure)

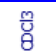

7
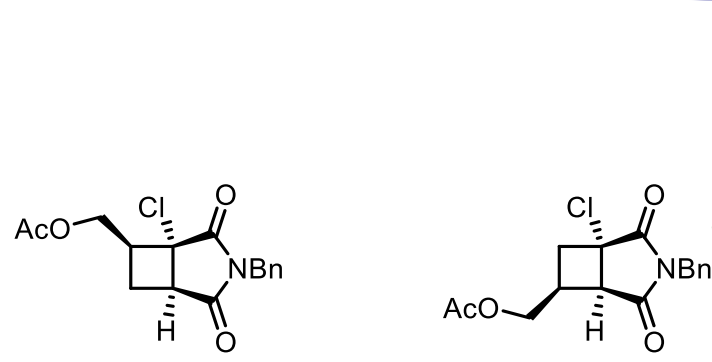

$4: 1$
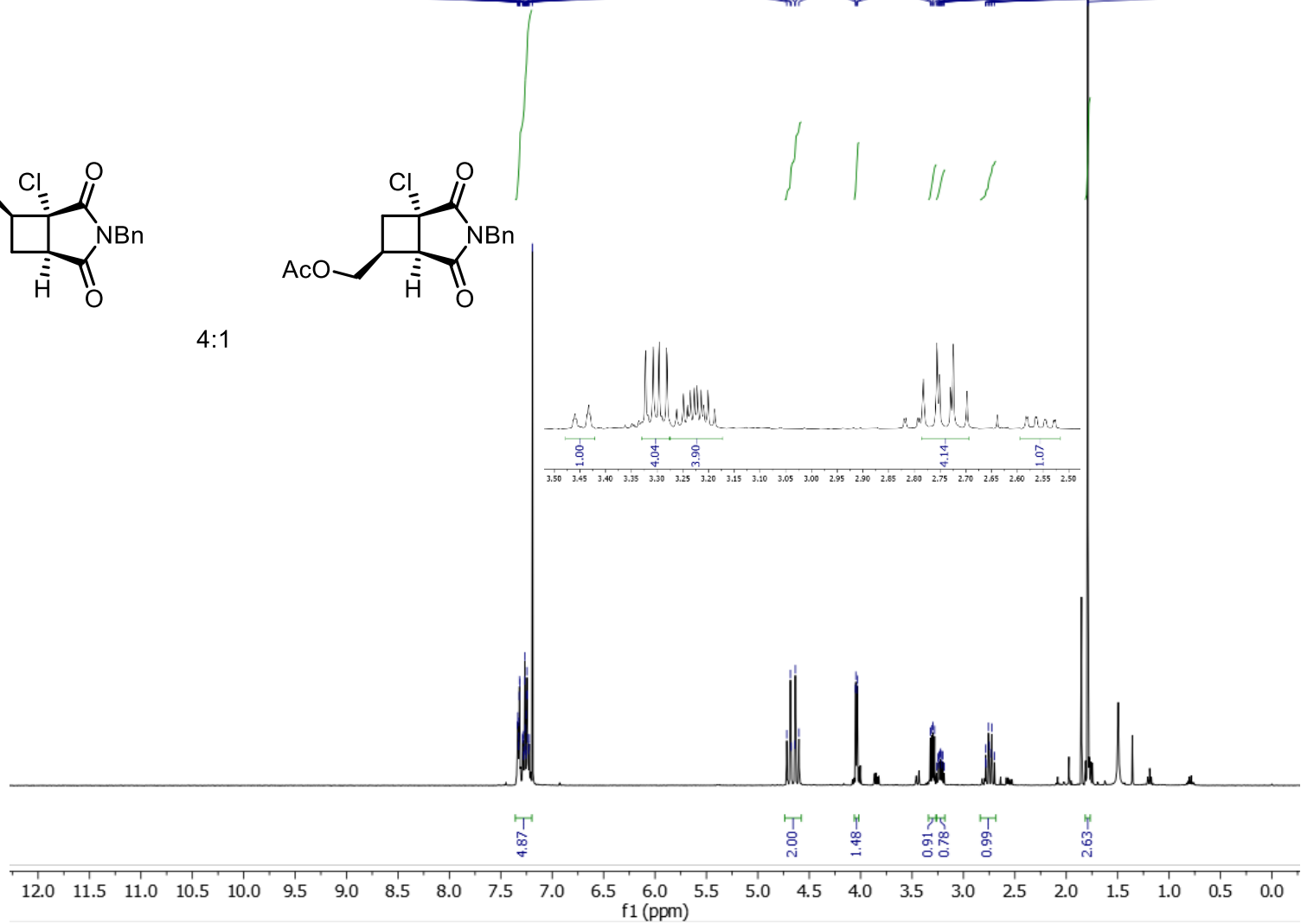

${ }^{13} \mathrm{C}$ NMR (100 MHz, $\mathrm{CDCl}_{3}$ ) of compound 9 (see procedure)

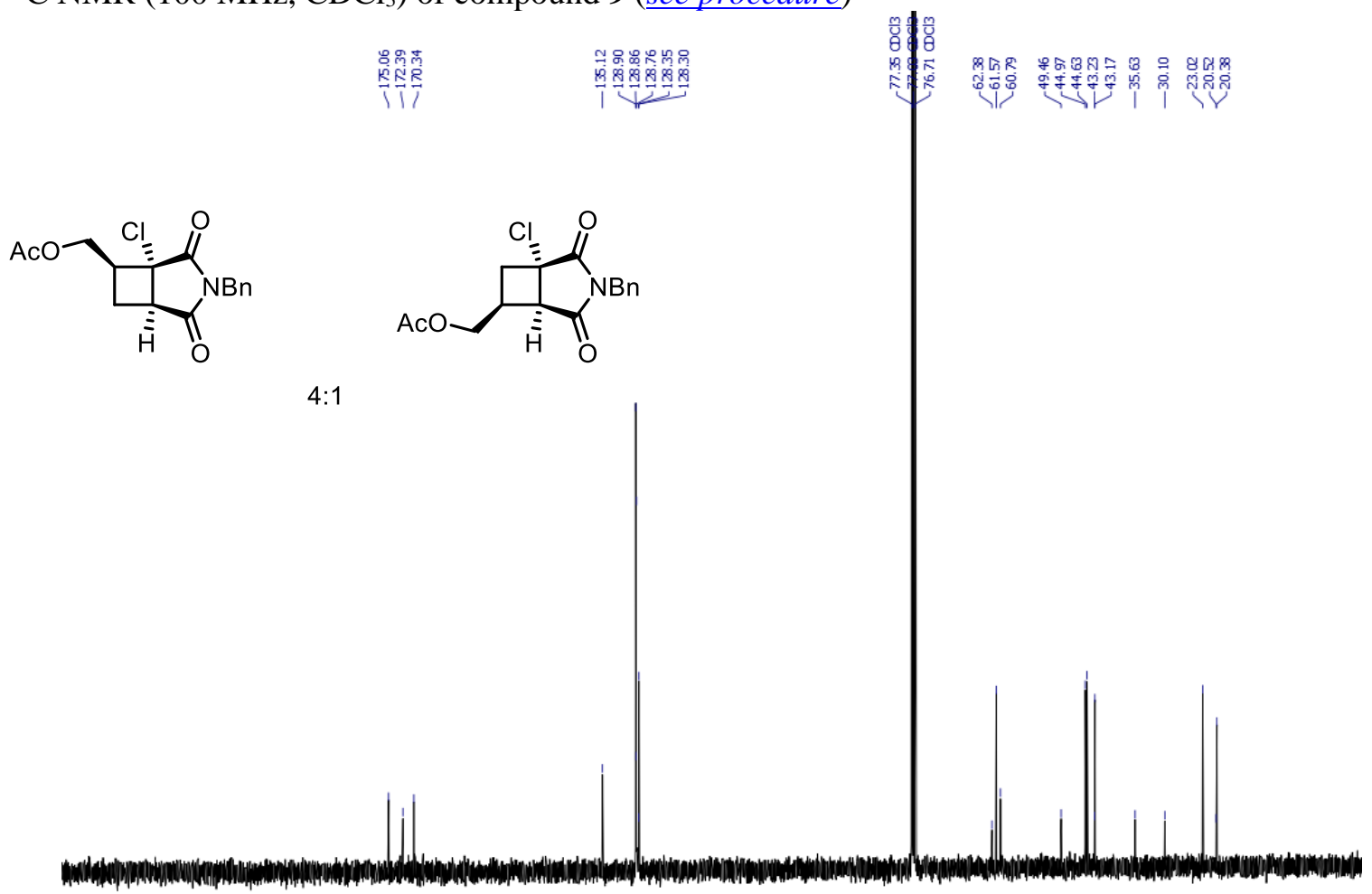

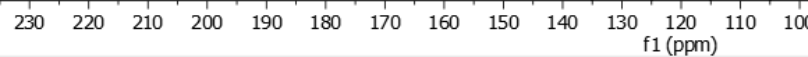


${ }^{1} \mathrm{H}$ NMR (400 MHz, $\mathrm{CDCl}_{3}$ ) of compound $\mathbf{1 0}$ (see procedure)

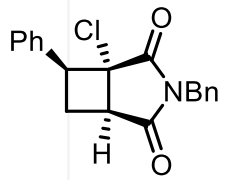

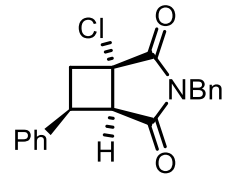

$7: 1$

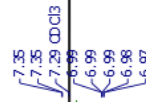

R8 58 ल 8 8.

옹

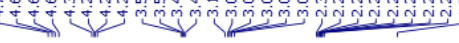

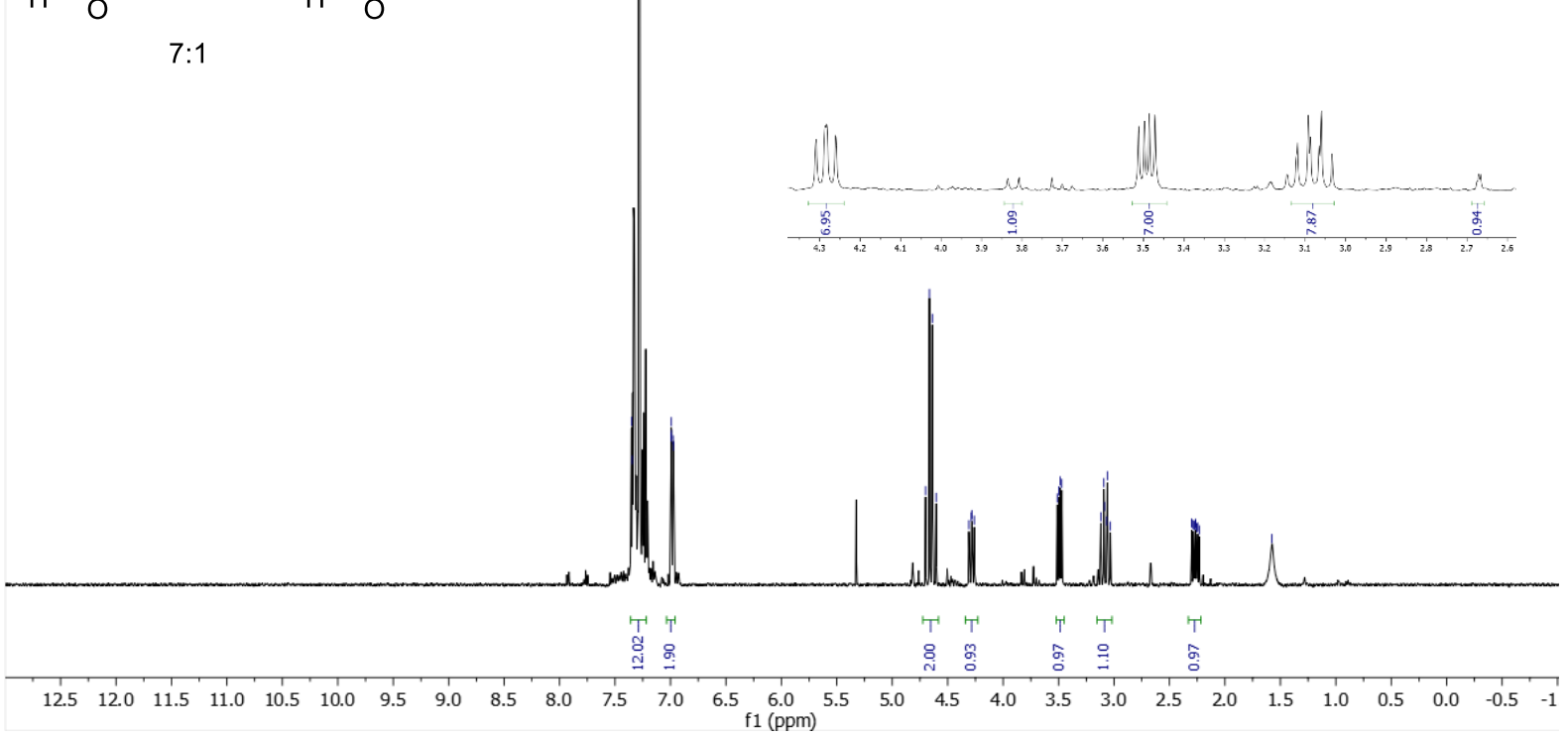

${ }^{13} \mathrm{C}$ NMR $\left(100 \mathrm{MHz}, \mathrm{CDCl}_{3}\right)$ of compound $\mathbf{1 0}$ (see procedure)

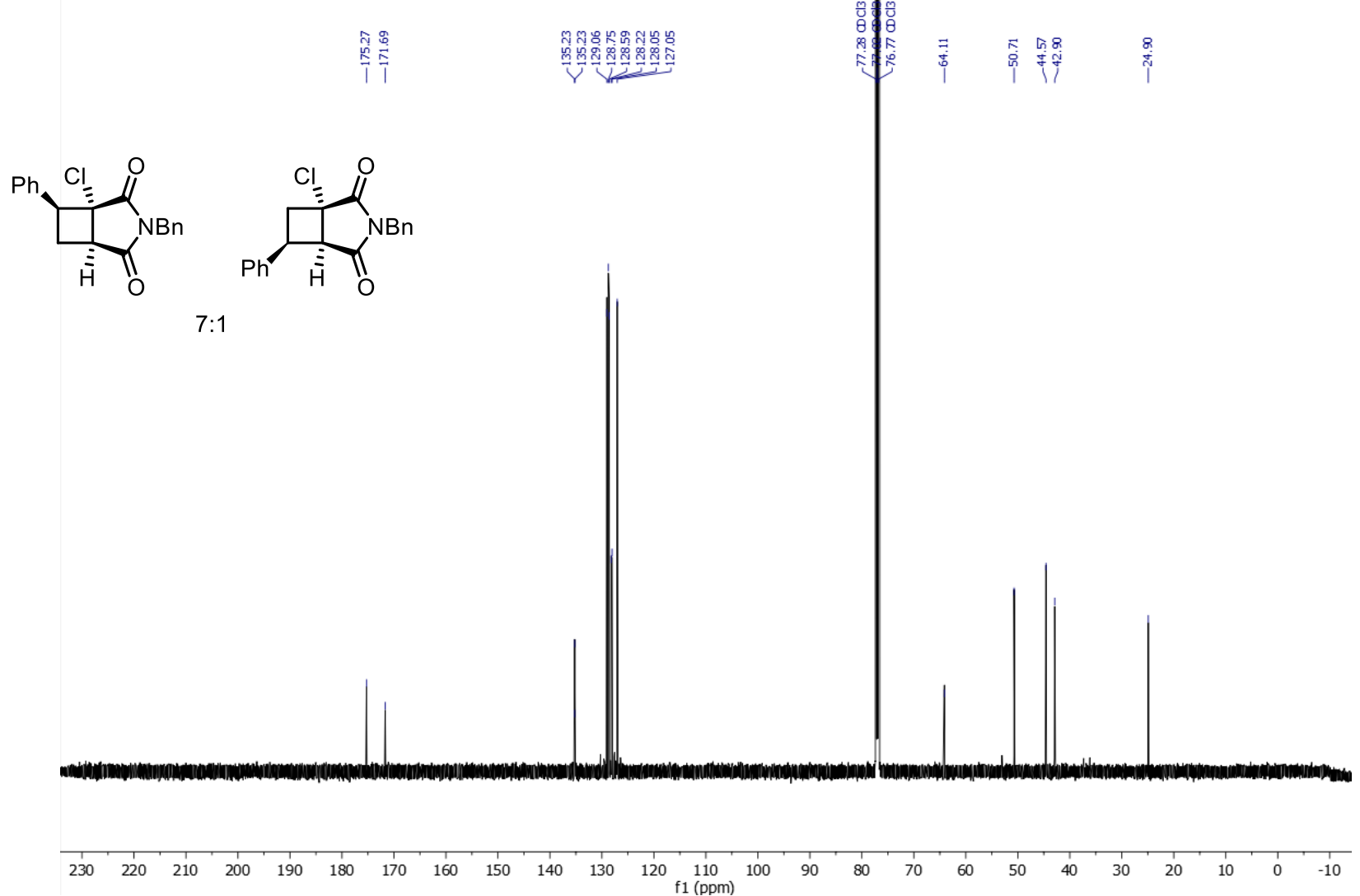


${ }^{1} \mathrm{H}$ NMR (400 MHz, $\mathrm{CDCl}_{3}$ ) of compound 11 (see procedure)

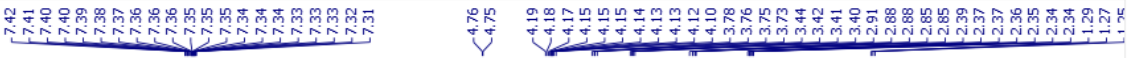
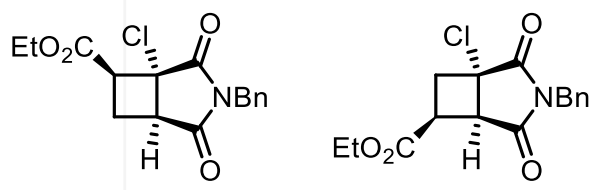

$4: 1$

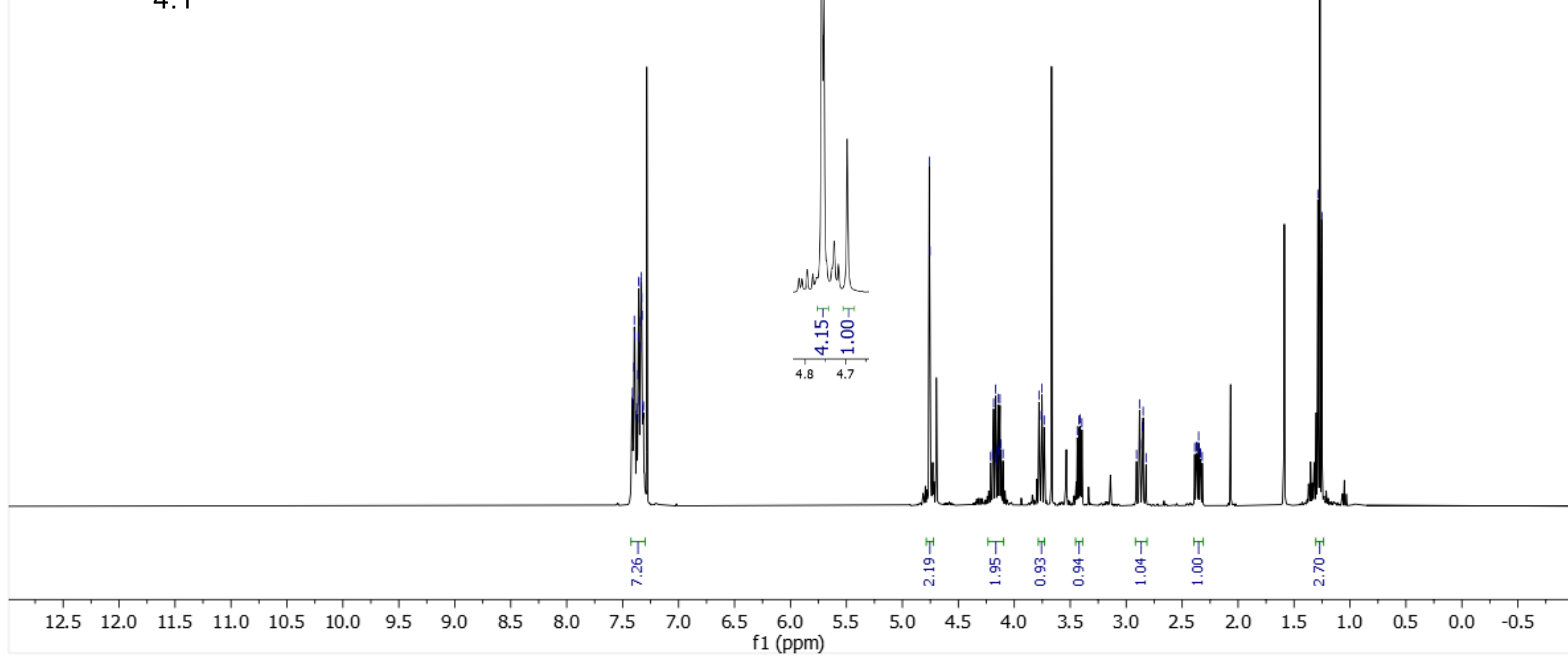

${ }^{13} \mathrm{C}$ NMR $\left(100 \mathrm{MHz}, \mathrm{CDCl}_{3}\right.$ ) of compound 11 (see procedure)

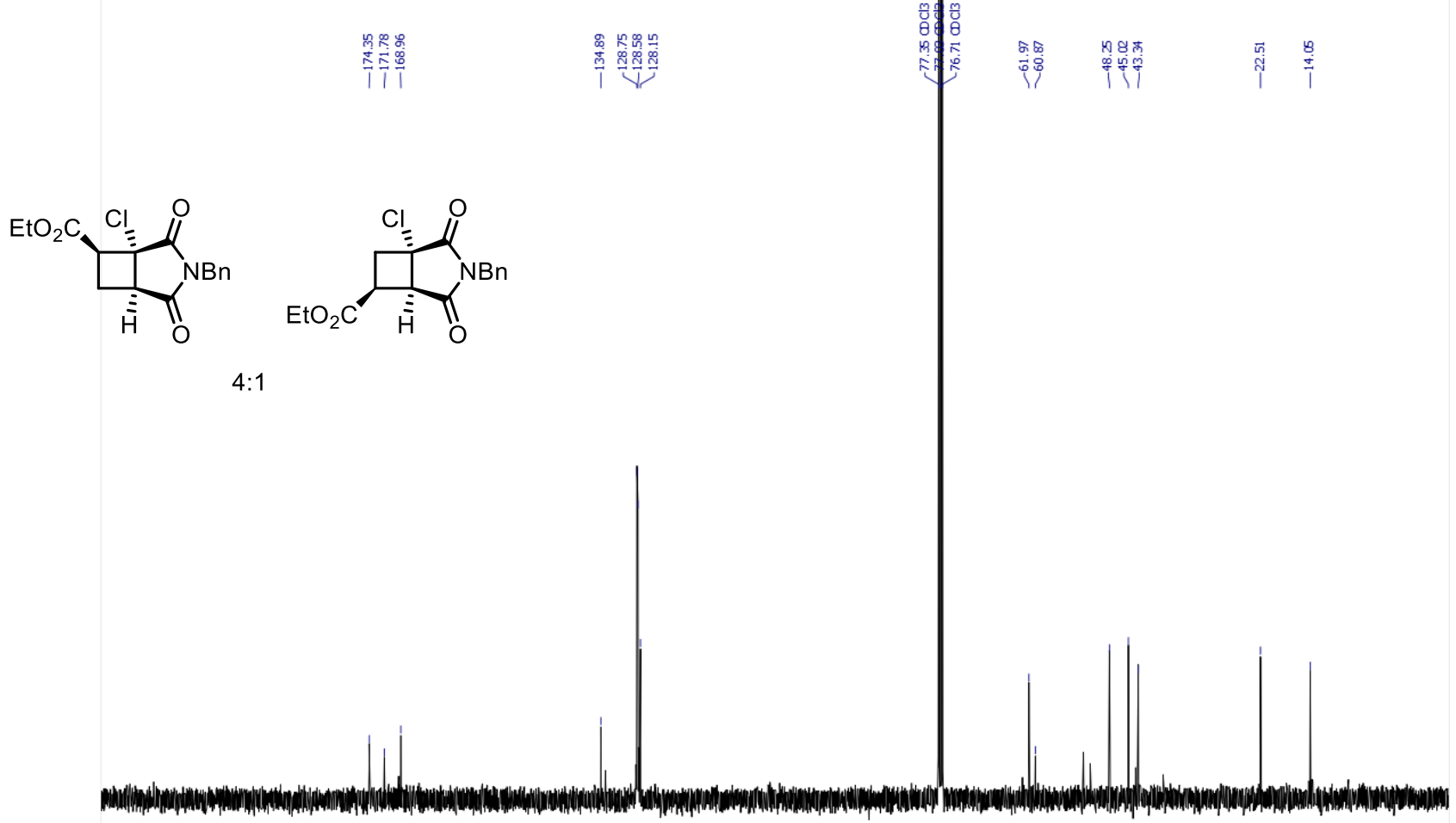

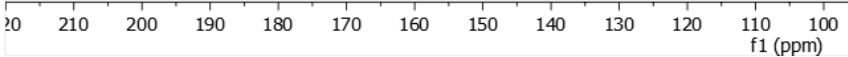


${ }^{1} \mathrm{H}$ NMR (400 MHz, $\mathrm{CDCl}_{3}$ ) of compound 12 (see procedure) 要

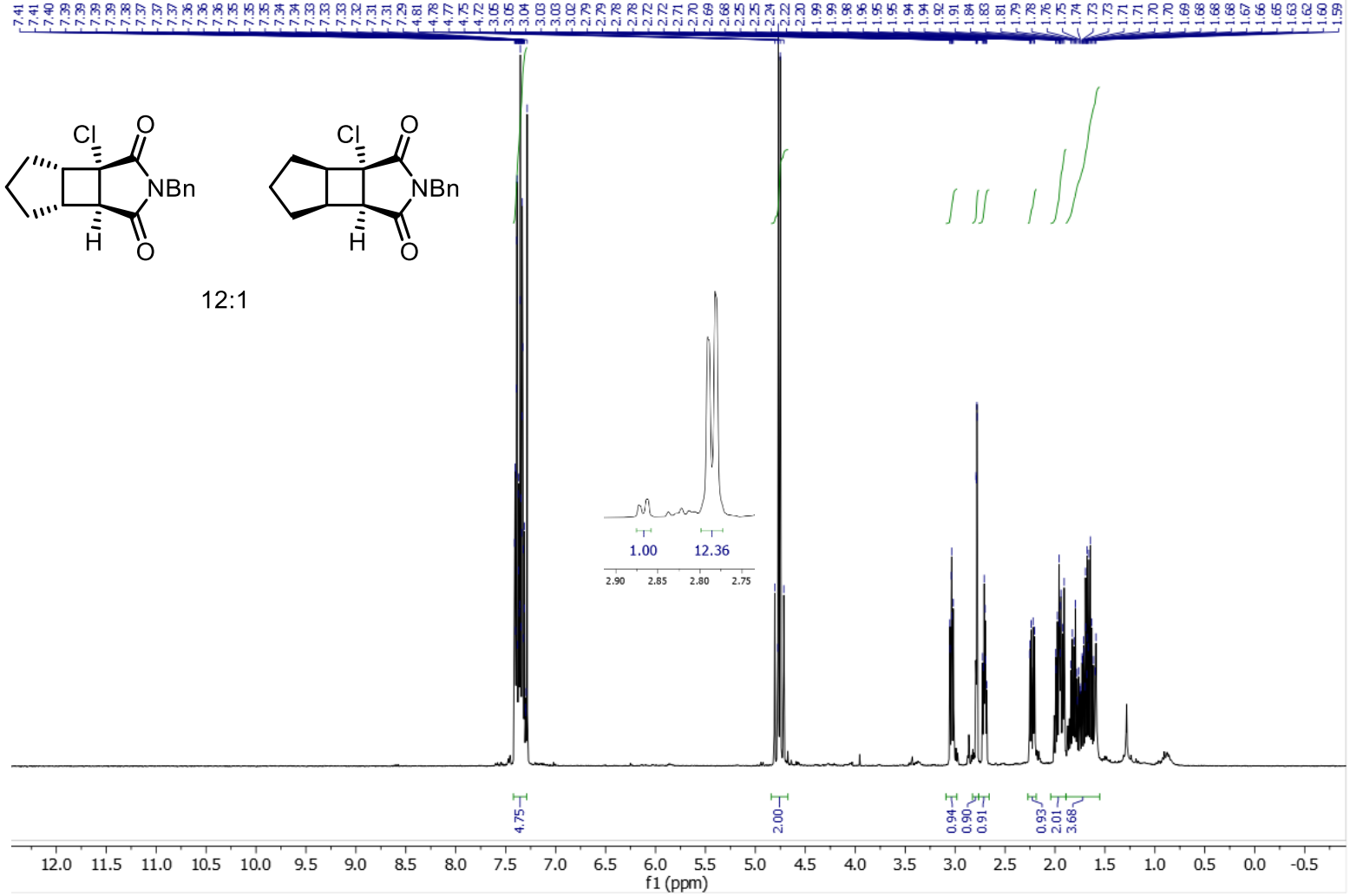

${ }^{13} \mathrm{C}$ NMR (100 MHz, $\mathrm{CDCl}_{3}$ ) of compound 12 (see procedure)

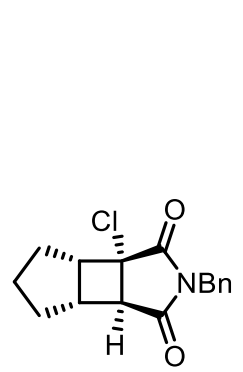

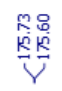

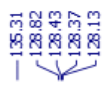

12:1
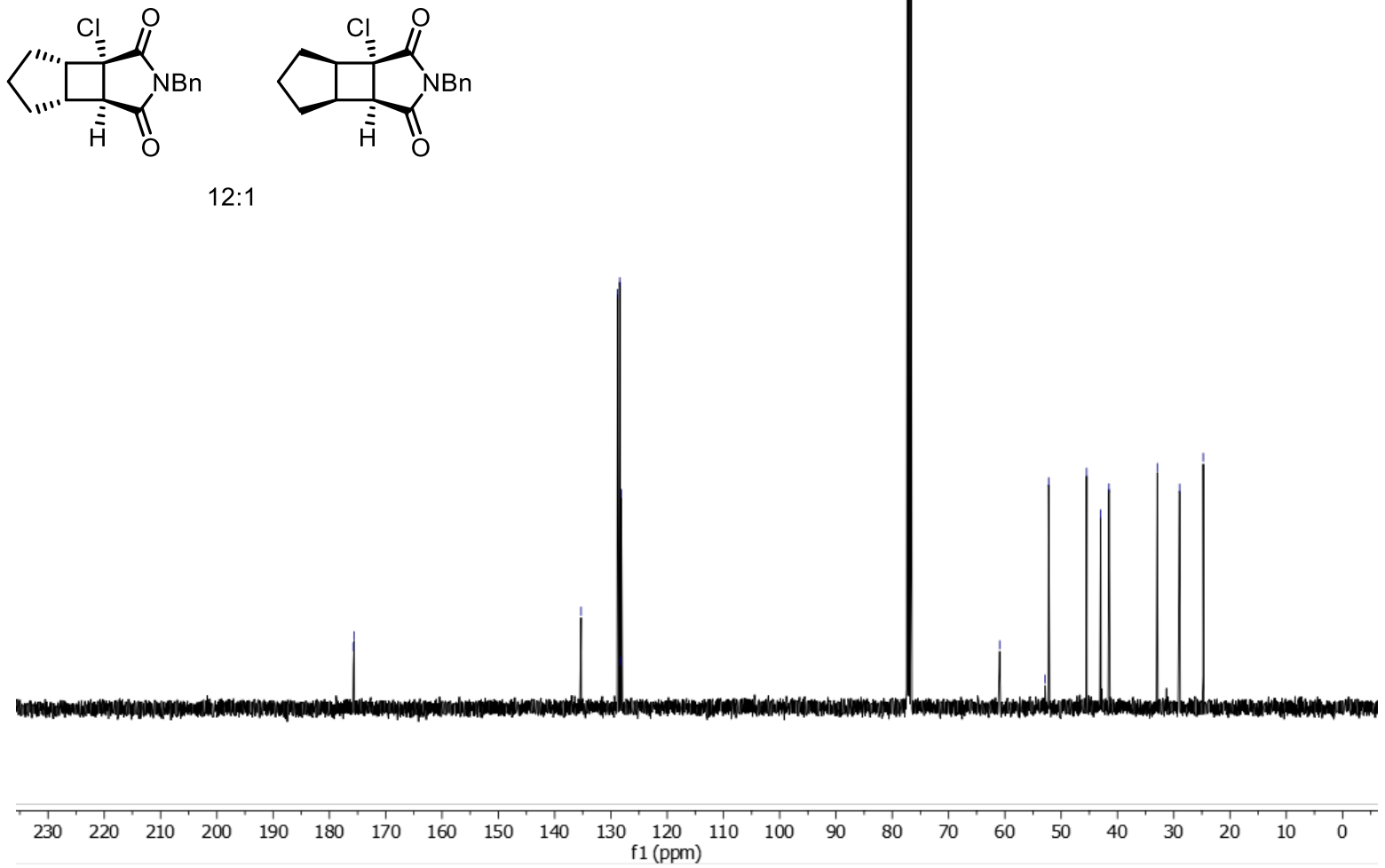
NOE NMR (500 MHz, $\mathrm{CDCl}_{3}$ ) of compound 12 (see procedure)

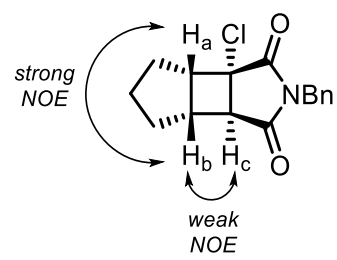

No $\mathrm{H}_{a}$ to $\mathrm{H}_{c}$ correlation
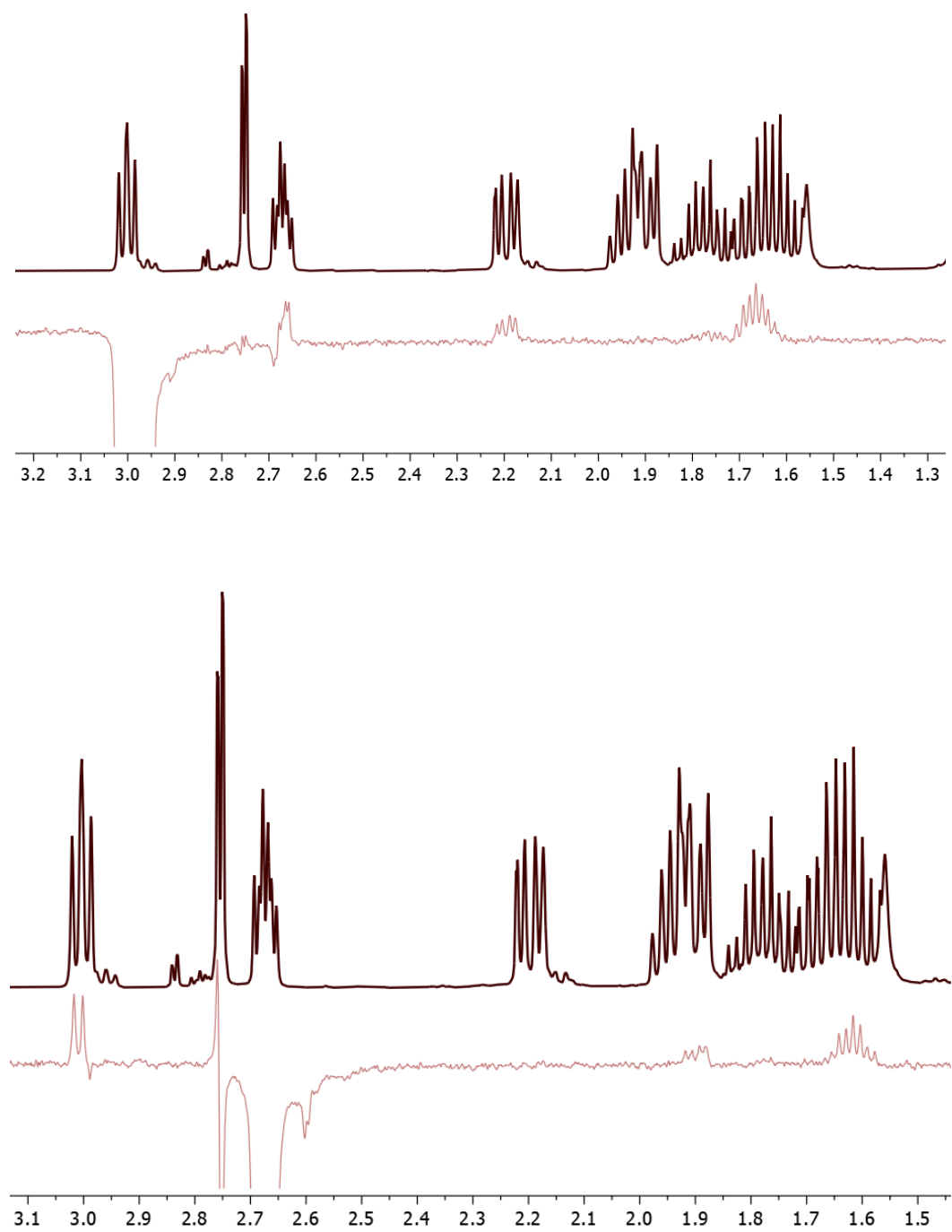
${ }^{1} \mathrm{H}$ NMR (400 MHz, $\mathrm{CDCl}_{3}$ ) of compound 13 (see procedure)

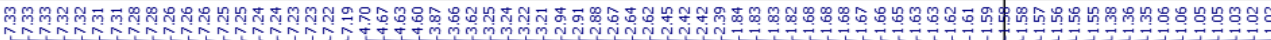
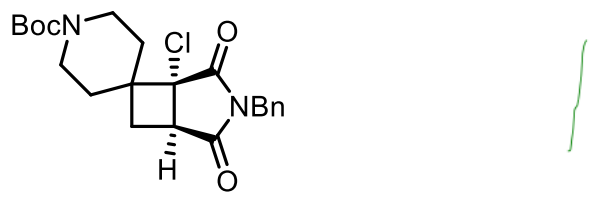

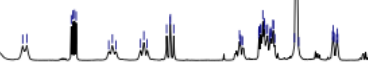

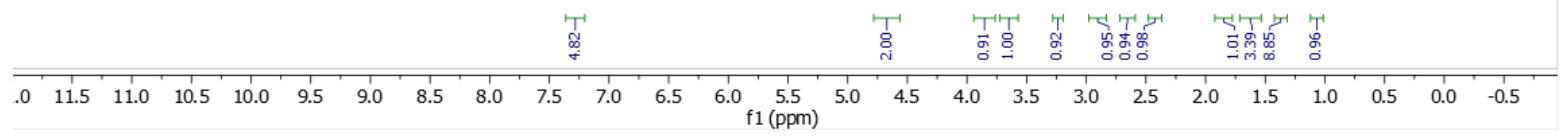

${ }^{13} \mathrm{C}$ NMR (100 MHz, $\left.\mathrm{CDCl}_{3}\right)$ of compound 13 (see procedure $)$

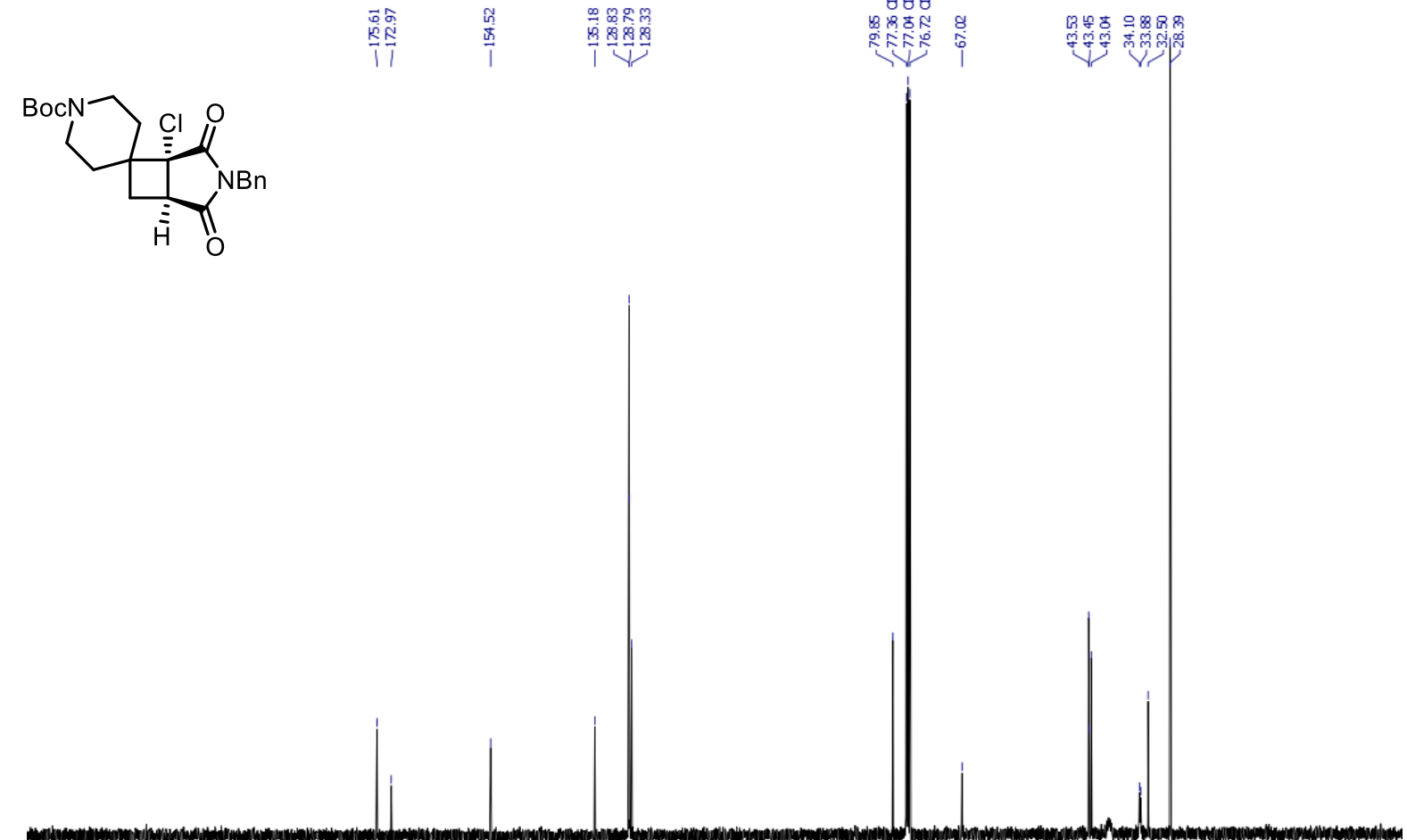

\begin{tabular}{|l|l|l|lllllllllllllllllllllllllllll}
\hline 40 & 230 & 220 & 210 & 200 & 190 & 180 & 170 & 160 & 150 & 140 & 130 & 120 & 110 & 100 & 90 & 80 & 70 & 60 & 50 & 40 & 30 & 20 & 10 & 0 & -10
\end{tabular} 
${ }^{1} \mathrm{H}$ NMR (400 MHz, $\mathrm{CDCl}_{3}$ ) of compound 14 (see procedure)<smiles>CN1C(=O)C2C(Cl)CC3CCOC3C2(Cl)C1=O</smiles>
要

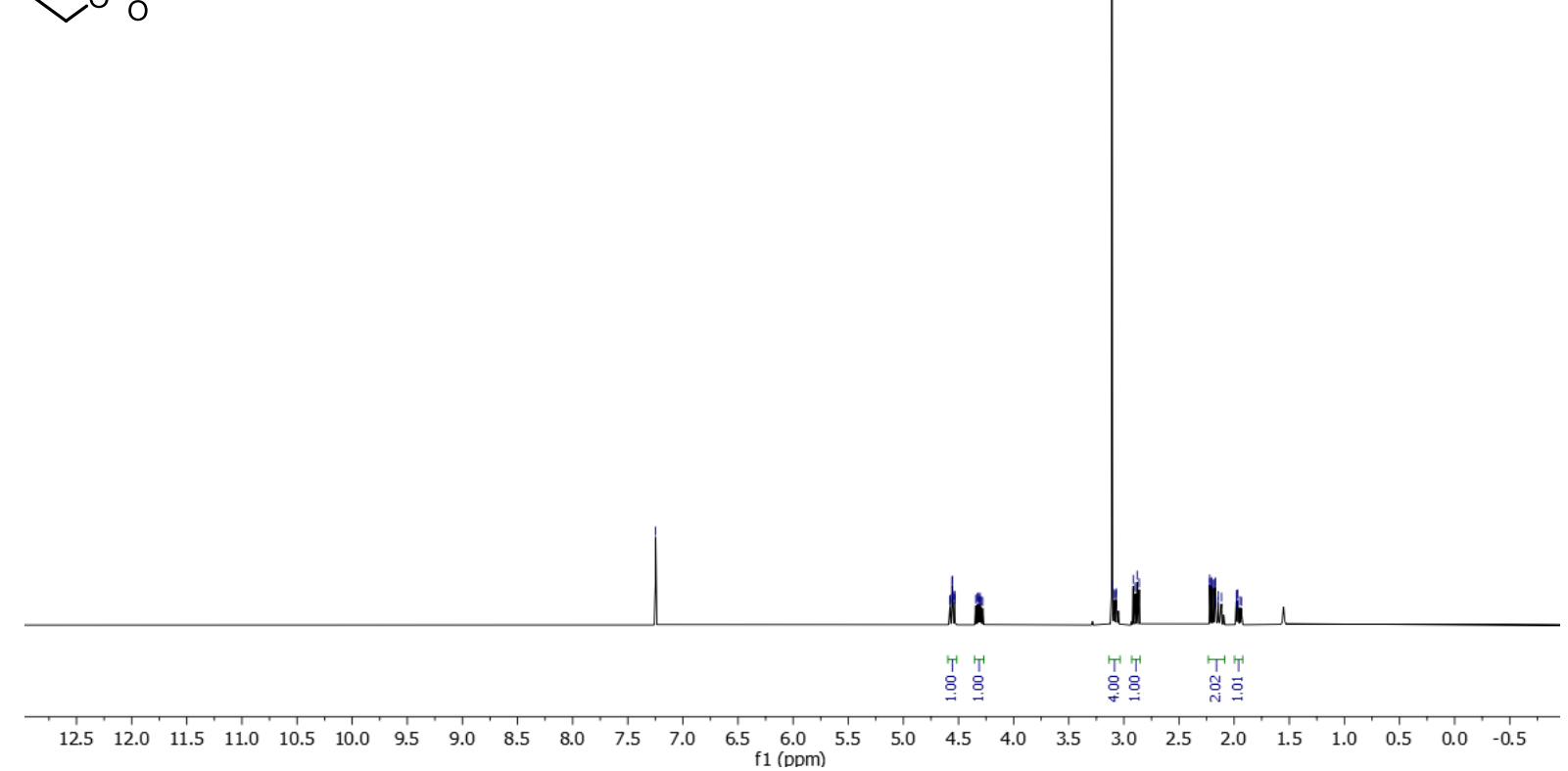

${ }^{13} \mathrm{C}$ NMR (100 MHz, $\mathrm{CDCl}_{3}$ ) of compound 14 (see procedure) ํํำ

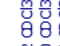<smiles>CN1C(=O)C2CC3(OCCCC3C2Cl)C1=O</smiles> 
${ }^{1} \mathrm{H}$ NMR (400 MHz, $\mathrm{CDCl}_{3}$ ) of compound 15 (see procedure)

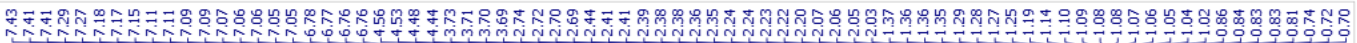
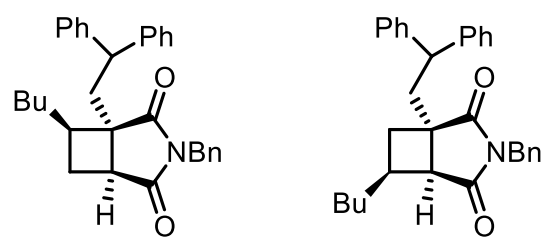

$18: 1$
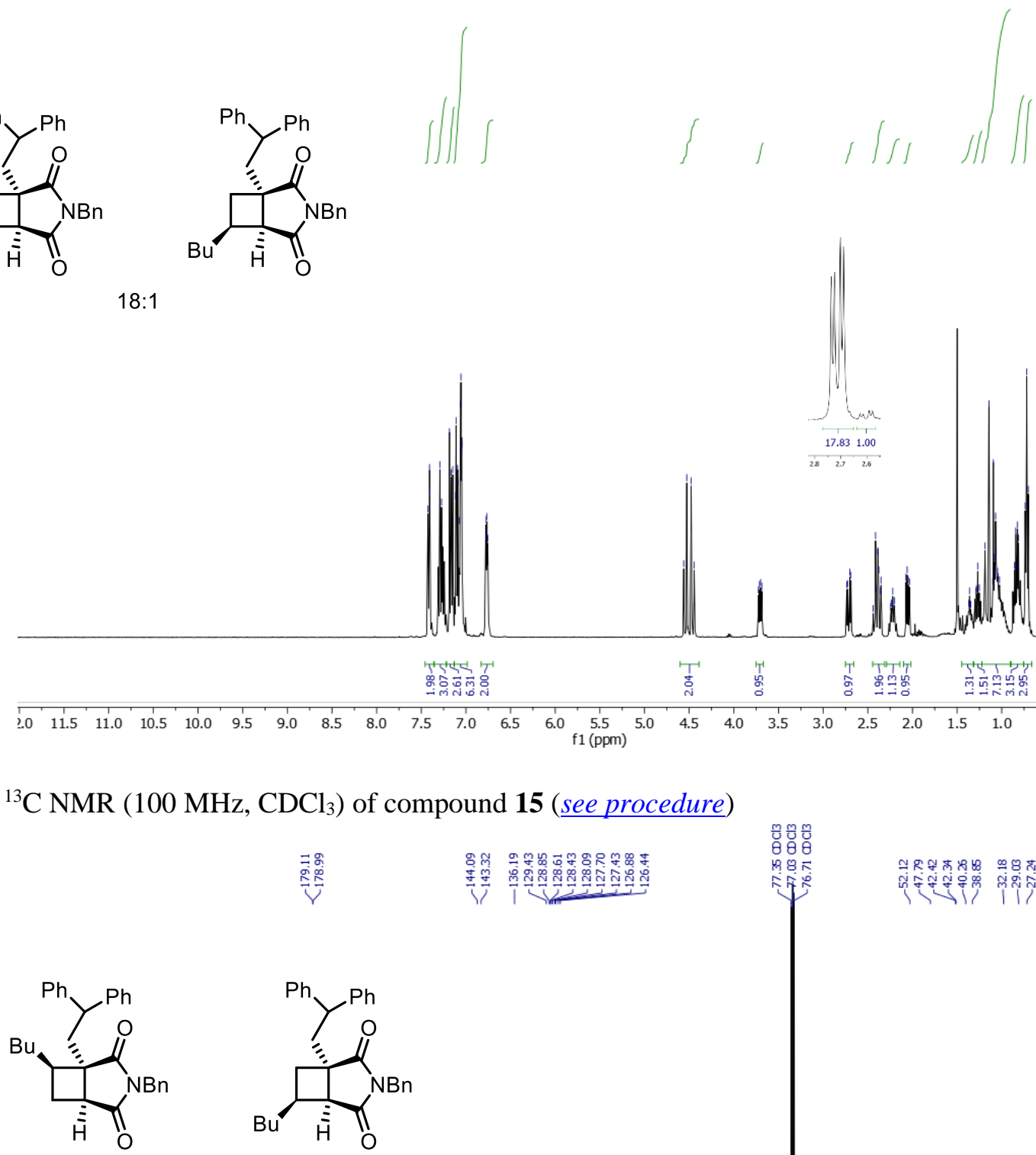

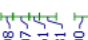

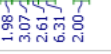

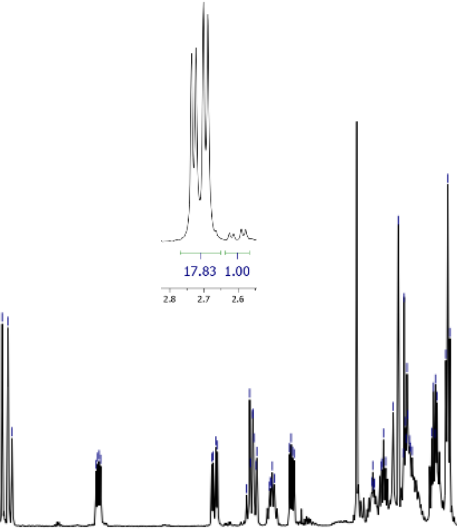

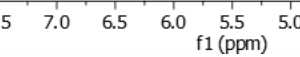

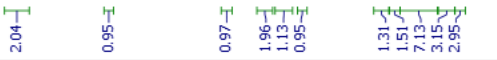

$\begin{array}{lllll}0 & 9.5 & 9.0 & 8.5 & 8.0\end{array}$

edure)

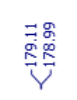

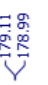

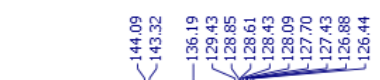

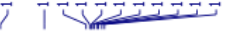

18:1

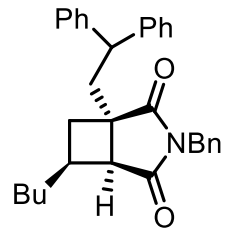

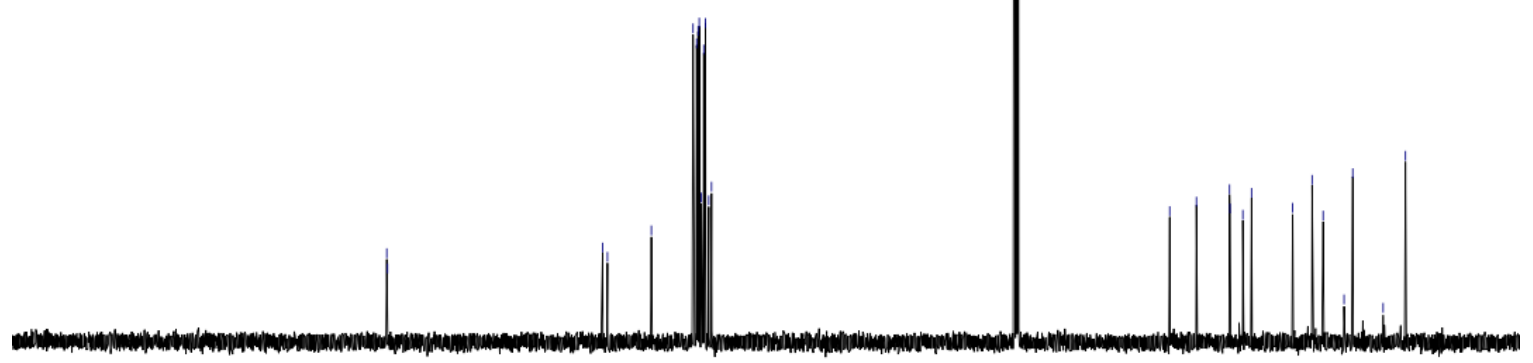

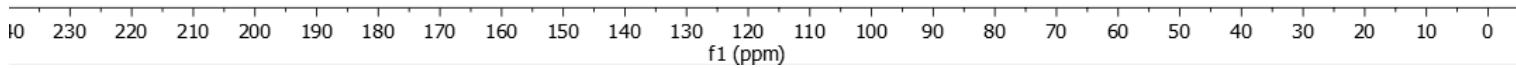


${ }^{1} \mathrm{H} \mathrm{NMR}\left(400 \mathrm{MHz}, \mathrm{CDCl}_{3}\right.$ ) of compound 16 (see procedure)

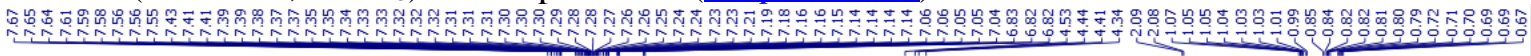
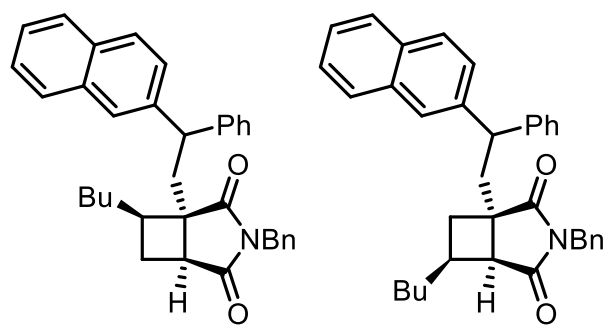

$9: 1$ r.r. $56: 44$ d.r.

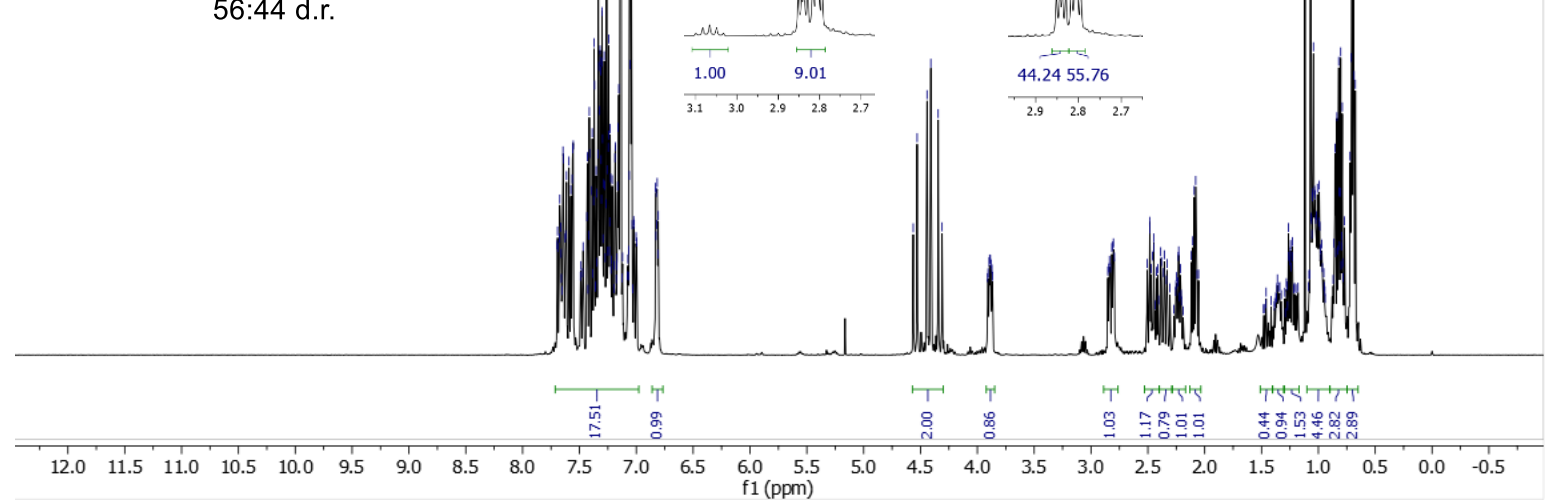

${ }^{13} \mathrm{C} \mathrm{NMR}\left(100 \mathrm{MHz}, \mathrm{CDCl}_{3}\right)$ of compound 16 (see procedure)

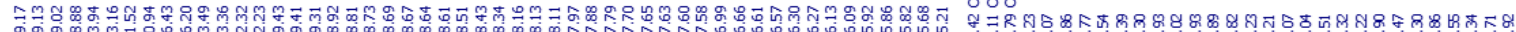

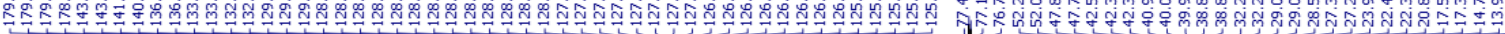

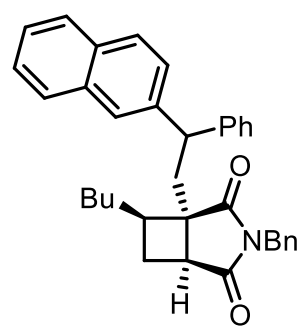

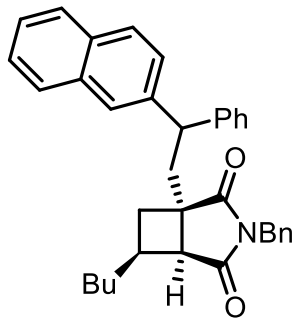

$9: 1$ r.r. $56: 44$ d.r.
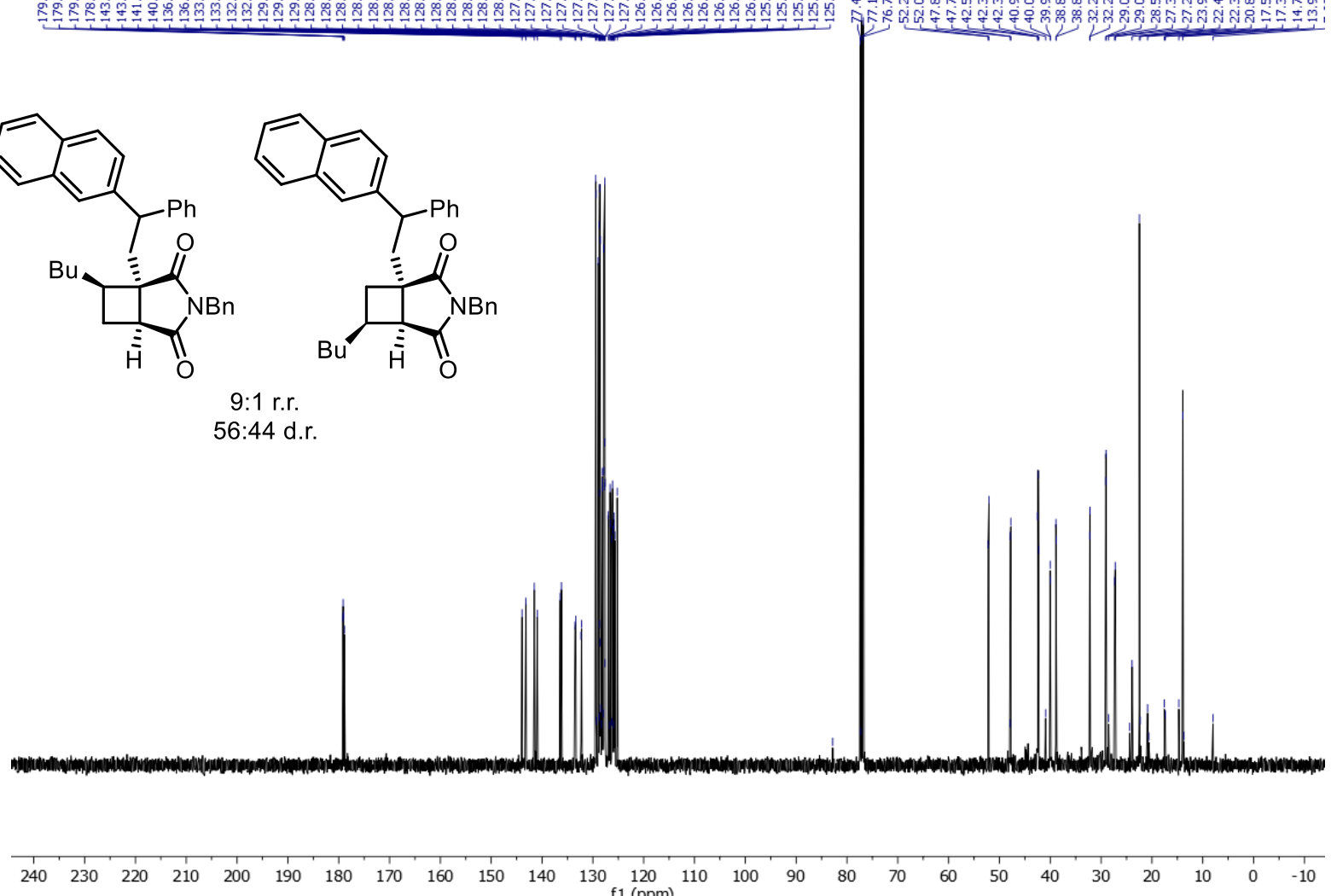
${ }^{1} \mathrm{H} \mathrm{NMR}\left(400 \mathrm{MHz}, \mathrm{CDCl}_{3}\right.$ ) of compound 17 (see procedure)

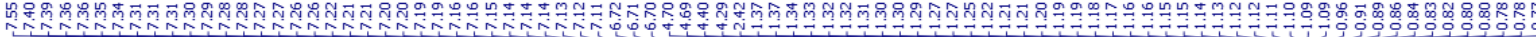

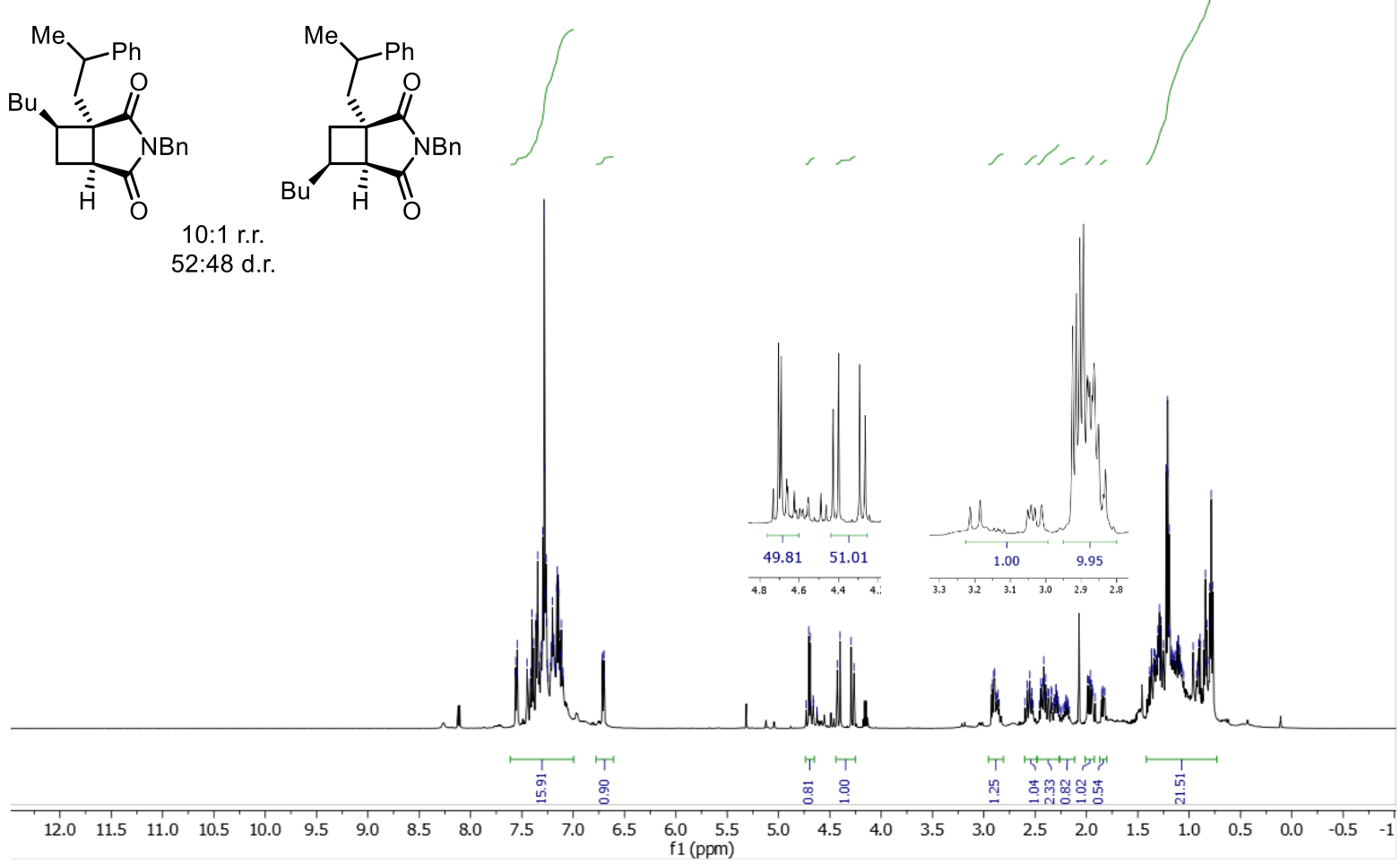

${ }^{13} \mathrm{C}$ NMR (100 MHz, $\mathrm{CDCl}_{3}$ ) of compound 17 (see procedure)

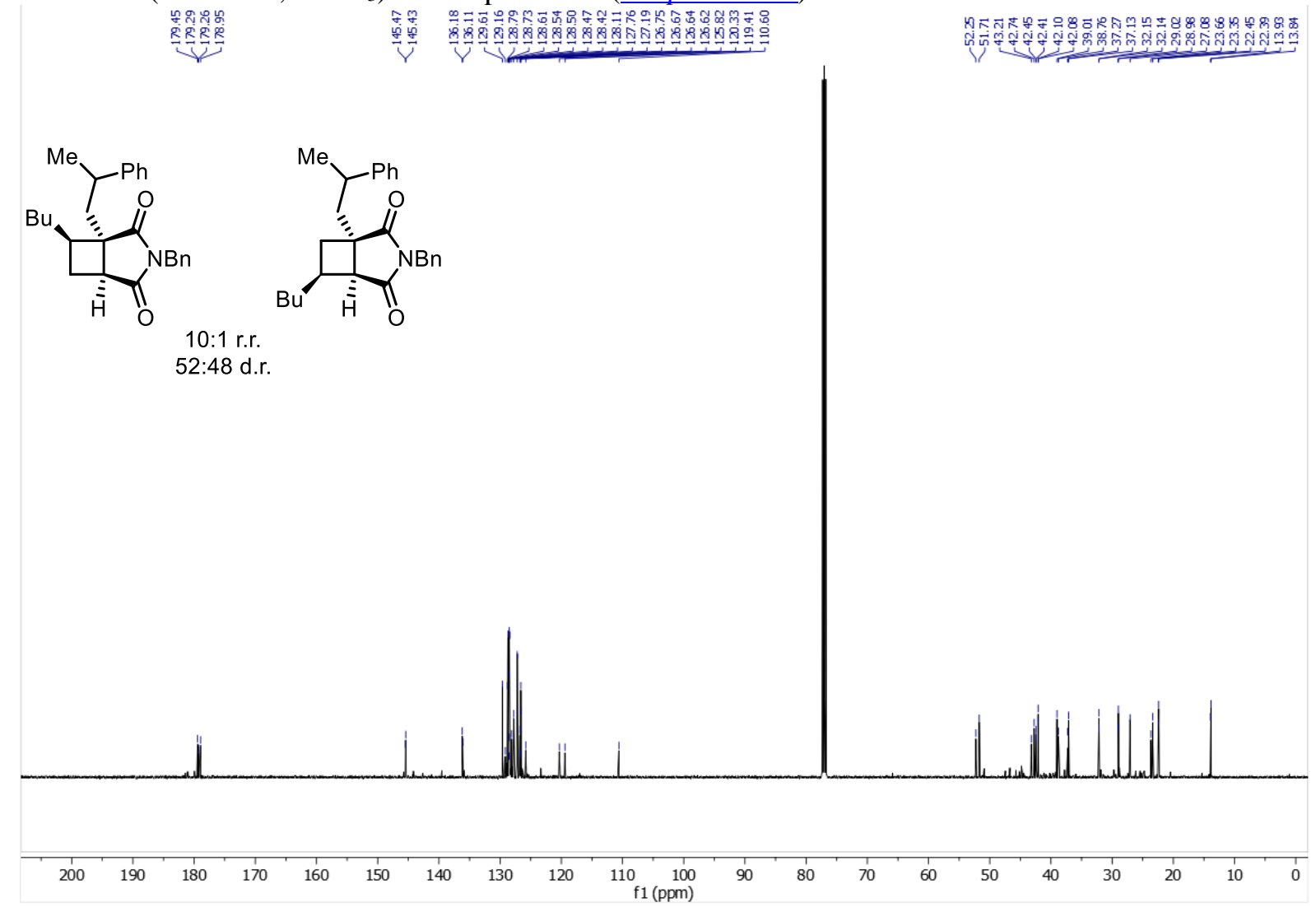


${ }^{1} \mathrm{H} \mathrm{NMR}\left(400 \mathrm{MHz}, \mathrm{CDCl}_{3}\right.$ ) of compound 18 (see procedure)

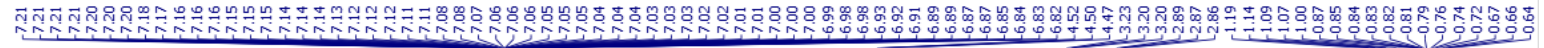
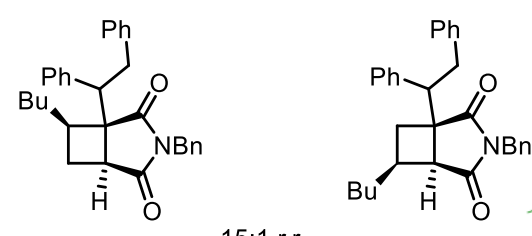

$15: 1$ r.r.

$54: 46$ d.r.
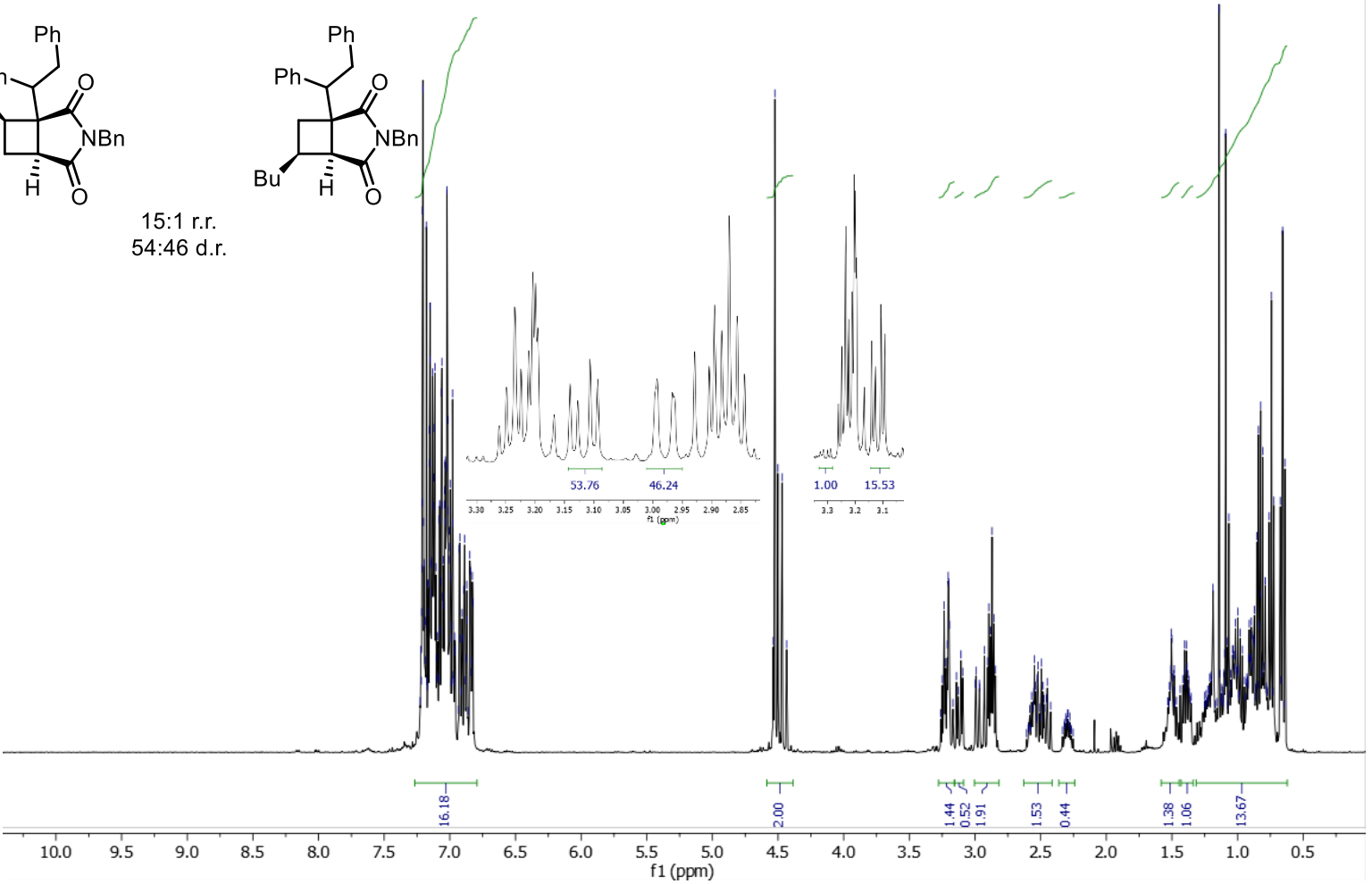

${ }^{13} \mathrm{C} \mathrm{NMR}\left(100 \mathrm{MHz}, \mathrm{CDCl}_{3}\right)$ of compound 18 (see procedure)

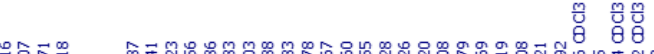

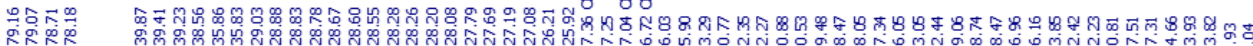

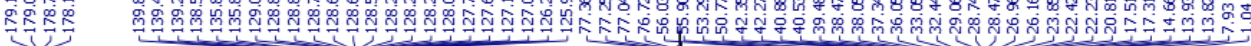<smiles>O=C1[NH2+]C(=O)C(C(Br)Cc2ccccc2)C1Cc1ccccc1</smiles>

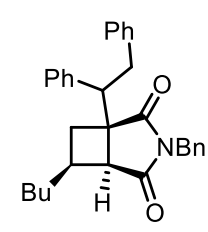

$15: 1$ r.r. $54: 46$ d.r.

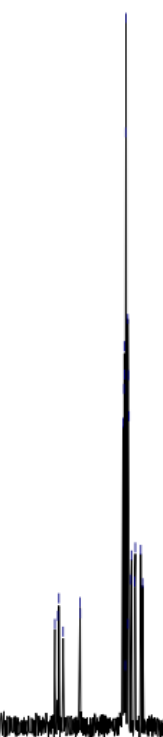

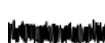

$\begin{array}{lllllllllllllll}30 & 220 & 210 & 200 & 190 & 180 & 170 & 160 & 150 & 140 & 130 & 120 & 110 & 100 & 90 \\ \mathrm{f} 1(\mathrm{ppm}) & & & & \end{array}$ $\begin{array}{lllll}80 & 70 & 60 & 50\end{array}$ $30 \quad 20$ $-10$ 
${ }^{1} \mathrm{H}$ NMR (400 MHz, $\mathrm{CDCl}_{3}$ ) of compound 19 (see procedure)

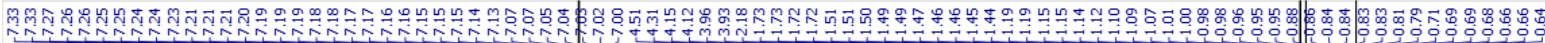

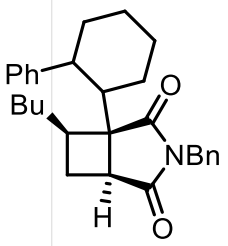

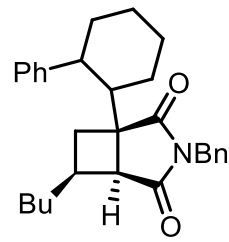

$9: 1$ r.r. $61: 39$ d.r.

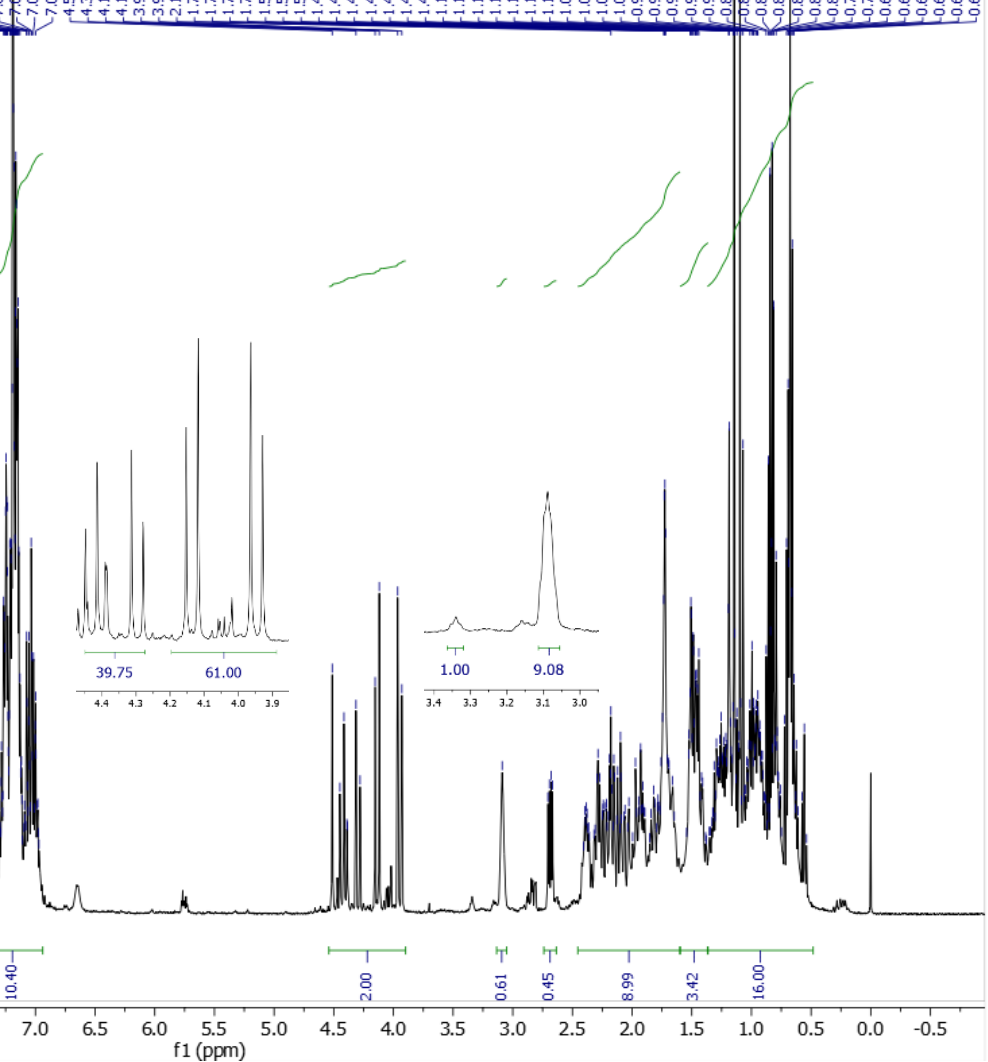

${ }^{13} \mathrm{C}$ NMR $\left(100 \mathrm{MHz}, \mathrm{CDCl}_{3}\right.$ ) of compound $\mathbf{1 9}$ (see procedure)

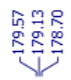

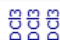

舟

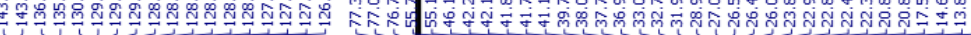<smiles>O=C1C2C(Br)C3CCCC(Br)C3C[C@@H]2C(=O)N1Br</smiles>

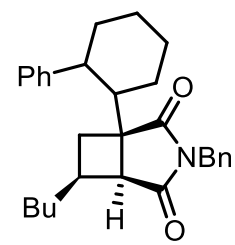

9:1 r.r.

61:39 d.r.

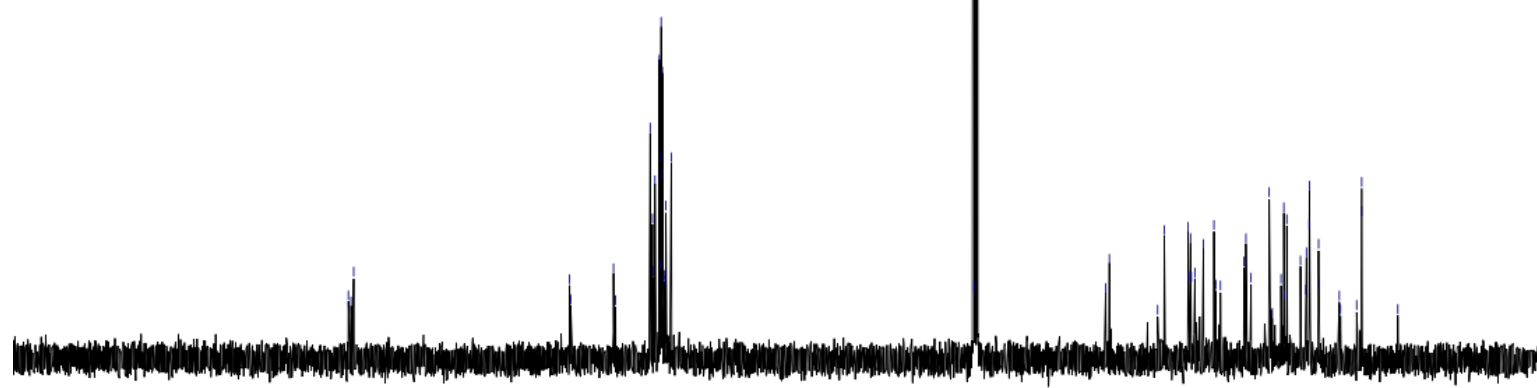

$\begin{array}{lllllllllllllllllllllllllll}230 & 220 & 210 & 200 & 190 & 180 & 170 & 160 & 150 & 140 & 130 & 120 & 110 & 100 & 90 & 80 & 70 & 60 & 50 & 40 & 30 & 20 & 10 & 0 & -10\end{array}$ 
${ }^{1} \mathrm{H}$ NMR (500 MHz, $\mathrm{CDCl}_{3}$ ) of compound 20 (see procedure)

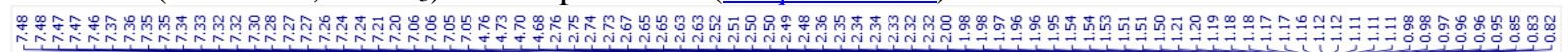<smiles>O=C1[C@H]2CC(CCc3ccccc3)[C@@]2(CCc2ccccc2)C(=O)N1Cc1ccccc1</smiles>

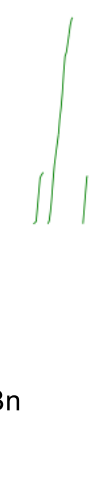

$10: 1$ rr.<smiles>CC(C)(C)C1C[C@@]2(CCc3ccccc3)C(=O)C(=O)[C@@H]3[C@@H]1CC32Cc1ccccc1</smiles>

III , I |11

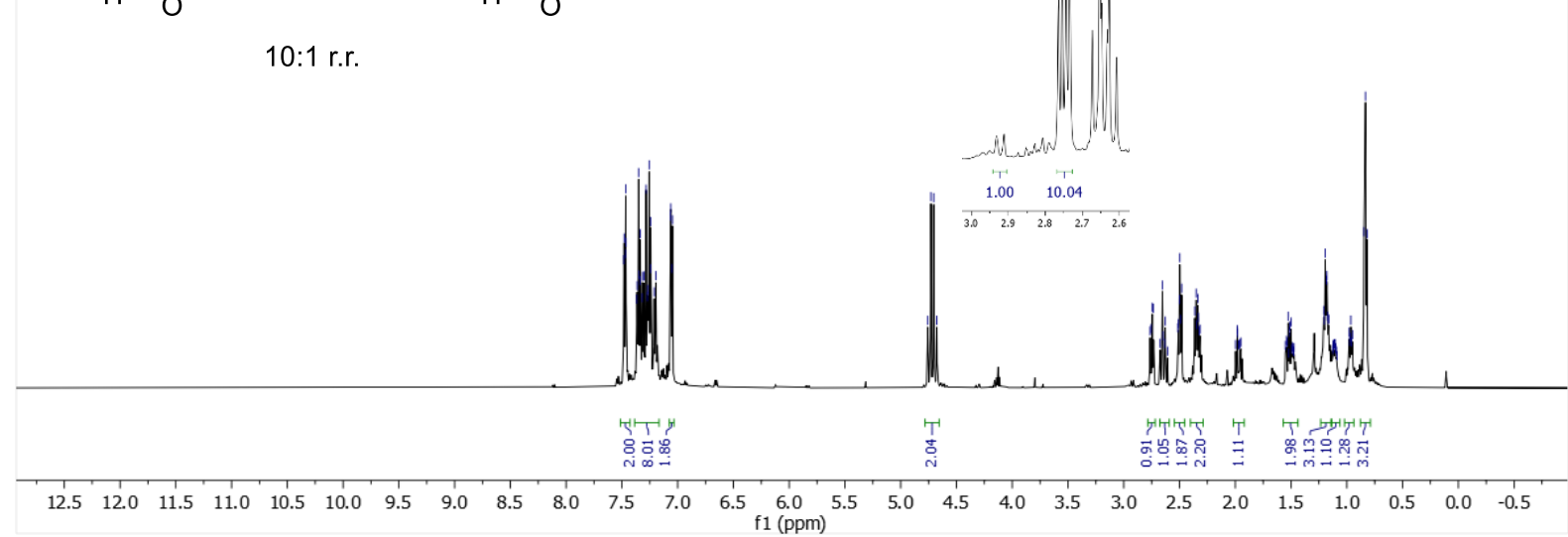

${ }^{13} \mathrm{C}$ NMR (125 MHz, $\mathrm{CDCl}_{3}$ ) of compound 20 (see procedure)

量篦

1

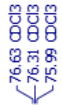

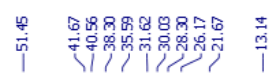<smiles>CC(C)(C)[C@H]1C[C@H]2C(=O)N(Cc3ccccc3)C(=O)[C@@]21CCc1ccccc1</smiles><smiles>Cc1ccccc1</smiles><smiles>CC[C@]12C[C@@H](Br)[C@H]1C(=O)NC2=O</smiles>

10:1 rr.
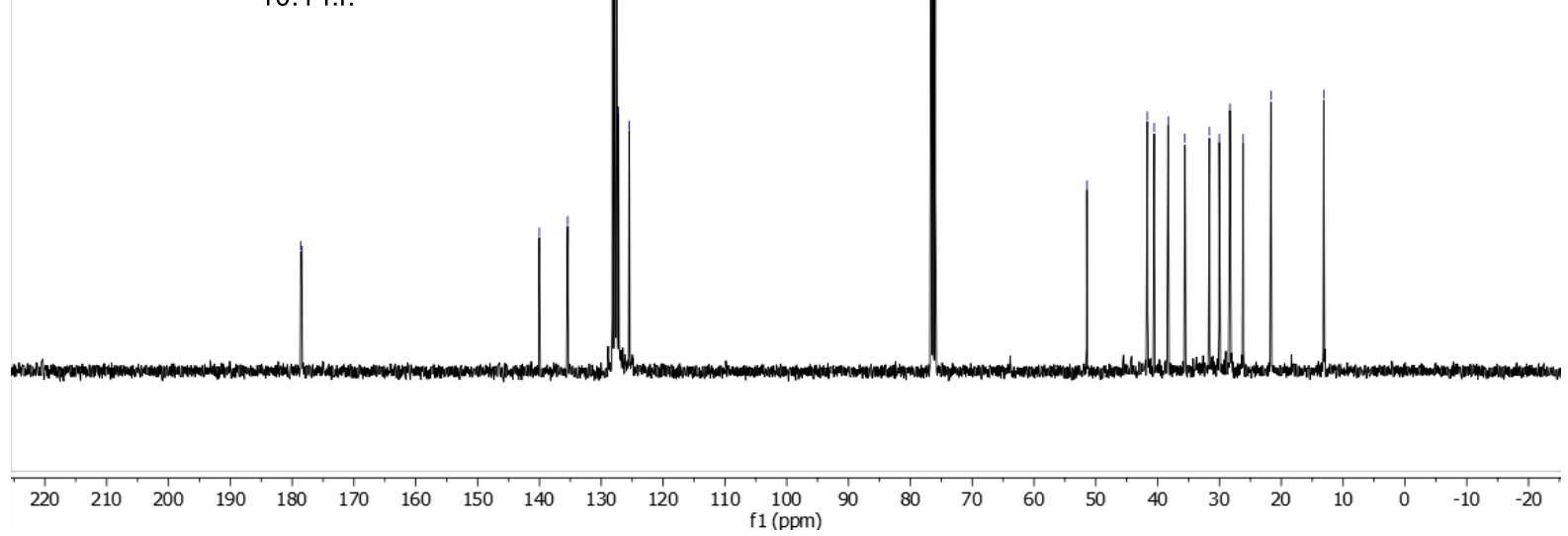

S82 
${ }^{1} \mathrm{H} \mathrm{NMR}\left(400 \mathrm{MHz}, \mathrm{CDCl}_{3}\right.$ ) of compound 21 (see procedure)

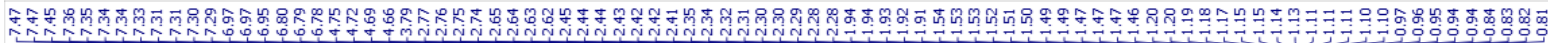<smiles></smiles><smiles>COc1ccc(CCC23CC(Br)[C@H]2C(=O)[NH2+]C3=O)cc1</smiles>

12:1 r.r.

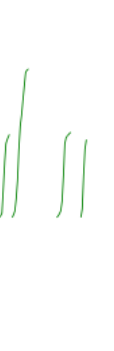

$\|$
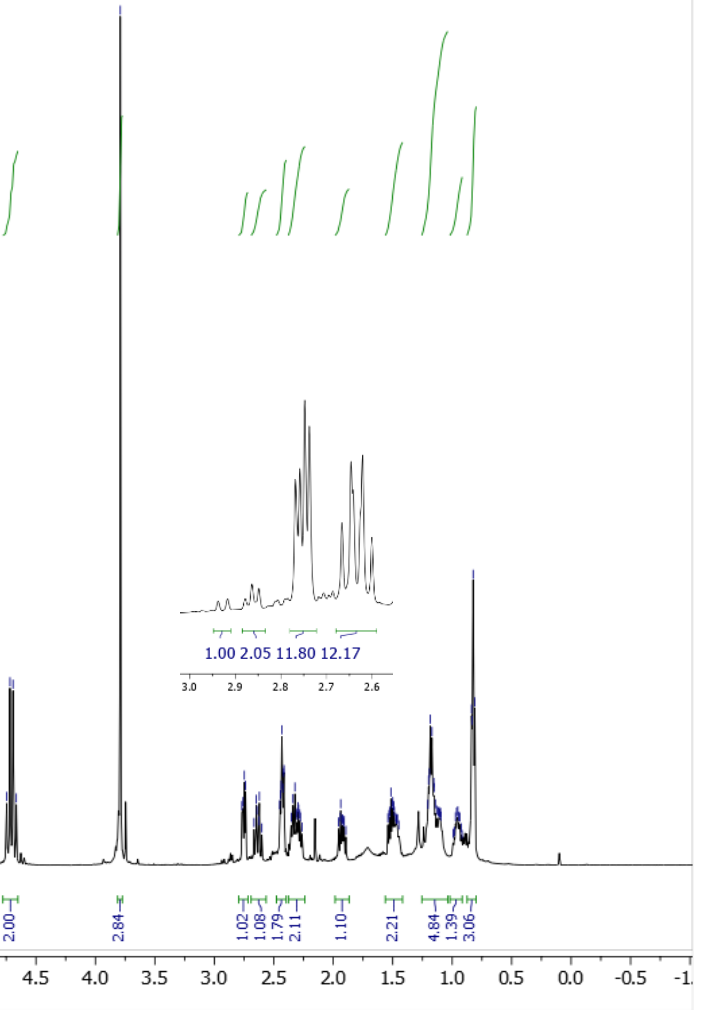

${ }^{13} \mathrm{C}$ NMR (100 MHz, $\mathrm{CDCl}_{3}$ ) of compound 21 (see procedure)

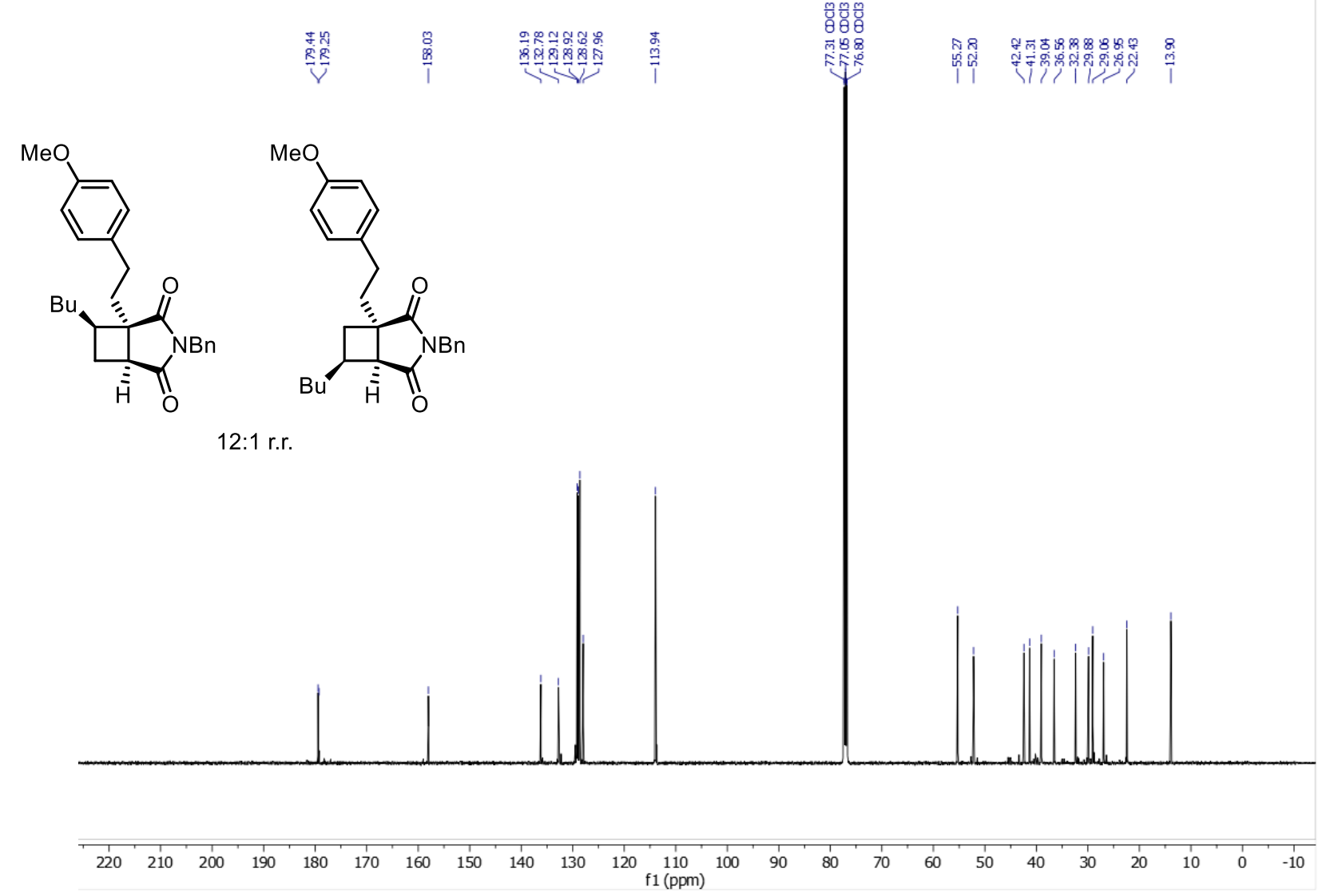


${ }^{1} \mathrm{H}$ NMR (400 MHz, $\mathrm{CDCl}_{3}$ ) of compound 22 (see procedure)

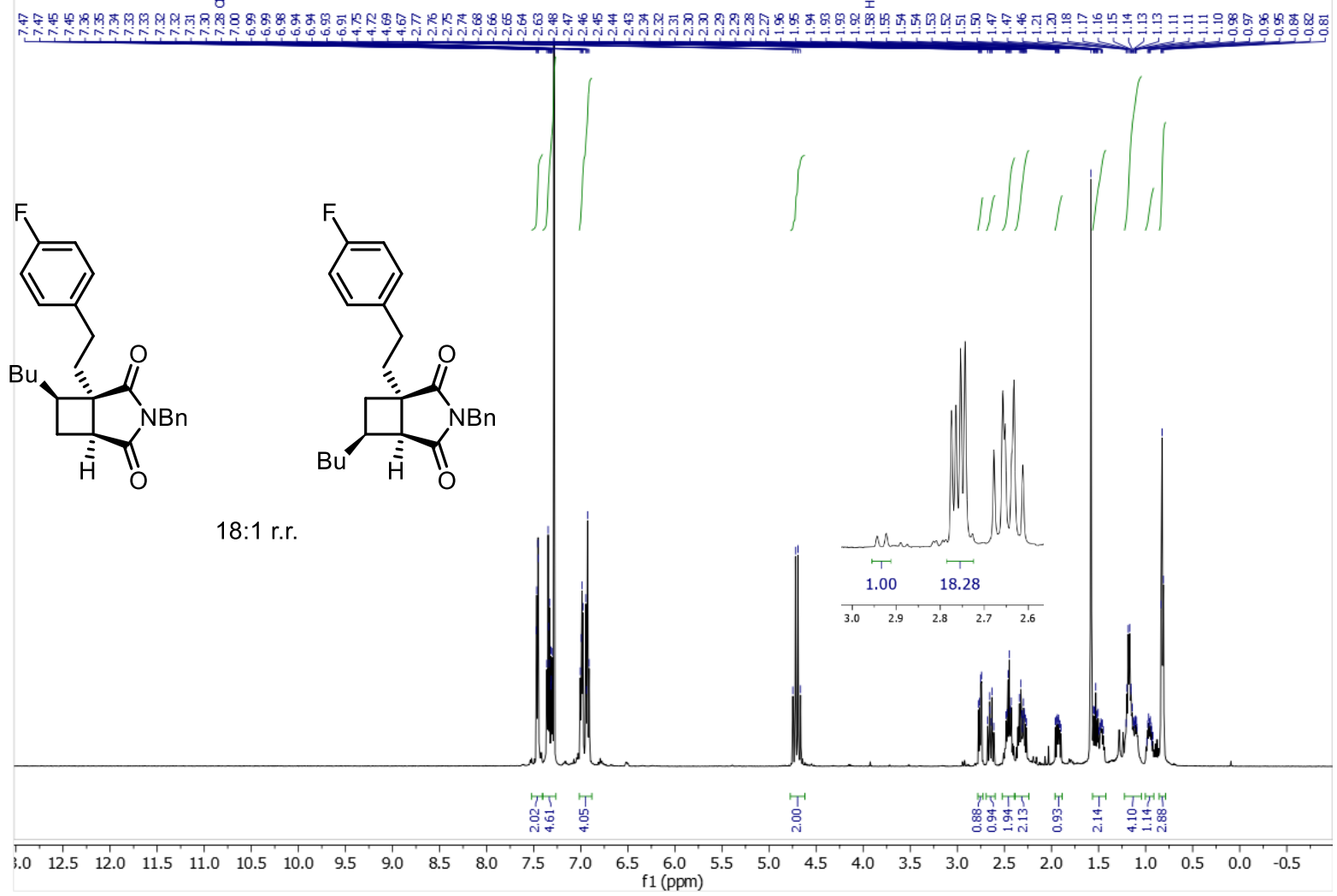

${ }^{13} \mathrm{C}$ NMR (100 MHz, $\mathrm{CDCl}_{3}$ ) of compound 22 (see procedure)
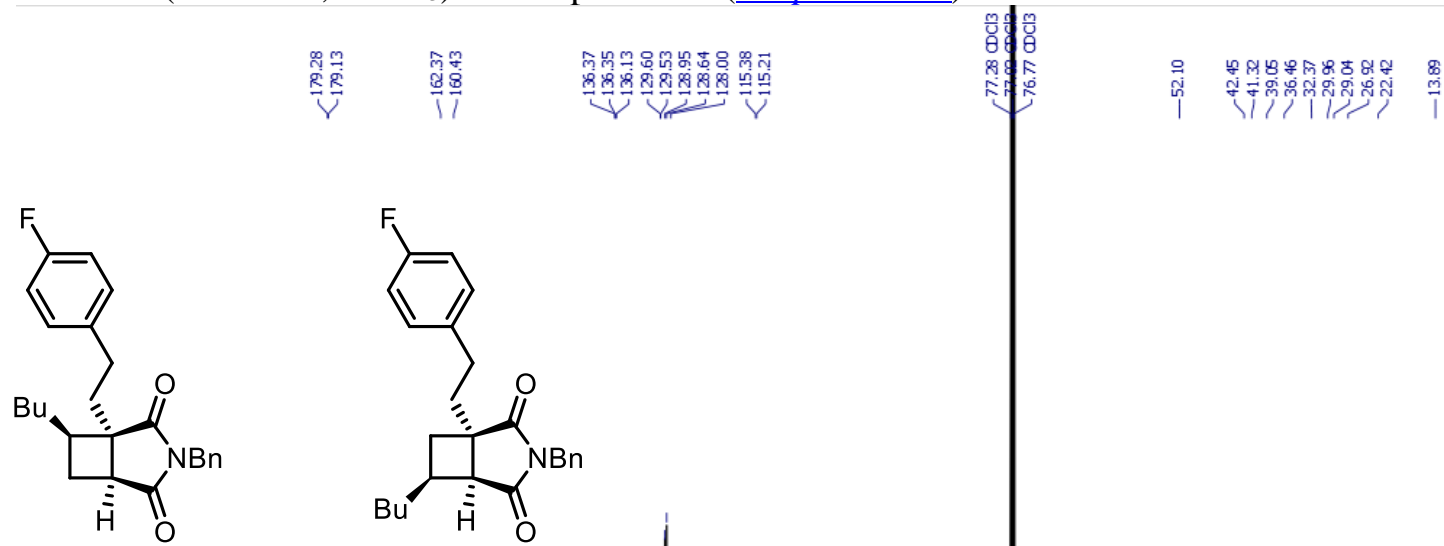

$18: 1$ r.r.

$\begin{array}{lllllllllll}220 & 210 & 200 & 190 & 180 & 170 & 160 & 150 & 140 & 130 & 120 \underset{\mathrm{f} 1(\mathrm{ppm})}{110}\end{array}$ 
${ }^{1} \mathrm{H}$ NMR (400 MHz, $\mathrm{CDCl}_{3}$ ) of compound 23 (see procedure) 觉

웅

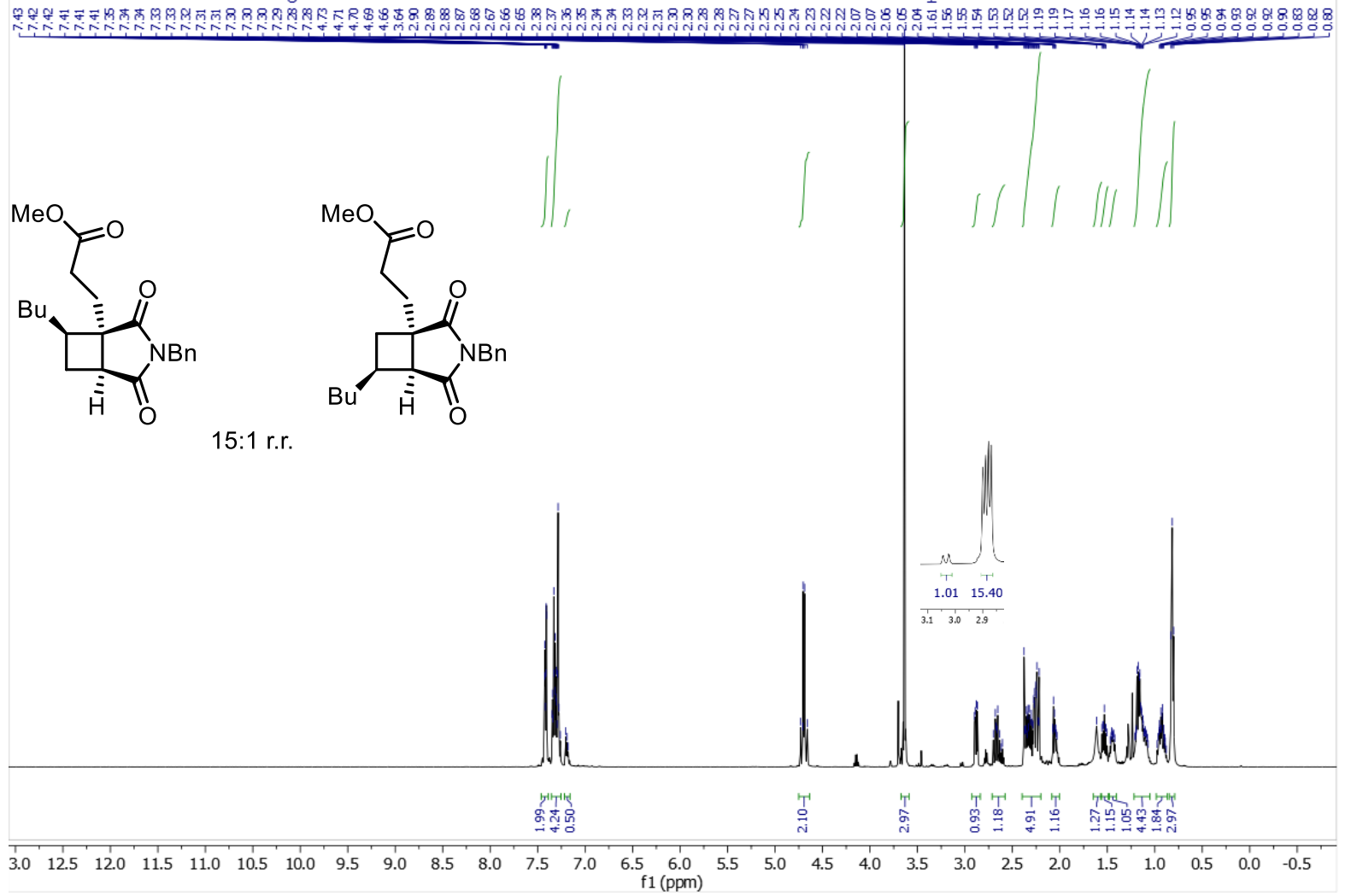

${ }^{13} \mathrm{C}$ NMR (100 MHz, $\left.\mathrm{CDCl}_{3}\right)$ of compound 23 (see procedure)

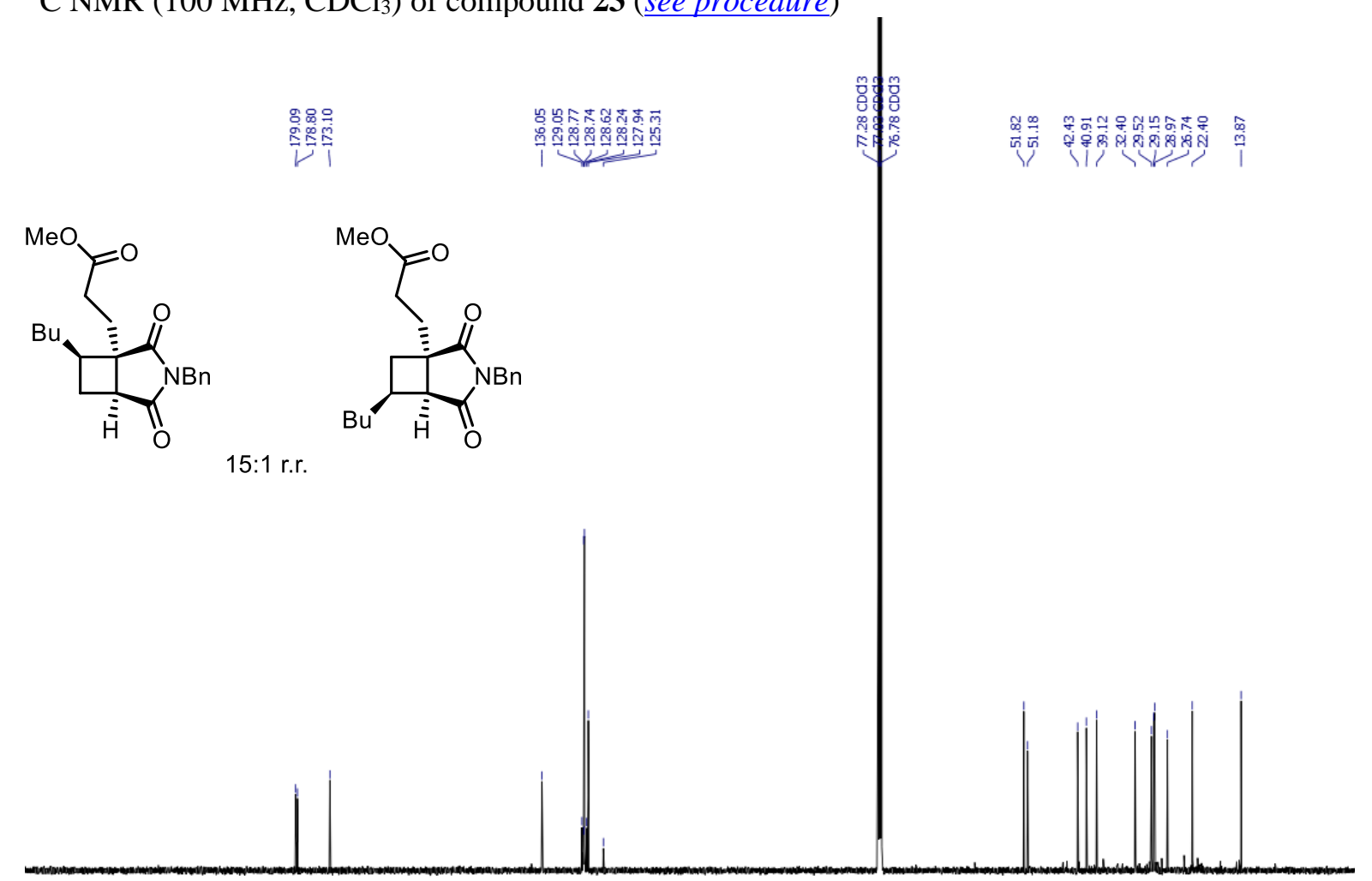

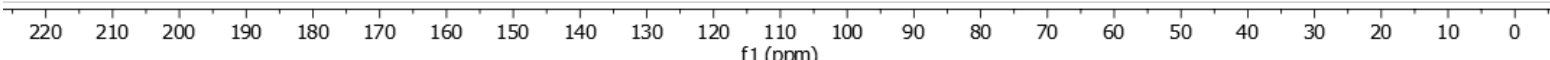


${ }^{1} \mathrm{H}$ NMR (400 MHz, $\mathrm{CDCl}_{3}$ ) of compound 24 (see procedure)

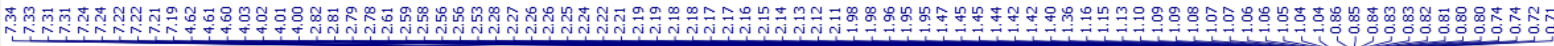
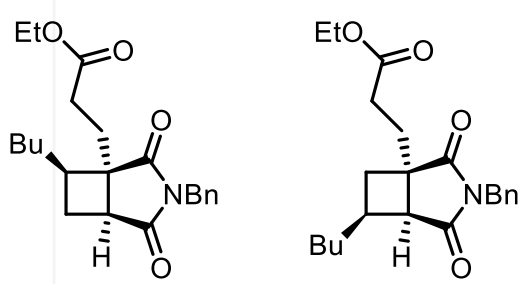

$11: 1$

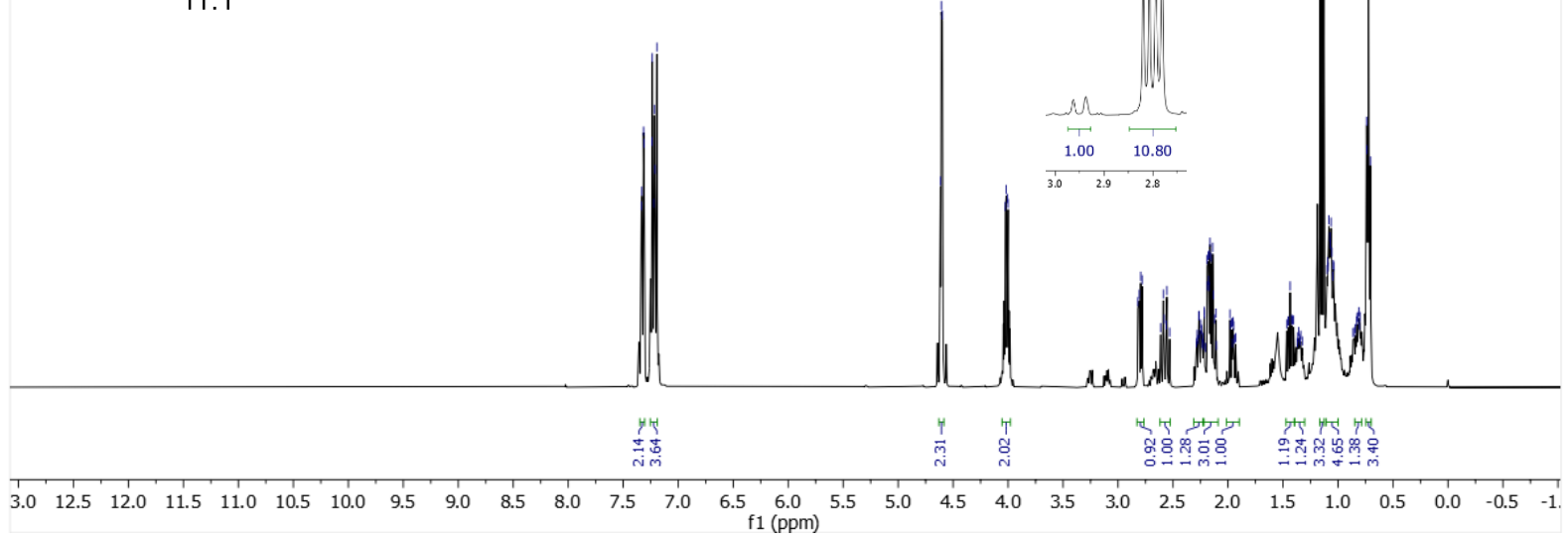

${ }^{13} \mathrm{C} \mathrm{NMR}\left(100 \mathrm{MHz}, \mathrm{CDCl}_{3}\right.$ ) of compound 24 (see procedure)

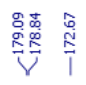
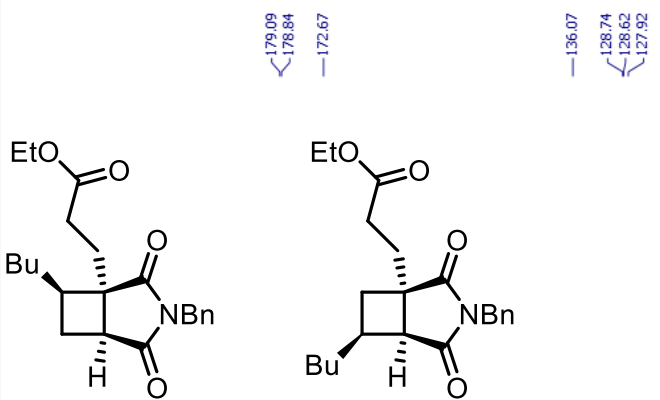

$11: 1$

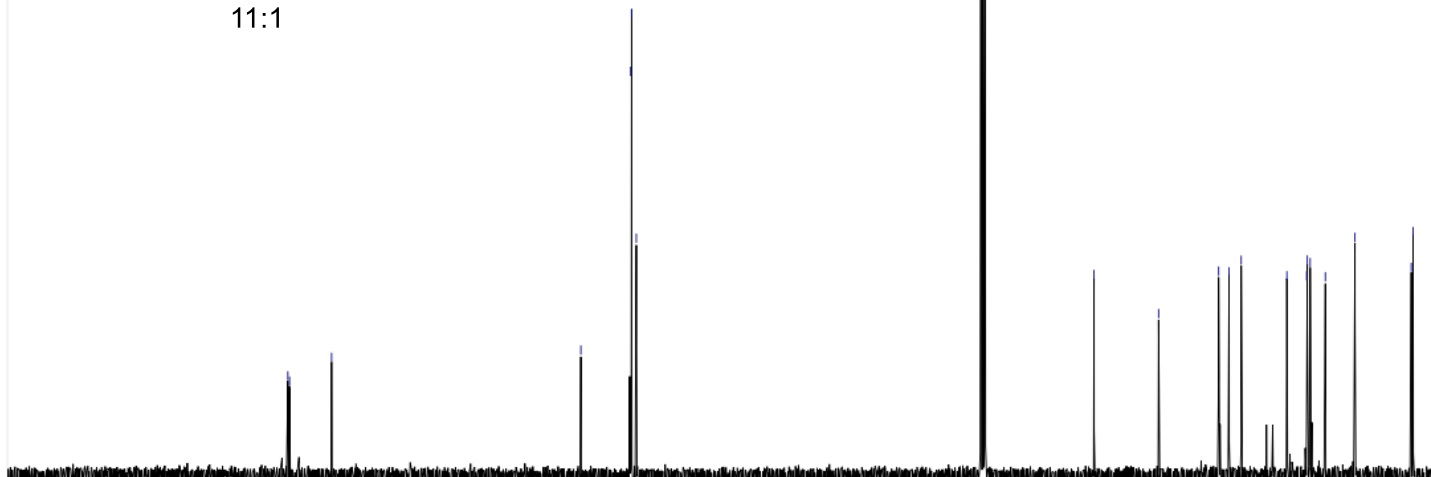

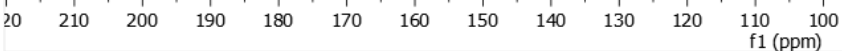


${ }^{1} \mathrm{H} \mathrm{NMR}\left(400 \mathrm{MHz}, \mathrm{CDCl}_{3}\right.$ ) of compound 25 (see procedure)

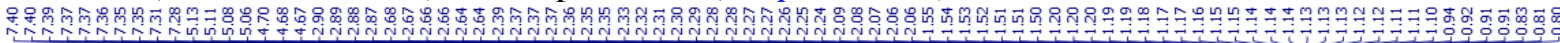

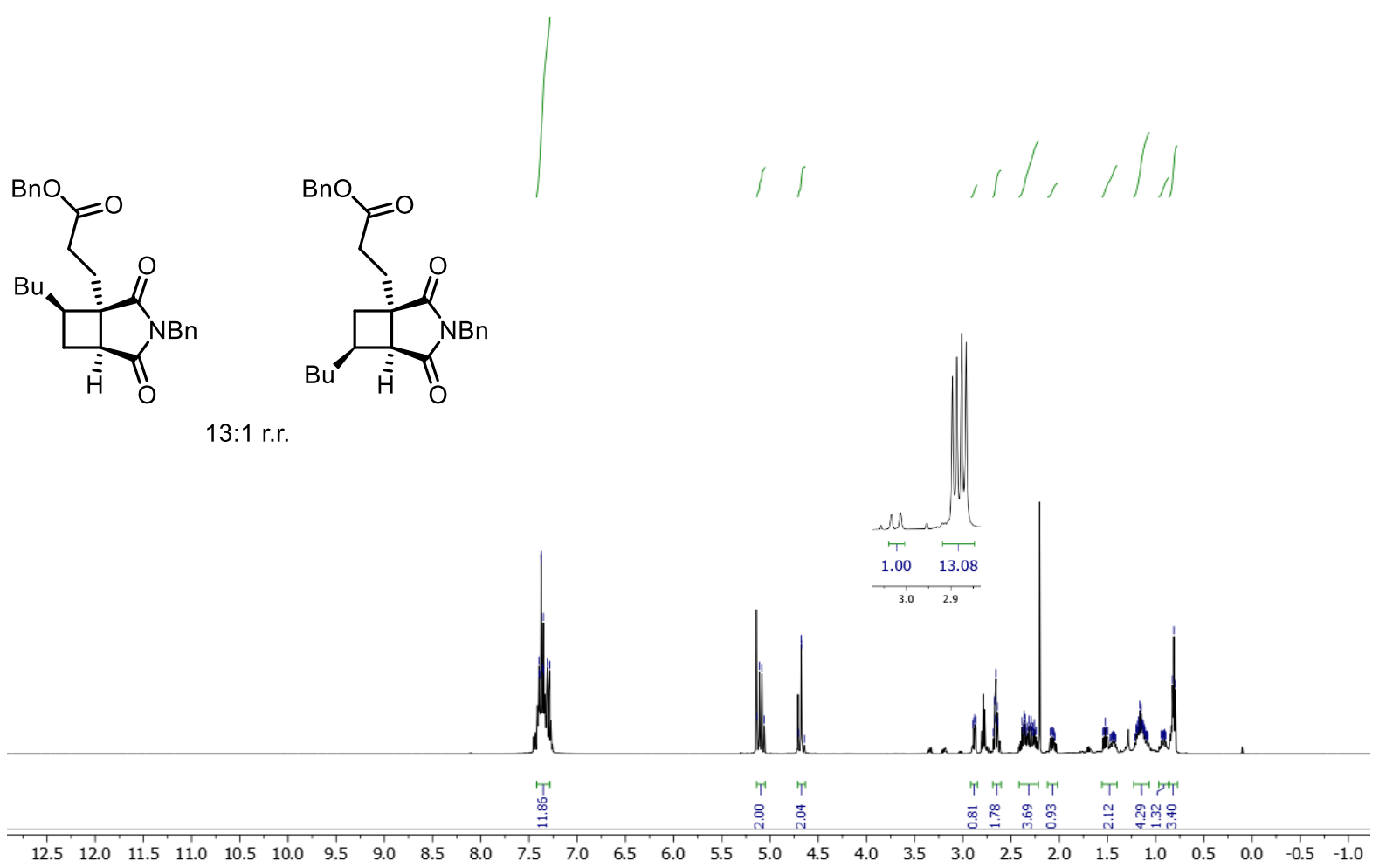

${ }^{13} \mathrm{C}$ NMR (100 MHz, $\mathrm{CDCl}_{3}$ ) of compound 25 (see procedure)

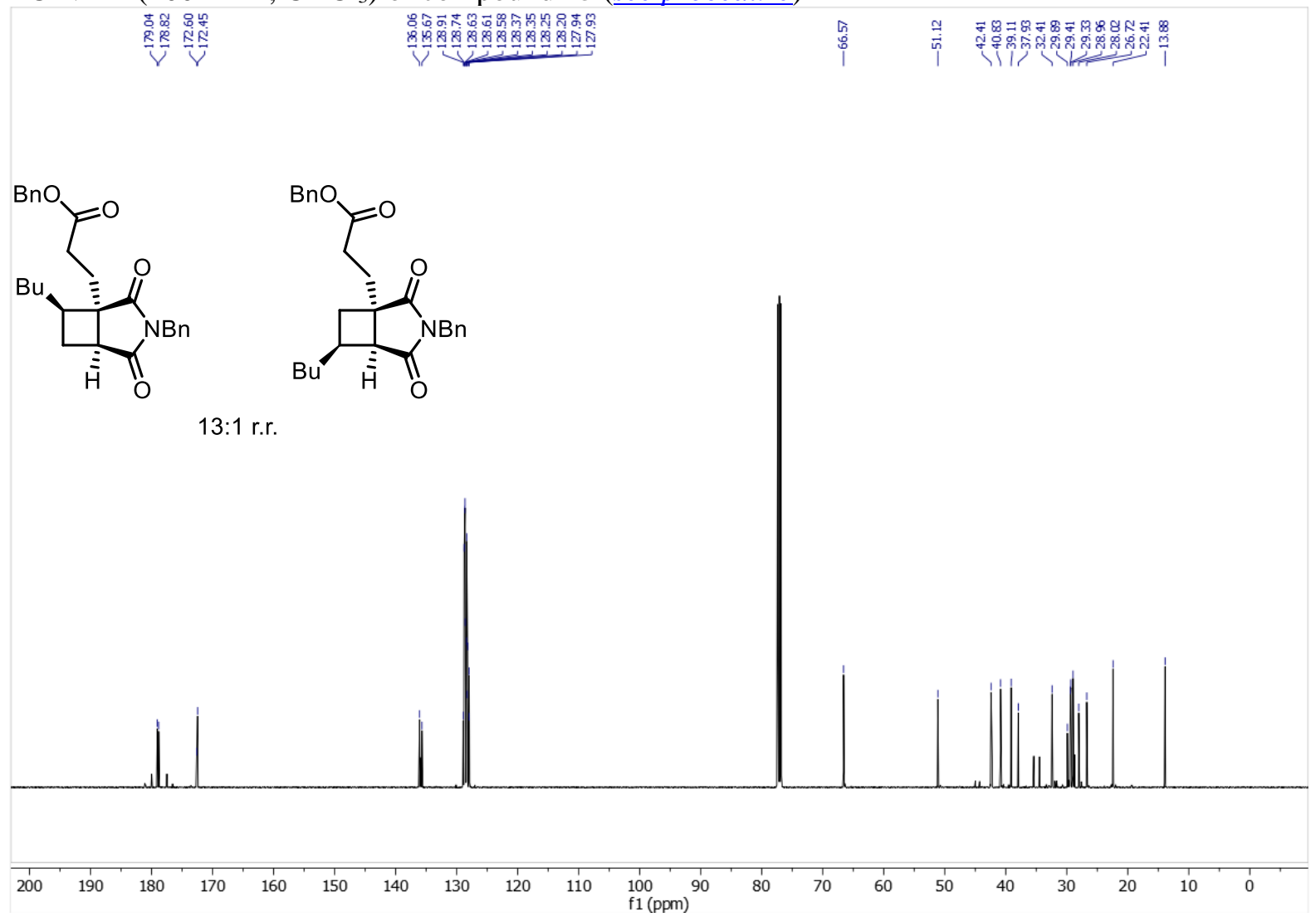


${ }^{1} \mathrm{H} \mathrm{NMR}\left(400 \mathrm{MHz}, \mathrm{CDCl}_{3}\right.$ ) of compound 26 (see procedure)

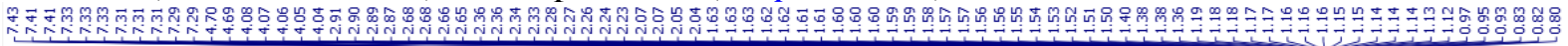

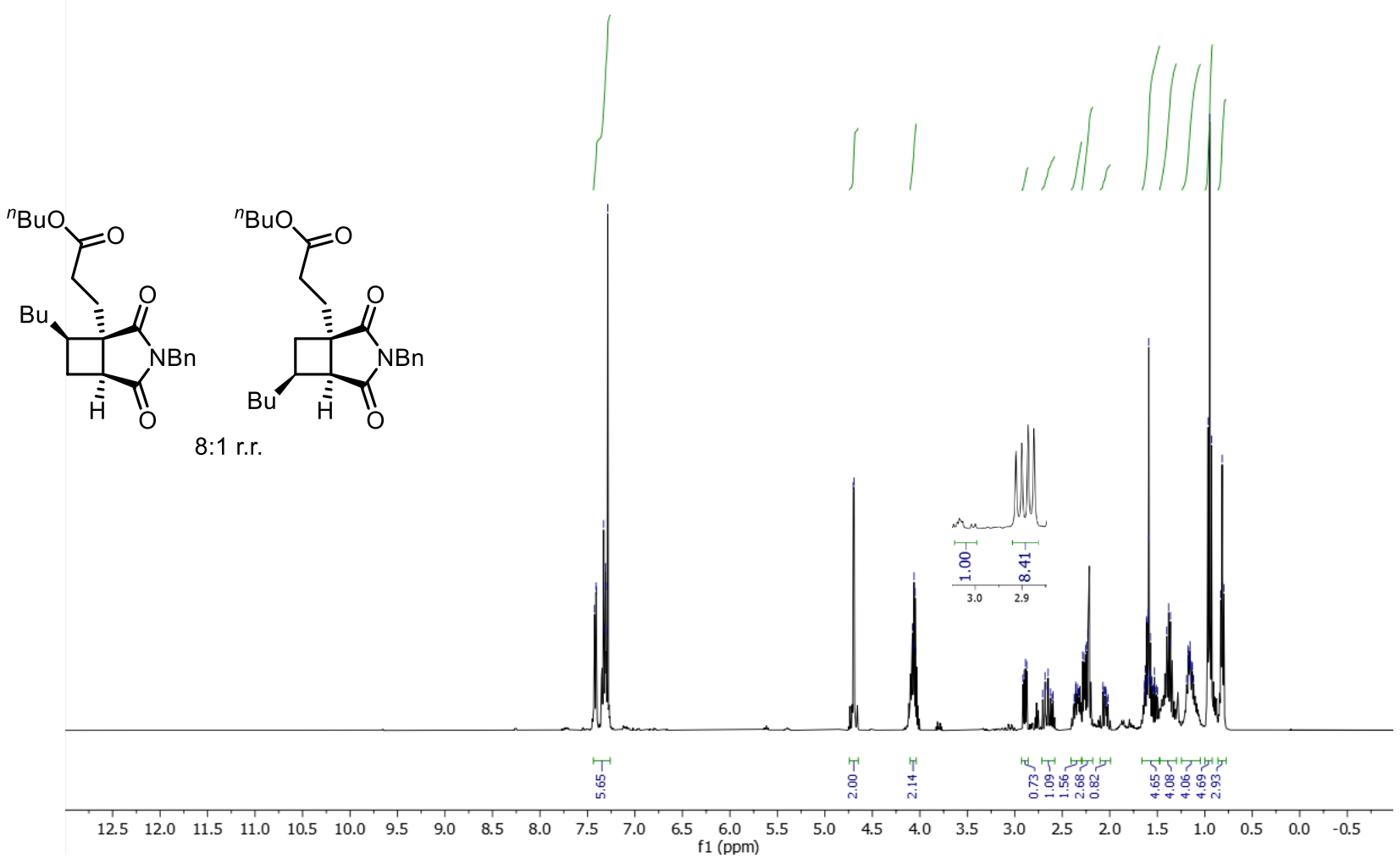

${ }^{13} \mathrm{C} \mathrm{NMR}\left(100 \mathrm{MHz}, \mathrm{CDCl}_{3}\right.$ ) of compound 26 (see procedure)

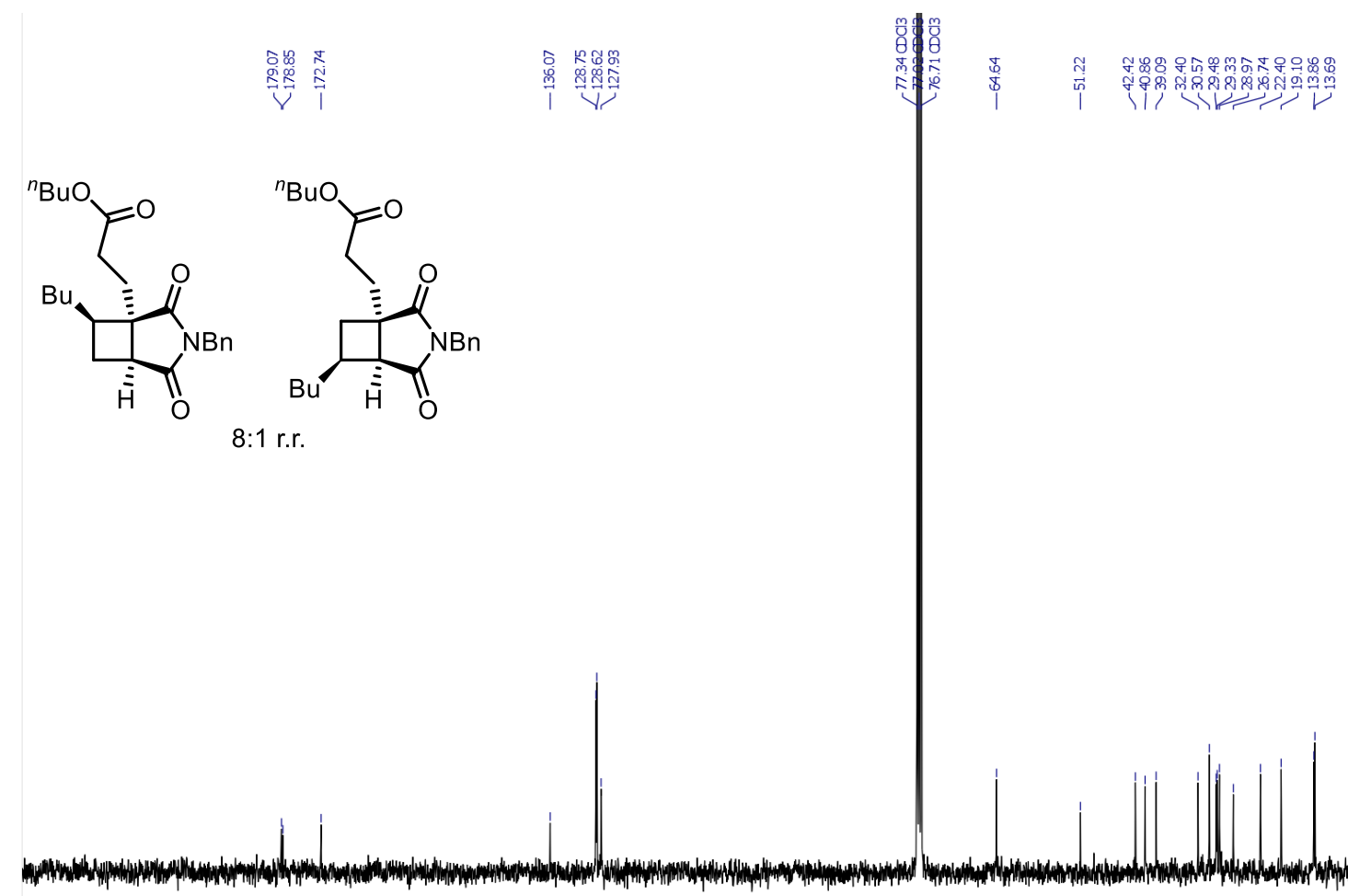

$\begin{array}{lllllllllllll}20 & 210 & 200 & 190 & 180 & 170 & 160 & 150 & 140 & 130 & 120 & 110 & 100 \\ \mathrm{f} 1(\mathrm{ppm})\end{array}$ 
${ }^{1} \mathrm{H}$ NMR (400 MHz, $\mathrm{CDCl}_{3}$ ) of compound 27 (see procedure)

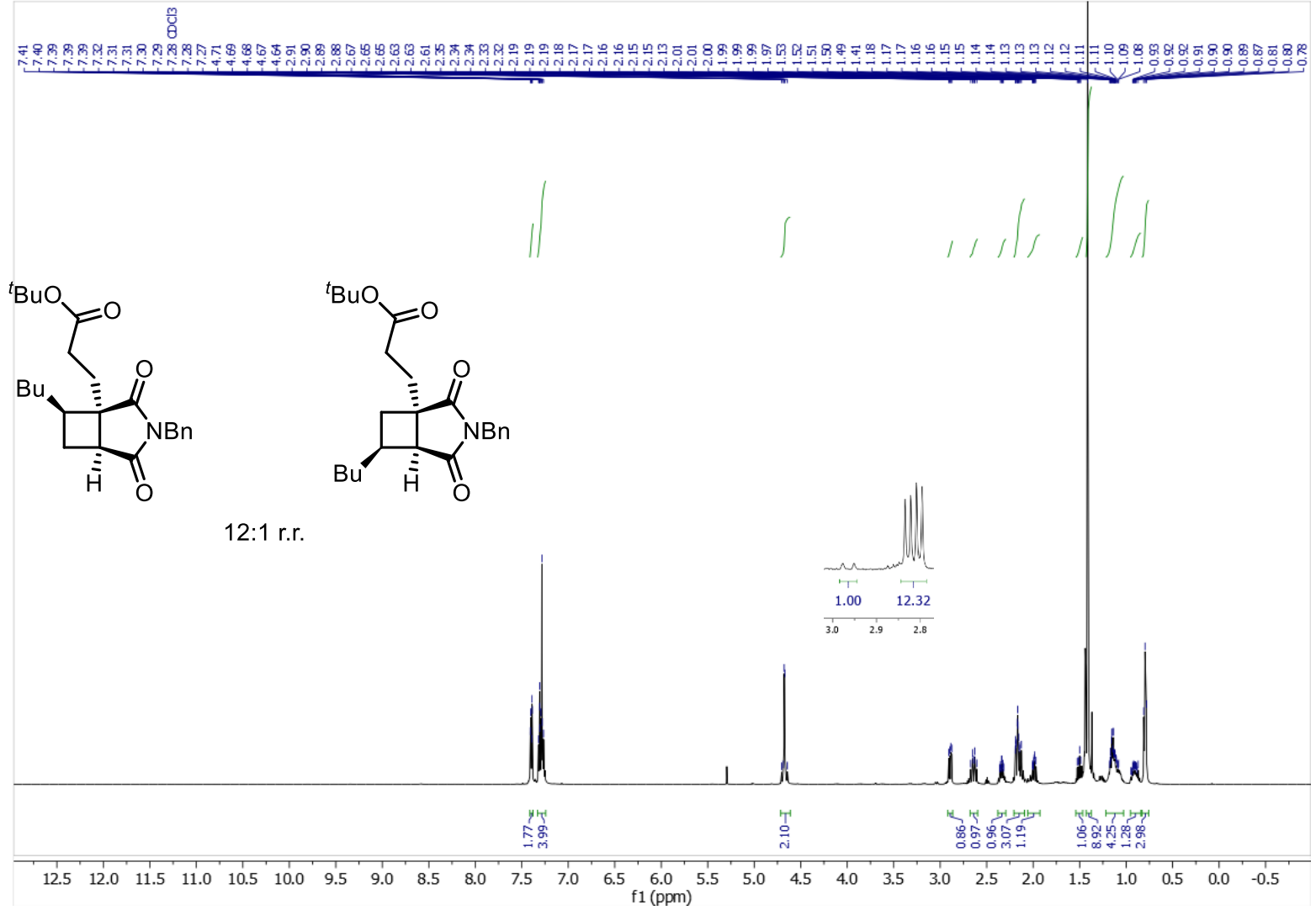

${ }^{13} \mathrm{C}$ NMR (100 MHz, $\mathrm{CDCl}_{3}$ ) of compound 27 (see procedure)

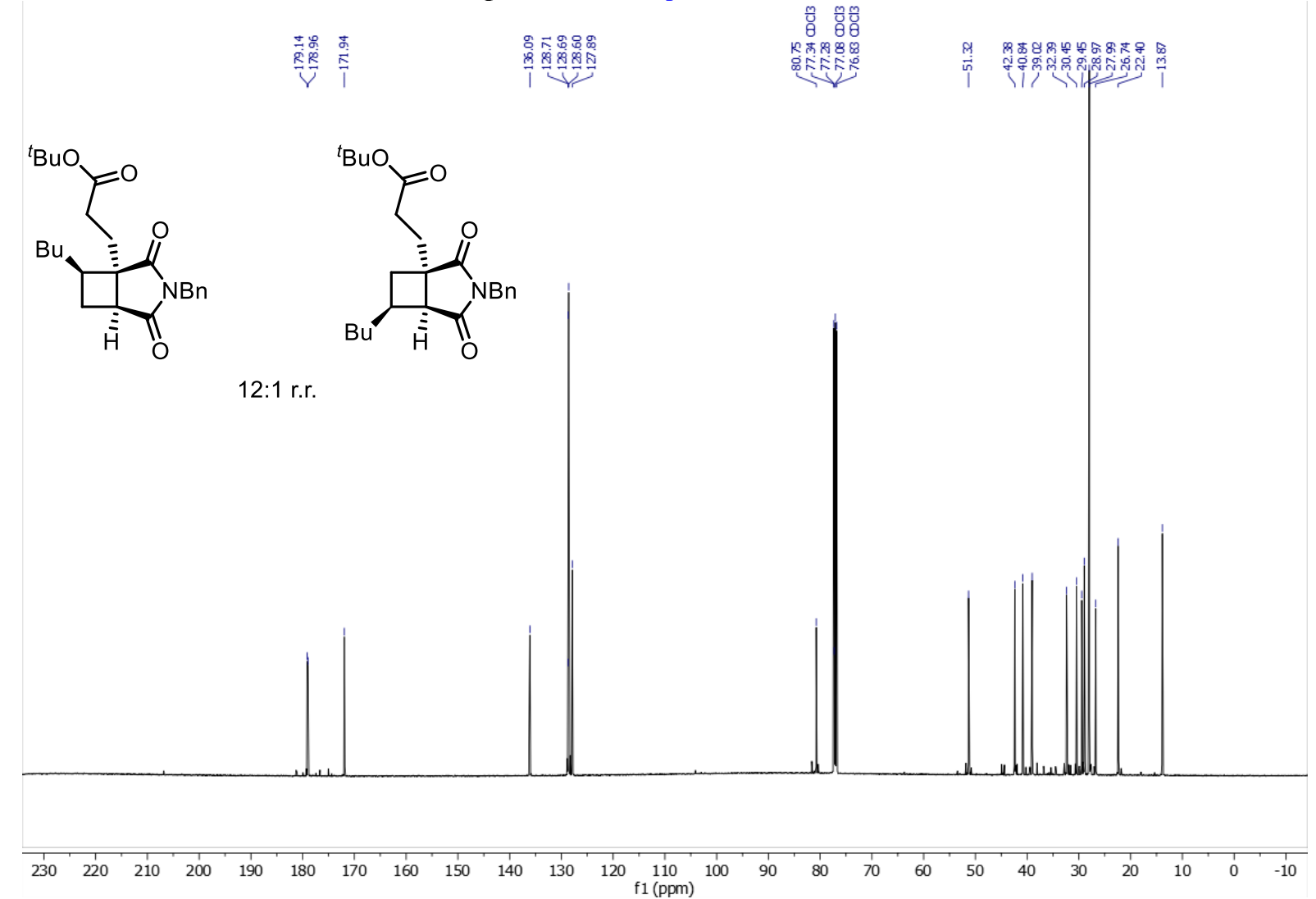


${ }^{1} \mathrm{H}$ NMR (400 MHz, $\mathrm{CDCl}_{3}$ ) of compound 28 (see procedure)

$$
\text { 学 }
$$

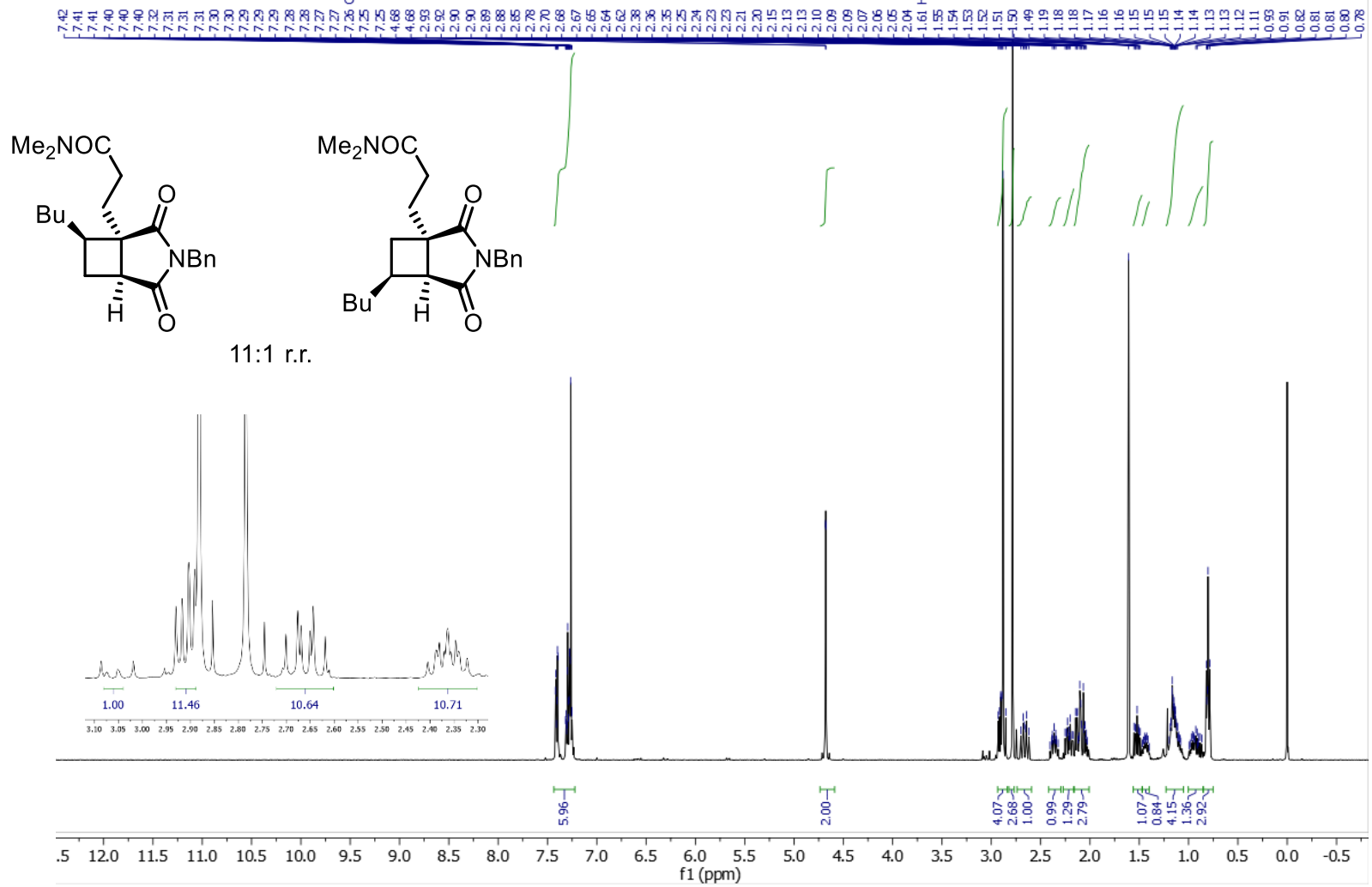

${ }^{13} \mathrm{C}$ NMR $\left(100 \mathrm{MHz}, \mathrm{CDCl}_{3}\right.$ ) of compound 28 (see procedure)

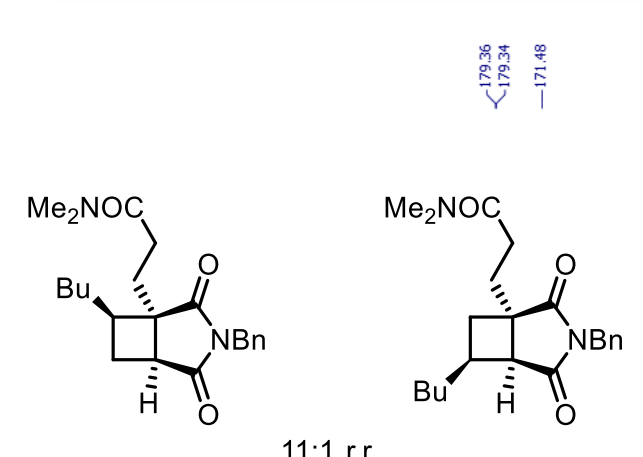

$11: 1$ r.r.
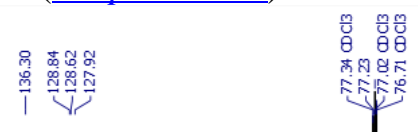

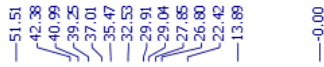

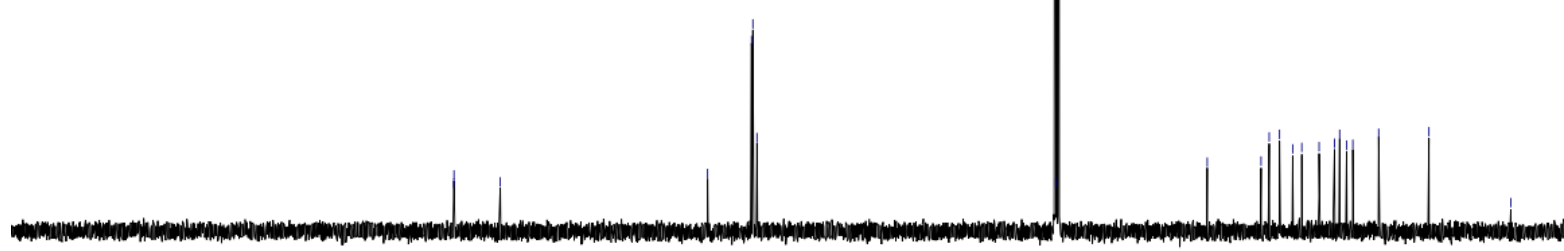

\begin{tabular}{llllllllllllllllllllllllllllllll}
\hline & 250 & 240 & 230 & 220 & 210 & 200 & 190 & 180 & 170 & 160 & 150 & 140 & 130 & 120 & 110 & 100 & 90 & 80 & 70 & 60 & 50 & 40 & 30 & 20 & 10 & 0 & 1
\end{tabular} 
${ }^{1} \mathrm{H}$ NMR (400 MHz, $\mathrm{CDCl}_{3}$ ) of compound 29 (see procedure)

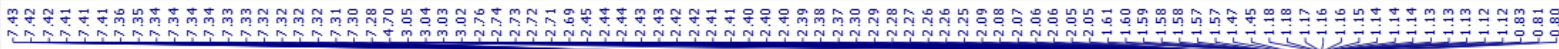

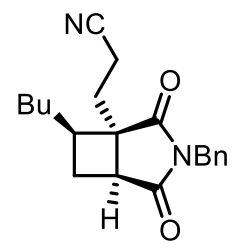

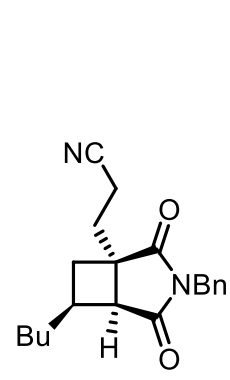

$12: 1$ r.r.
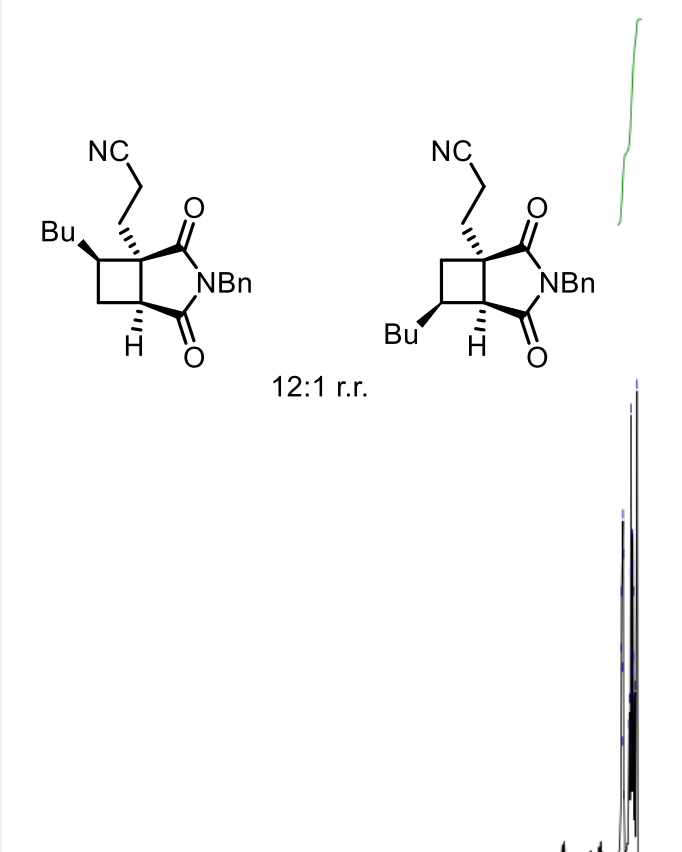

Lell
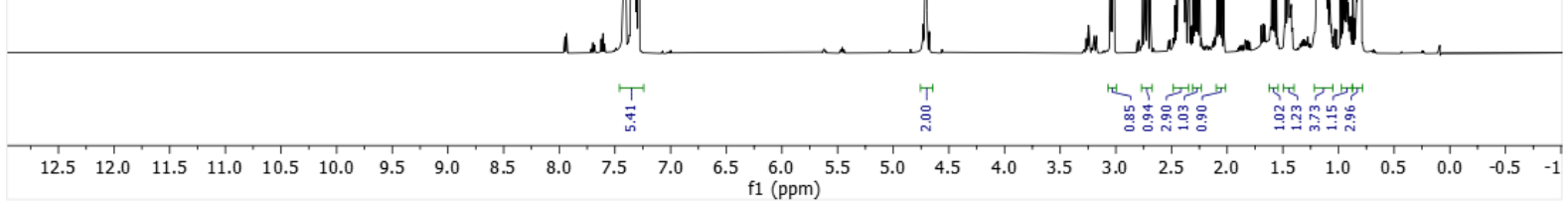

${ }^{13} \mathrm{C}$ NMR (100 MHz, $\mathrm{CDCl}_{3}$ ) of compound 29 (see procedure)
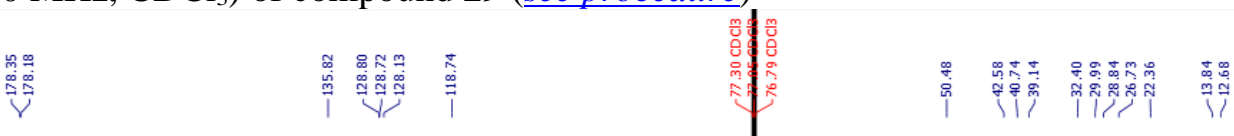

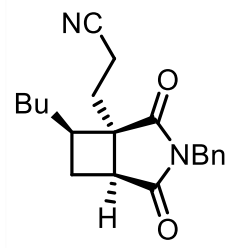<smiles></smiles>
$12: 1$ r.r.
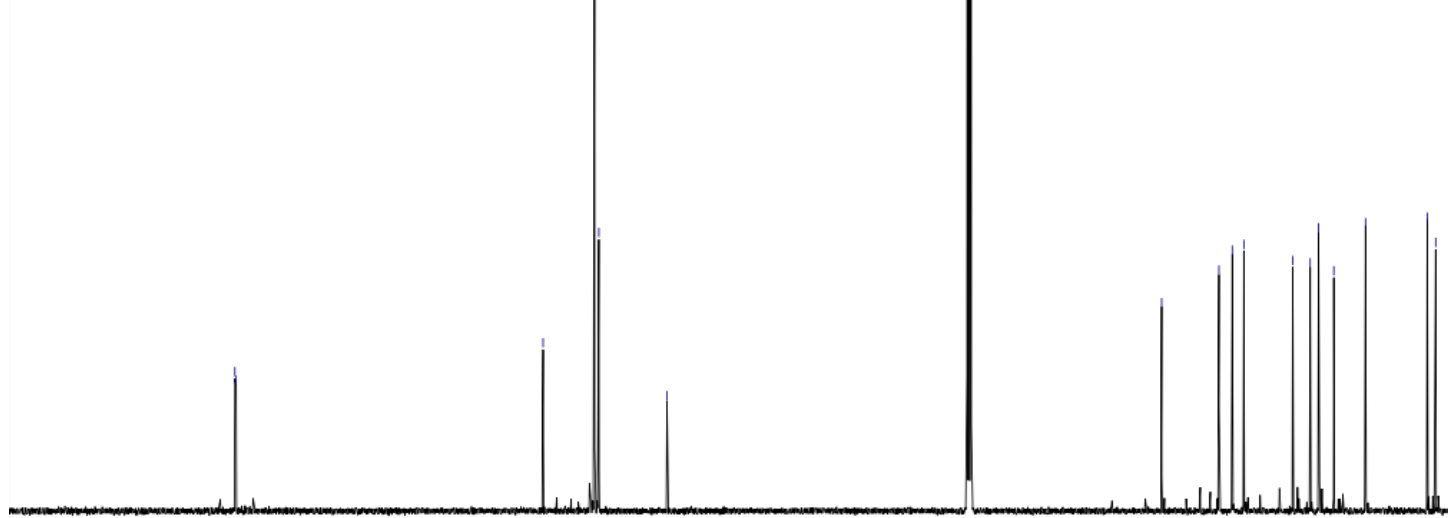

$\begin{array}{lllllllllll}200 & 190 & 180 & 170 & 160 & 150 & 140 & 130 & 120 & 110 & 100\end{array}$ 
${ }^{1} \mathrm{H}$ NMR (400 MHz, $\mathrm{CDCl}_{3}$ ) of compound 30 (see procedure) 萡

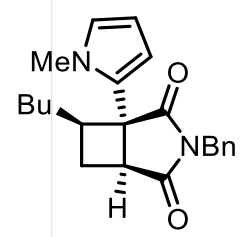

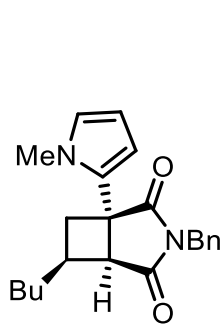

13:1 r.r.
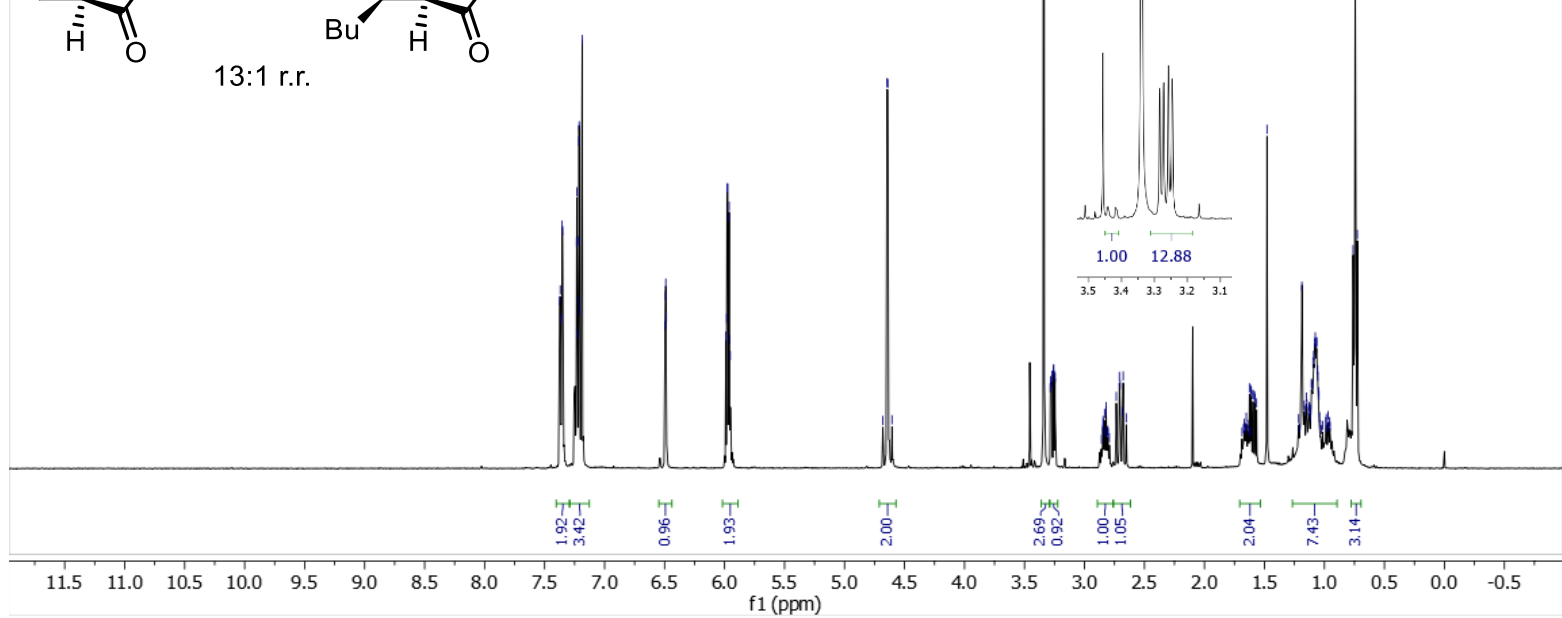

${ }^{13} \mathrm{C}$ NMR (100 MHz, $\mathrm{CDCl}_{3}$ ) of compound $\mathbf{3 0}$ (see procedure)
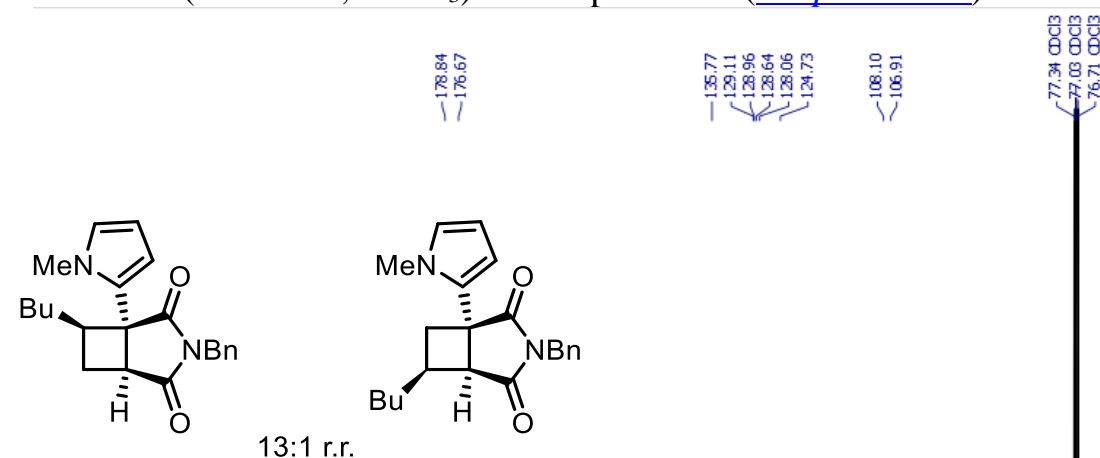

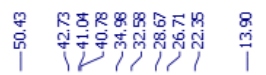

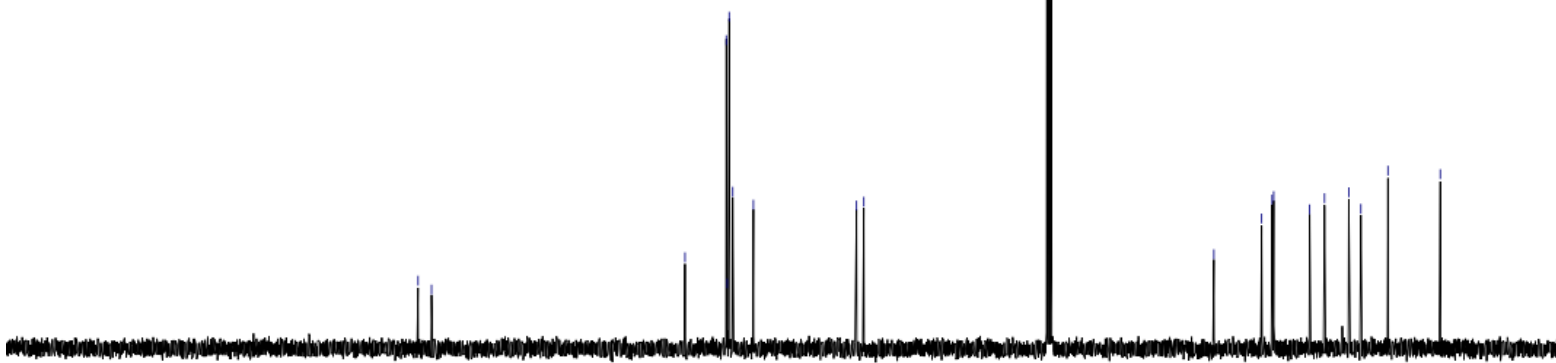

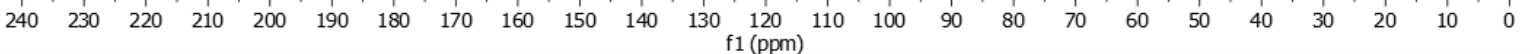


${ }^{1} \mathrm{H}$ NMR $\left(400 \mathrm{MHz}, \mathrm{CDCl}_{3}\right.$ ) of compound 31 (see procedure)

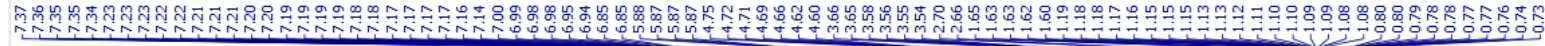

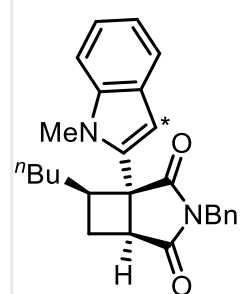

$60: 40 \mathrm{C} 2: \mathrm{C} 3$

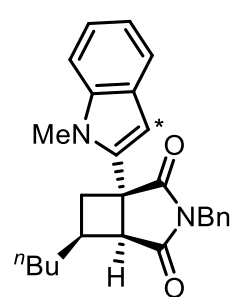

$20: 1$ r.r
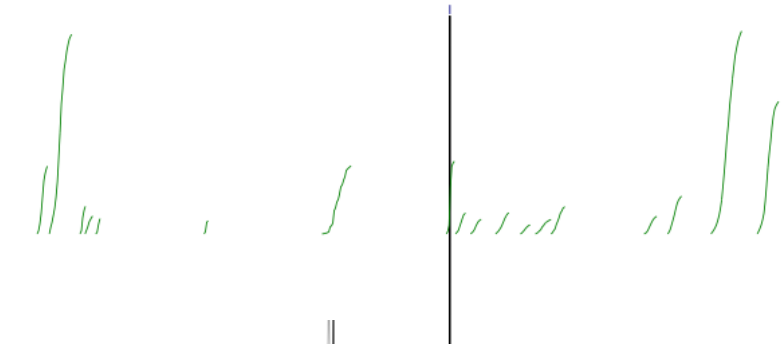

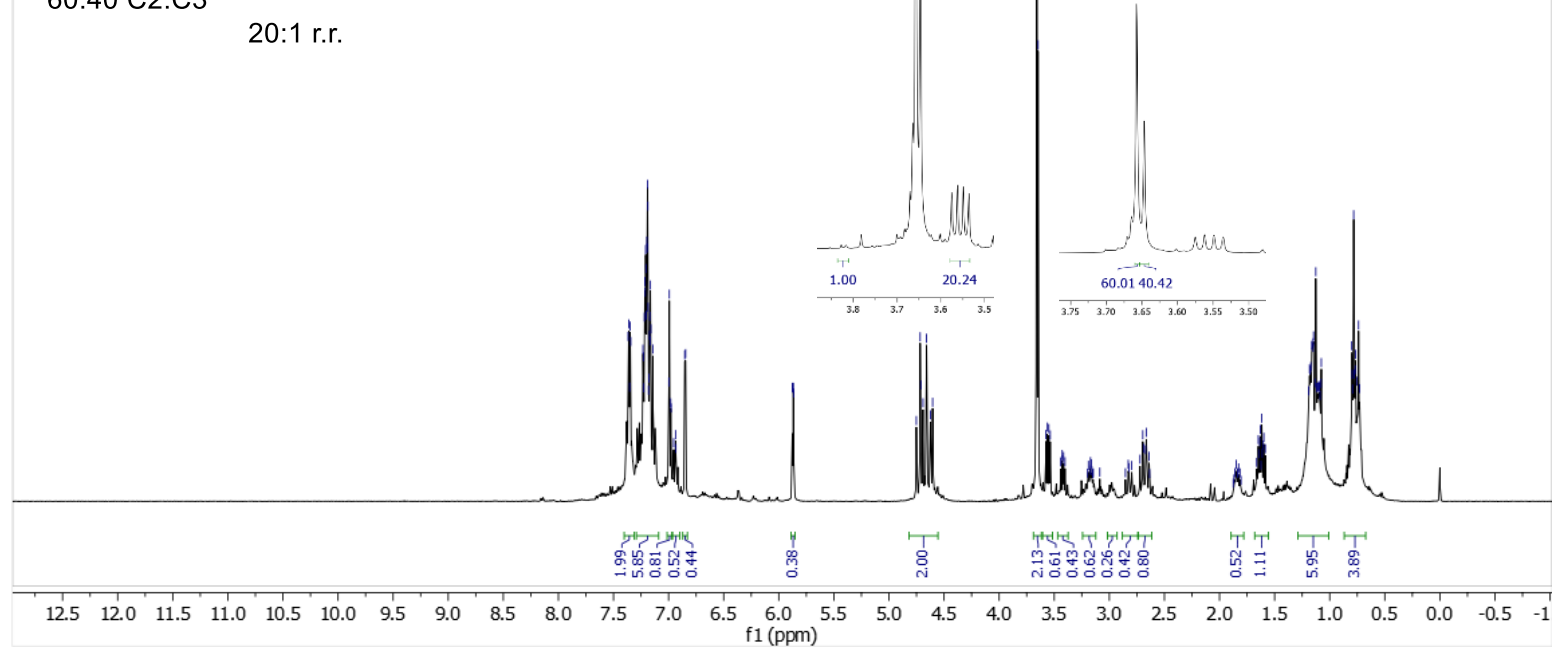

${ }^{13} \mathrm{C} \mathrm{NMR}\left(100 \mathrm{MHz}, \mathrm{CDCl}_{3}\right.$ ) of compound 31 (see procedure)
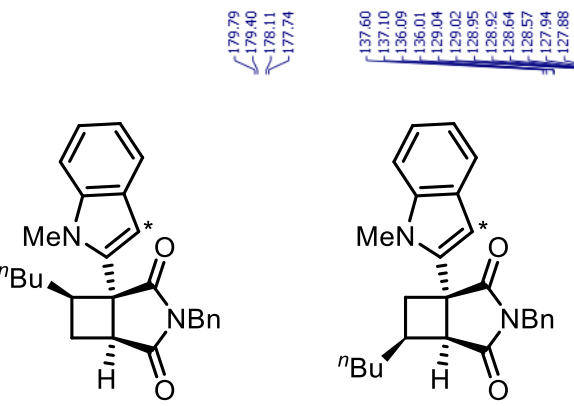

$60: 40 \mathrm{C2}: \mathrm{C3}$

$20: 1$ r.r.

$\begin{array}{lllllllllll}210 & 200 & 190 & 180 & 170 & 160 & 150 & 140 & 130 & 120 & \begin{array}{l}110 \\ \mathrm{f} 1(\mathrm{ppm})\end{array}\end{array}$

R०F

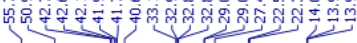
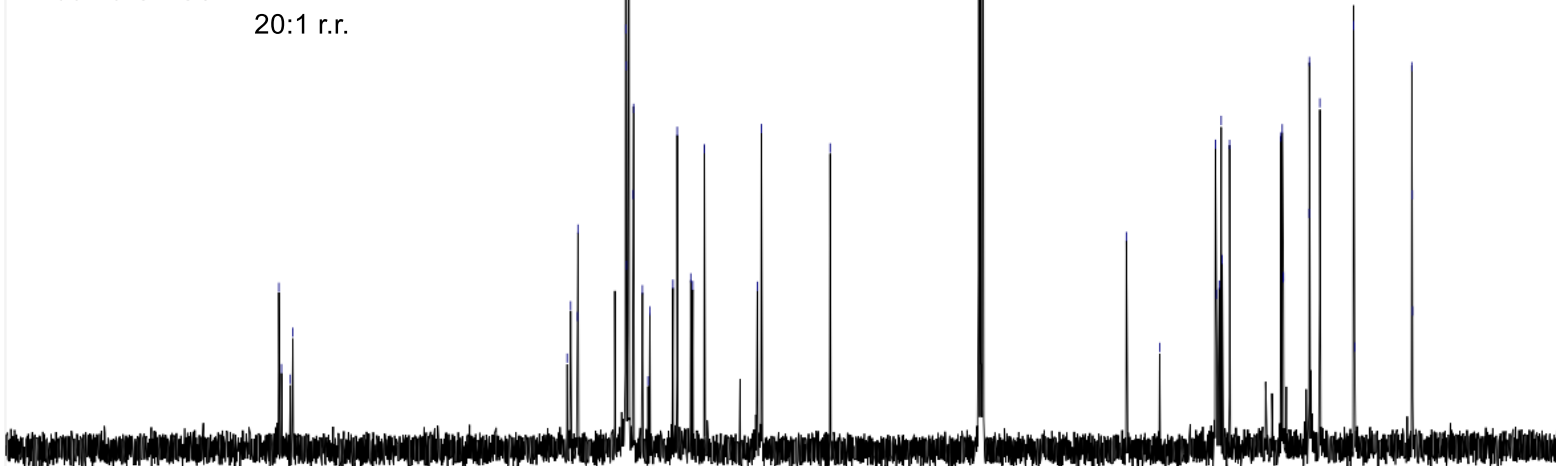
${ }^{1} \mathrm{H}$ NMR (400 MHz, $\mathrm{CDCl}_{3}$ ) of compound 32 (see procedure)

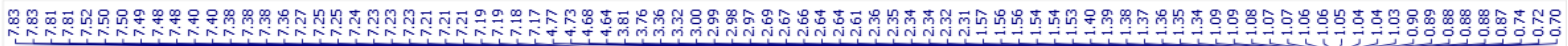

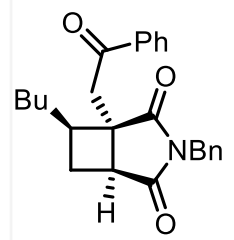
$8: 1$ r.r.

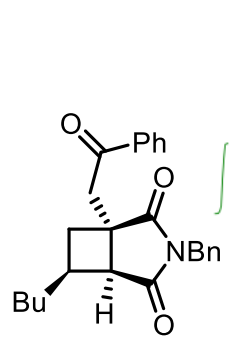<smiles>C#CCCCCCCC</smiles>

$$
\text { (1) }
$$

8.1 r.
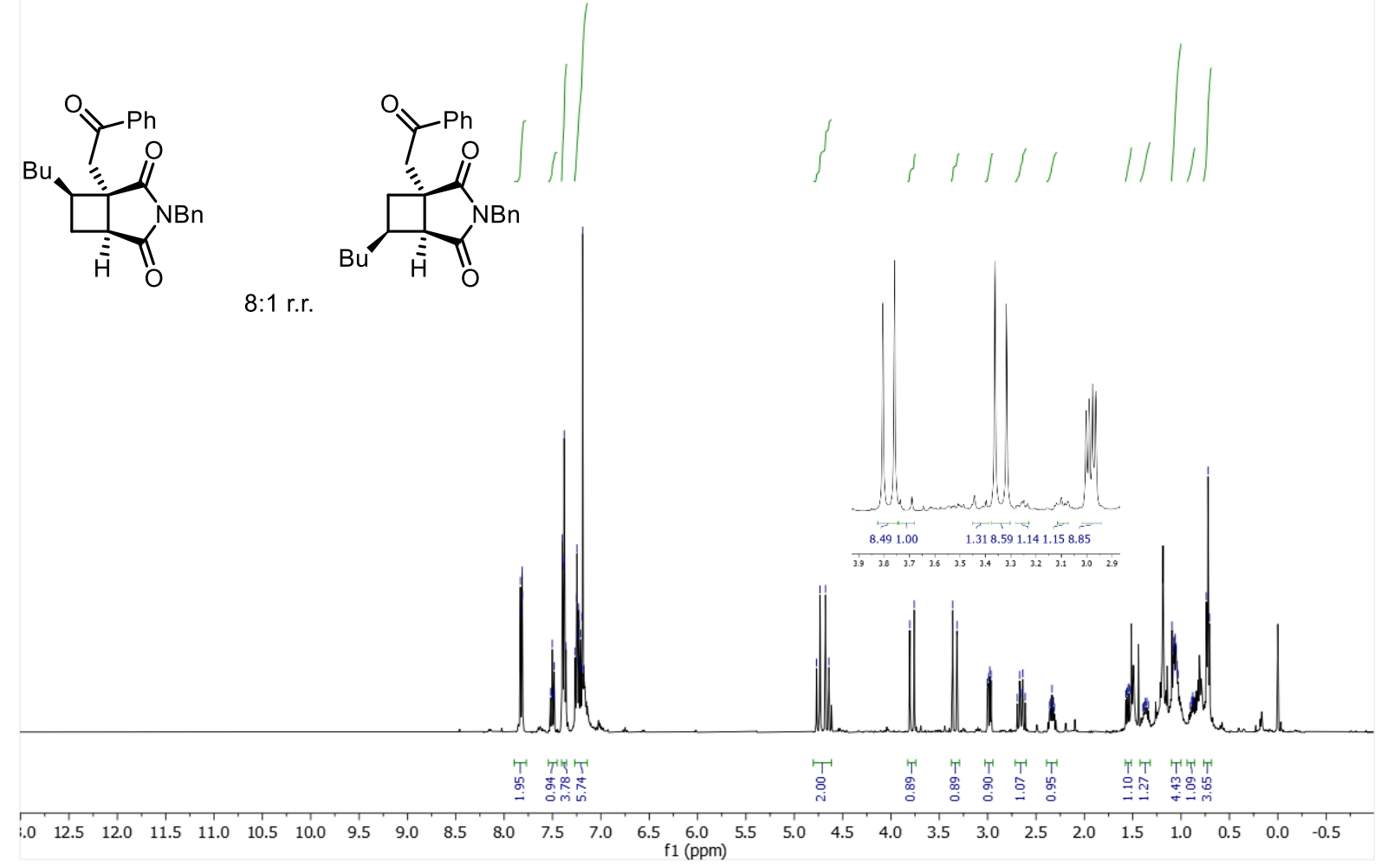

${ }^{13} \mathrm{C}$ NMR (100 MHz, $\left.\mathrm{CDCl}_{3}\right)$ of compound 32 (see procedure)
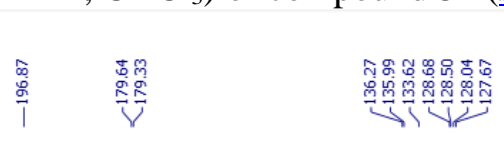<smiles>O=C(CC1C(=O)N(Br)C(=O)C1CC(Br)Br)c1ccccc1</smiles>

$8: 1$ r.r.

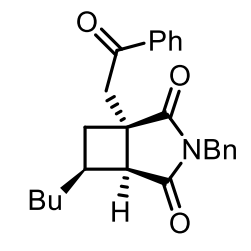

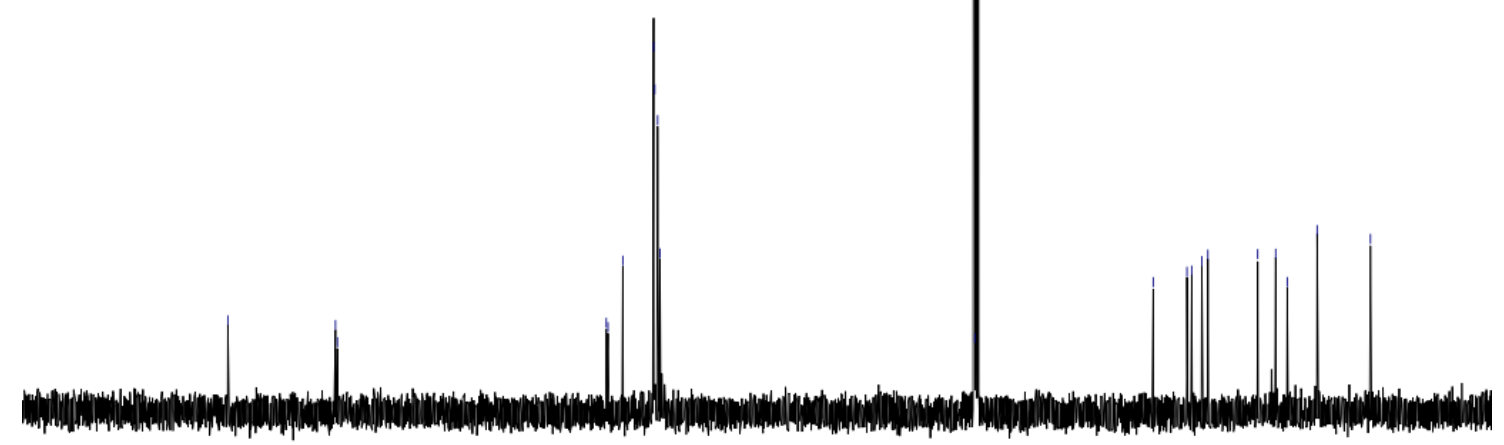

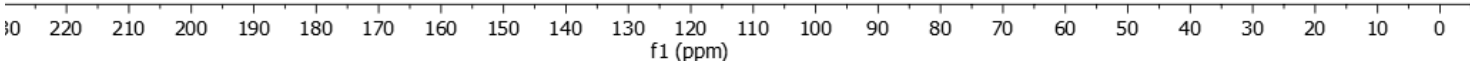


${ }^{1} \mathrm{H} \mathrm{NMR}\left(400 \mathrm{MHz}, \mathrm{CDCl}_{3}\right.$ ) of compound 33 (see procedure)

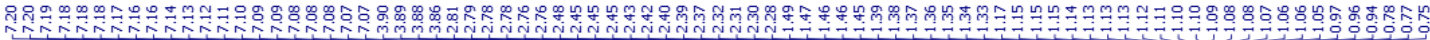
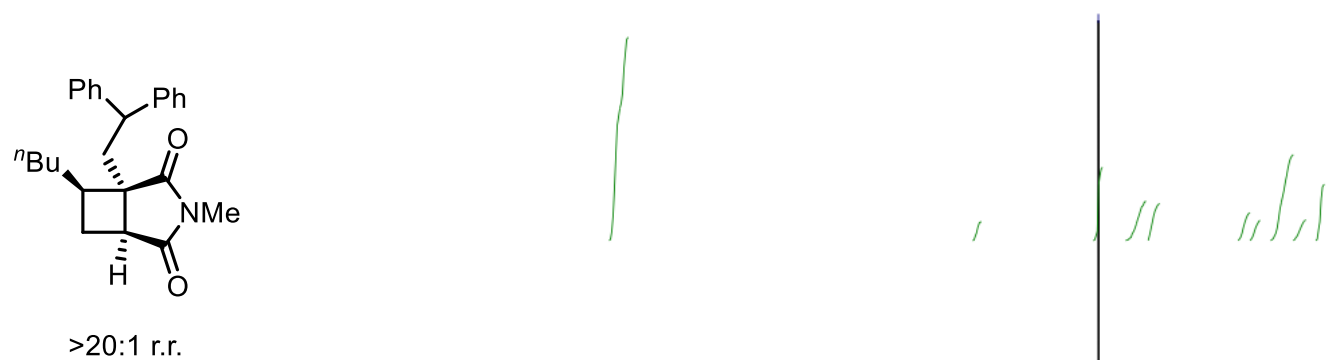

$>20: 1$ r.r.

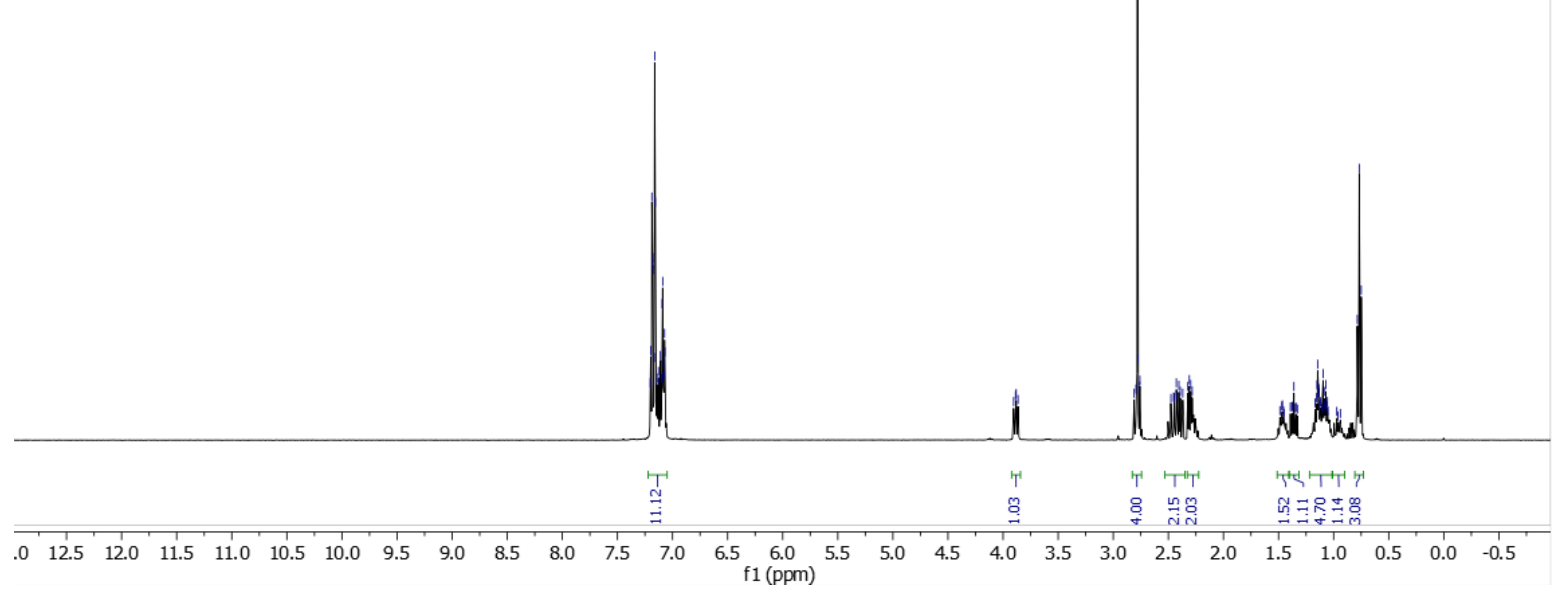

${ }^{13} \mathrm{C}$ NMR $\left(100 \mathrm{MHz}, \mathrm{CDCl}_{3}\right.$ ) of compound $\mathbf{3 3}$ (see procedure)
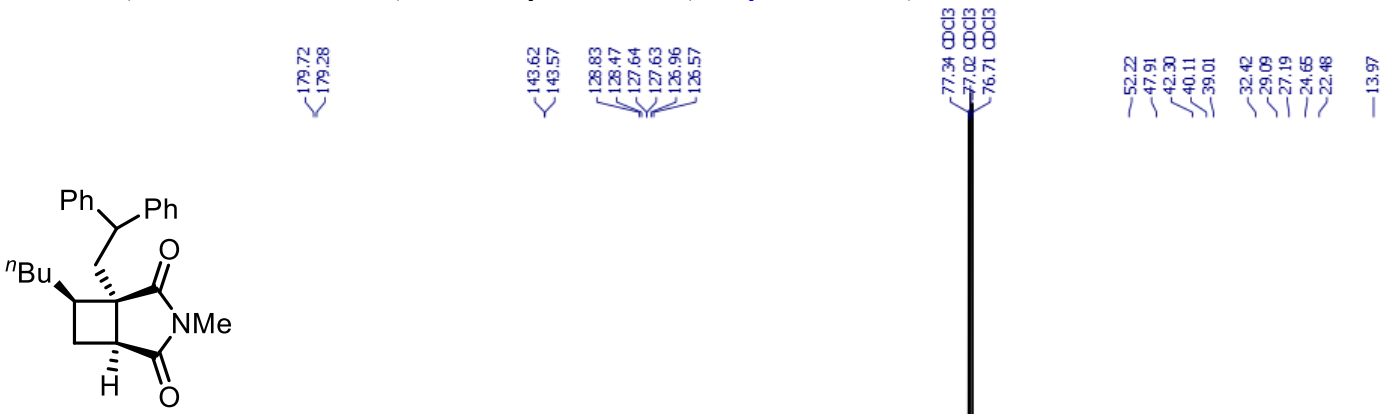

$>20: 1$ r.r.

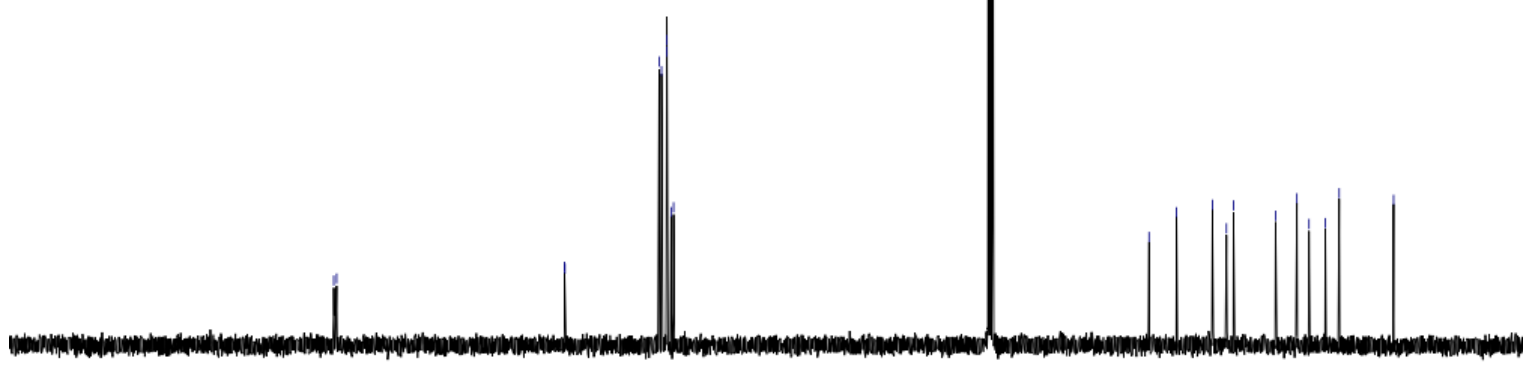

\begin{tabular}{lllllllllllllllllllllllllllll}
\hline 30 & 220 & 210 & 200 & 190 & 180 & 170 & 160 & 150 & 140 & 130 & 120 & 110 & 100 & 90 & 80 & 70 & 60 & 50 & 40 & 30 & 20 & 10 & 0
\end{tabular} 
${ }^{1} \mathrm{H}$ NMR (400 MHz, $\mathrm{CDCl}_{3}$ ) of compound $\mathbf{3 4}$ (see procedure)

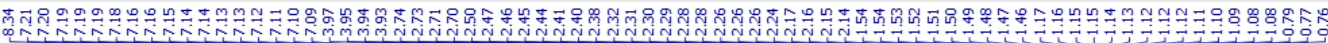

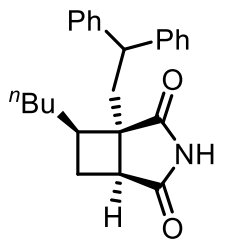

$>20: 1$ r.r.

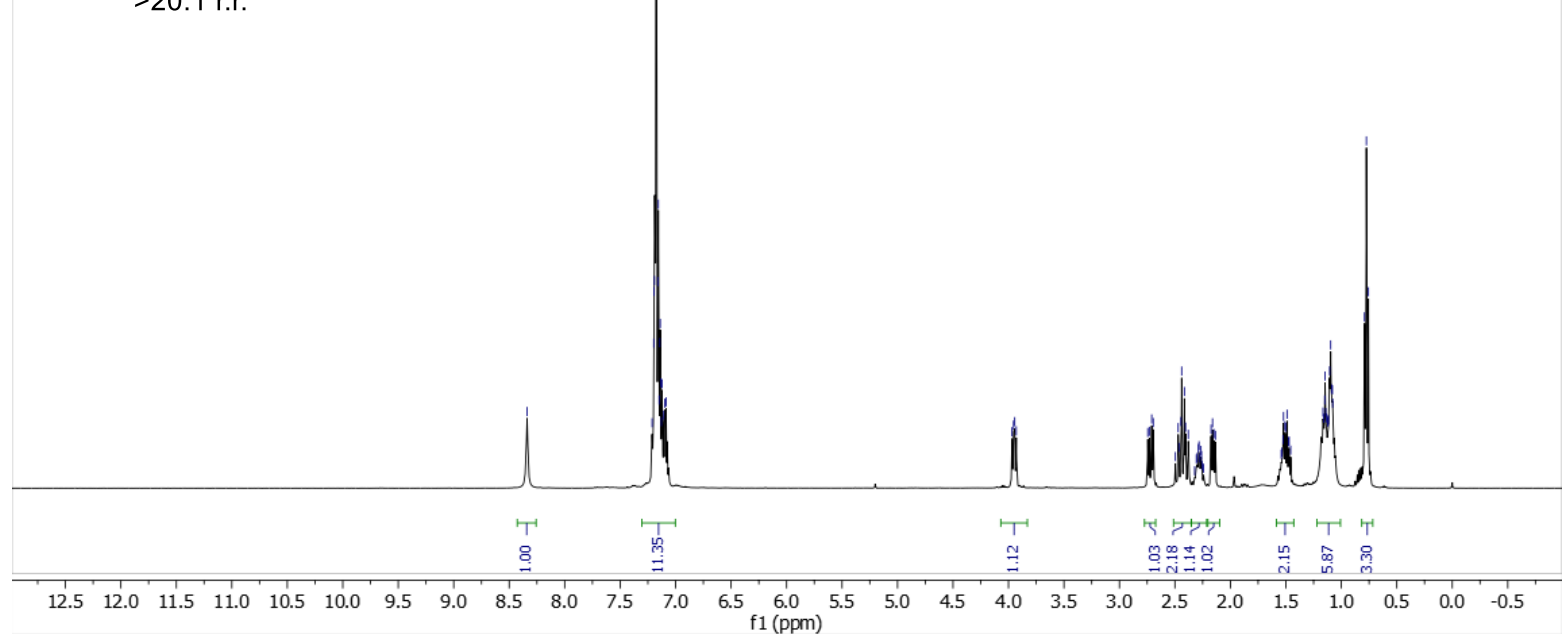

${ }^{13} \mathrm{C}$ NMR (100 MHz, $\mathrm{CDCl}_{3}$ ) of compound 34 (see procedure)
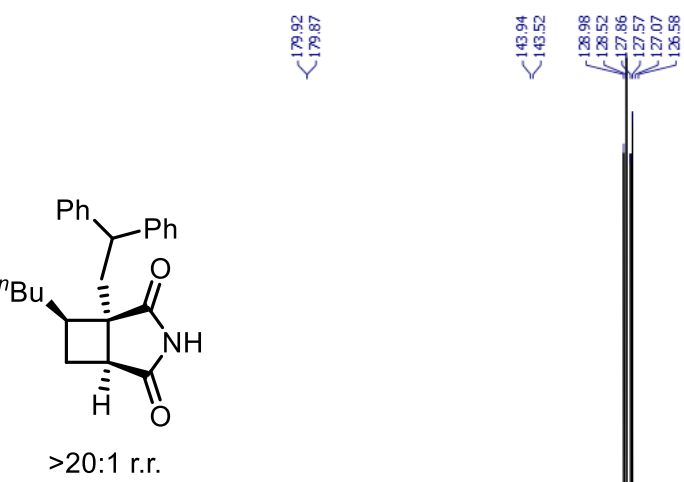

$>20: 1$ r.r.

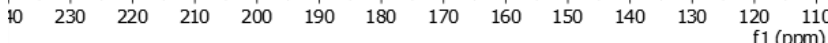


${ }^{1} \mathrm{H}$ NMR (400 MHz, $\mathrm{CDCl}_{3}$ ) of compound 35 (see procedure)

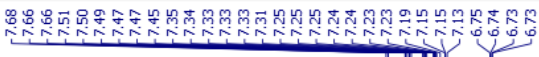
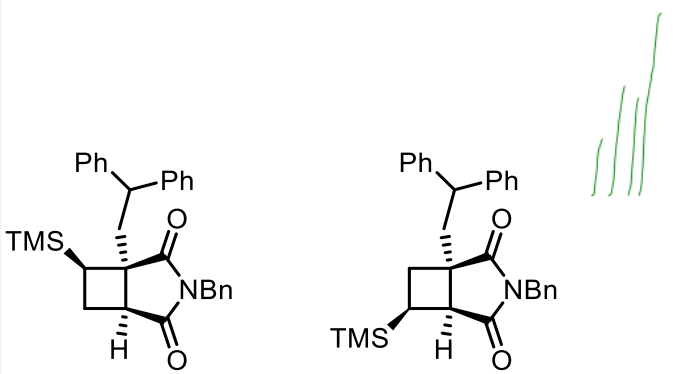

$4: 1$ r.r.
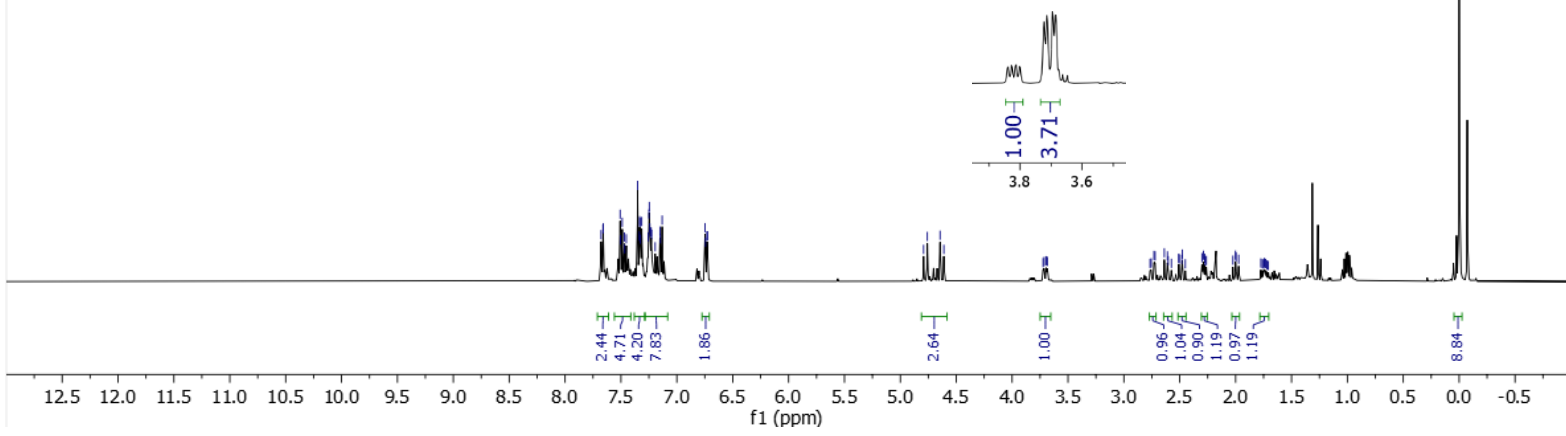

${ }^{13} \mathrm{C}$ NMR (100 MHz, $\mathrm{CDCl}_{3}$ ) of compound 35 (see procedure)

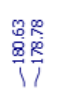<smiles></smiles><smiles>C[C@H]1C[C@H]2C(=O)N([13CH3])C(=O)[C@H]2C1c1ccccc1</smiles>
$4: 1$ r.r.

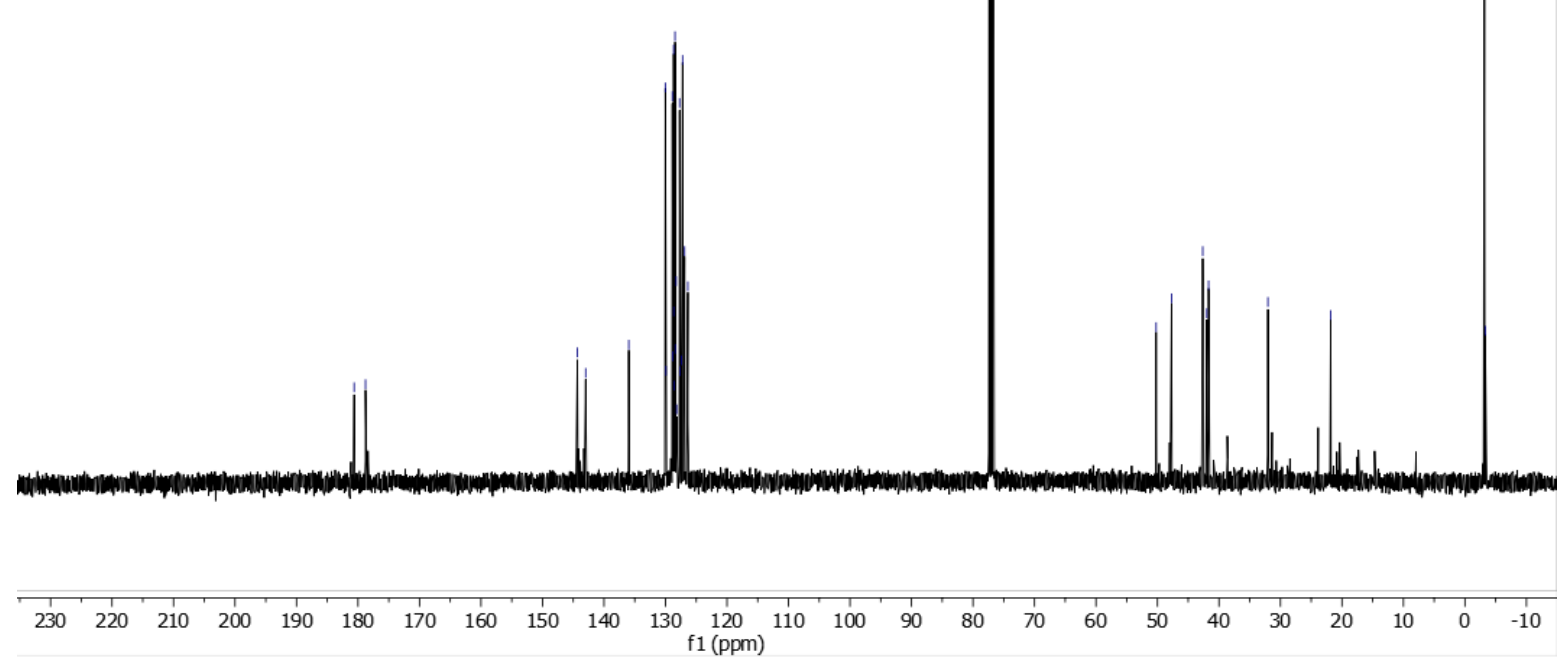


${ }^{1} \mathrm{H}$ NMR (400 MHz, $\mathrm{CDCl}_{3}$ ) of compound $\mathbf{3 6}$ (see procedure)

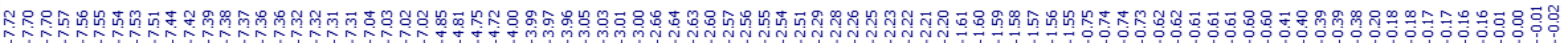<smiles>O=C1[NH2+]C(=O)[C@H]2[C@@H]1C[C@@H](C1CC1)[C@@H]2C(c1ccccc1)c1ccccc1</smiles>
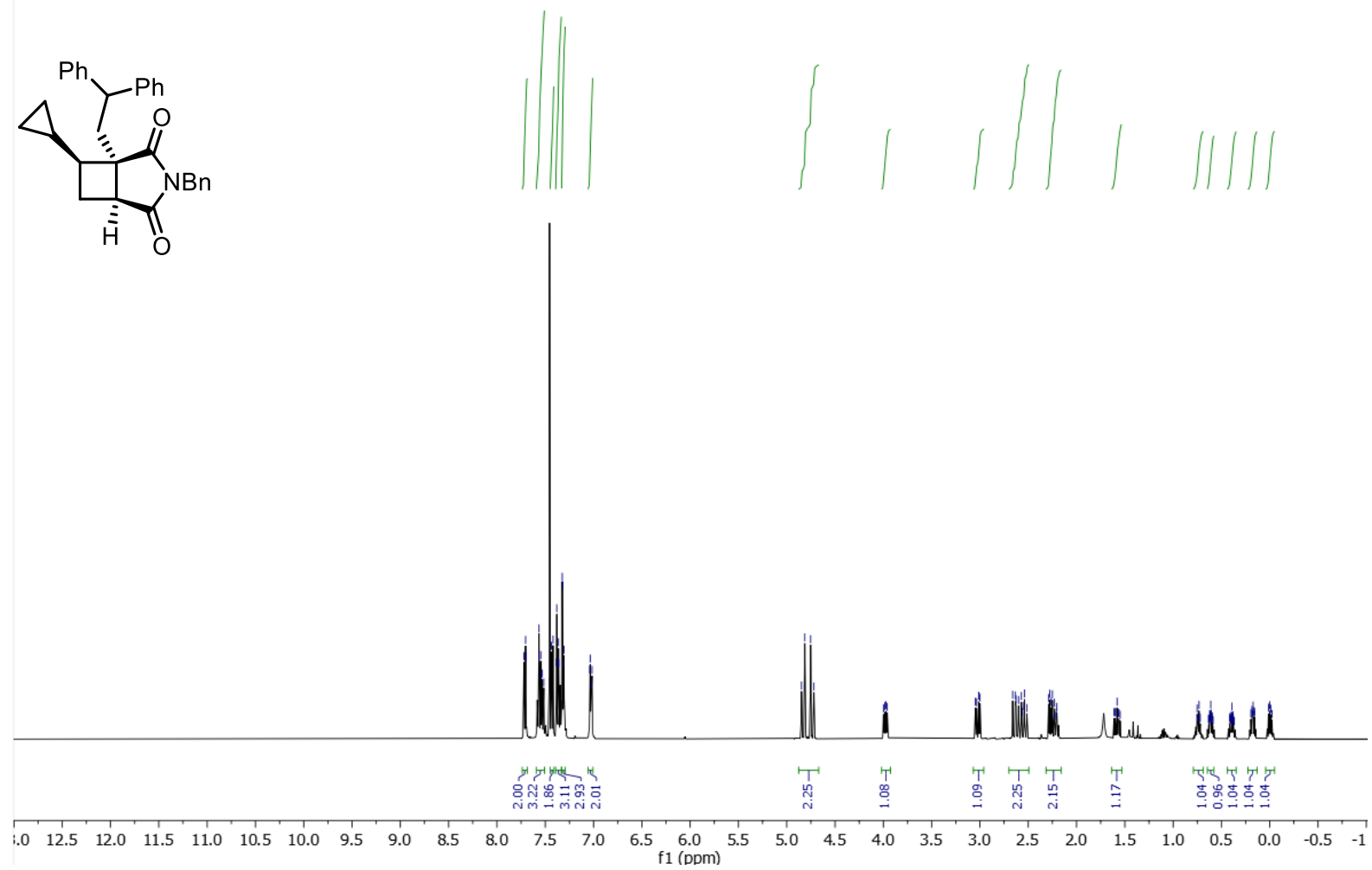

${ }^{13} \mathrm{C}$ NMR (100 MHz, $\mathrm{CDCl}_{3}$ ) of compound 36 (see procedure)<smiles>O=C1[C@H](Br)C(=O)[C@@H]2[C@@H](c3ccccc3)C(C3CC3)C[C@@H]12</smiles>

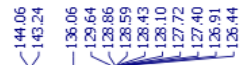
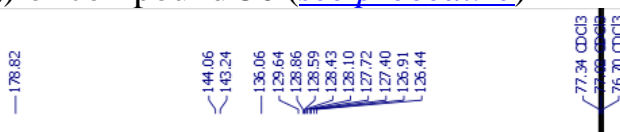

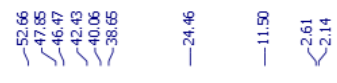

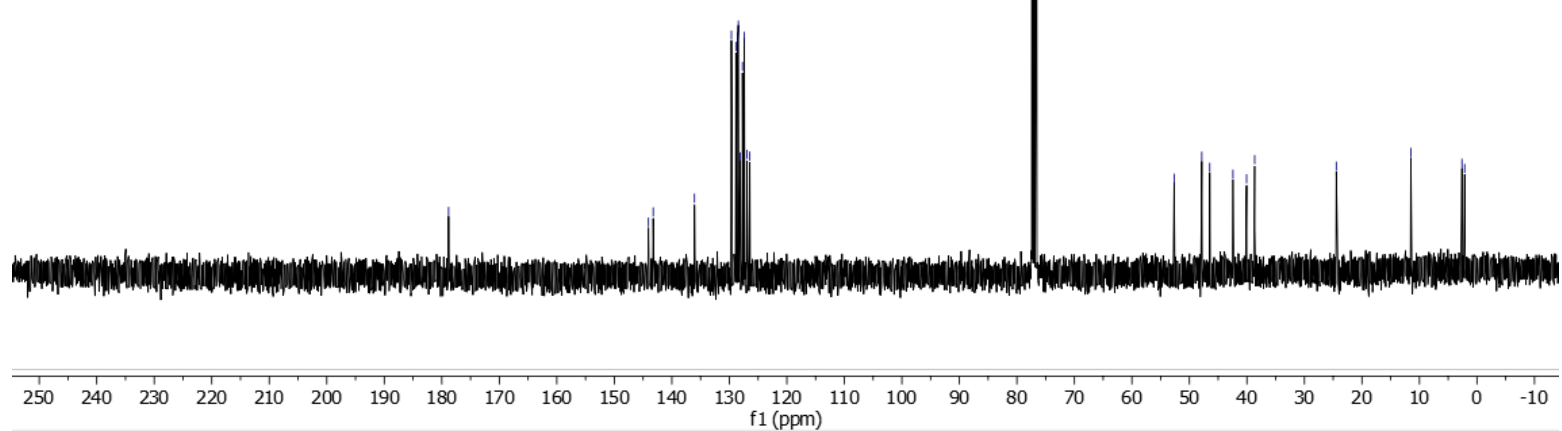


${ }^{1} \mathrm{H} \mathrm{NMR}\left(400 \mathrm{MHz}, \mathrm{CDCl}_{3}\right.$ ) of compound 37 (see procedure)

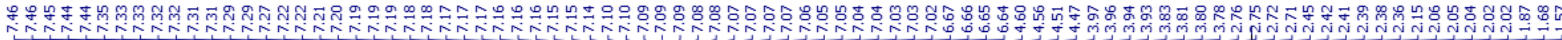

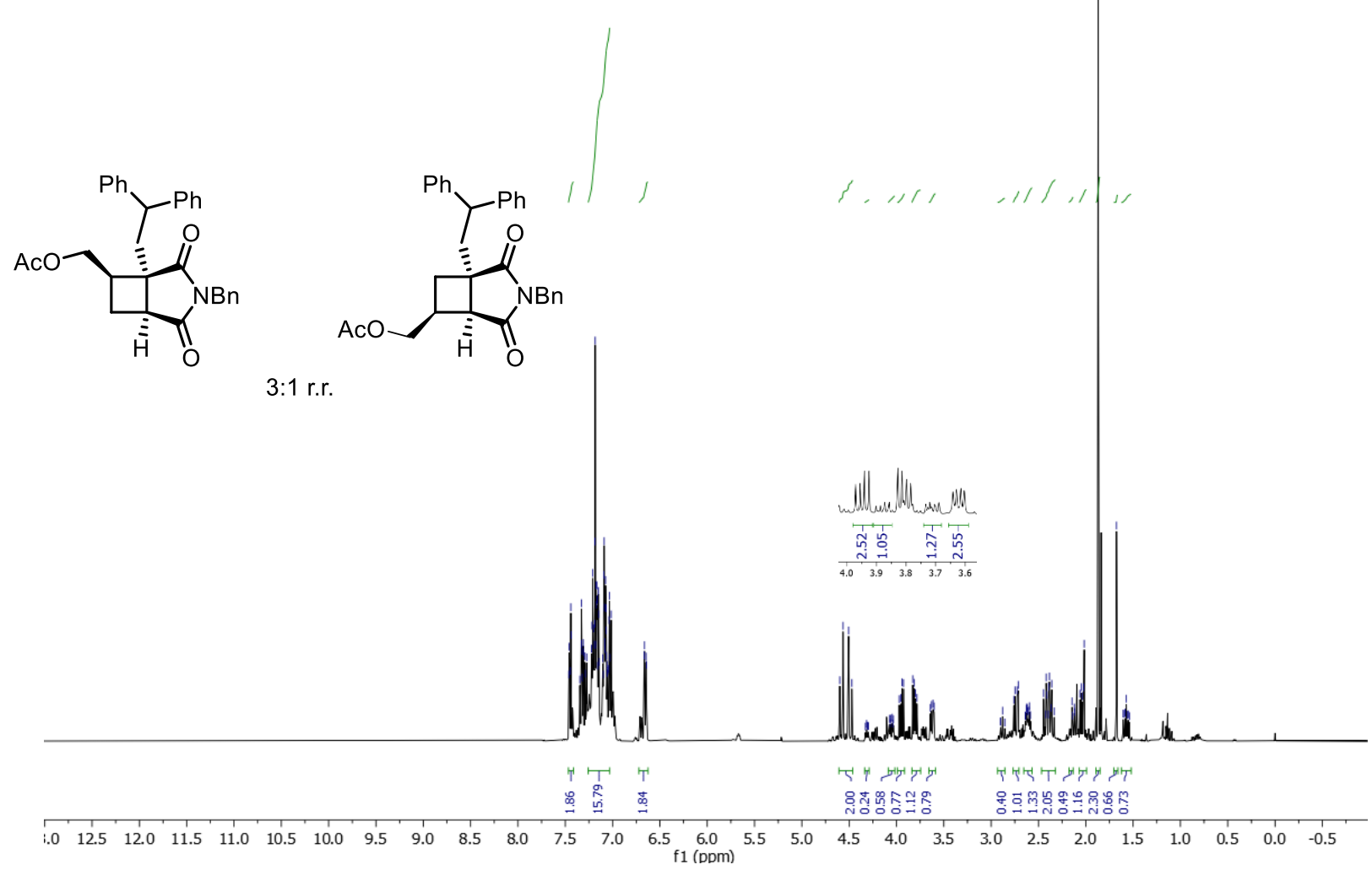

${ }^{13} \mathrm{C}$ NMR $\left(100 \mathrm{MHz}, \mathrm{CDCl}_{3}\right.$ ) of compound 37 (see procedure)

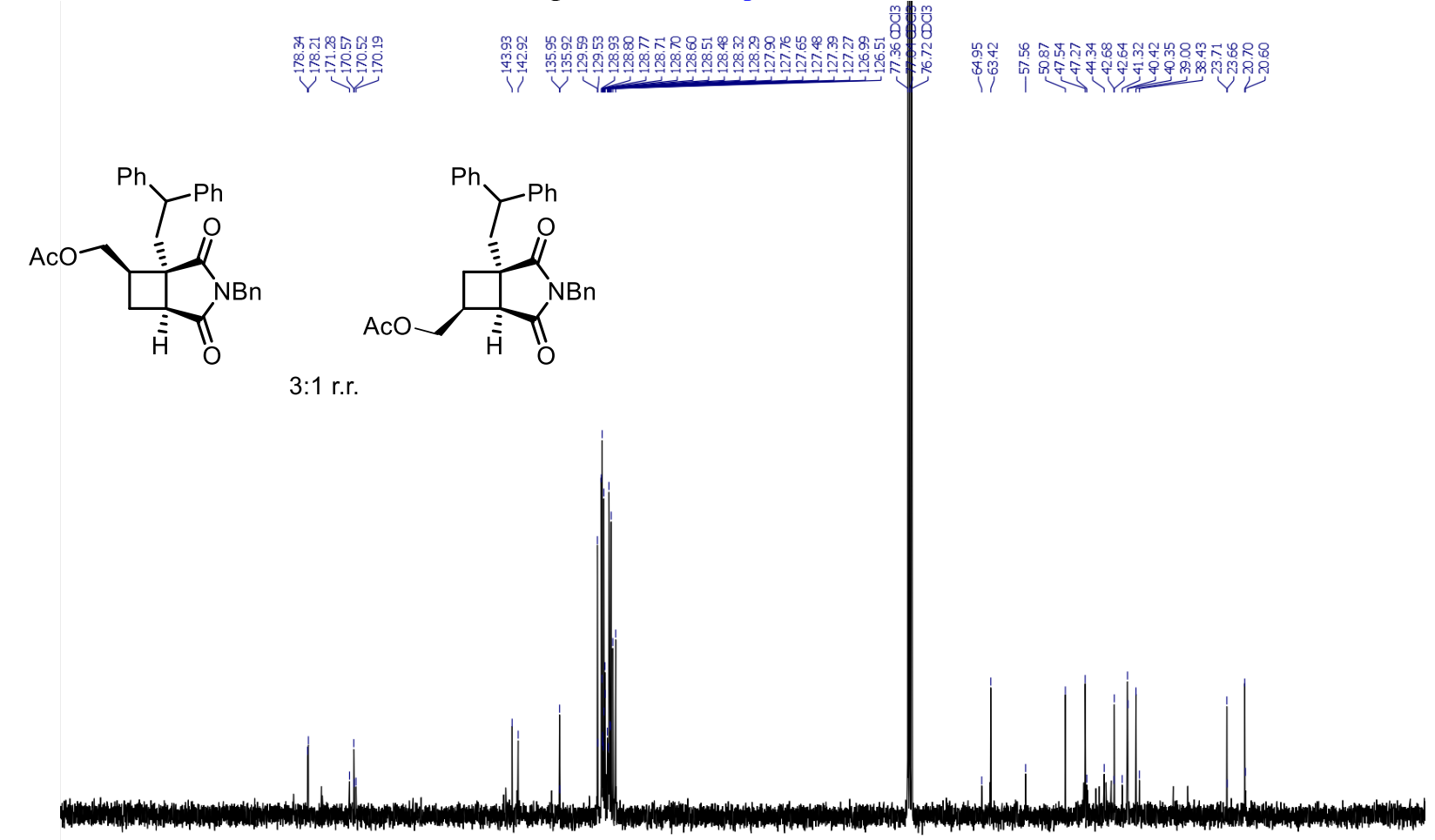

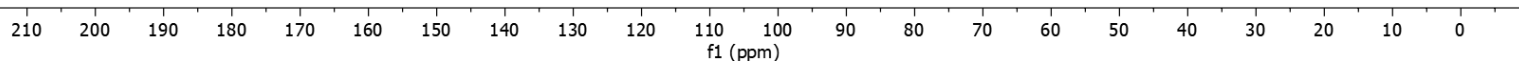


${ }^{1} \mathrm{H}$ NMR (400 MHz, $\mathrm{CDCl}_{3}$ ) of compound 38 (see procedure)

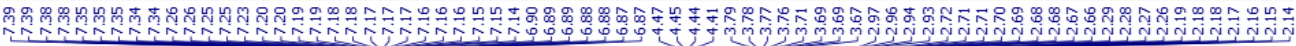<smiles>O=C1[C@H](c2ccccc2)C(=O)[C@@]2(CC(Cc3ccccc3)c3ccccc3)[C@@H]1C[C@@H]2c1ccccc1</smiles><smiles>O=C1[C@H]2C(c3ccccc3)C[C@]2(CC(c2ccccc2)c2ccccc2)C(=O)N1Cc1ccccc1</smiles>

$6: 1$ r.r.
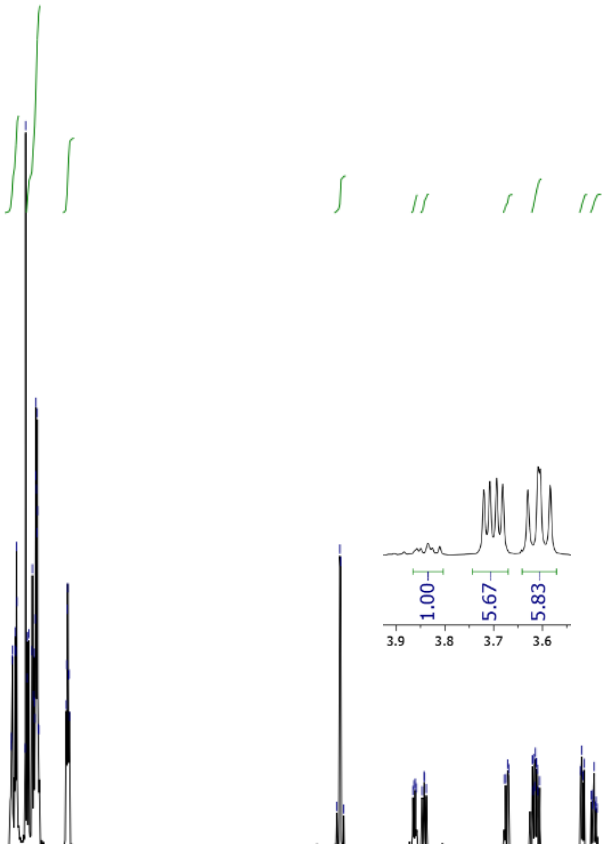

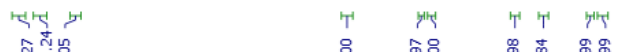

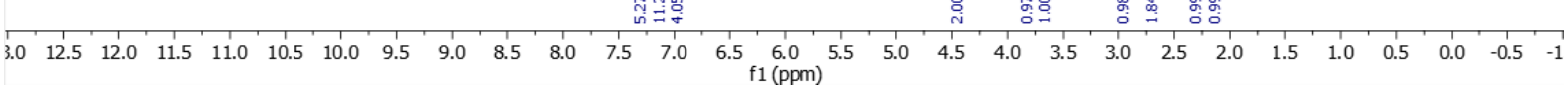

${ }^{13} \mathrm{C}$ NMR $\left(100 \mathrm{MHz}, \mathrm{CDCl}_{3}\right.$ ) of compound 38 (see procedure)

㢳誉

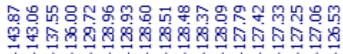<smiles>O=C1[C@H]2CC([PH+]c3ccccc3)[C@@]2(Cc2ccccc2)C(=O)N1Cc1ccccc1</smiles><smiles>O=C1[C@H](c2ccccc2)C[C@@]2(Cc3ccccc3)C[C@H](c3ccccc3)C(=O)[C@H]12</smiles>

$6: 1$ r.r.
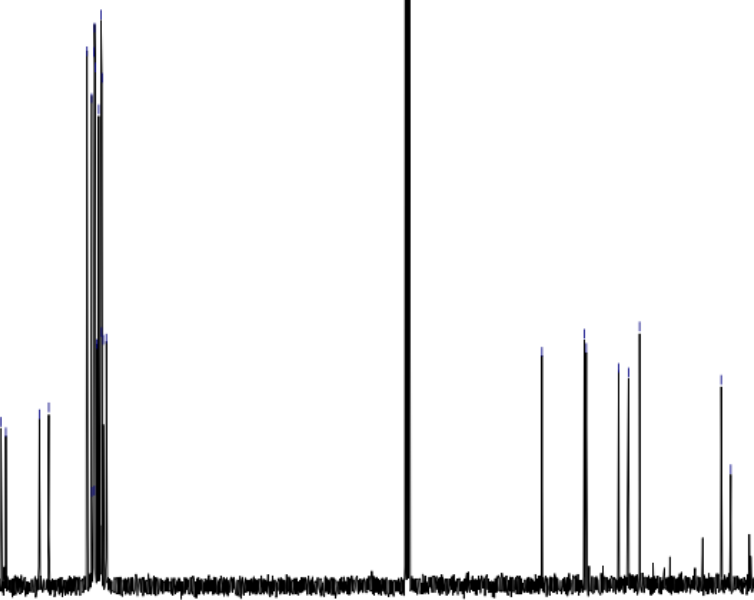

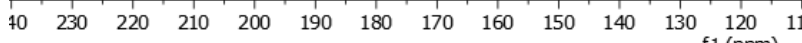


${ }^{1} \mathrm{H}$ NMR (400 MHz, $\mathrm{CDCl}_{3}$ ) of compound 39 (see procedure)

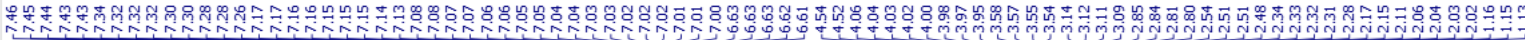
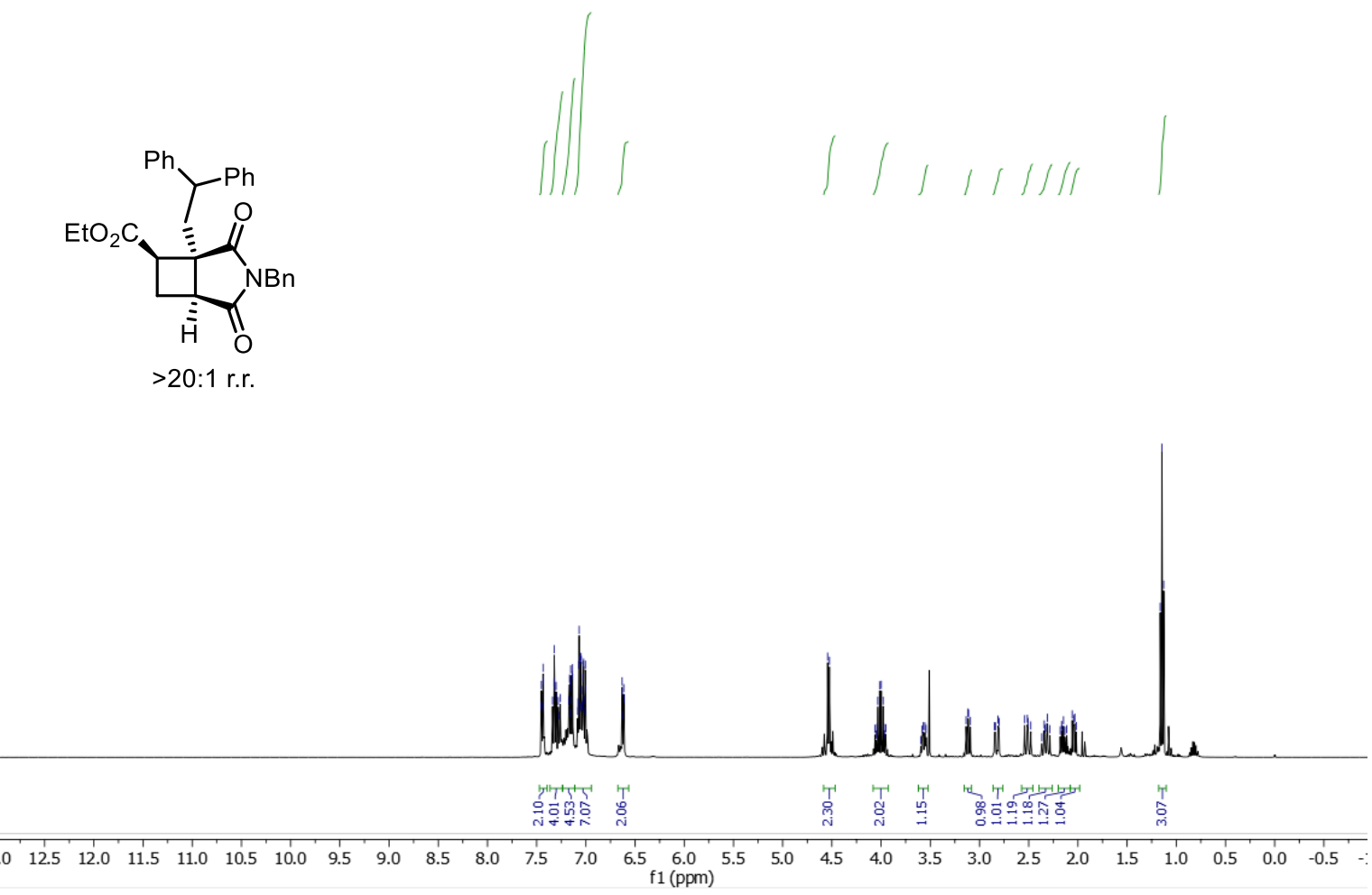

${ }^{13} \mathrm{C}$ NMR $\left(100 \mathrm{MHz}, \mathrm{CDCl}_{3}\right)$ of compound 39 (see procedure)

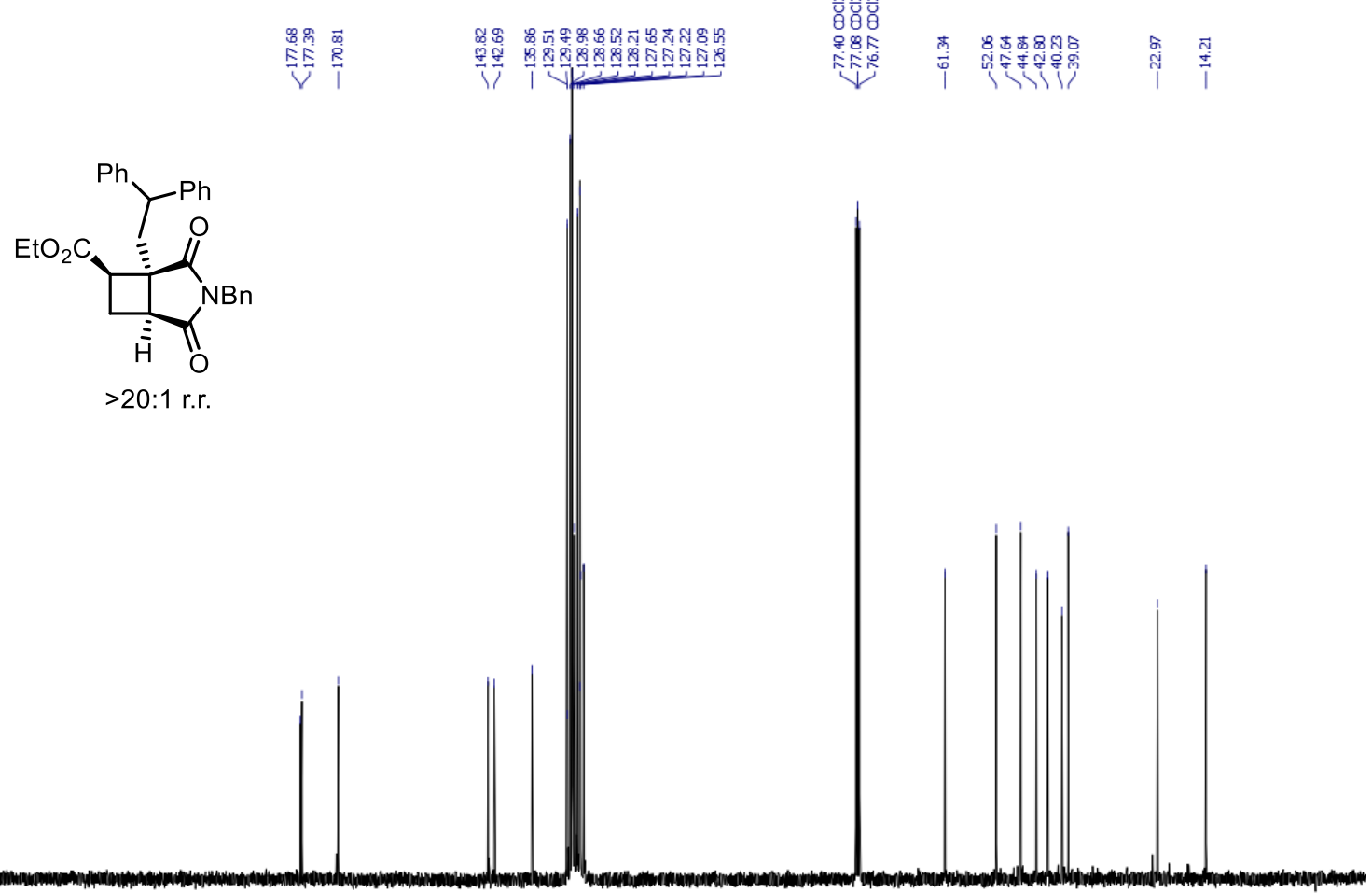

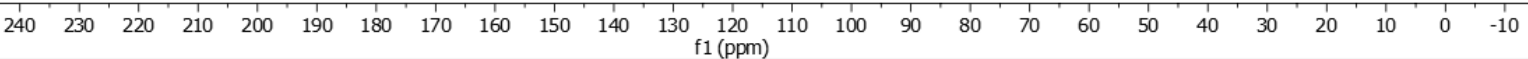


${ }^{1} \mathrm{H}$ NMR (400 MHz, $\mathrm{CDCl}_{3}$ ) of compound 40 (see procedure)

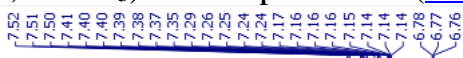
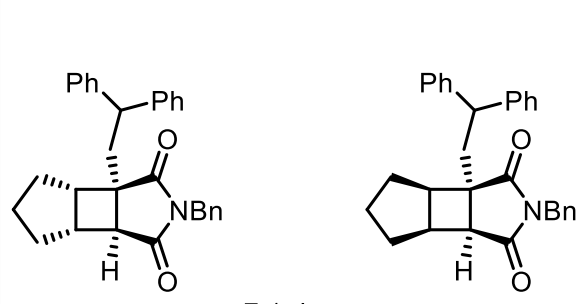

7:1 d.r.
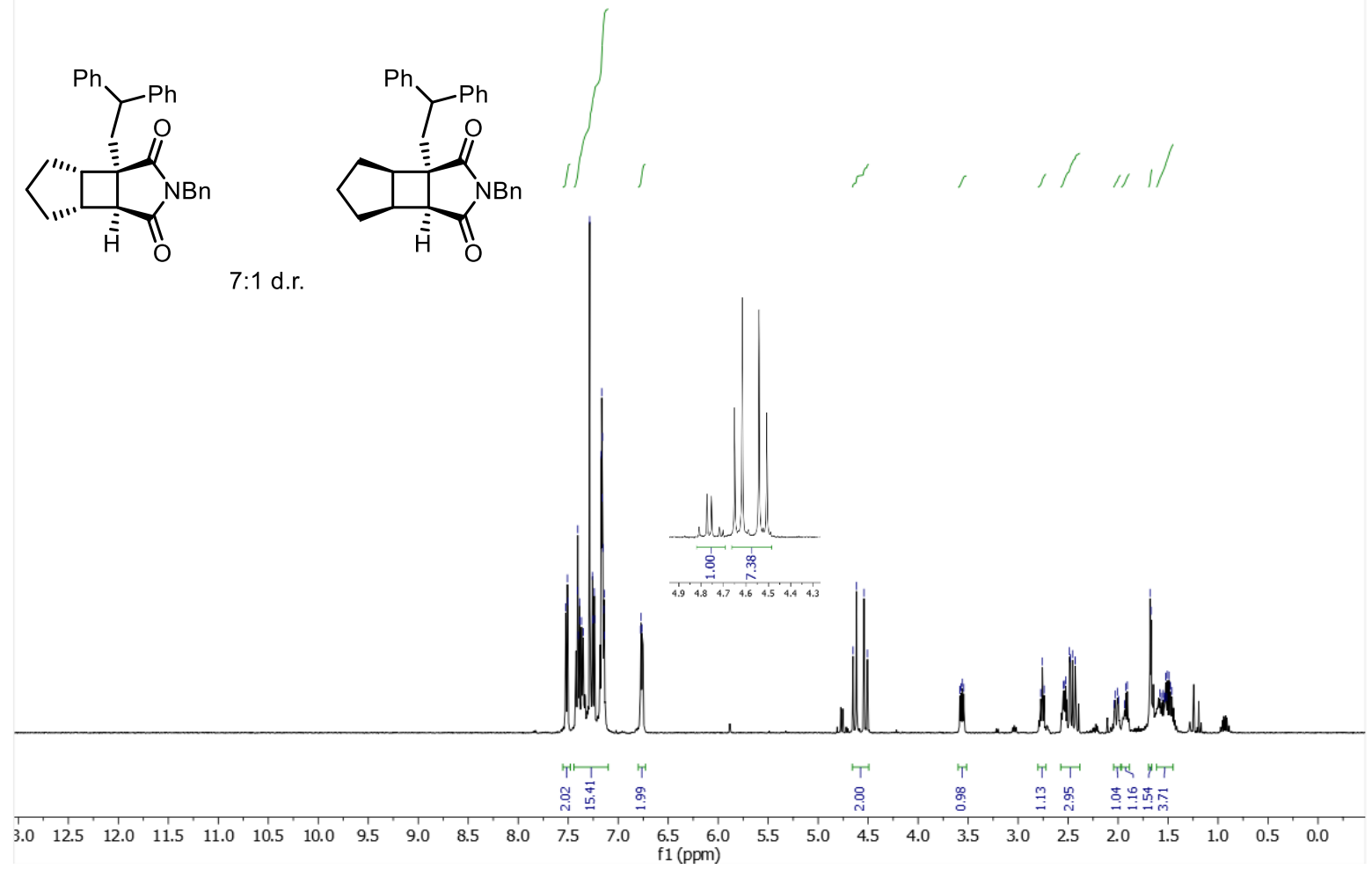

${ }^{13} \mathrm{C}$ NMR (100 MHz, $\mathrm{CDCl}_{3}$ ) of compound 40 (see procedure)

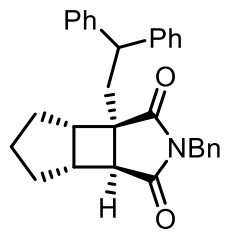

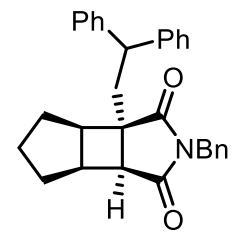

$7: 1$ d.r.

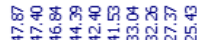

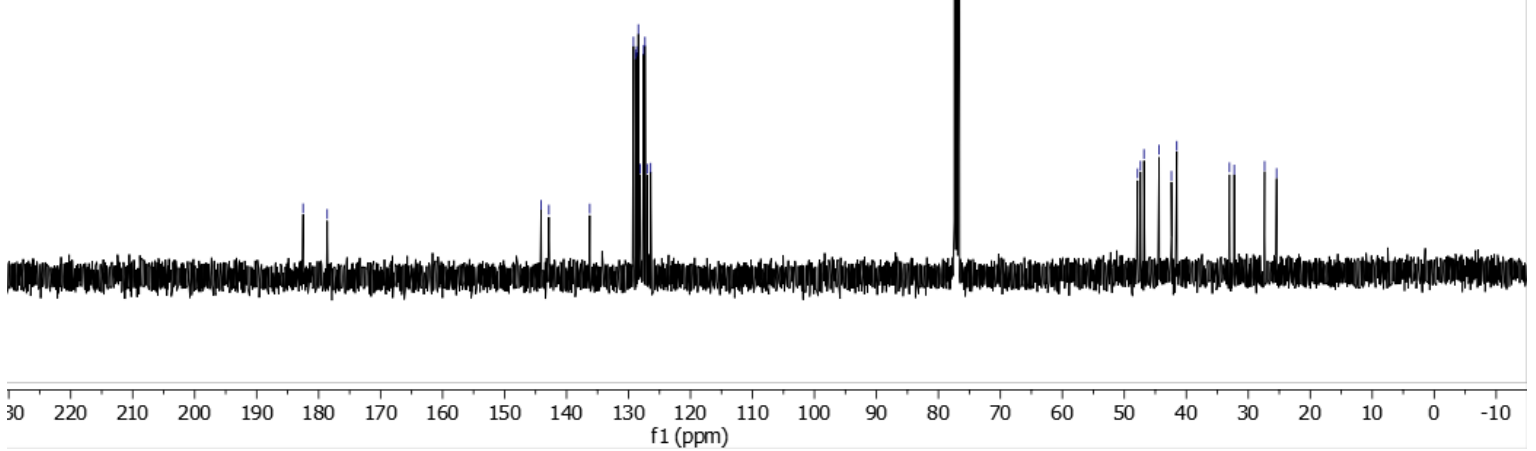


${ }^{1} \mathrm{H}$ NMR (400 MHz, $\mathrm{CDCl}_{3}$ ) of compound 41 (see procedure)

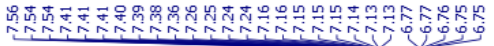

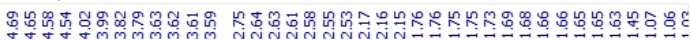<smiles></smiles>
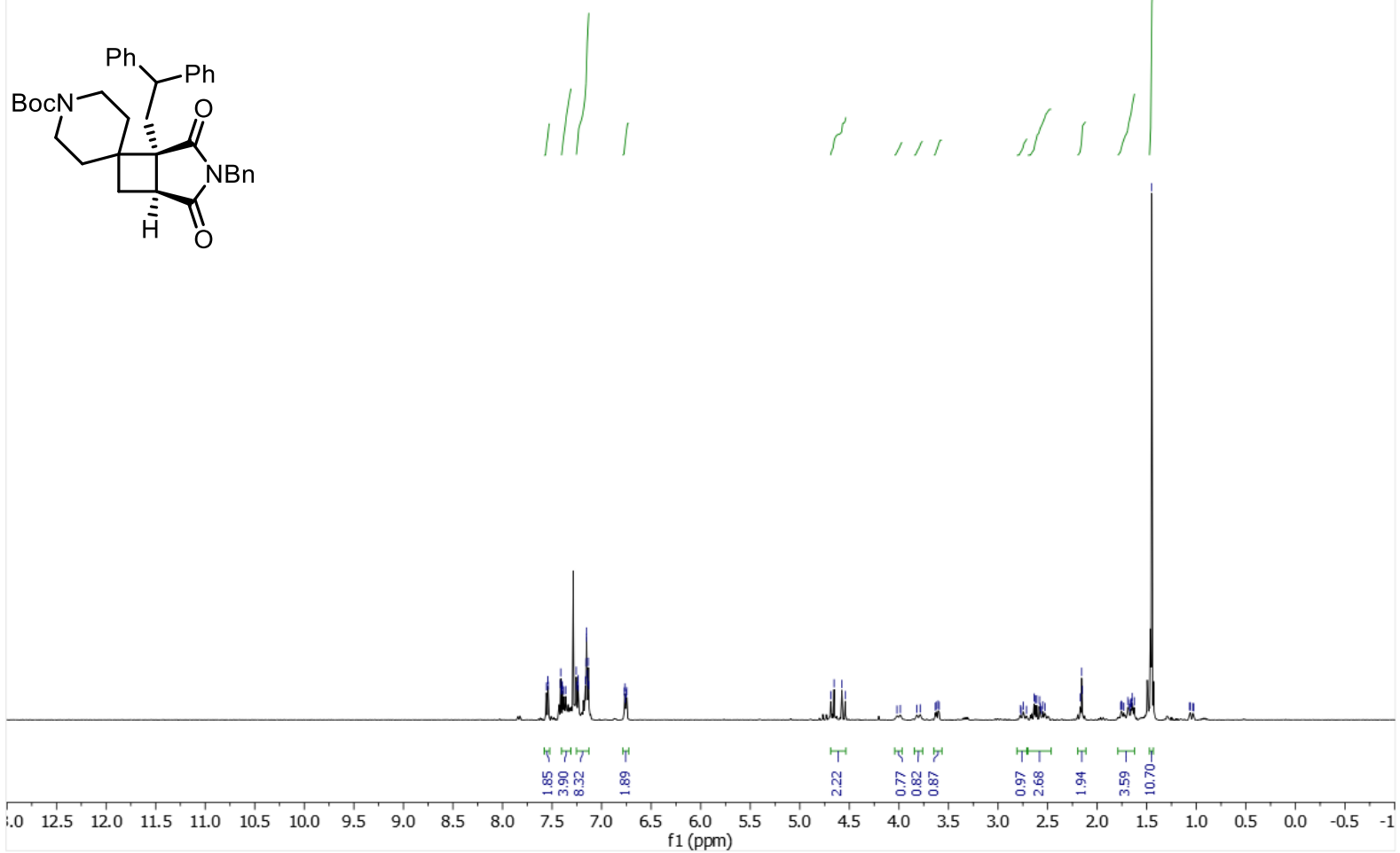

${ }^{13} \mathrm{C}$ NMR (100 MHz, $\mathrm{CDCl}_{3}$ ) of compound 41 (see procedure)

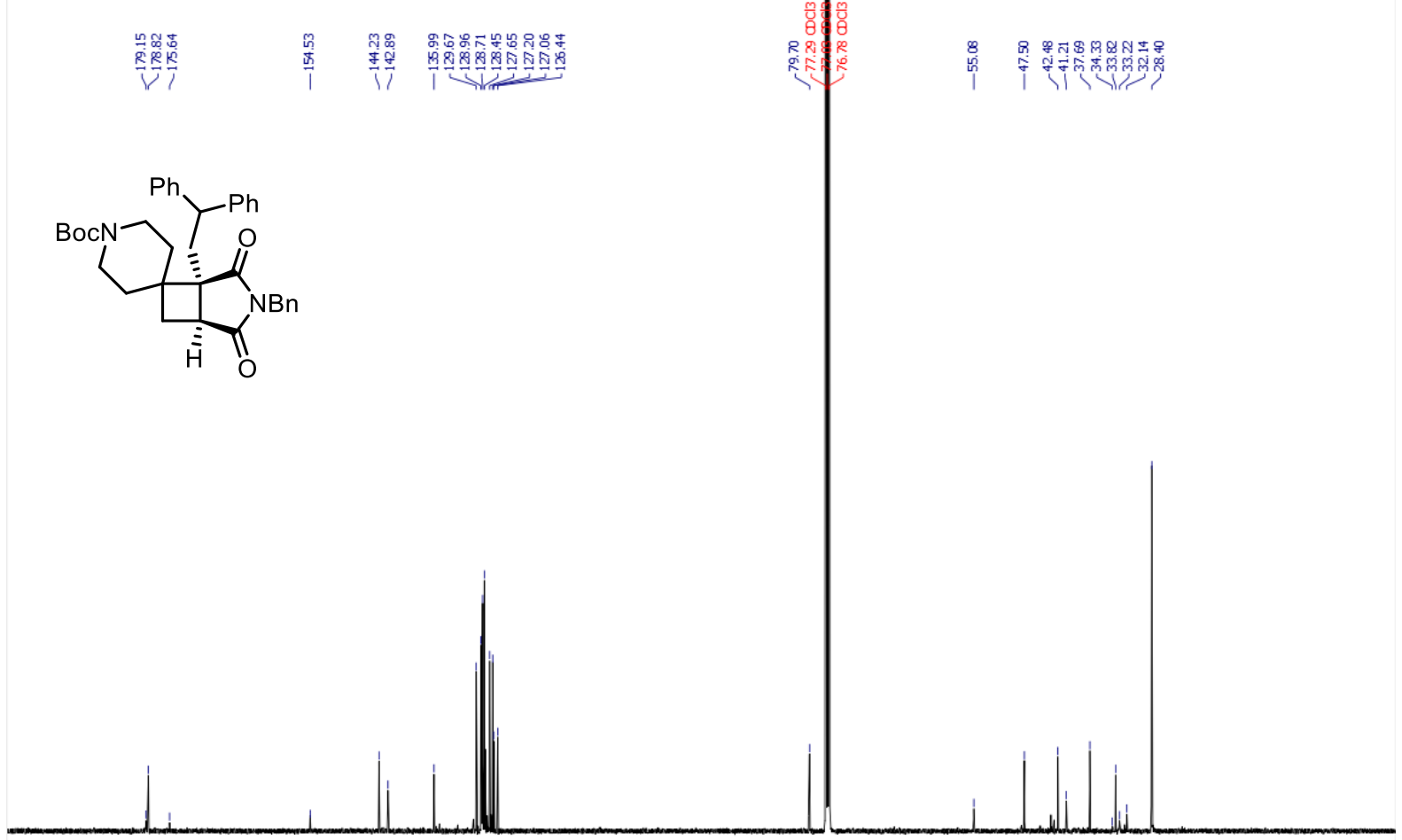

$\begin{array}{lllllllllll}190 & 180 & 170 & 160 & 150 & 140 & 130 & 120 & 110 & 100 & 90\end{array}$

$80 \quad 70 \quad 60$

50

(2)

20 
${ }^{1} \mathrm{H}$ NMR (400 MHz, $\mathrm{CDCl}_{3}$ ) of compound 42 (see procedure)

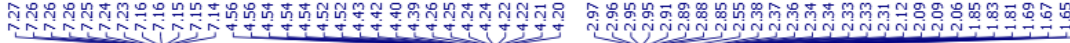<smiles>CN1NC(=O)C2C(C1=O)C1CCCOC12C(c1ccccc1)c1ccccc1</smiles>

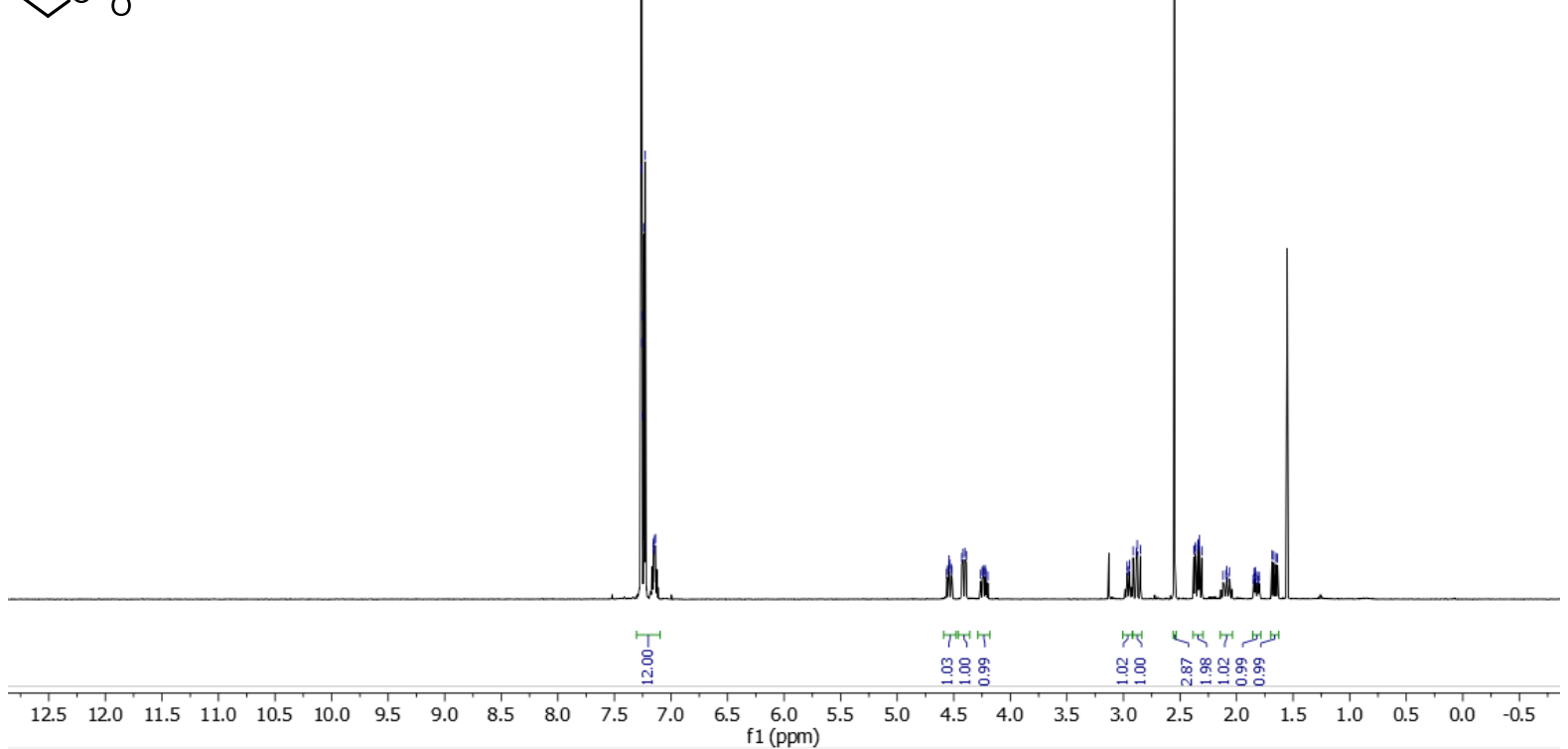

${ }^{13} \mathrm{C}$ NMR (100 MHz, $\mathrm{CDCl}_{3}$ ) of compound 42 (see procedure)

$$
\text { |c }
$$

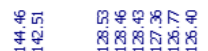

i)

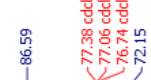

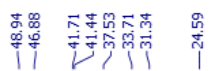
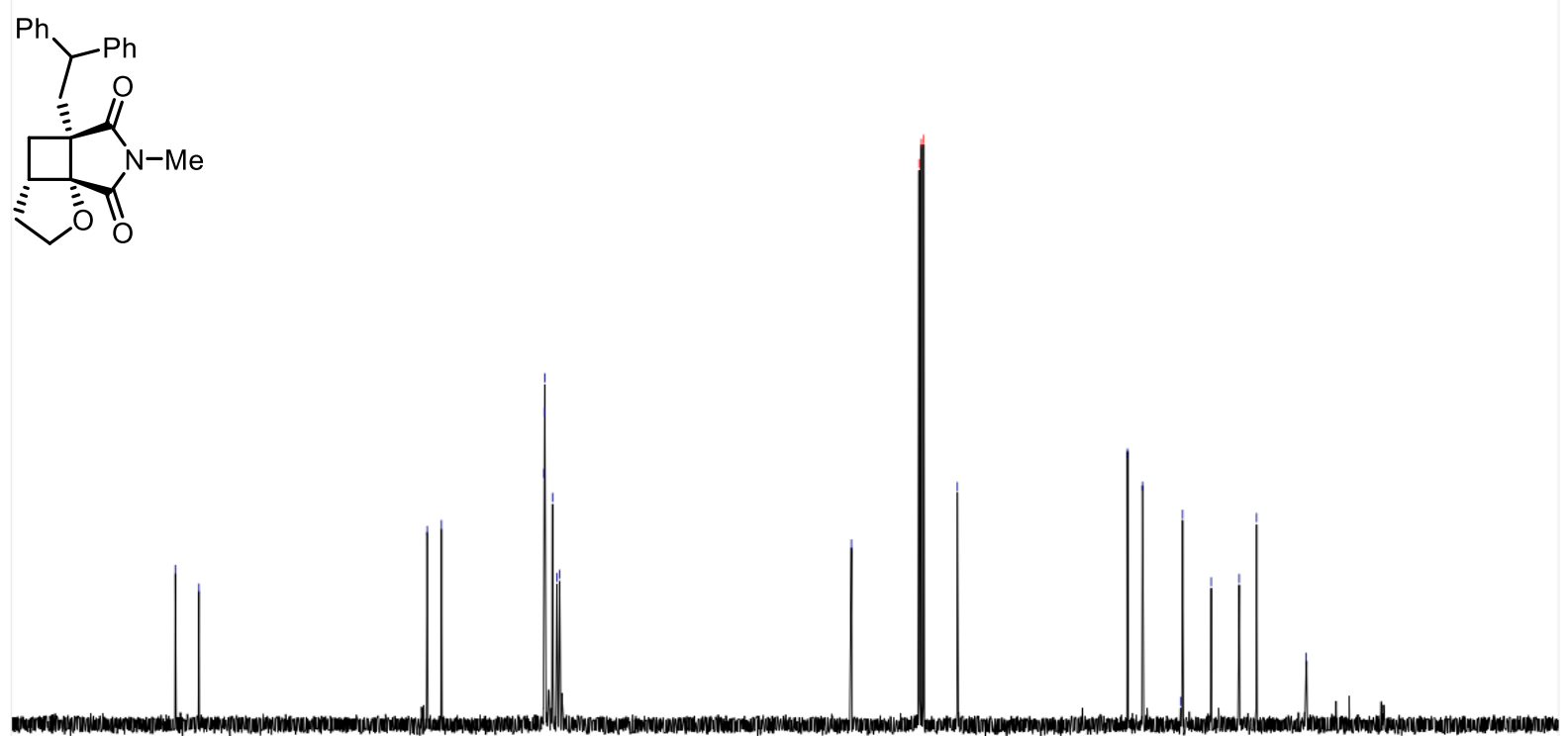

$\begin{array}{lllllllllll}100 & 190 & 180 & 170 & 160 & 150 & 140 & 130 & 120 & 110 & \begin{array}{c}100 \\ \mathrm{f} 1(\mathrm{ppm})\end{array}\end{array}$ 
${ }^{1} \mathrm{H}$ NMR (400 MHz, $\mathrm{CDCl}_{3}$ ) of compound 43 (see procedure)

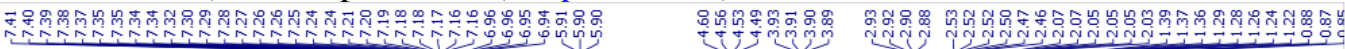<smiles>CC(C)(C)C1=C[C@H]2C(=O)N(Br)C(=O)[C@]1(CCc1ccccc1)c1ccccc12</smiles><smiles>CC(C)(C)C1C=CCC1(Cc1ccccc1)C(=O)NCc1ccccc1</smiles>

$21: 1$ r.r.
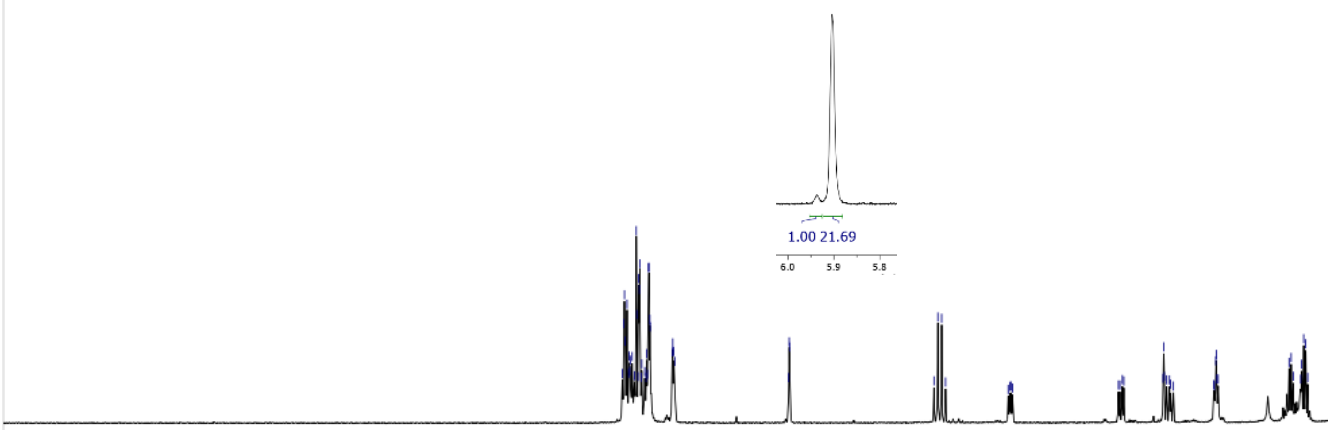

$\begin{array}{lllllllllllllllllllllllllllll}12.5 & 12.0 & 11.5 & 11.0 & 10.5 & 10.0 & 9.5 & 9.0 & 8.5 & 8.0 & 7.5 & 7.0 & 6.5 & 6.0 & 5.5 & 5.0 & 4.5 & 4.0 & 3.5 & 3.0 & 2.5 & 2.0 & 1.5 & 1.0 & 0.5 & 0.0 & -0.5\end{array}$

${ }^{13} \mathrm{C}$ NMR (100 MHz, $\left.\mathrm{CDCl}_{3}\right)$ of compound 43 (see procedure)

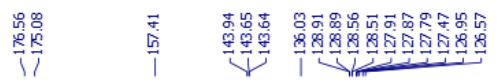

$\underbrace{\mathrm{NBn}}_{\mathrm{B}}$

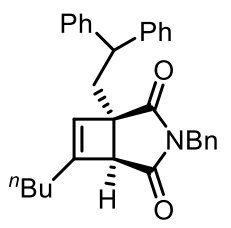

$21: 1$ r.r.

\section{응 ma}

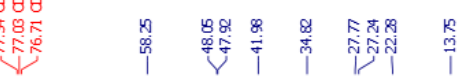

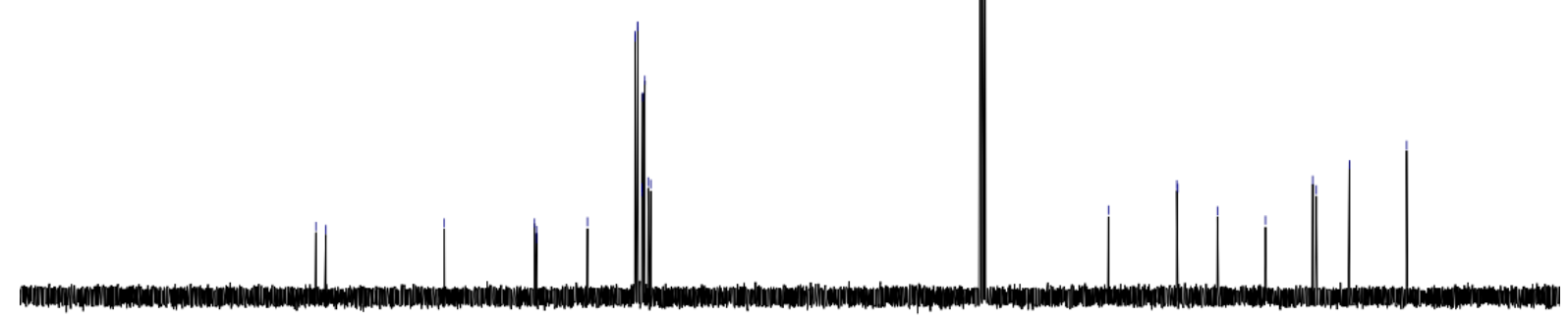

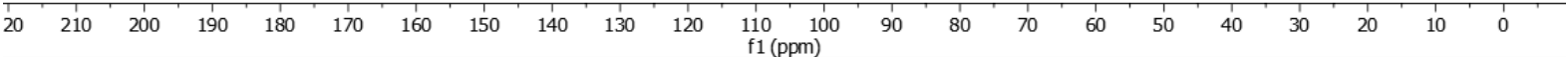


${ }^{1} \mathrm{H}$ NMR (400 MHz, $\mathrm{CDCl}_{3}$ ) of compound 44 (see procedure)

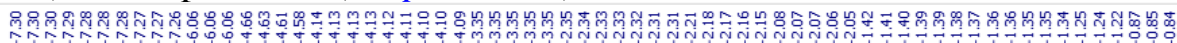
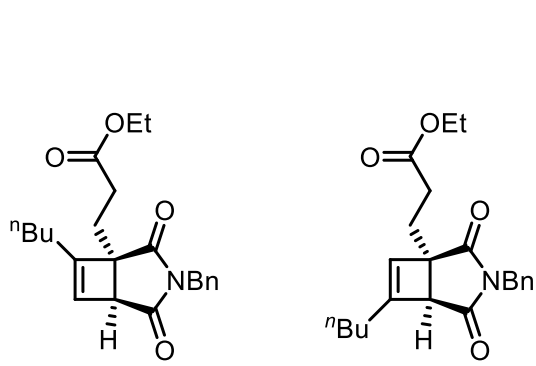

$19: 1$ r.r.
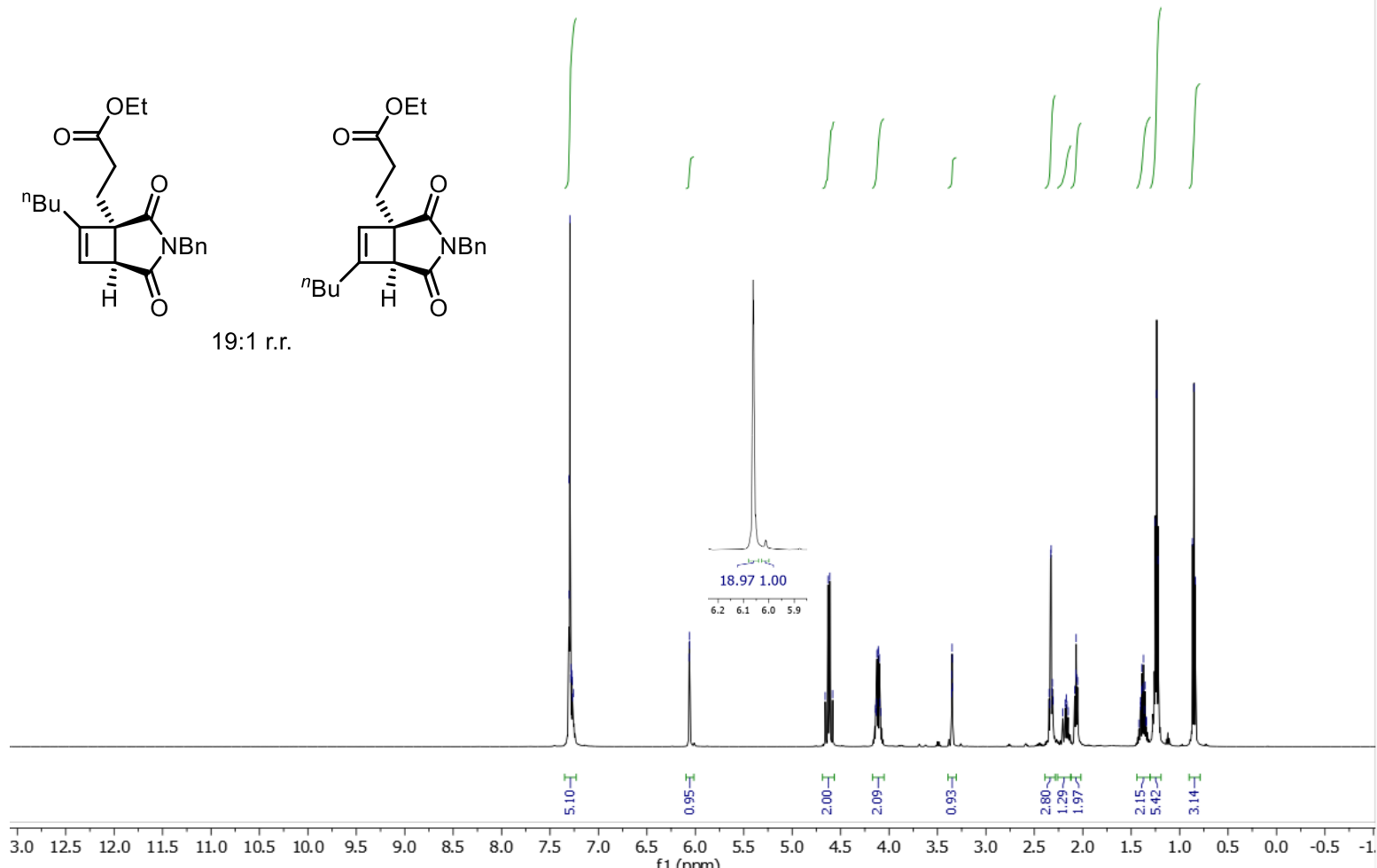

${ }^{13} \mathrm{C}$ NMR $\left(100 \mathrm{MHz}, \mathrm{CDCl}_{3}\right.$ ) of compound 44 (see procedure)
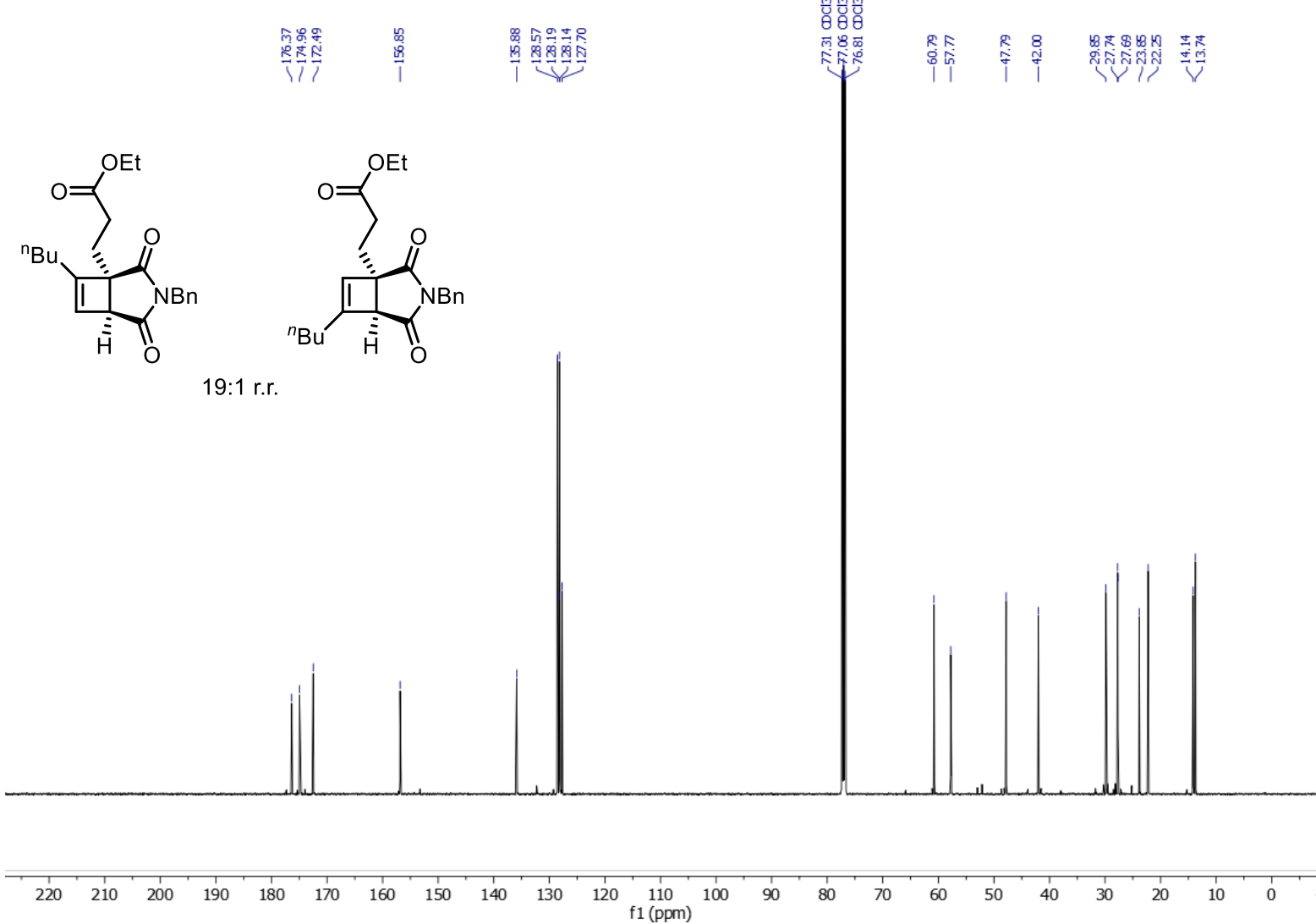
${ }^{1} \mathrm{H}$ NMR $\left(400 \mathrm{MHz}, \mathrm{CDCl}_{3}\right.$ ) of compound 52 (see procedure)

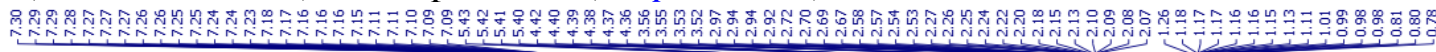<smiles>O=C(O)[C@H]1CC(Br)[C@H]1CC(c1ccccc1)c1ccccc1</smiles>
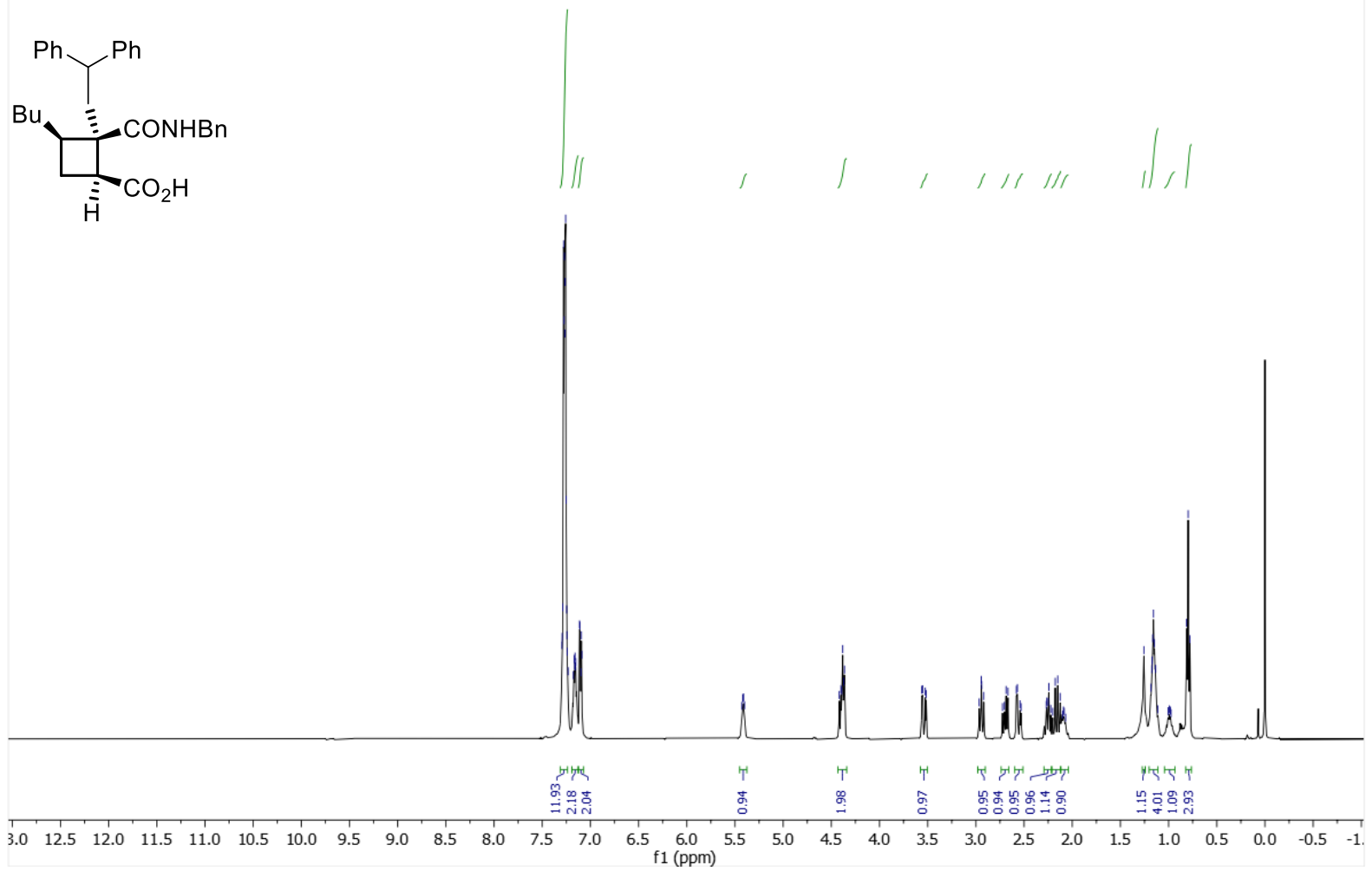

${ }^{13} \mathrm{C}$ NMR (100 MHz, DMSO- $d_{6}$ ) of compound 52 (see procedure)
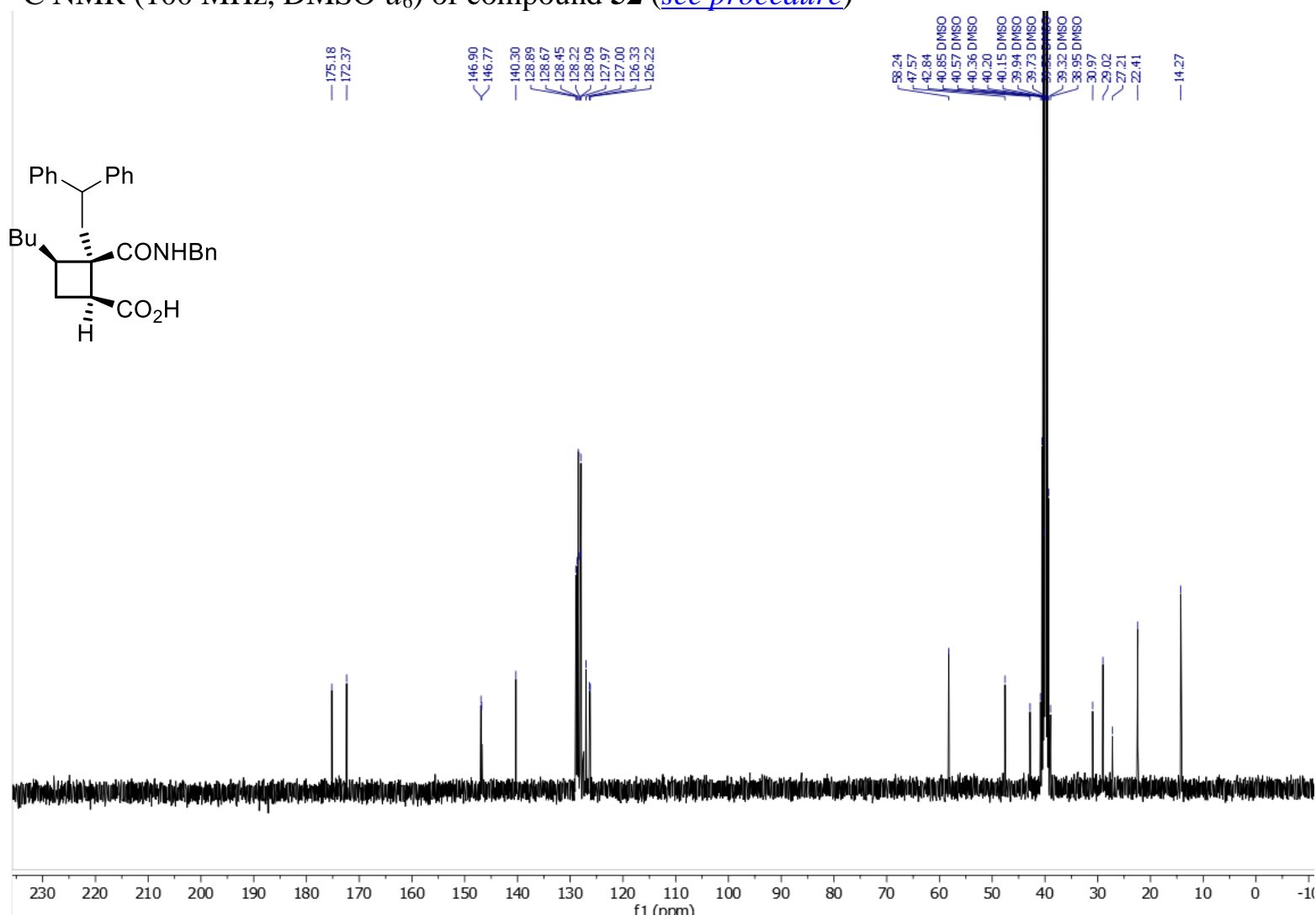
${ }^{1} \mathrm{H} \mathrm{NMR}$ (400 MHz, $\mathrm{CDCl}_{3}$ ) of compound 53 (see procedure)

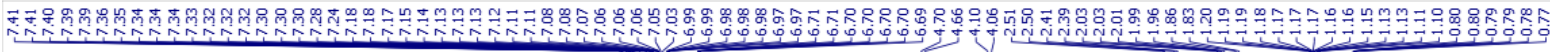

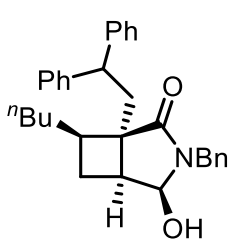

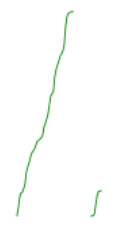

$10: 1$ d.r.
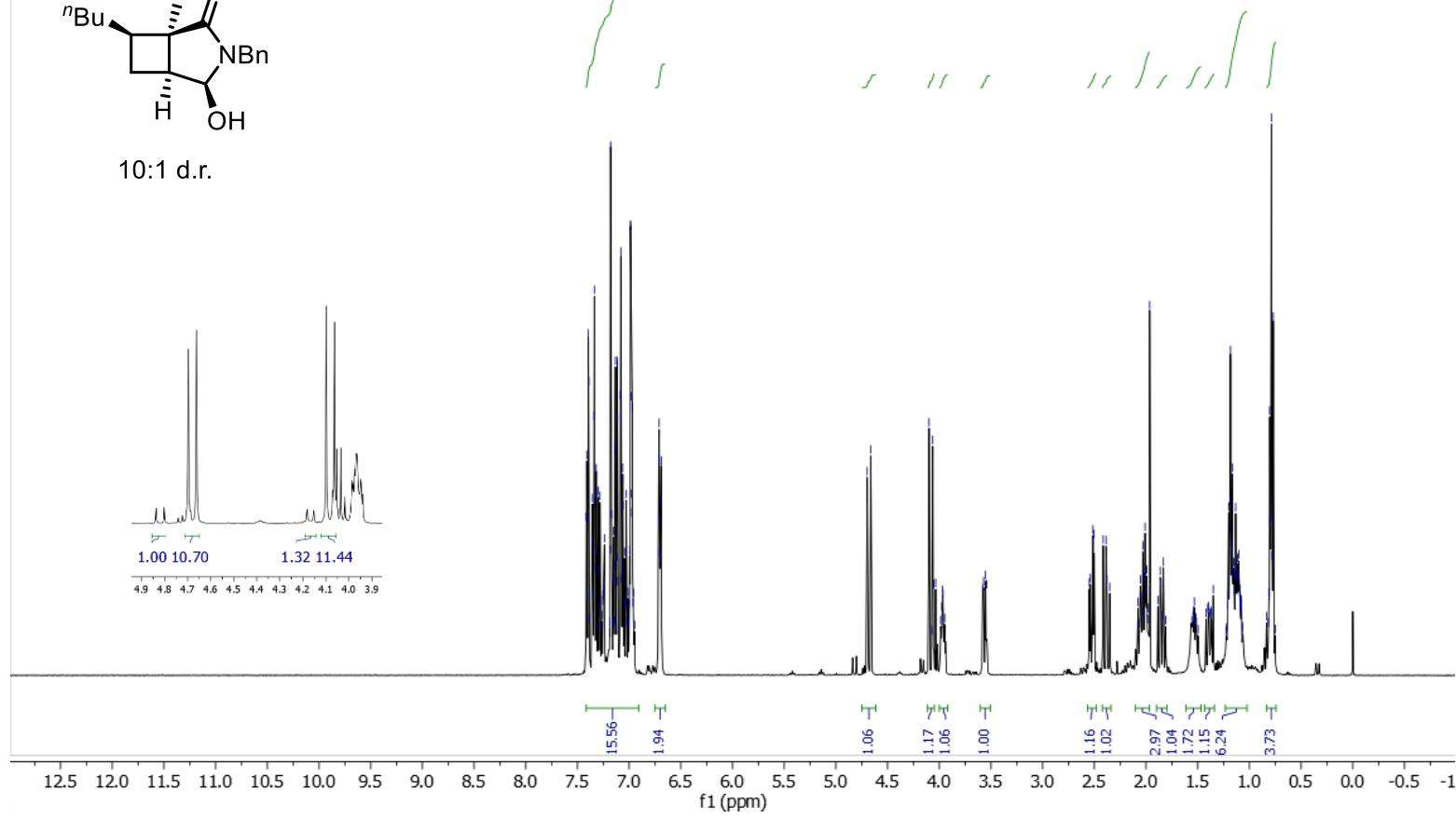

${ }^{13} \mathrm{C} \mathrm{NMR}\left(100 \mathrm{MHz}, \mathrm{CDCl}_{3}\right)$ of compound $\mathbf{5 3}$ (see procedure)

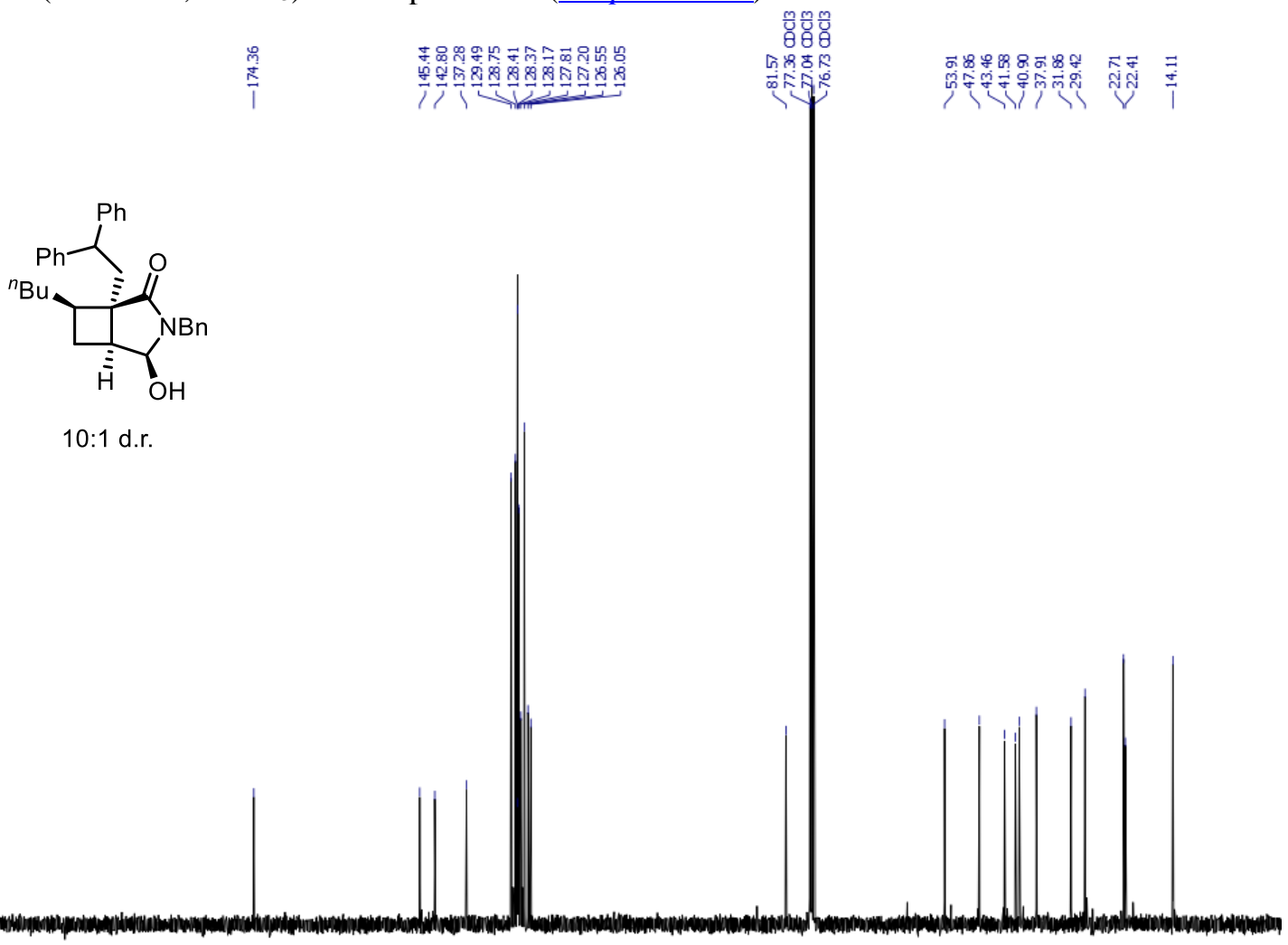

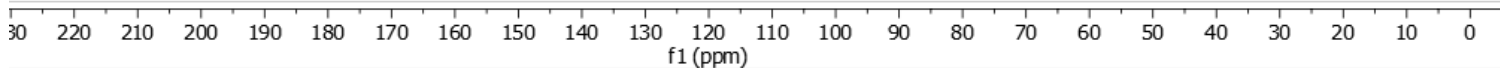


${ }^{1} \mathrm{H}$ NMR (400 MHz, $\mathrm{CDCl}_{3}$ ) of compound 54 (see procedure)

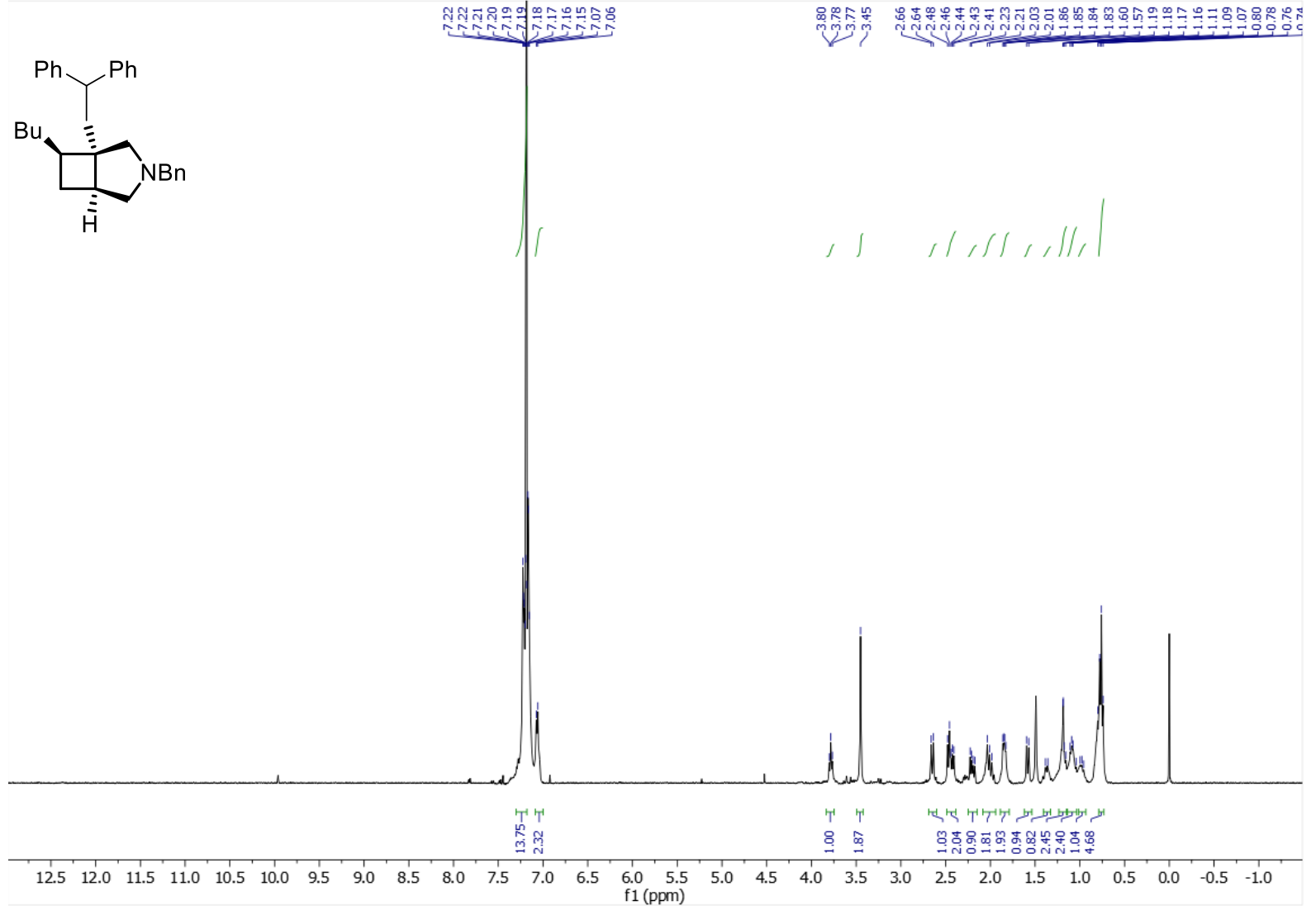

${ }^{13} \mathrm{C}$ NMR (100 MHz, $\mathrm{CDCl}_{3}$ ) of compound $\mathbf{5 4}$ (see procedure)
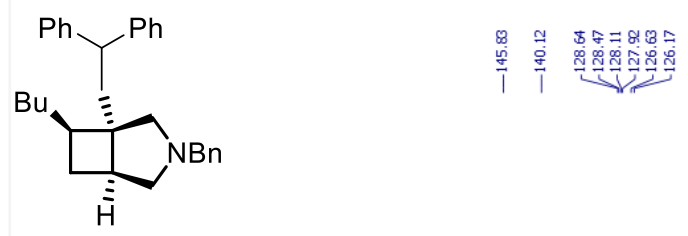

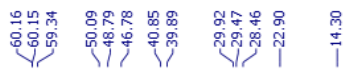

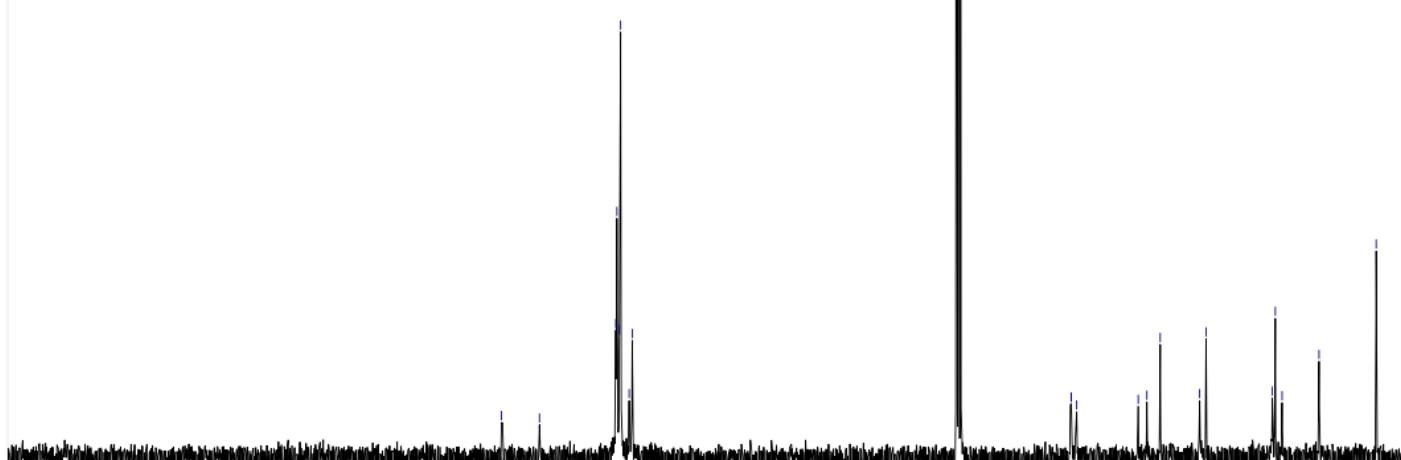

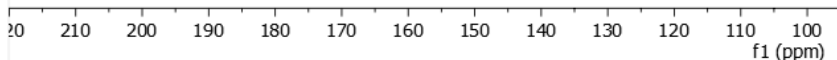


${ }^{1} \mathrm{H} \mathrm{NMR}\left(400 \mathrm{MHz}, \mathrm{CDCl}_{3}\right.$ ) of compound 55 (see procedure)

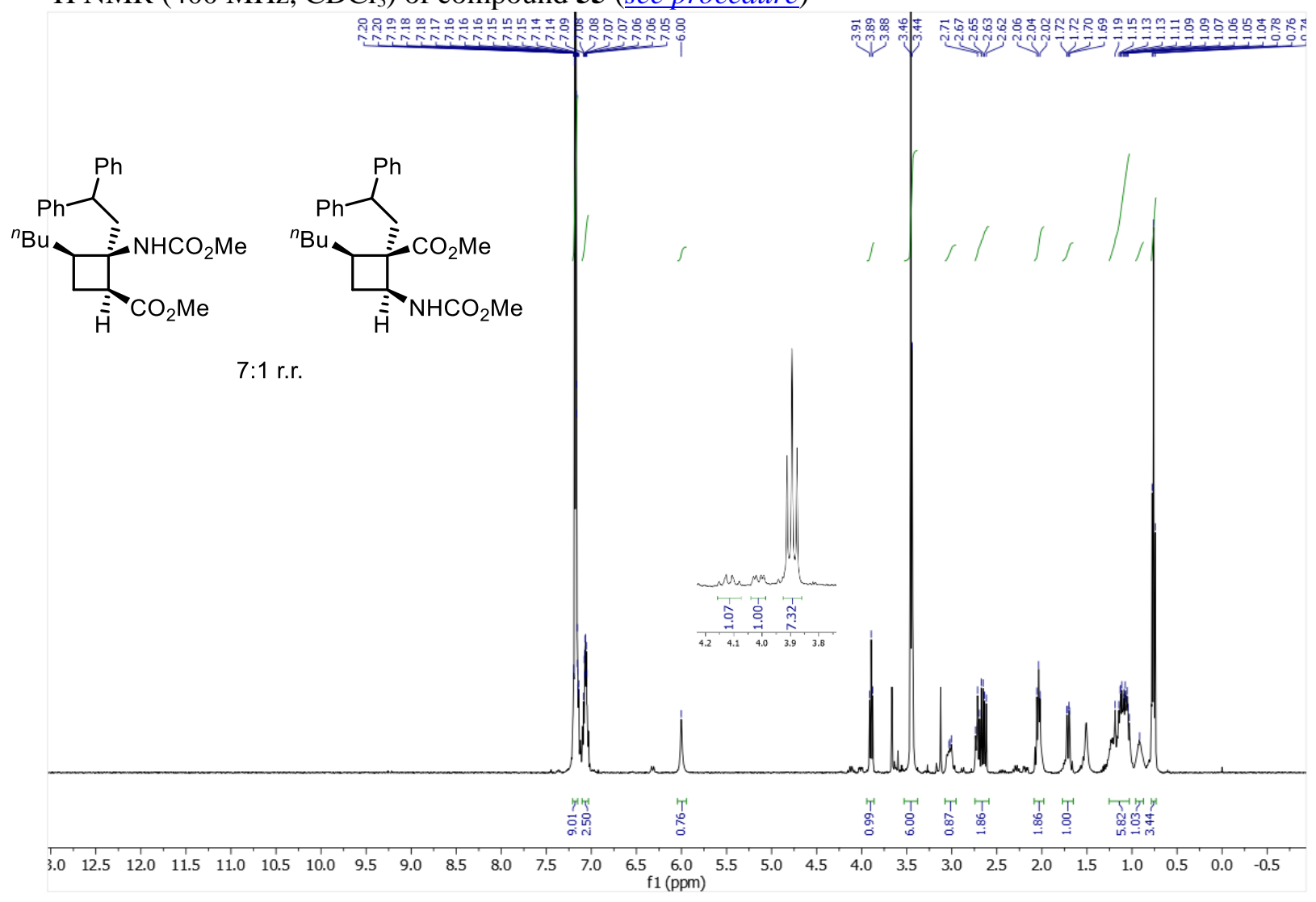

${ }^{13} \mathrm{C}$ NMR (100 MHz, $\mathrm{CDCl}_{3}$ ) of compound 55 (see procedure)

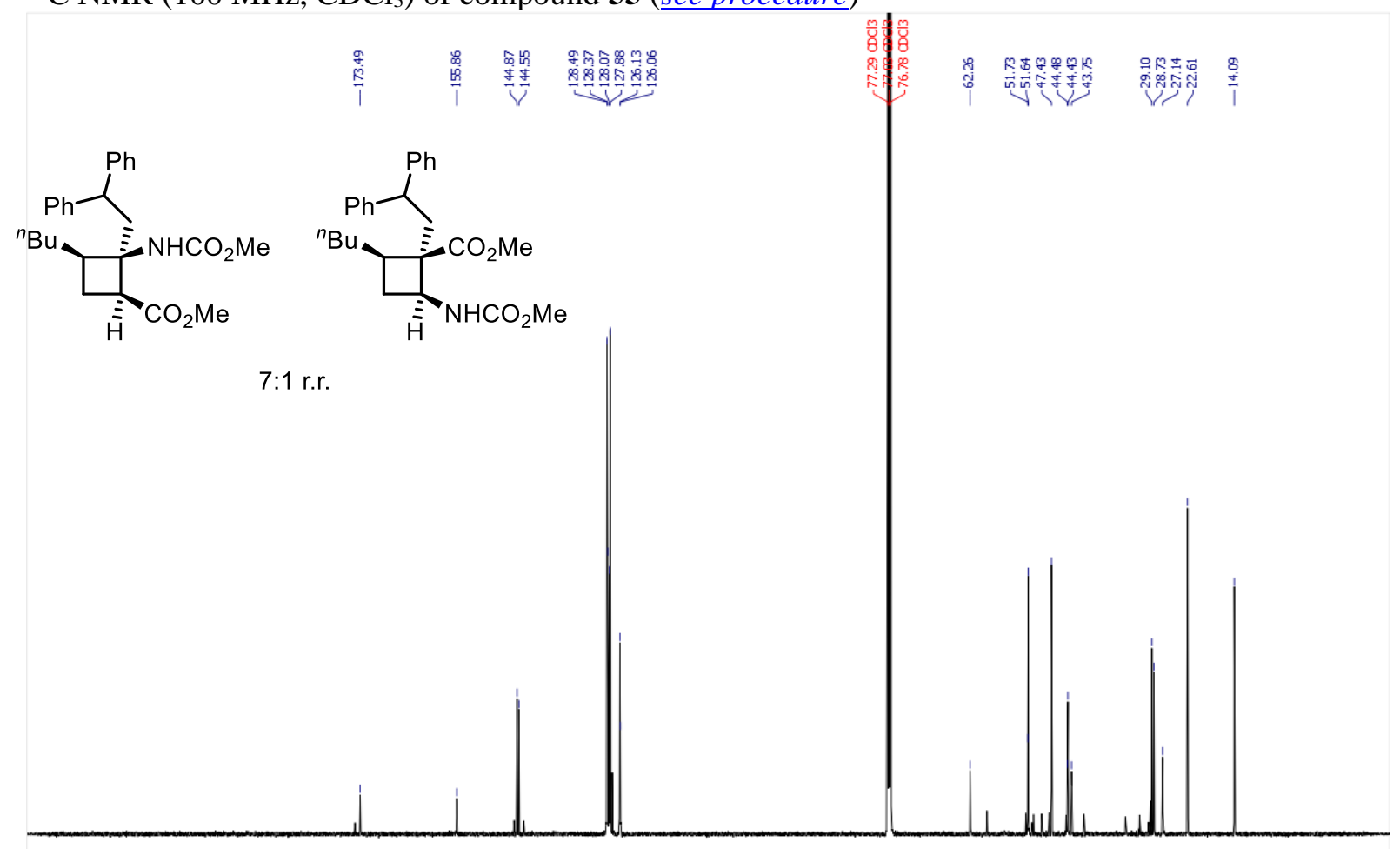

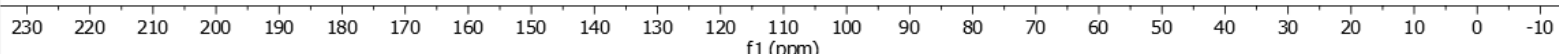

